\title{
Defying stereotypes with nanodiamonds: stable primary diamondoid phosphines
}

\section{SUPPORTING INFORMATION}

Oana Moncea, ${ }^{\mathrm{a}, \mathrm{b}, \$}$ Maria A. Gunawan, ${ }^{\mathrm{a}, \mathrm{b}, \$}$ Didier Poinsot, ${ }^{\mathrm{a}}$ Hélène Cattey, ${ }^{\mathrm{a}}$ Jonathan Becker, ${ }^{\mathrm{c}}$ Raisa I. Yurchenko, ${ }^{\mathrm{d}}$ Ekaterina D. Butova, ${ }^{\mathrm{d}}$ Heike Hausmann, ${ }^{\mathrm{b}}$ Marina Šekutor, ${ }^{\mathrm{b}}$ Andrey A. Fokin, ${ }^{\text {b,d }}$ Jean-Cyrille Hierso*a,e and Peter R. Schreiner* ${ }^{* \mathrm{~b}}$

${ }^{a}$ Institut de Chimie Moléculaire de l'Université de Bourgogne (ICMUB), UMR-CNRS 6302, Université de Bourgogne Franche-Comté, 9 avenue Alain Savary, 21078 Dijon, France

${ }^{b}$ Institut für Organische Chemie, Justus-Liebig-Universität, Heinrich-Buff-Ring 17, 35392 Giessen, Germany,prs@org.chemie.uni-giessen.de

${ }^{c}$ Institut für Anorganische und Analytische Chemie, Justus-Liebig-Universität, Heinrich-BuffRing 17, 35392 Giessen, Germany

${ }^{d}$ Department of Organic Chemistry, Kiev Polytechnic Institute, Pr. Pobedy 37, 03056 Kiev, Ukraine, aaf@xtf.kpi.ua

${ }^{e}$ Institut Universitaire de France (IUF), 103 Bd. Saint Michel, 75005 Paris Cedex 5, France, jean-cyrille.hierso@u-bourgogne.fr

${ }^{\$}$ These authors contributed equally 


\section{Contents:}

1) NMR spectra $\quad$ S3 $-\mathrm{S100}$

2) VT-NMR studies for primary phosphines 31 in different solvents $\quad$ S101 - S105

3) Coordinates of diamondoid radical cations $\quad$ S106 - S110

4) Stationary structures for secondary diamondoid phosphines $\quad$ S111 - S122

$\begin{array}{lr}\text { 5) X-Ray structures } & \text { S123 - S196 }\end{array}$ 


\section{NMR spectra}

1.1. 4,9-Dichlorodiamantane (11)-prepared from 4,9-dihydroxydiamantane (10)

1.1.1. $\quad 4,9-\mathrm{Dia}-\mathrm{Cl}_{2}{ }^{1} \mathrm{H}$

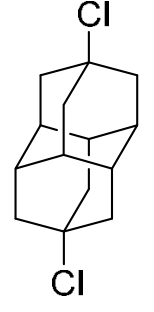

11
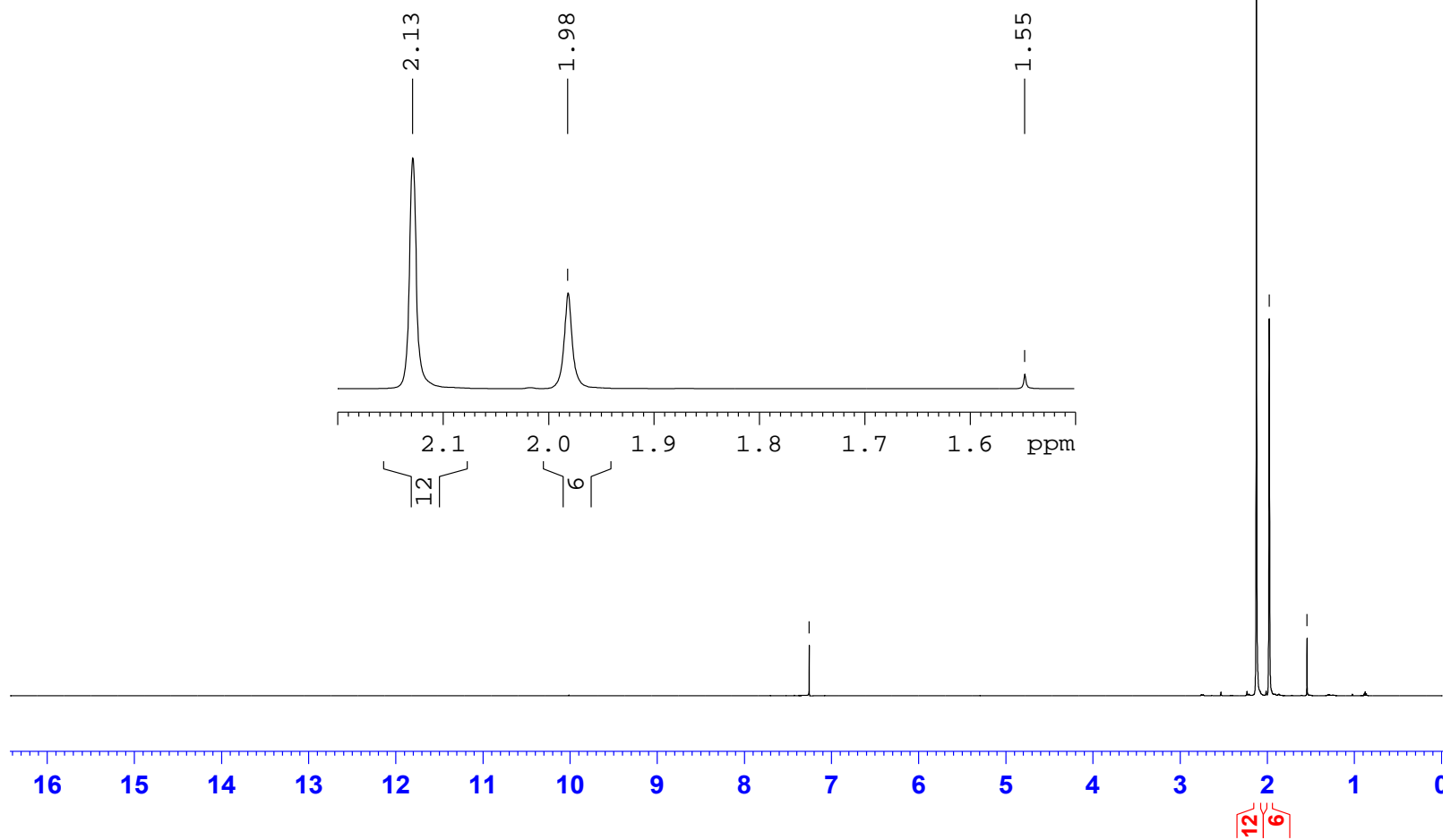
1.1.2. $4,9-\mathrm{Dia}-\mathrm{Cl}_{2}{ }^{13} \mathrm{C}$

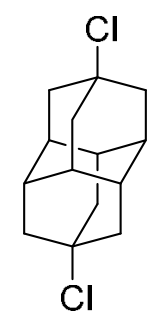

11

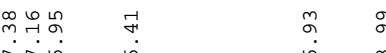

Y $\mid 1$

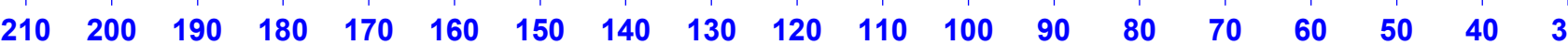


1.2. (9-Chlorodiamant-4-yl)phosphonic dichloride (12)-prepared from 4,9-dihydroxydiamantane (10)

1.2.1. 9-Cl-dia-4- $\mathrm{POCl}_{2}{ }^{1} \mathrm{H}$

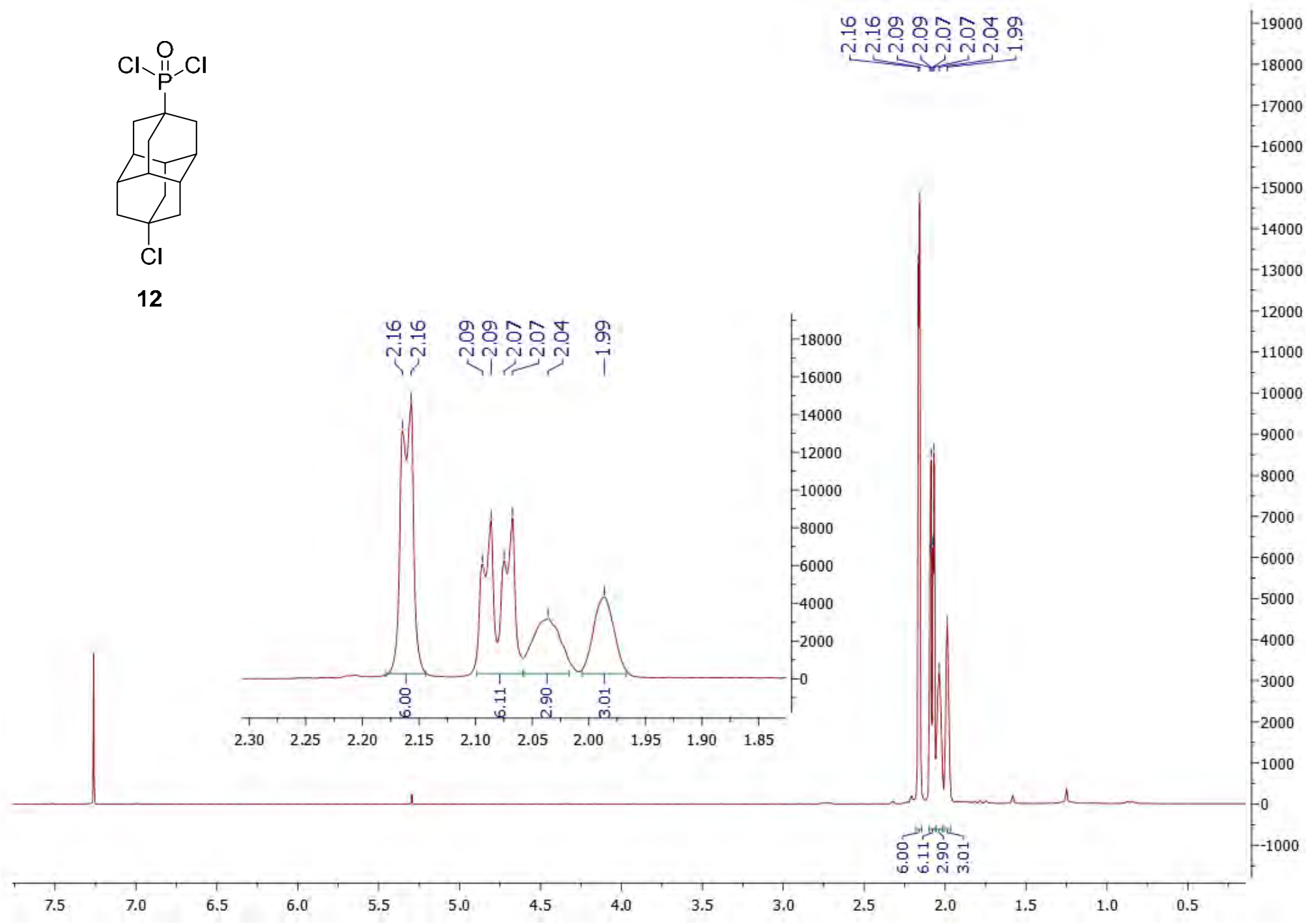


1.2.2. 9-Cl-dia-4- $\mathrm{POCl}_{2}{ }^{13} \mathrm{C}$

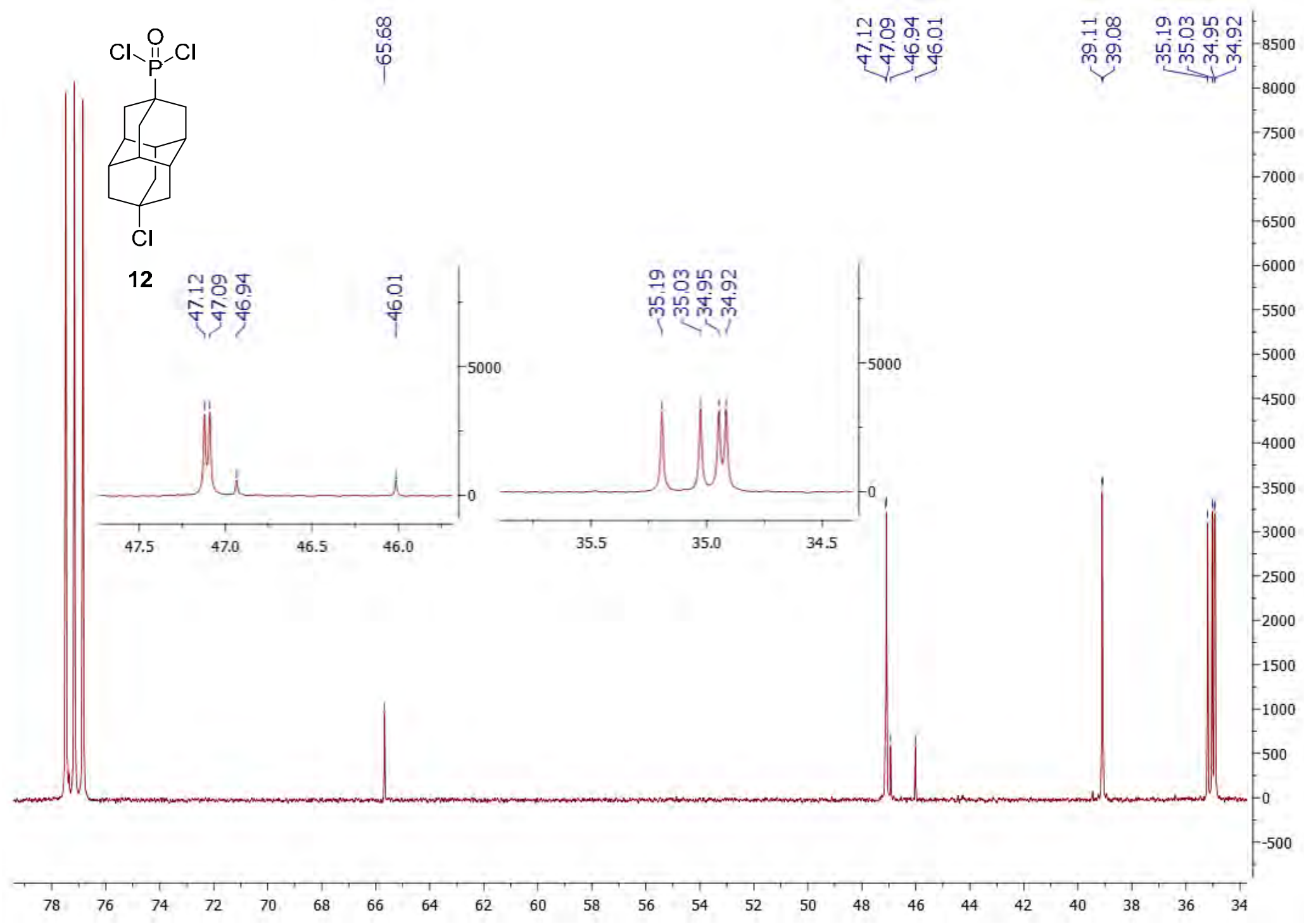


1.2.3. 9-Cl-dia-4- $\mathrm{POCl}_{2}{ }^{31} \mathrm{P}$

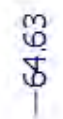

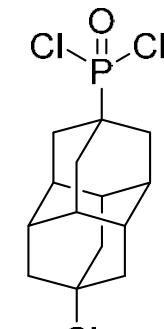

12

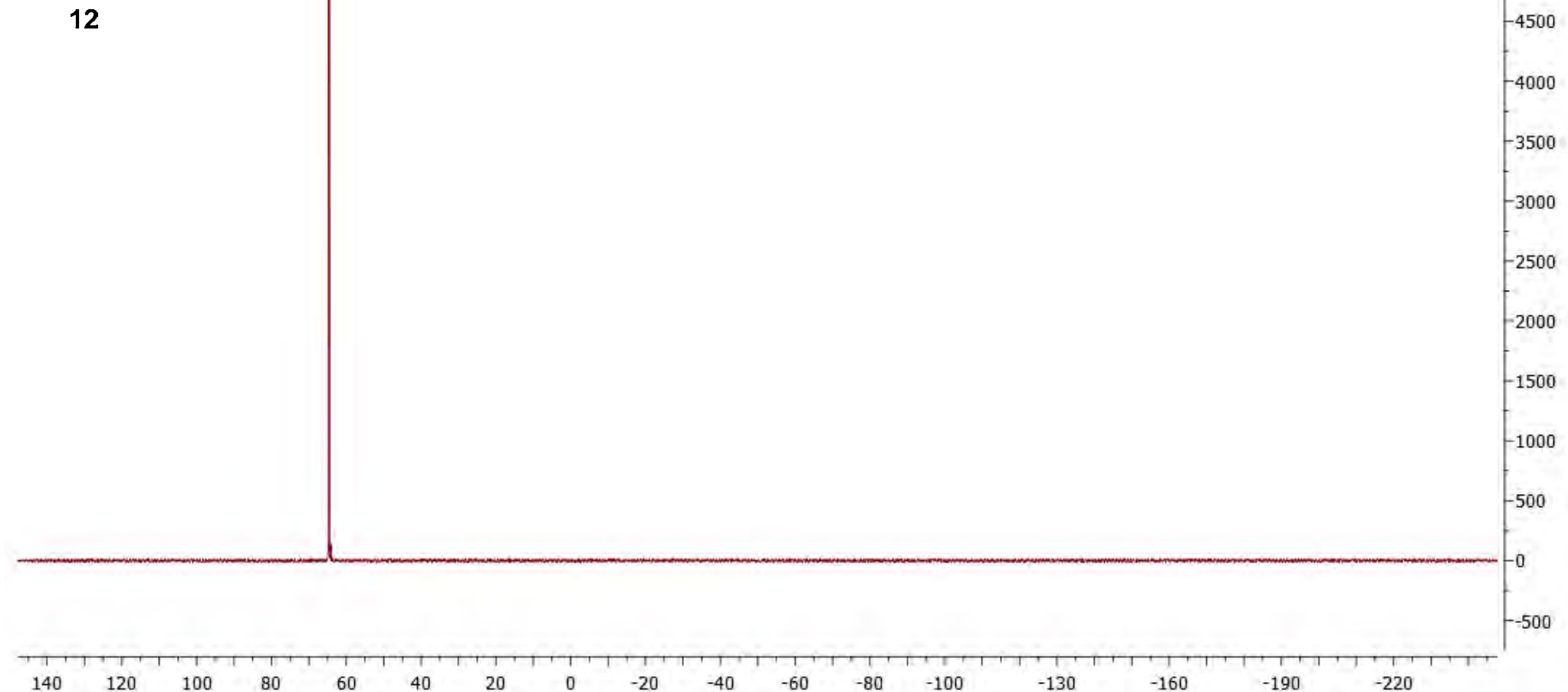


1.3. (4,9-diamantyl)diphosphonic dichloride (13)-prepared from 4,9-dihydroxydiamantane (10)

1.3.1. $\quad 4,9$-dia- $\left(\mathrm{POCl}_{2}\right)_{2}{ }^{1} \mathrm{H}$

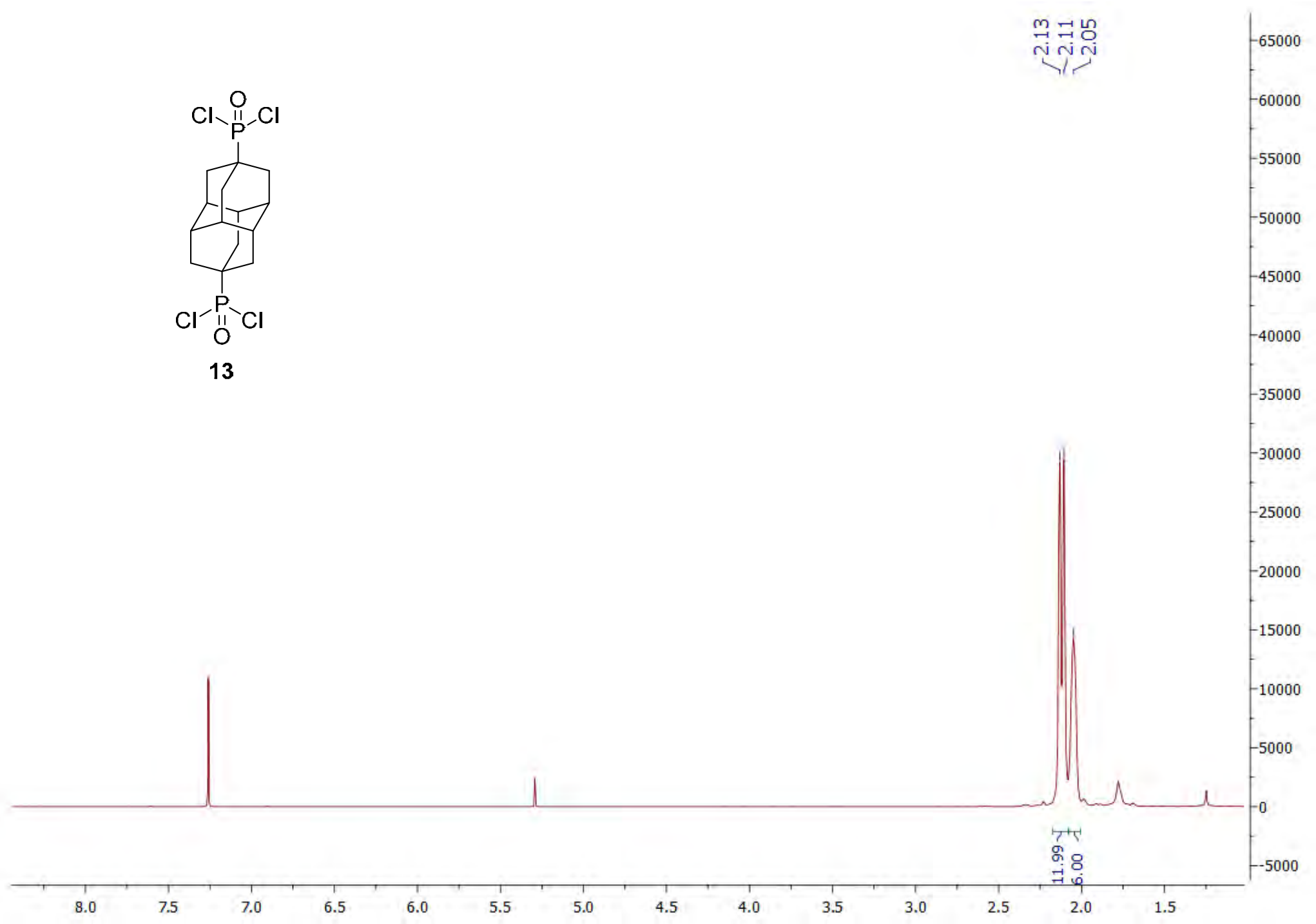


1.3.2. $4,9-$ dia- $\left(\mathrm{POCl}_{2}\right)_{2}{ }^{13} \mathrm{C}$

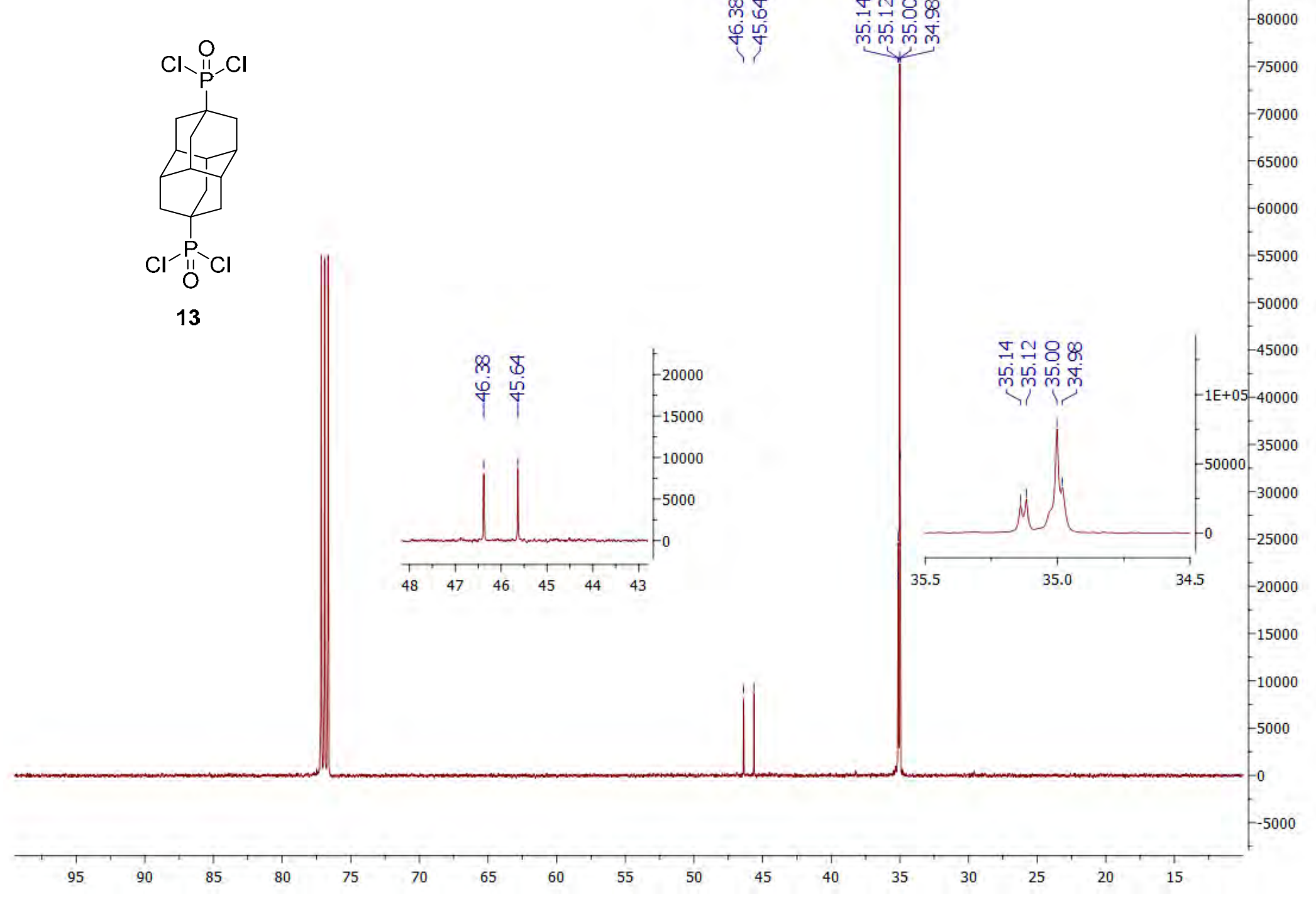


1.3.3. $4,9-$ dia- $\left(\mathrm{POCl}_{2}\right)_{2}{ }^{31} \mathrm{P}$

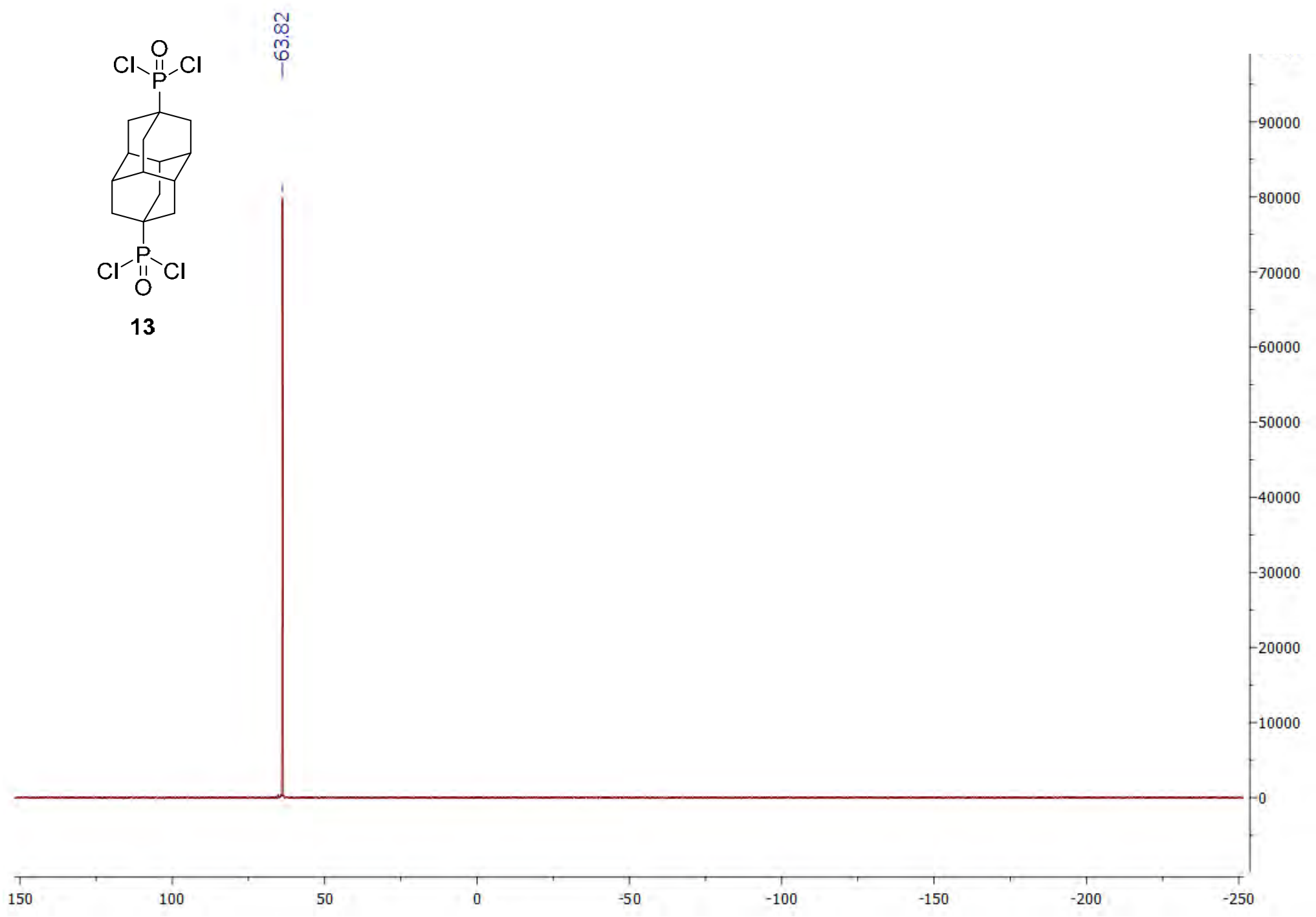


1.4. (9-Hydroxydiamant-4-yl)phosphonic dichloride (14)-prepared from 4,9-dihydroxydiamantane (10)

1.4.1. 9-OH-dia-4- $\mathrm{POCl}_{2}{ }^{1} \mathrm{H}$

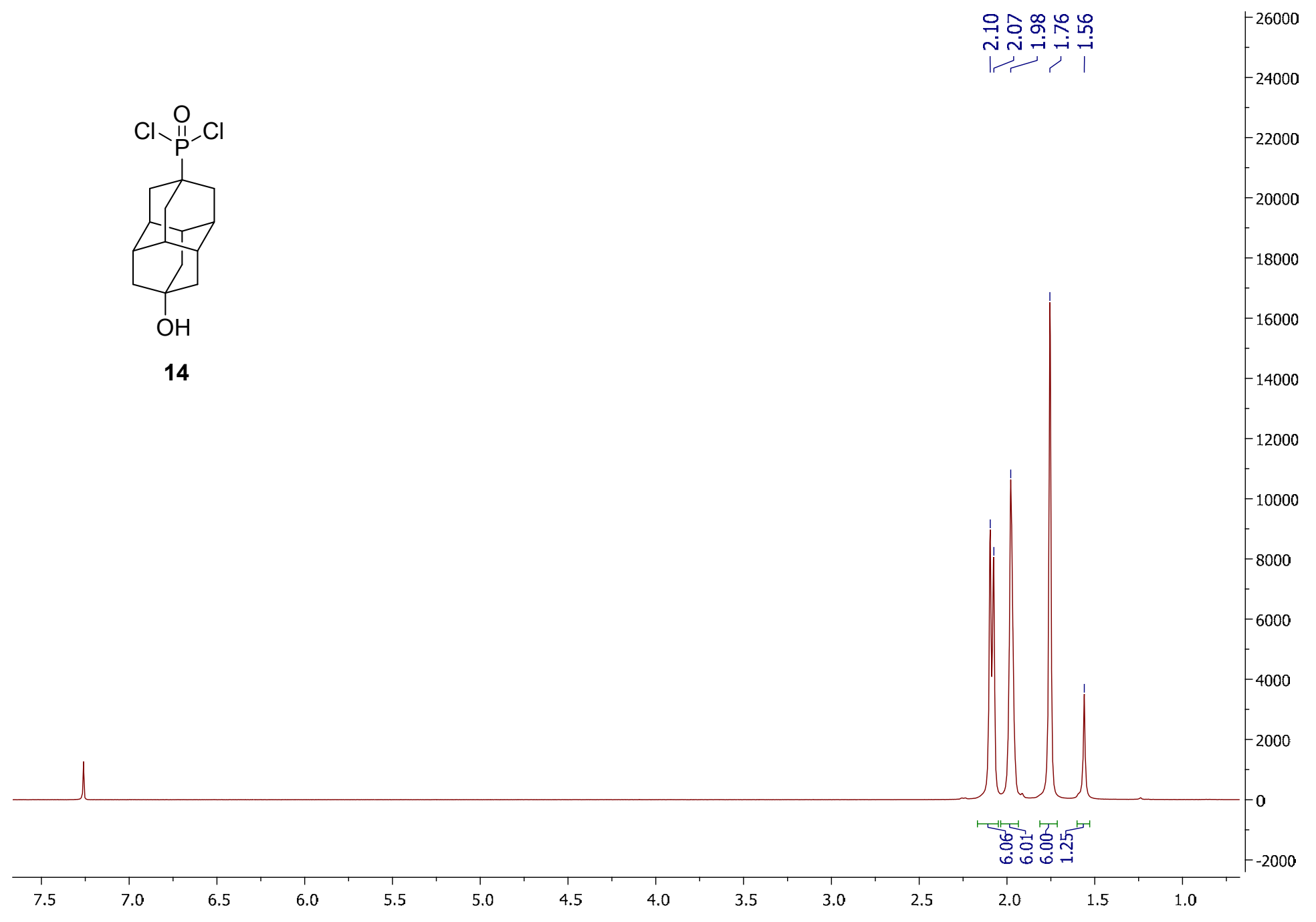


1.4.2. 9-OH-dia-4- $\mathrm{POCl}_{2}{ }^{13} \mathrm{C}$

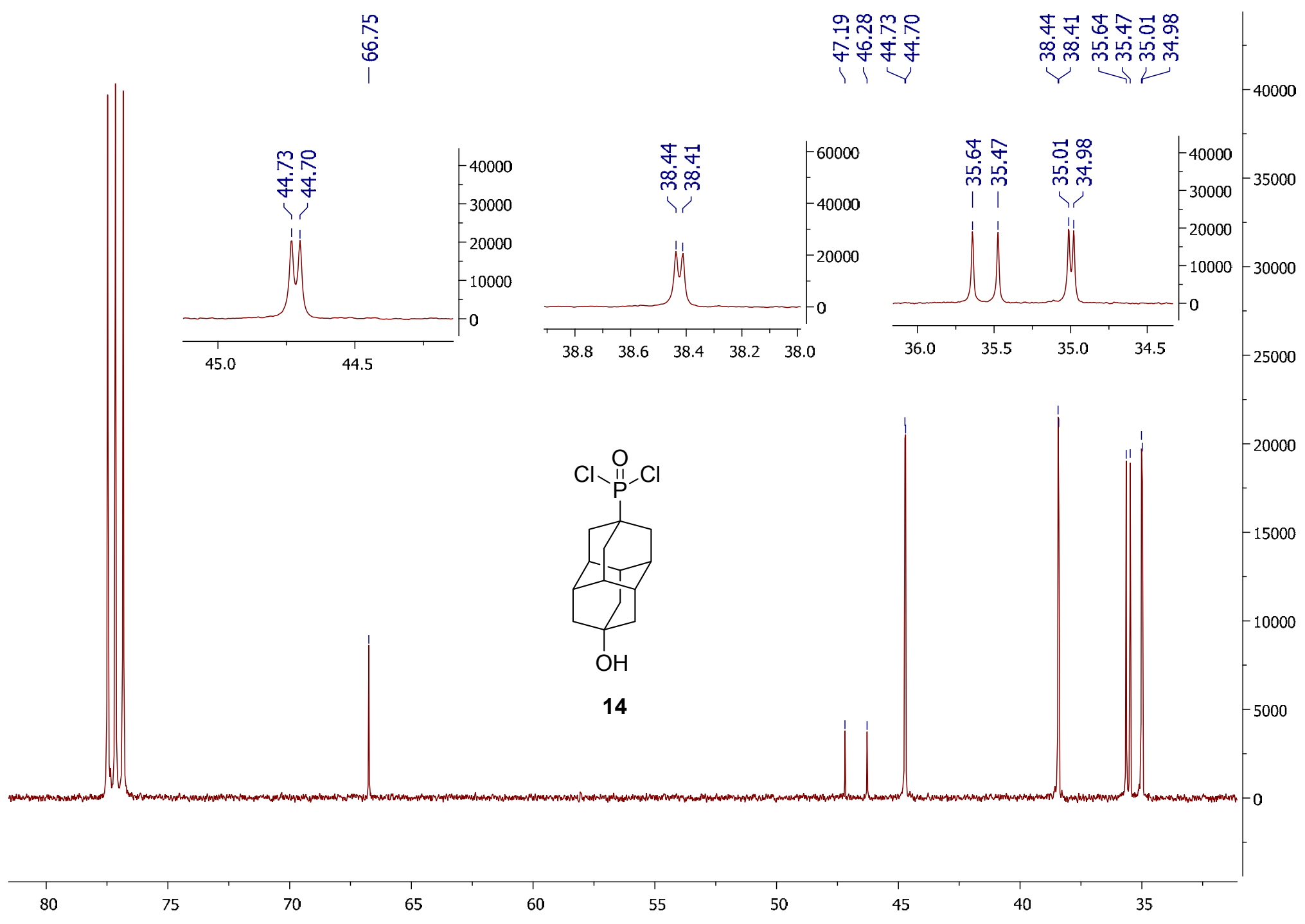


1.4.3. 9-OH-dia-4- $\mathrm{POCl}_{2}{ }^{31} \mathrm{P}$

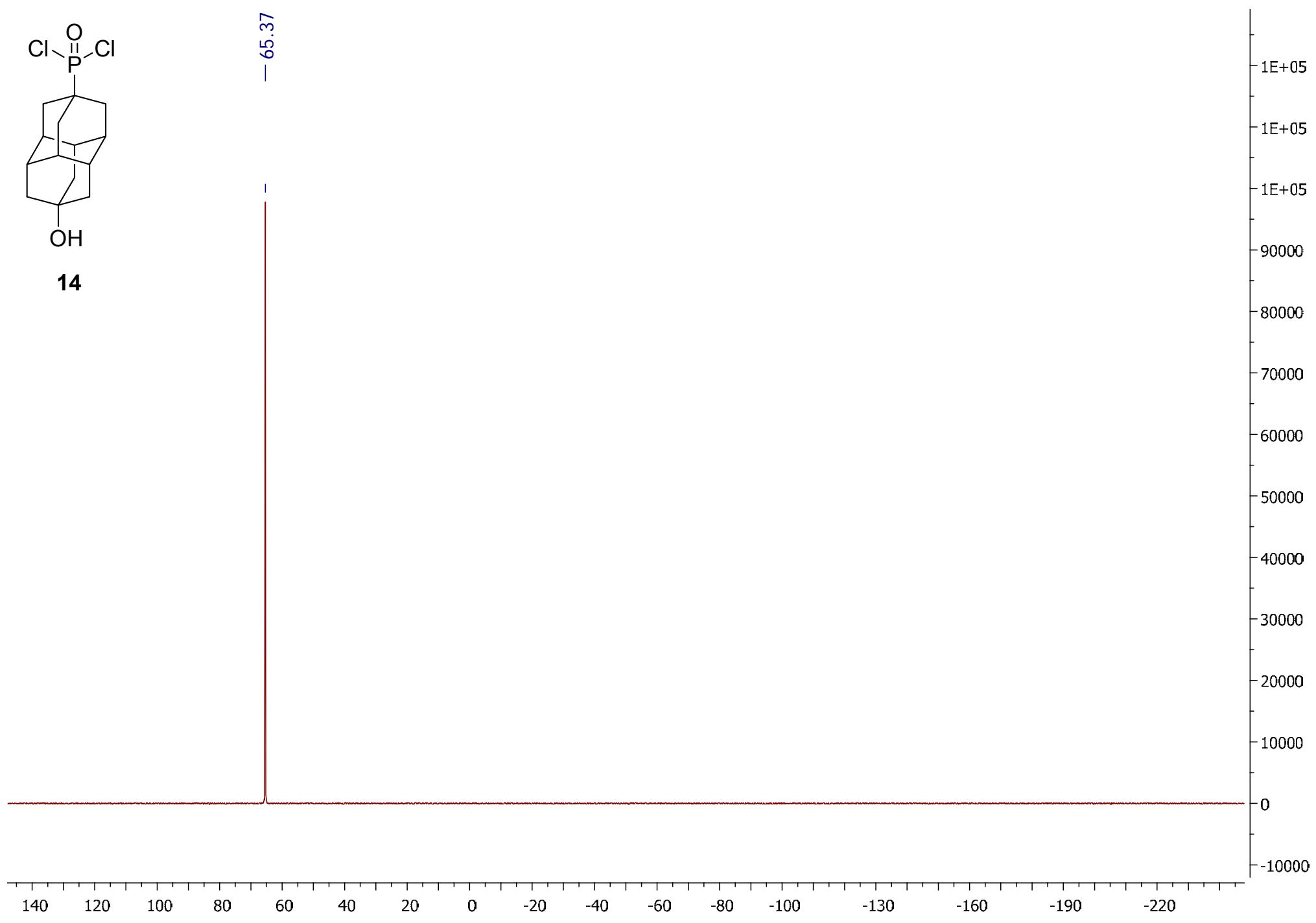


1.5. (3-Hydroxyadamant-1-yl)phosphonic dichloride (17)

1.5.1. $\quad 3-\mathrm{OH}$-ad-1-POCl ${ }_{2}{ }^{1} \mathrm{H}$

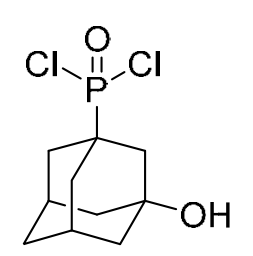

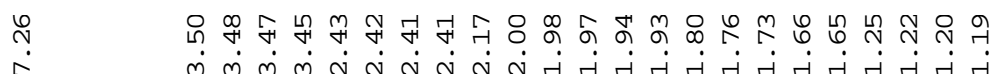

17
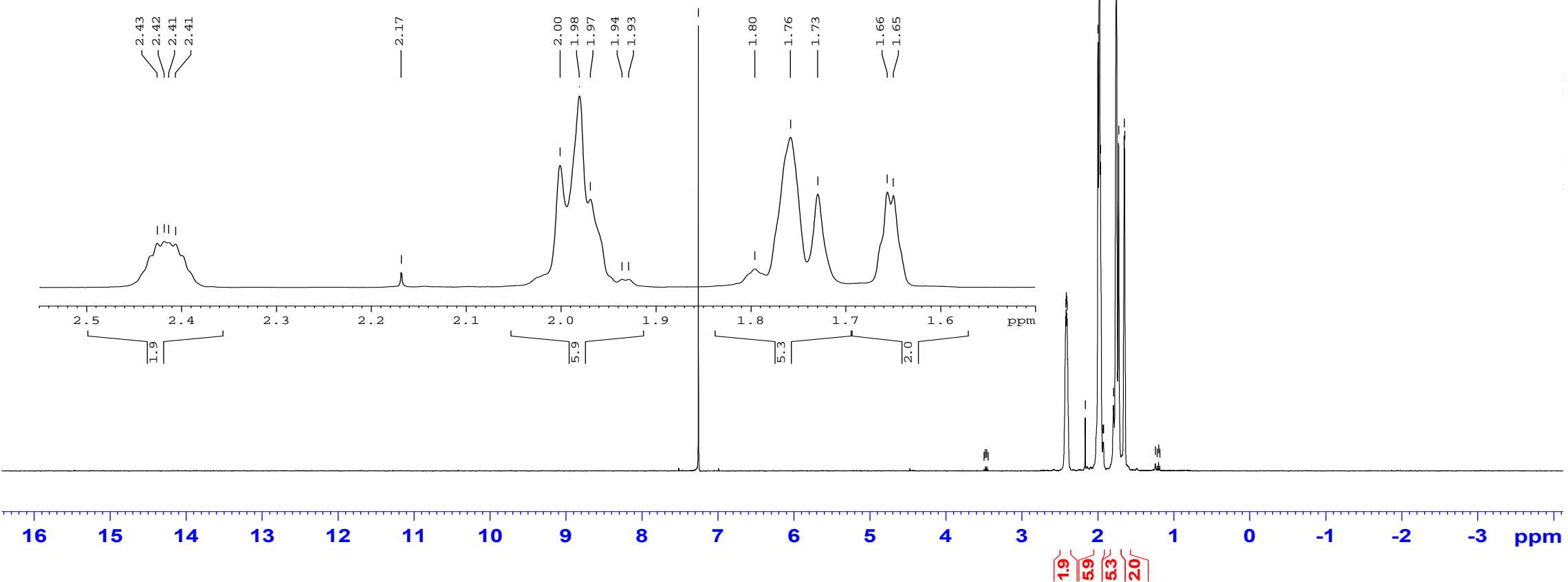
1.5.2. $3-\mathrm{OH}-\mathrm{ad}-1-\mathrm{POCl}_{2}{ }^{13} \mathrm{C}$

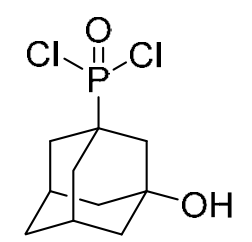

17
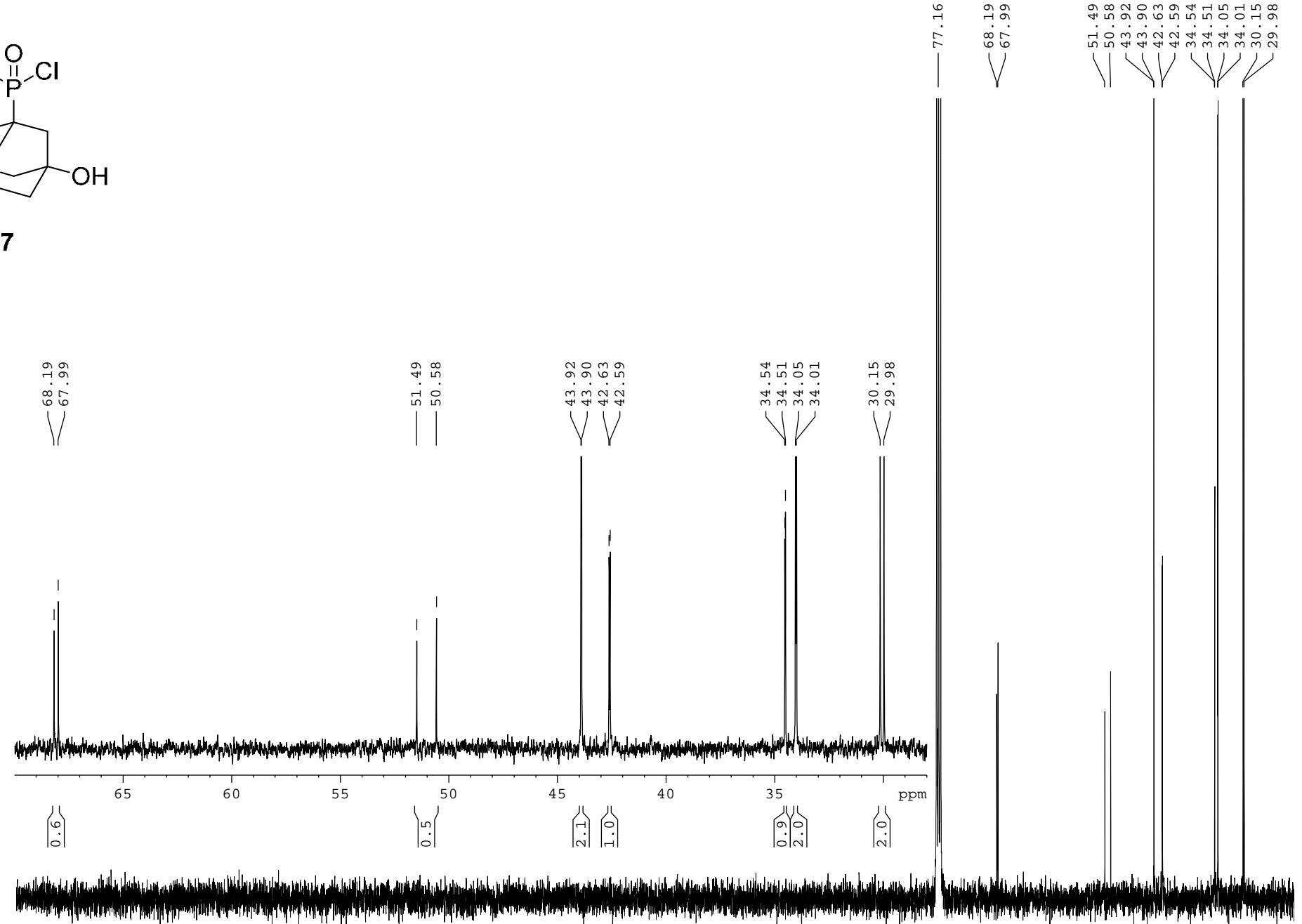

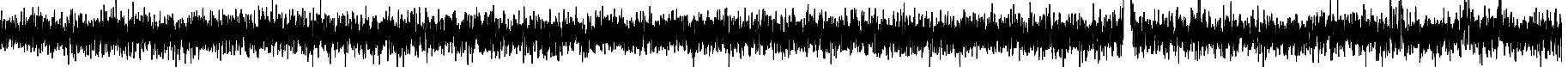

$\begin{array}{lllllllllllllllllll}210 & 200 & 190 & 180 & 170 & 160 & 150 & 140 & 130 & 120 & 110 & 100 & 90 & 80 & 70 & 60 & 50 & 40 & 30\end{array}$ 
1.5.3. $3-\mathrm{OH}-\mathrm{ad}-1-\mathrm{POCl}_{2}{ }^{31} \mathrm{P}$

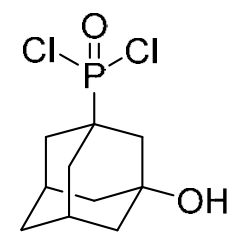

17

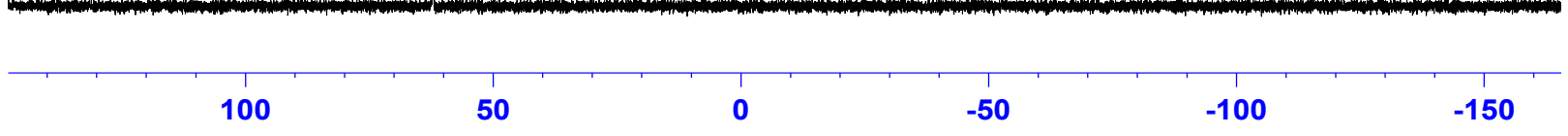


1.6. 4-Diamantylphosphonic acid dichloride (19)

1.6.1. 4-Dia- $\mathrm{POCl}_{2}{ }^{1} \mathrm{H}$

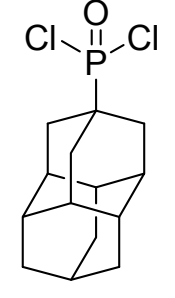

$\stackrel{\oplus}{m}$

เก

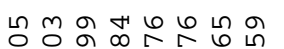

$\stackrel{\bullet}{\sim}$

$\hat{i}$

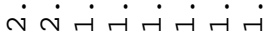

Sर

19
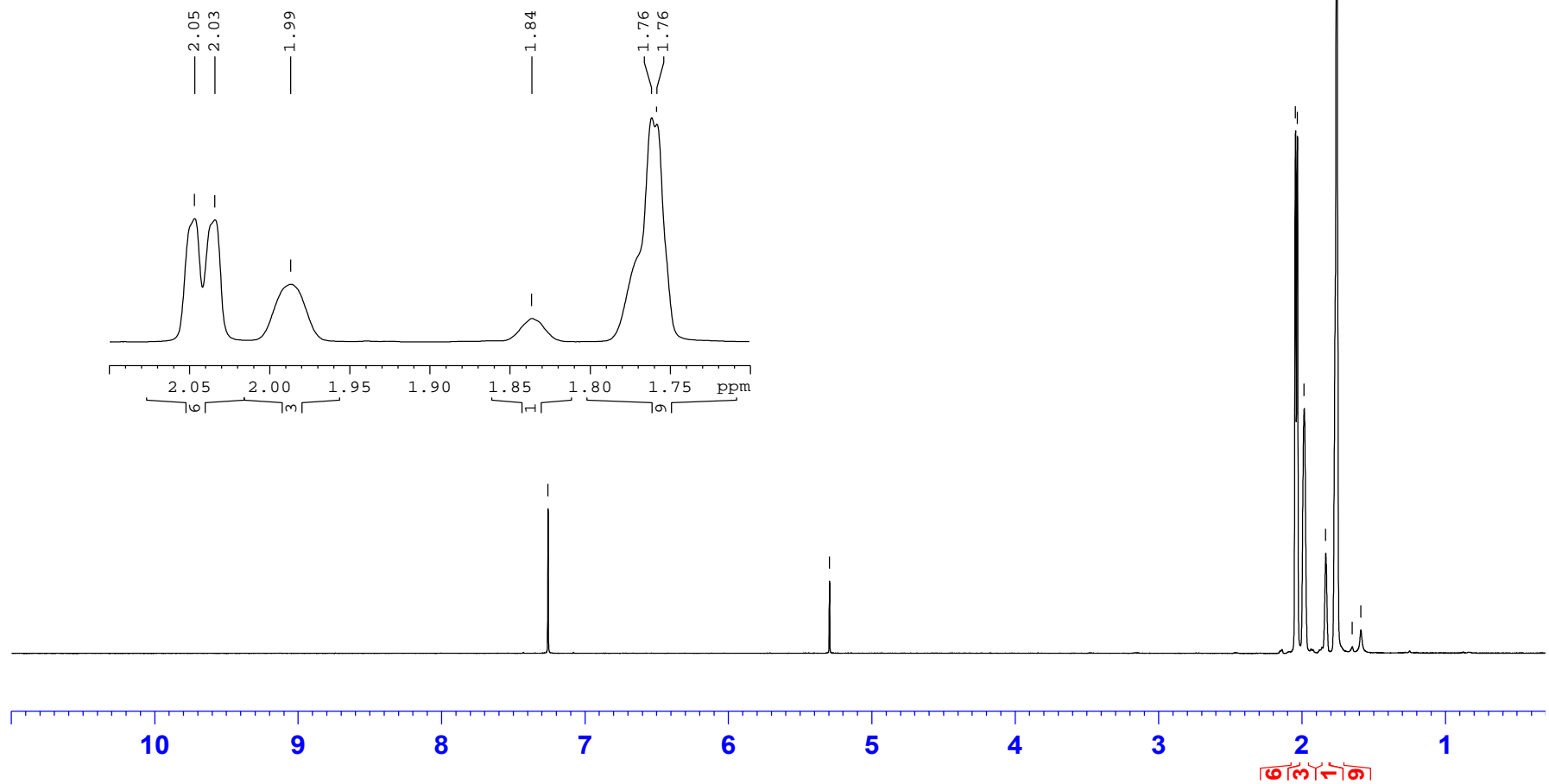
1.6.2. $\quad 4-\mathrm{Dia}-\mathrm{POCl}_{2}{ }^{13} \mathrm{C}$

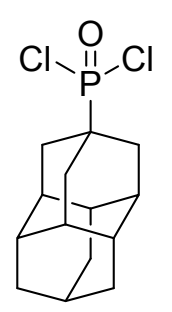

19
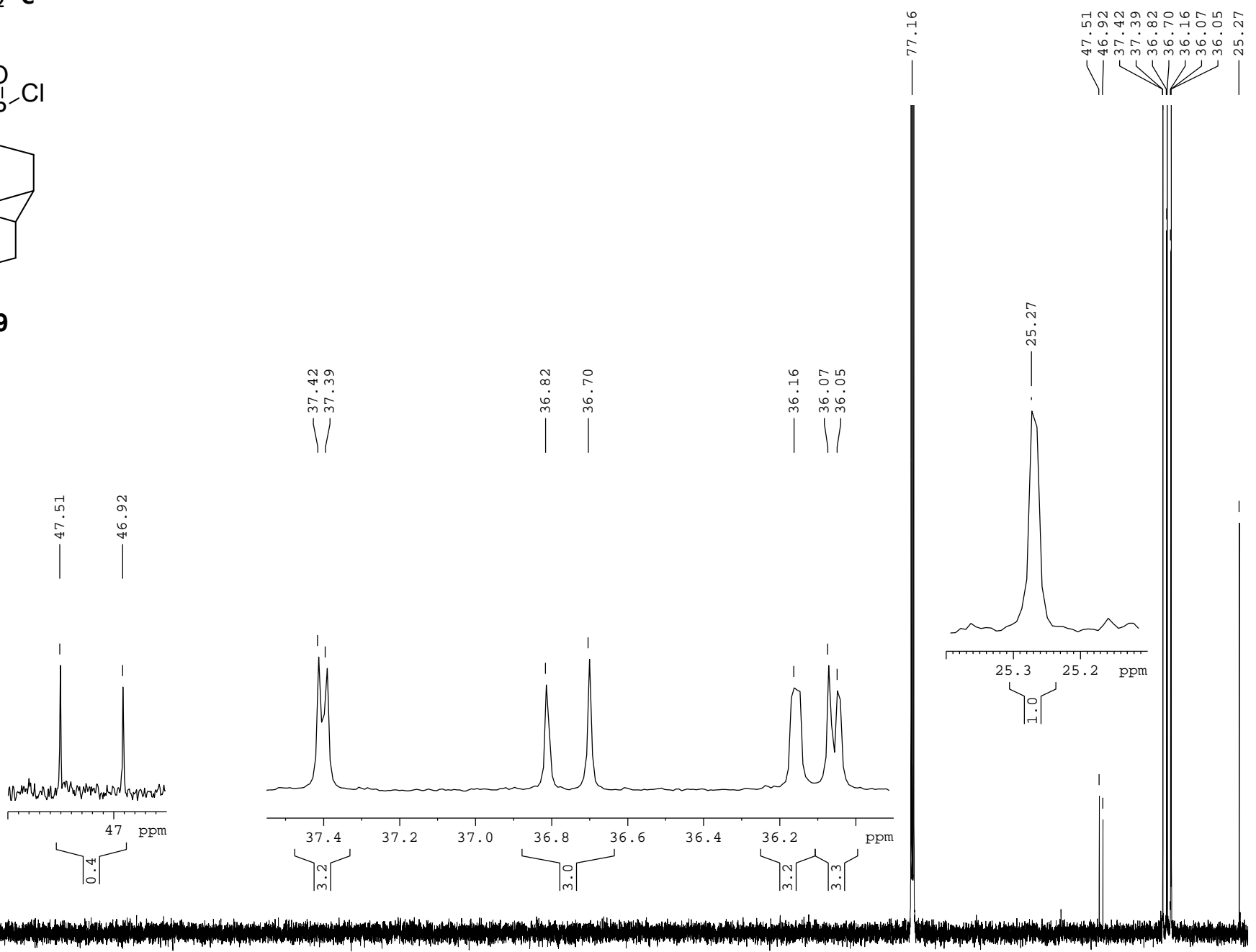

$\begin{array}{llllllllllllllllllll}220 & 210 & 200 & 190 & 180 & 170 & 160 & 150 & 140 & 130 & 120 & 110 & 100 & 90 & 80 & 70 & 60 & 50 & 40 & 30\end{array}$ 
1.6.3. 4-Dia- $\mathrm{POCl}_{2}{ }^{31} \mathrm{P}$

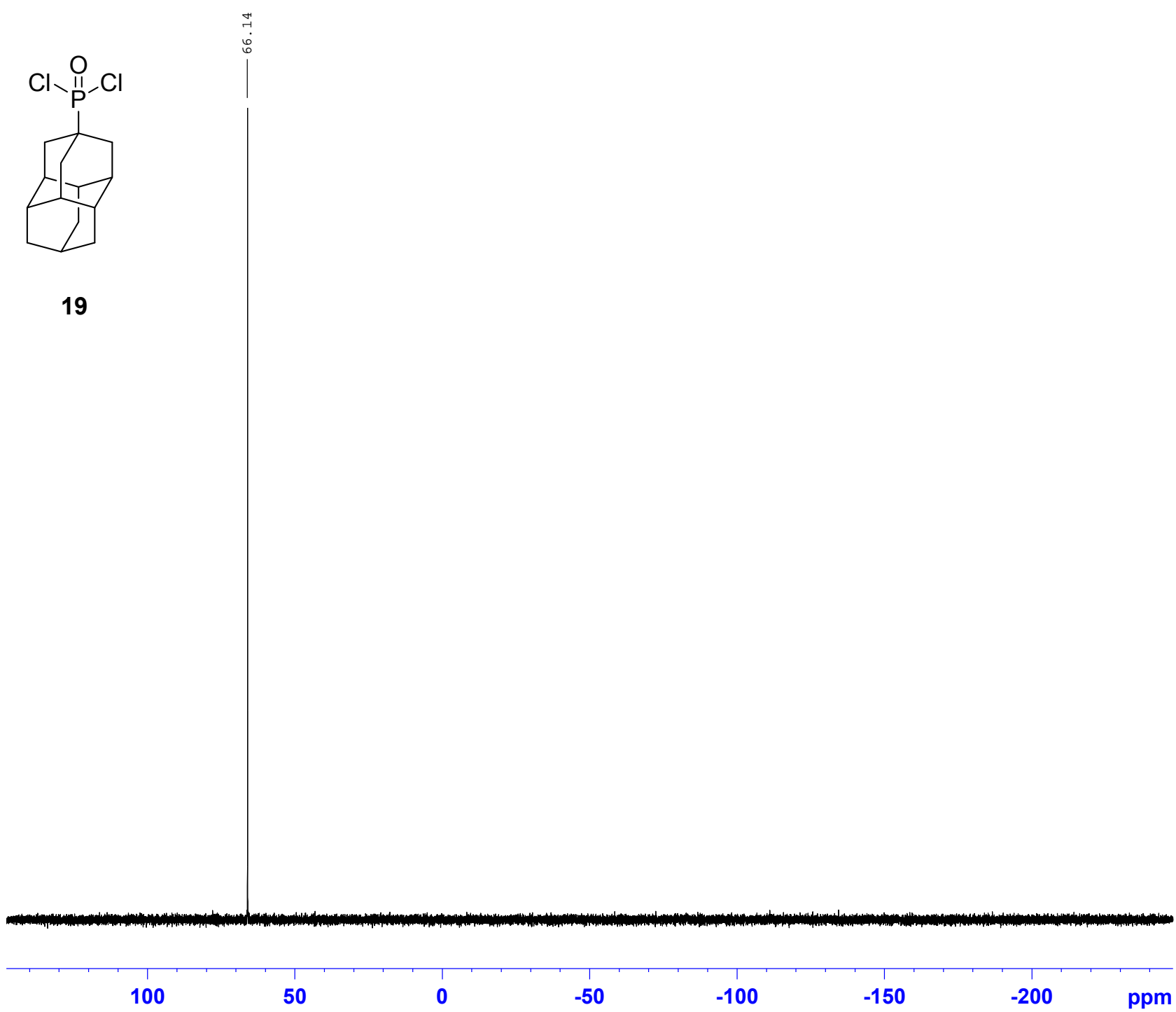


1.7. 1-Diamantylphosphonic acid dichloride (20)

1.7.1. 1-Dia-POCl${ }_{2}{ }^{1} \mathrm{H}$

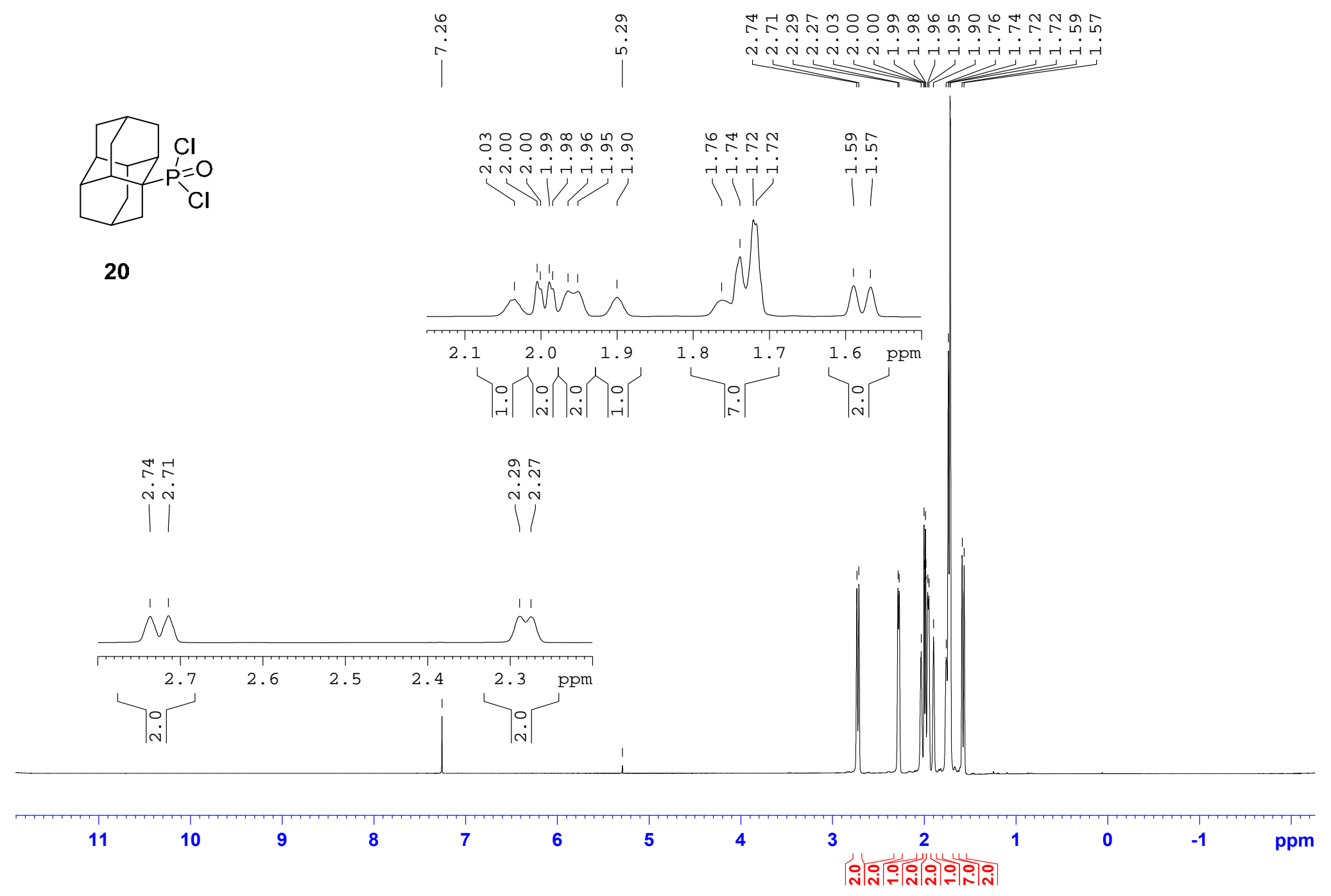


1.7.2. 1 -Dia- $\mathrm{POCl}_{2}{ }^{13} \mathrm{C}$
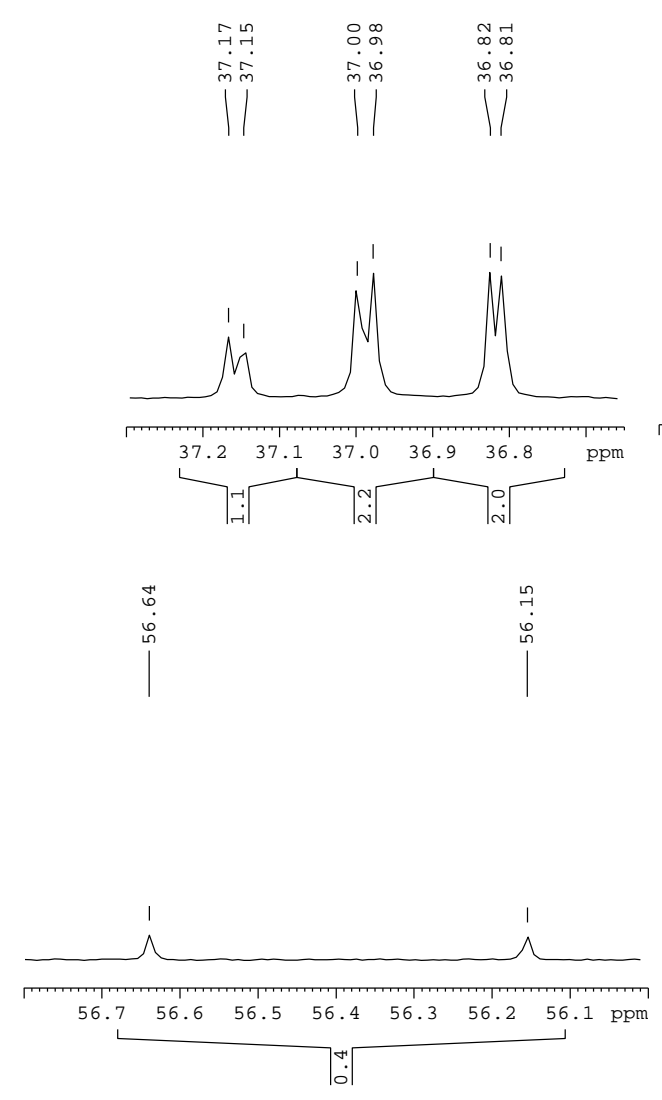
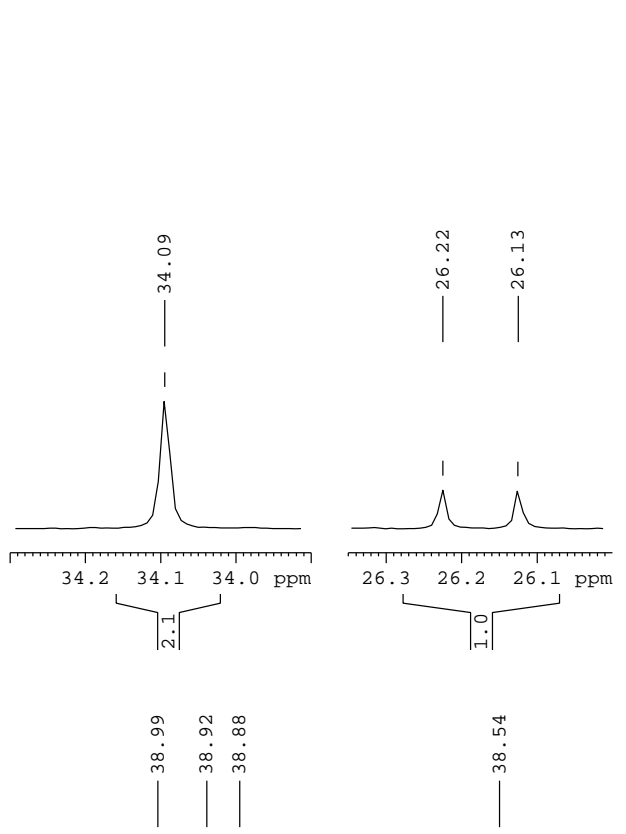

ร.

||$^{\infty} \mid$

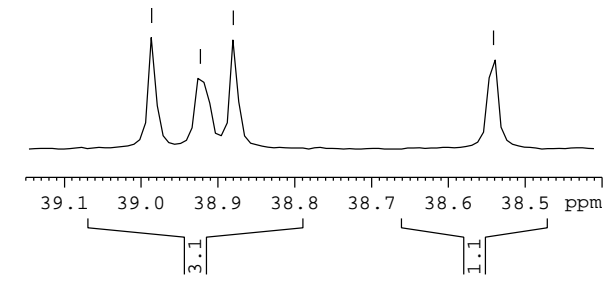

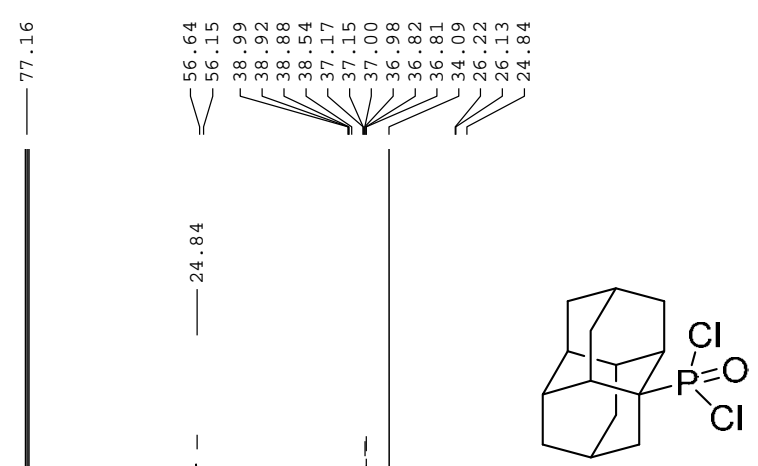

20

\footnotetext{
$\begin{array}{llllllllllllllllllllllllll}220 & 210 & 200 & 190 & 180 & 170 & 160 & 150 & 140 & 130 & 120 & 110 & 100 & 90 & 80 & 70 & 60 & 50 & 40 & 30 & 20 & 10 & 0 & \text { ppm }\end{array}$

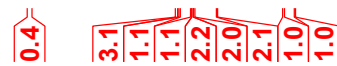


1.7.3. 1-Dia- $\mathrm{POCl}_{2}{ }^{31} \mathrm{P}$

1.7.4.

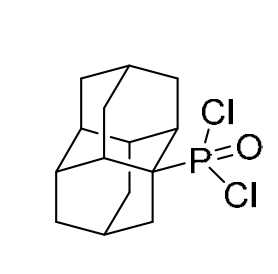

20

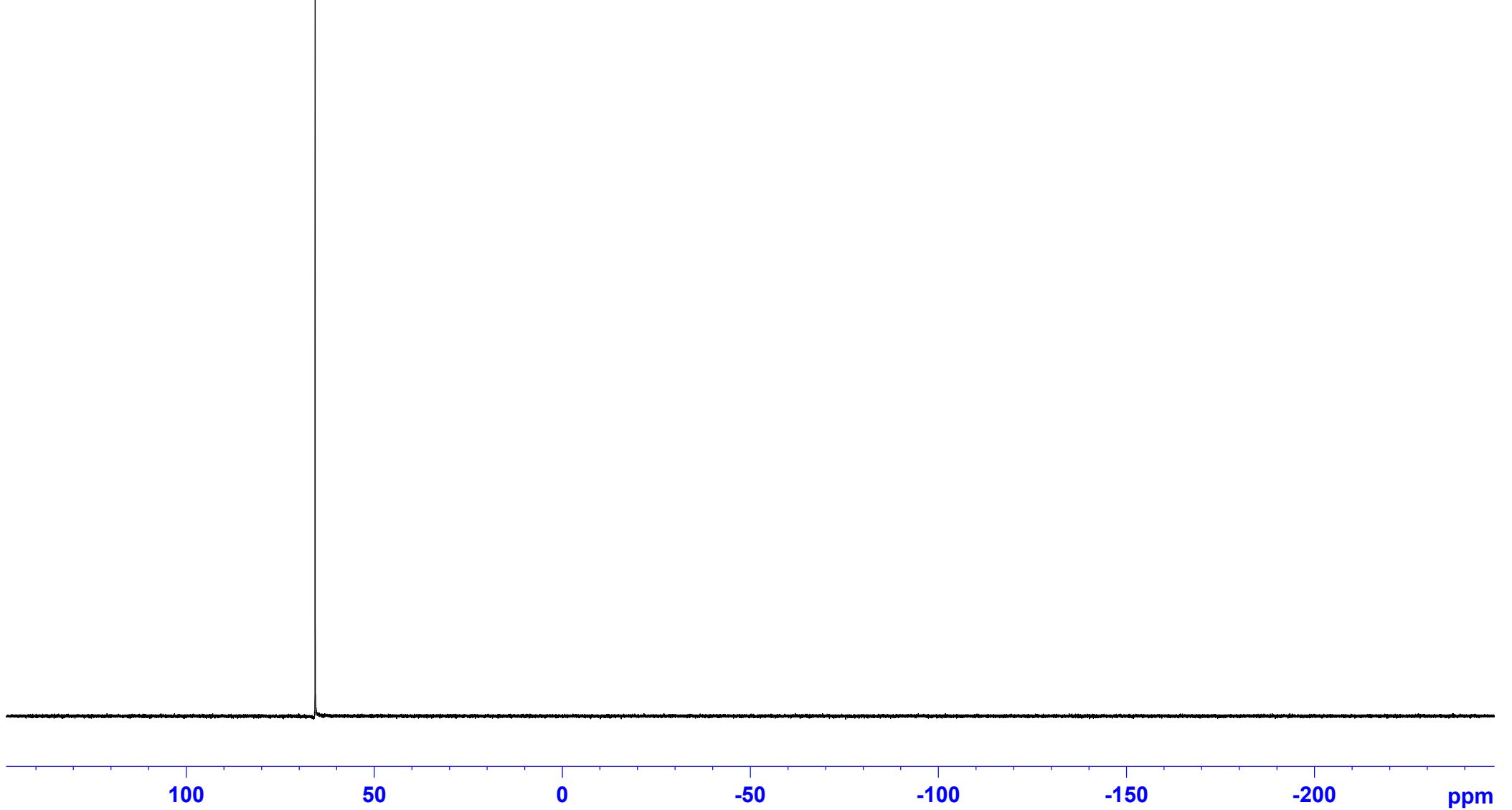


1.7.5. (4-Nitroxydiamant-1-yl)phosphonic dichloride (21)

1.7.6. 4- $\mathrm{ONO}_{2}$-dia-1- $\mathrm{POCl}_{2}{ }^{1} \mathrm{H}$

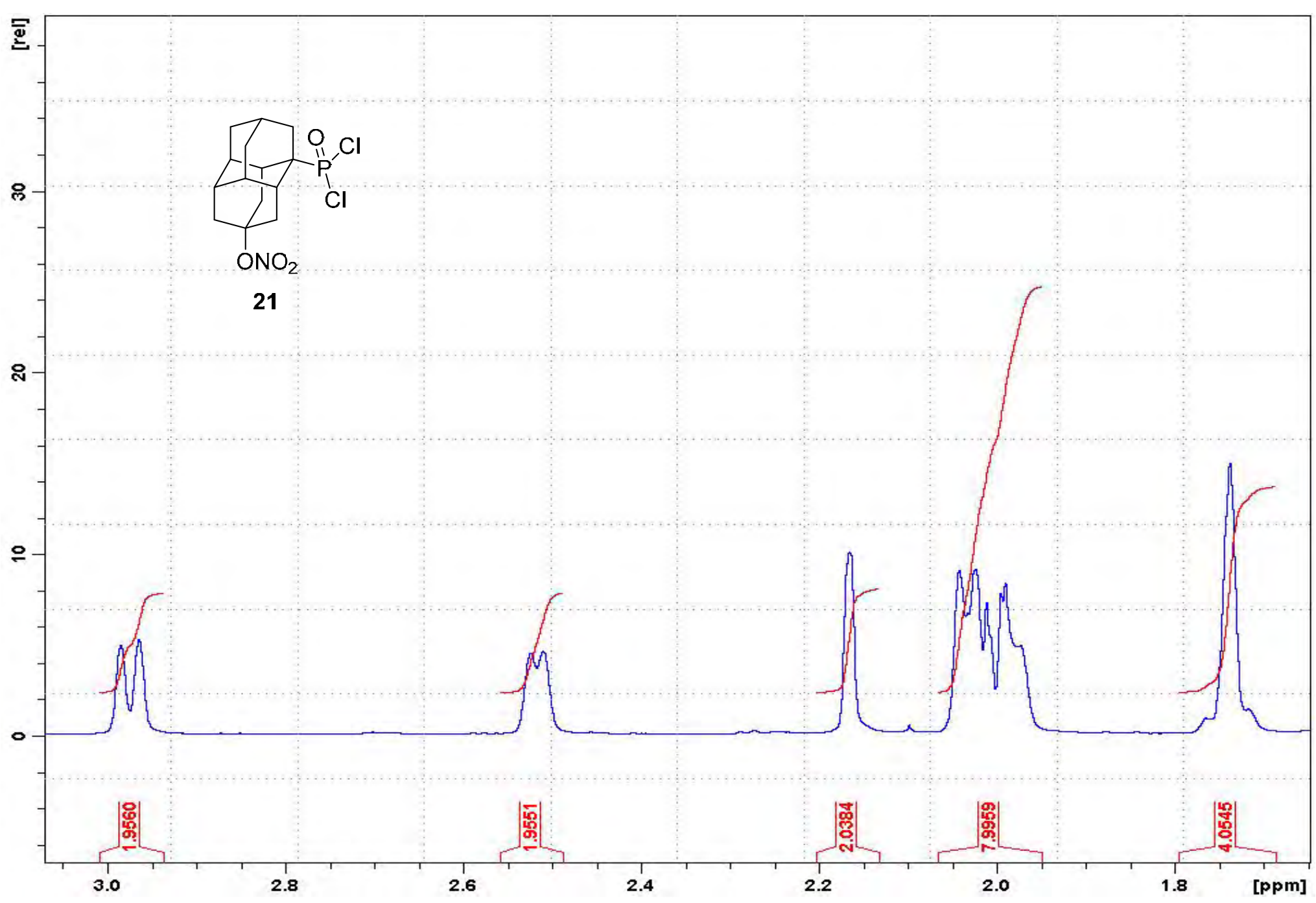


1.7.7. $4-\mathrm{ONO}_{2}$-dia-1- $\mathrm{POCl}_{2}{ }^{13} \mathrm{C}$

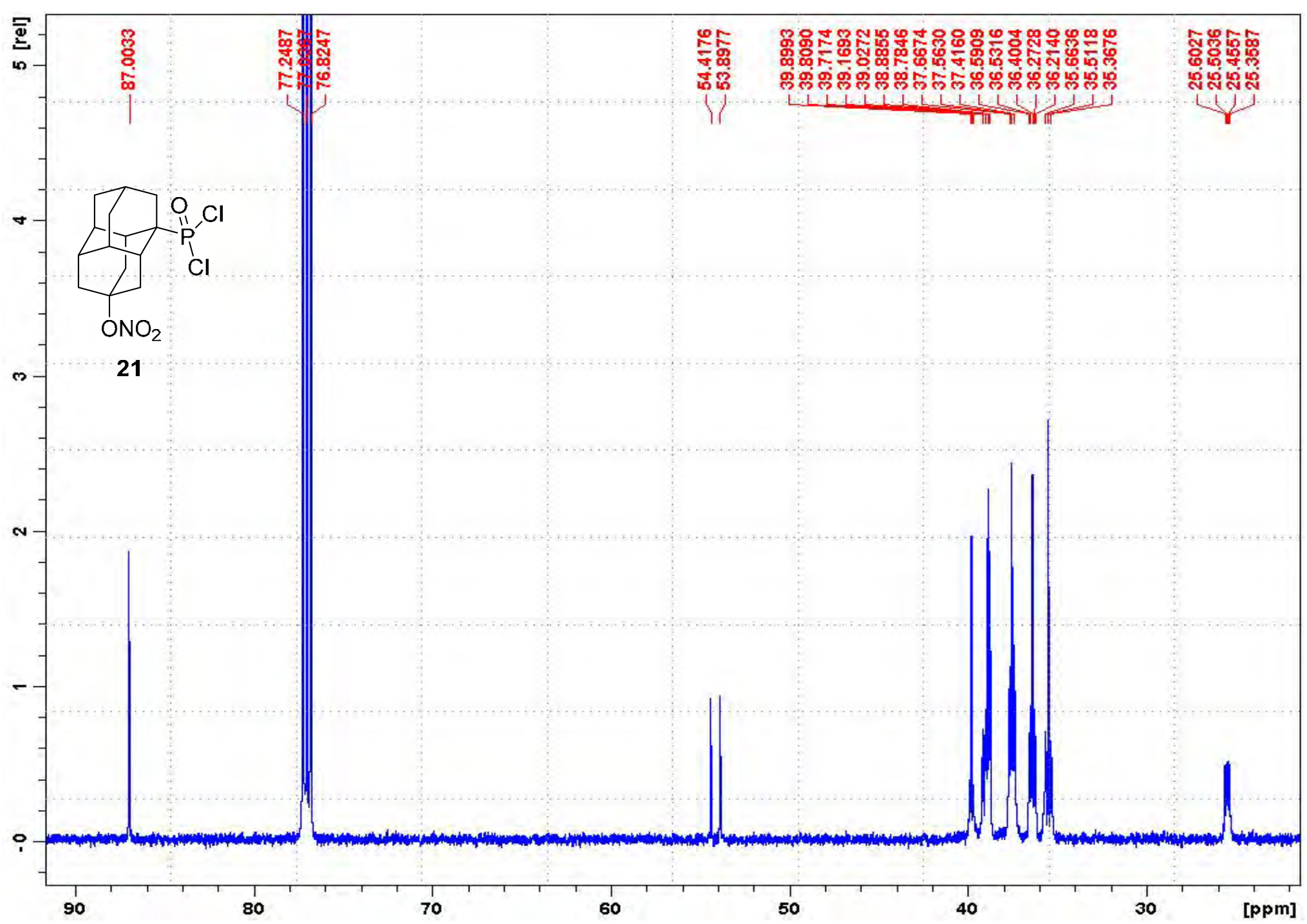


1.7.8. 4- $\mathrm{ONO}_{2}$-dia-1- $\mathrm{POCl}_{2}{ }^{31} \mathrm{P}$

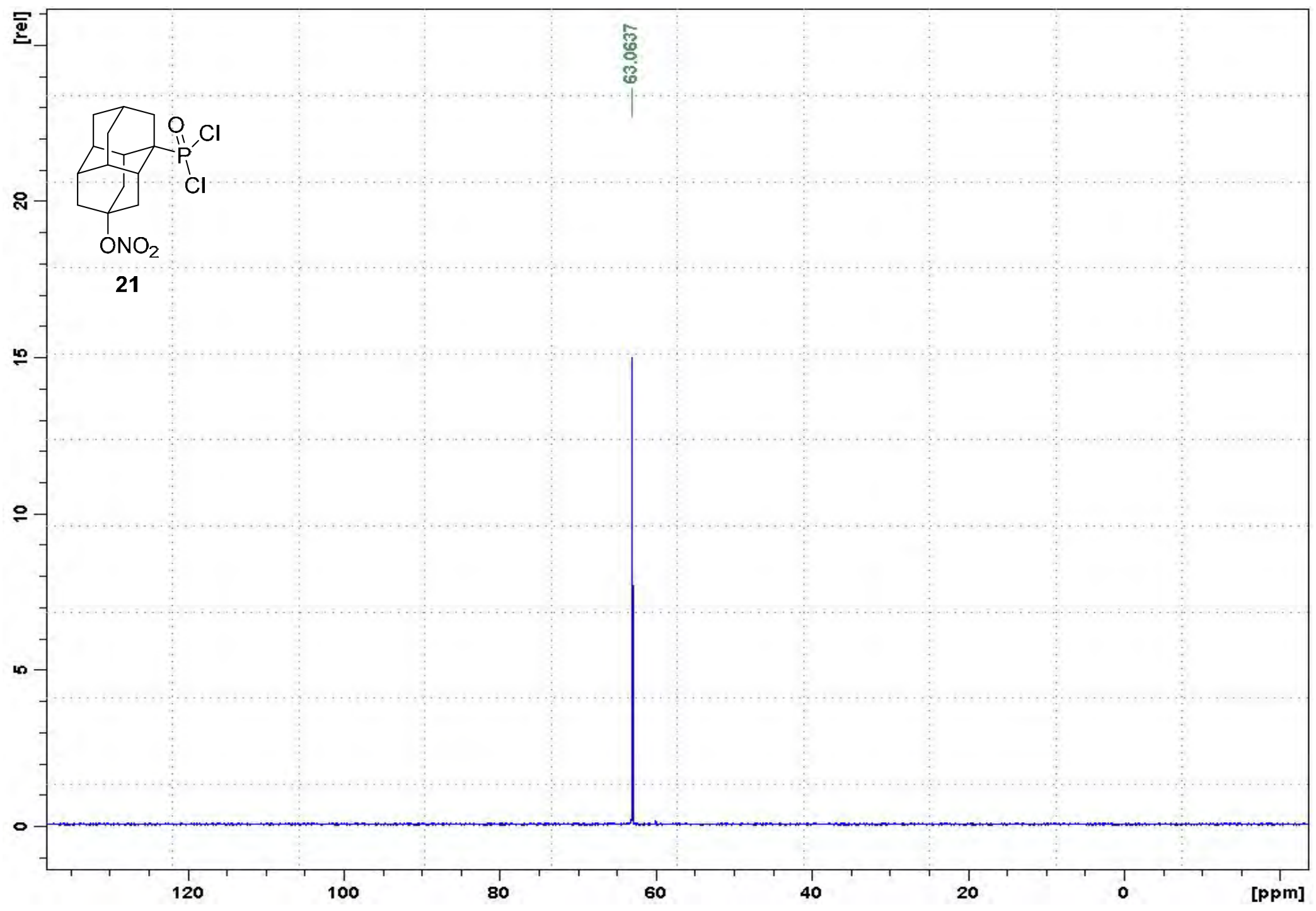


1.7.9. (6-Hydroxydiamant-1yl)phosphonic dichloride (24)

1.7.10. 6-OH-dia-1- $\mathrm{POCl}_{2}{ }^{1} \mathrm{H}$

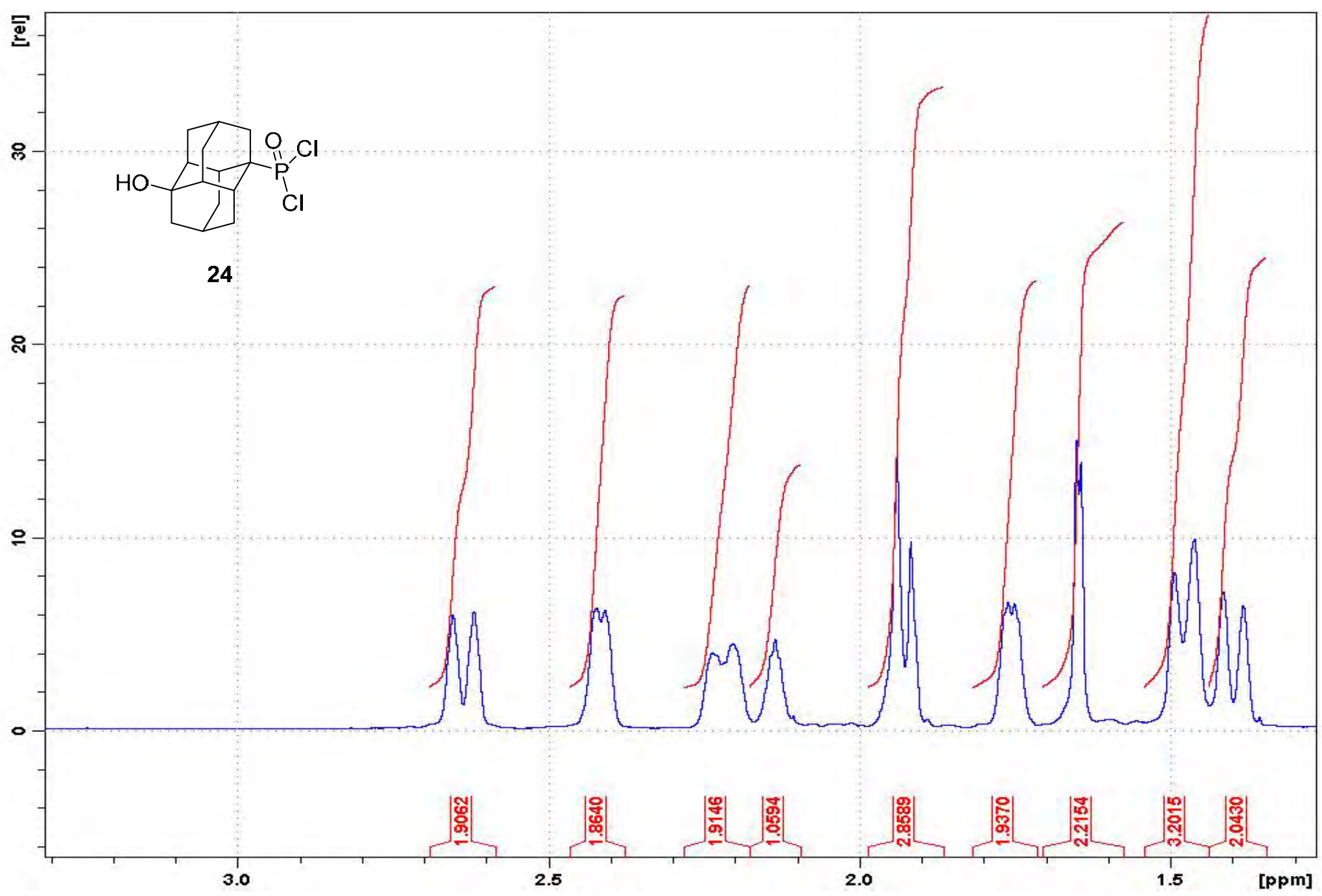


1.7.11. 6-OH-dia-1-POCl${ }_{2}^{13} \mathrm{C}$

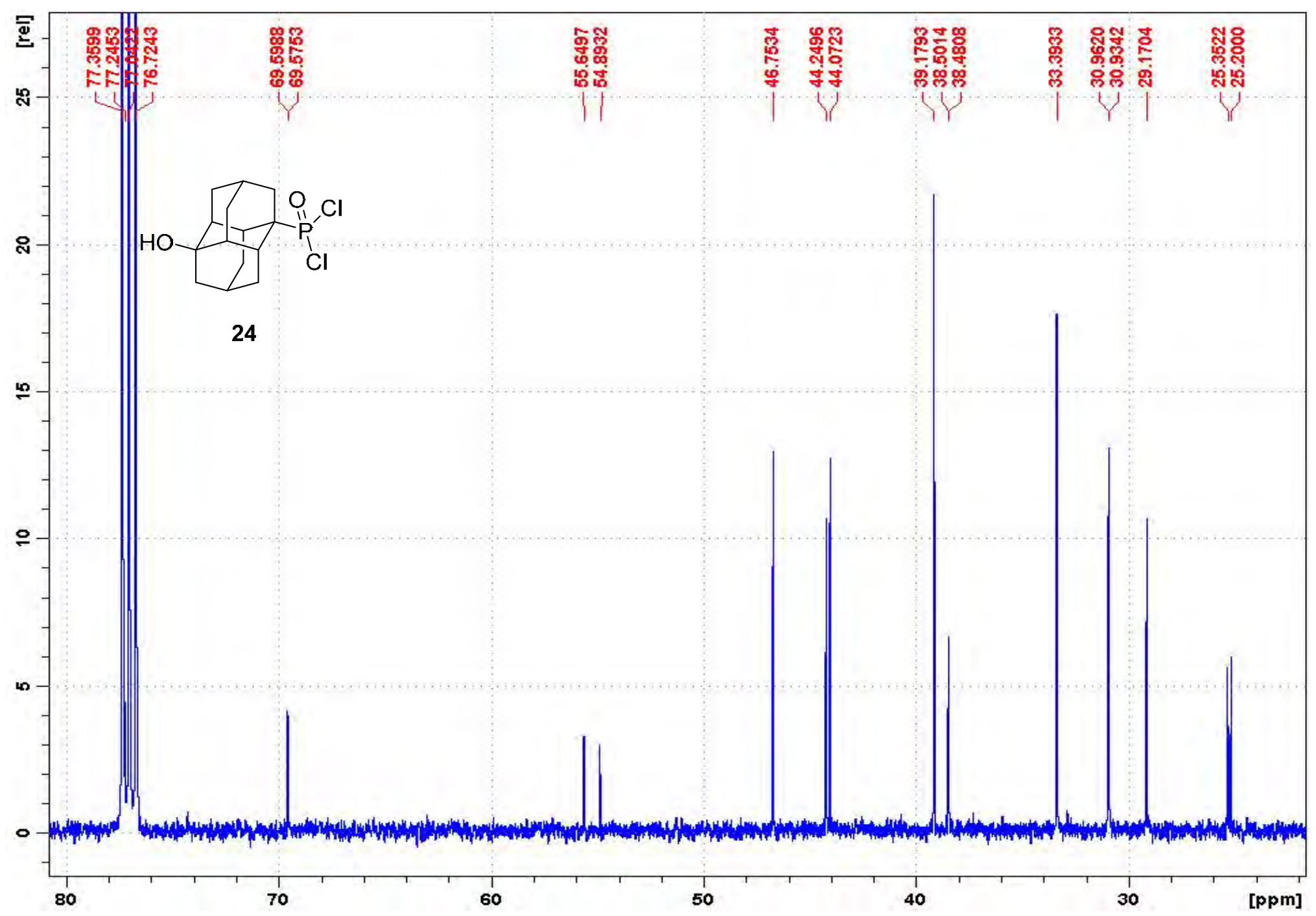


1.7.12. 6-OH-dia-1- $\mathrm{POCl}_{2}{ }^{31} \mathrm{P}$

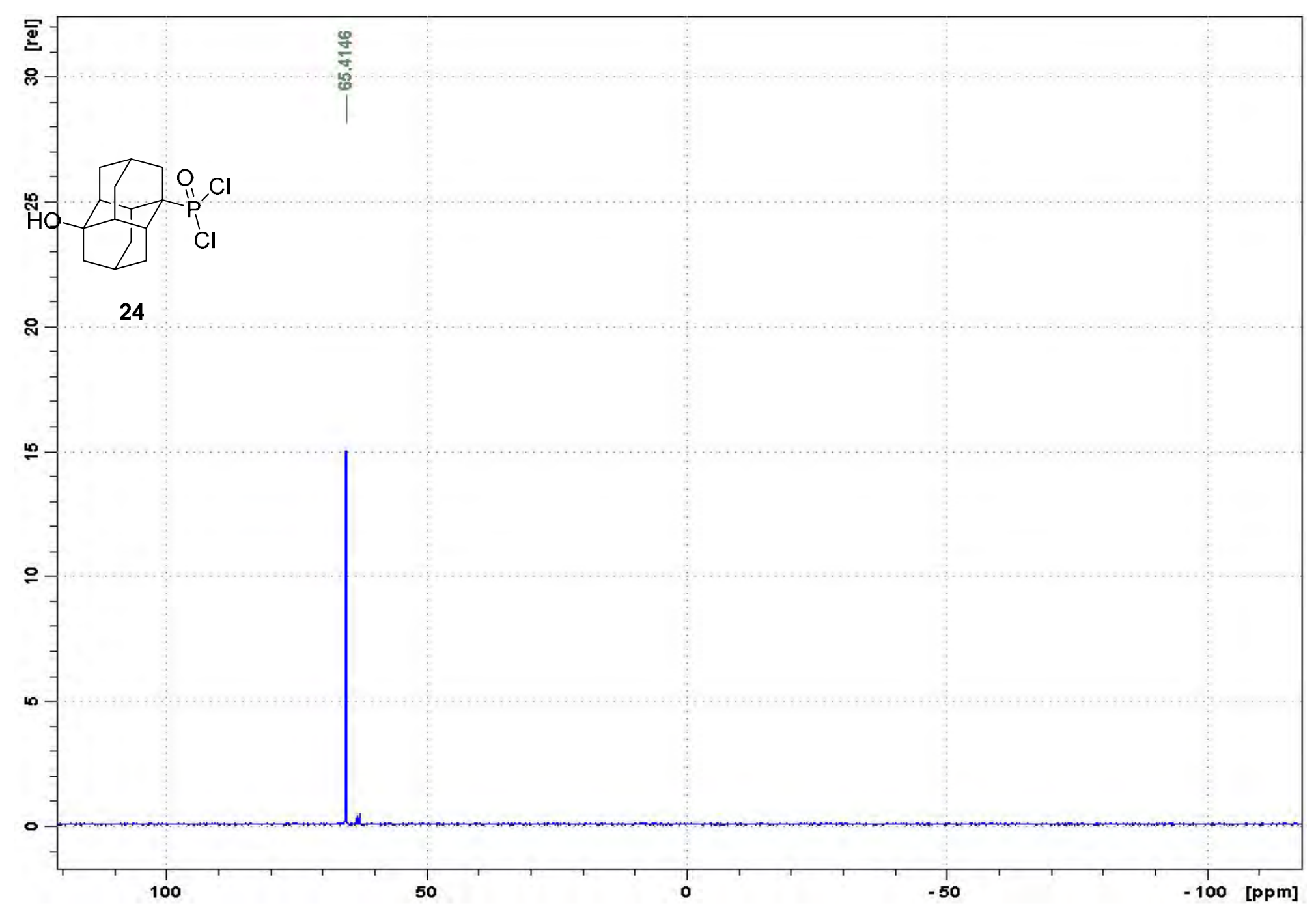


1.8. 4-Diamantylphenylchlorophosphinate (25)

1.8.1. 4-Dia-P(O)CIPh ${ }^{1} \mathrm{H}$

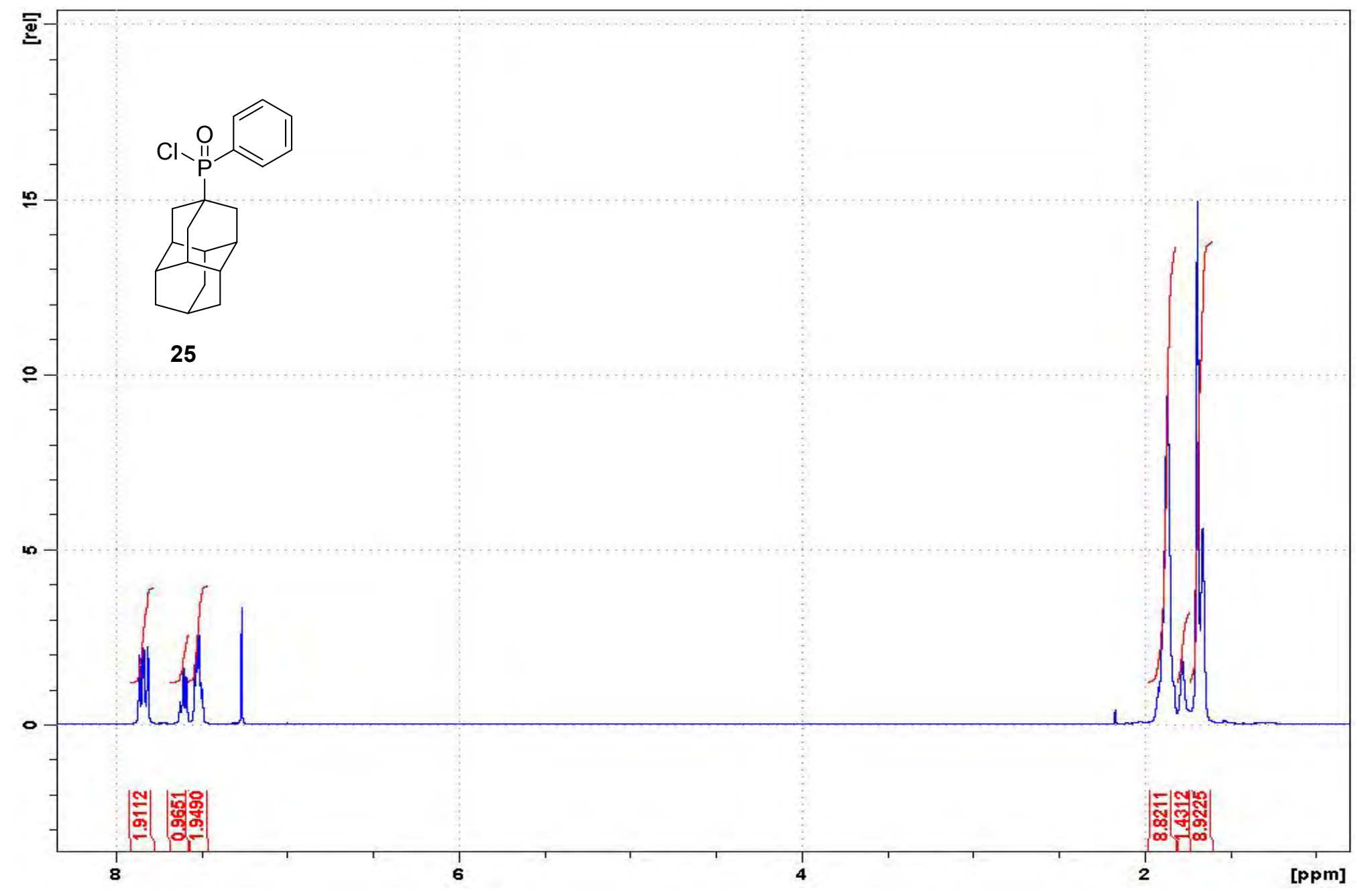


1.8.2. 4-Dia-P(O)CIPh ${ }^{13} \mathrm{C}$

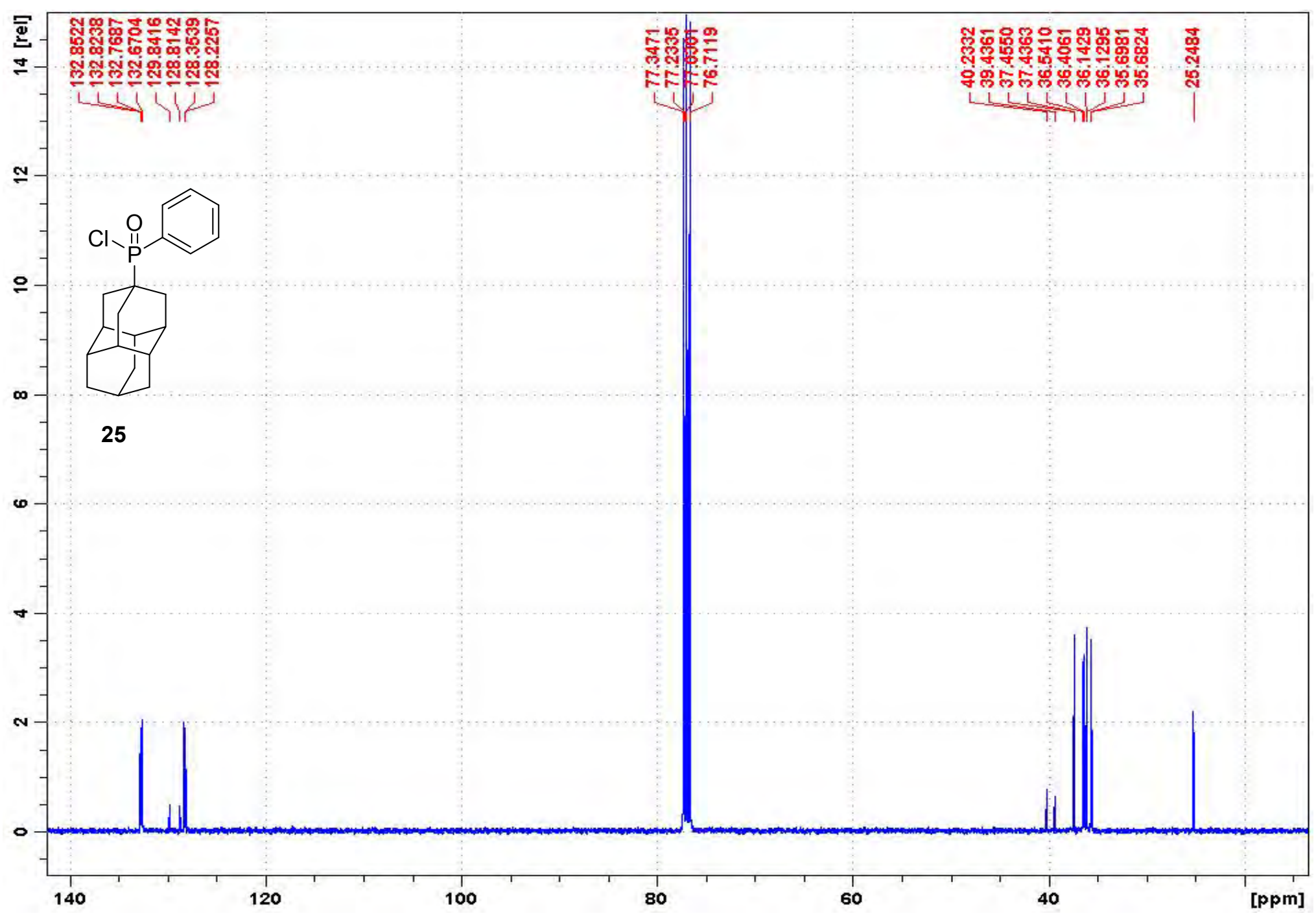


1.8.3. 4-Dia-P(O) $\mathrm{ClPh}^{31} \mathrm{P}$

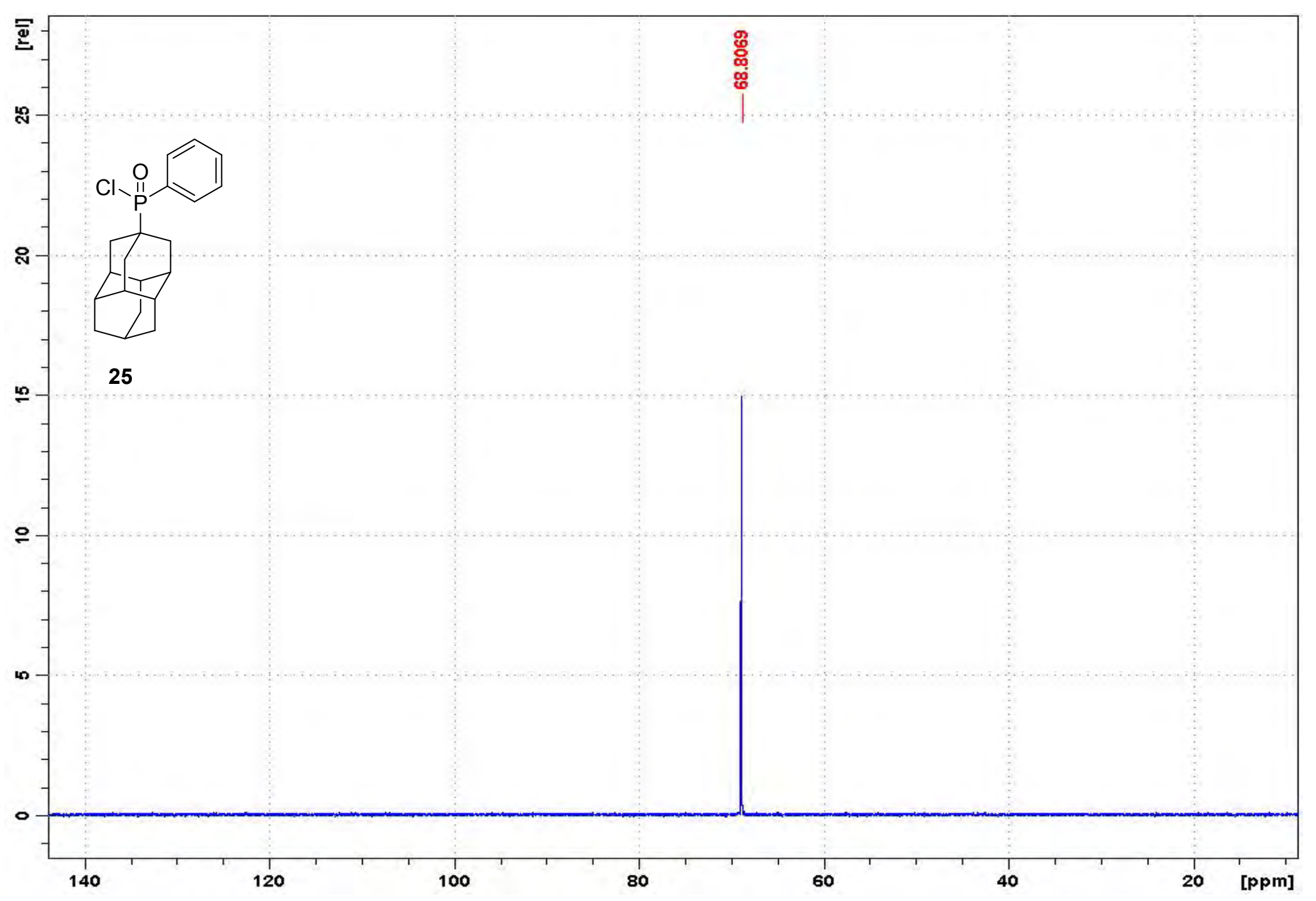


1.9. 1-Diamantylphenylchlorophosphonate (27)

1.9.1. 1-Dia-P(O)CIPh ${ }^{1} \mathrm{H}$

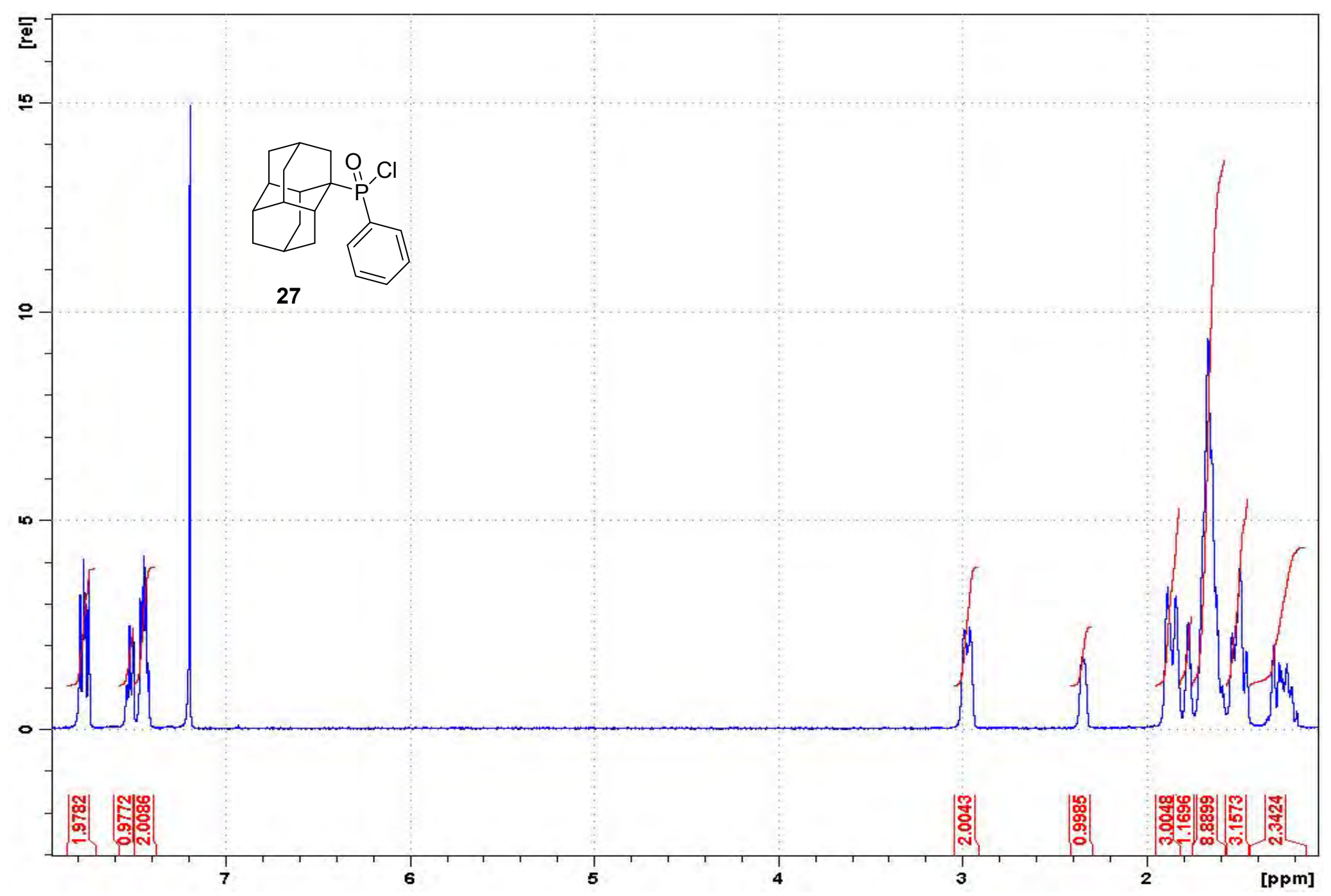


1.9.2. 1-Dia-P(O)CIPh ${ }^{13} \mathrm{C}$

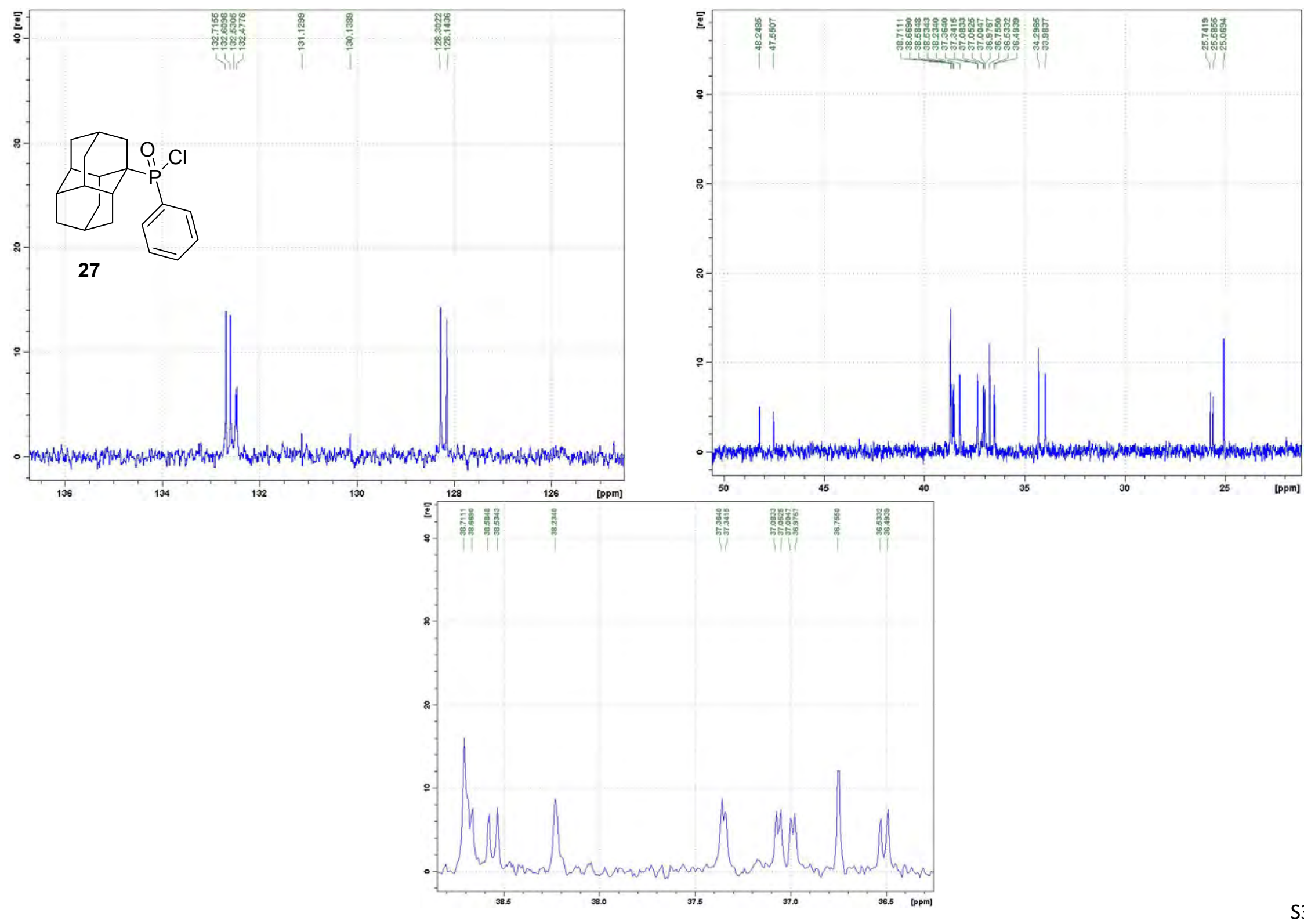


1.9.3. 1-Dia-P(O) $\mathrm{CIPh}^{31} \mathrm{P}$

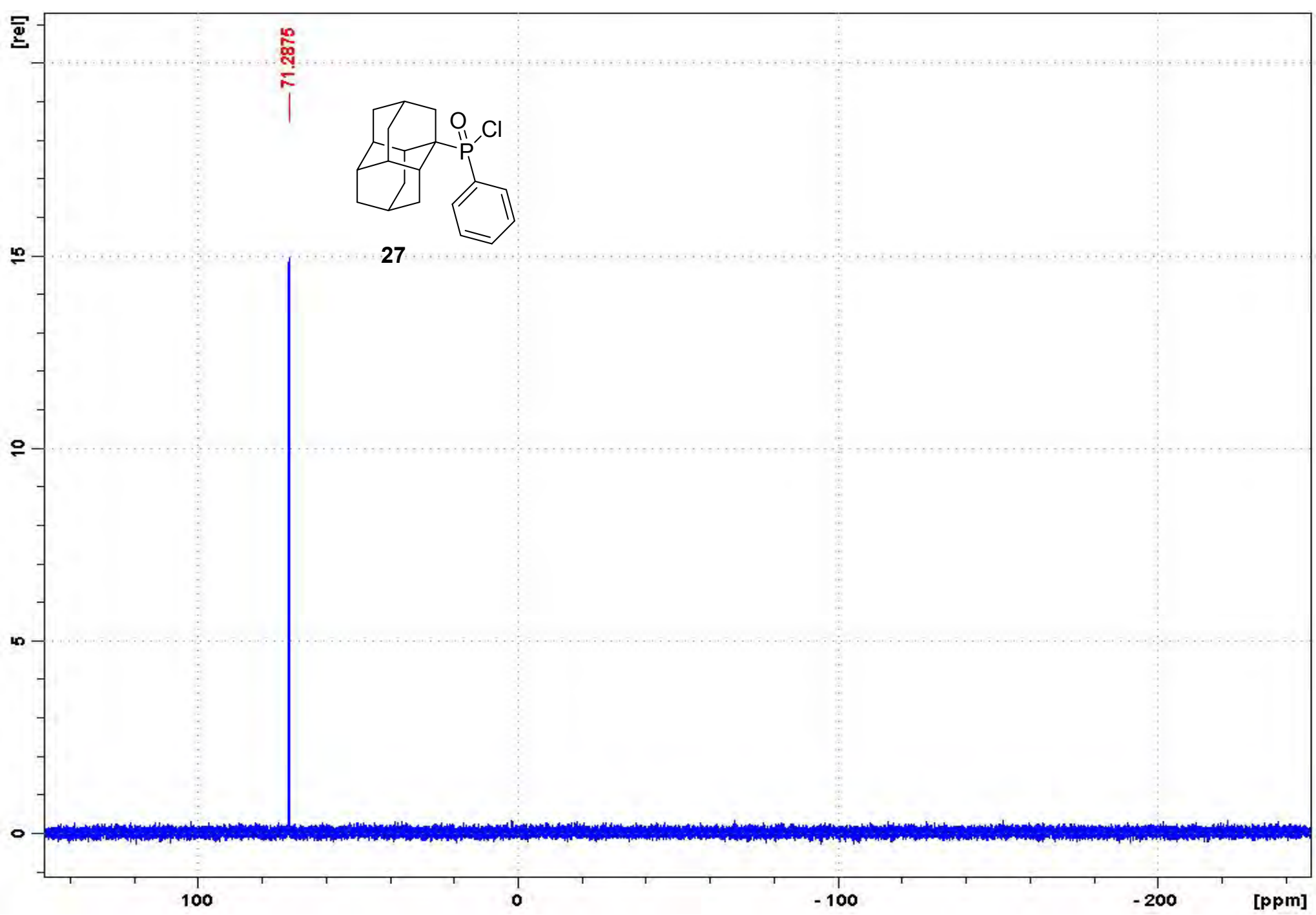


1.10. 4-Diamantylphosphine (29)

1.10.1. 4-Dia- $\mathrm{PH}_{2}{ }^{1} \mathrm{H}$

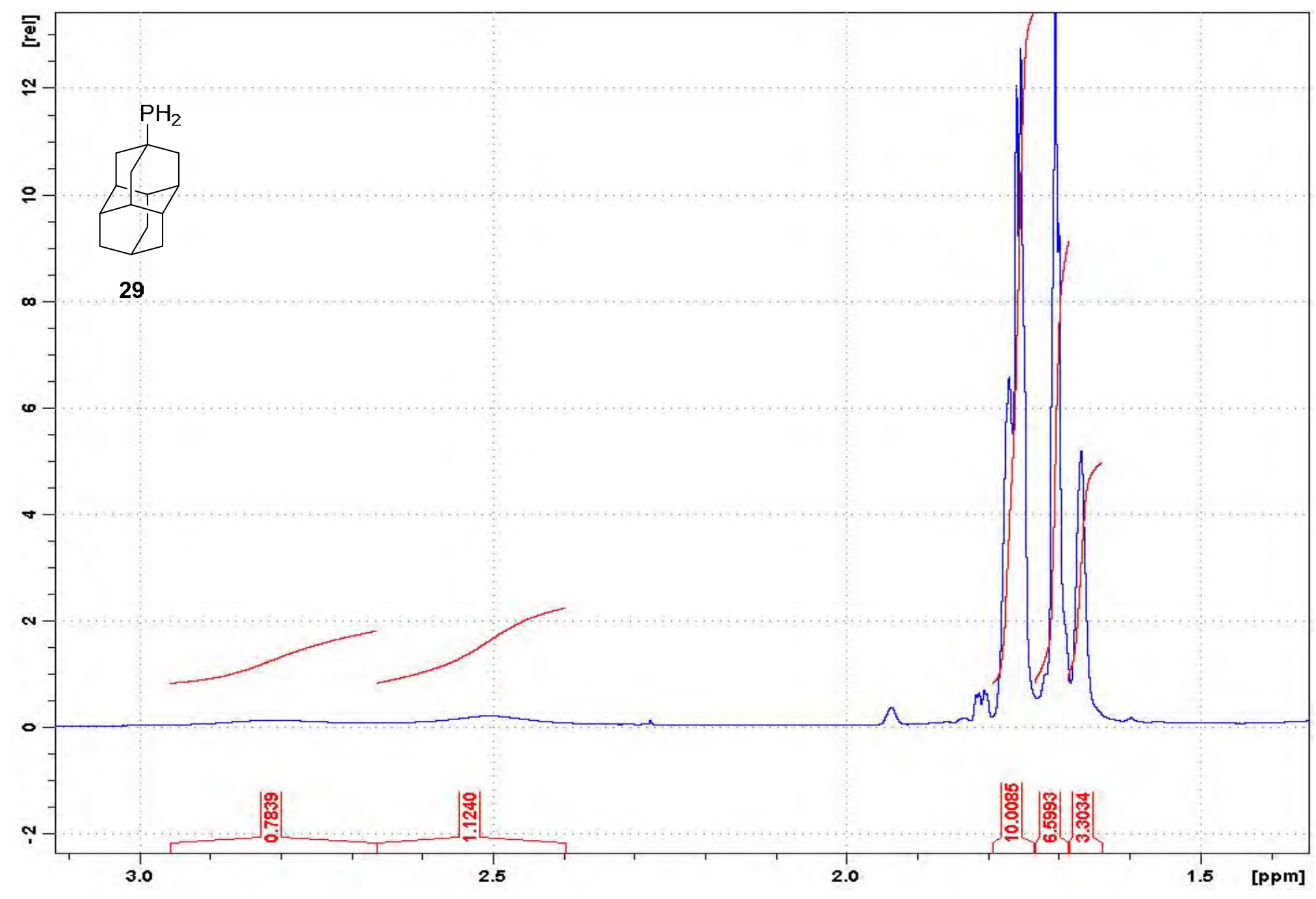


1.10.2. 4-Dia- $\mathrm{PH}_{2}{ }^{13} \mathrm{C}$

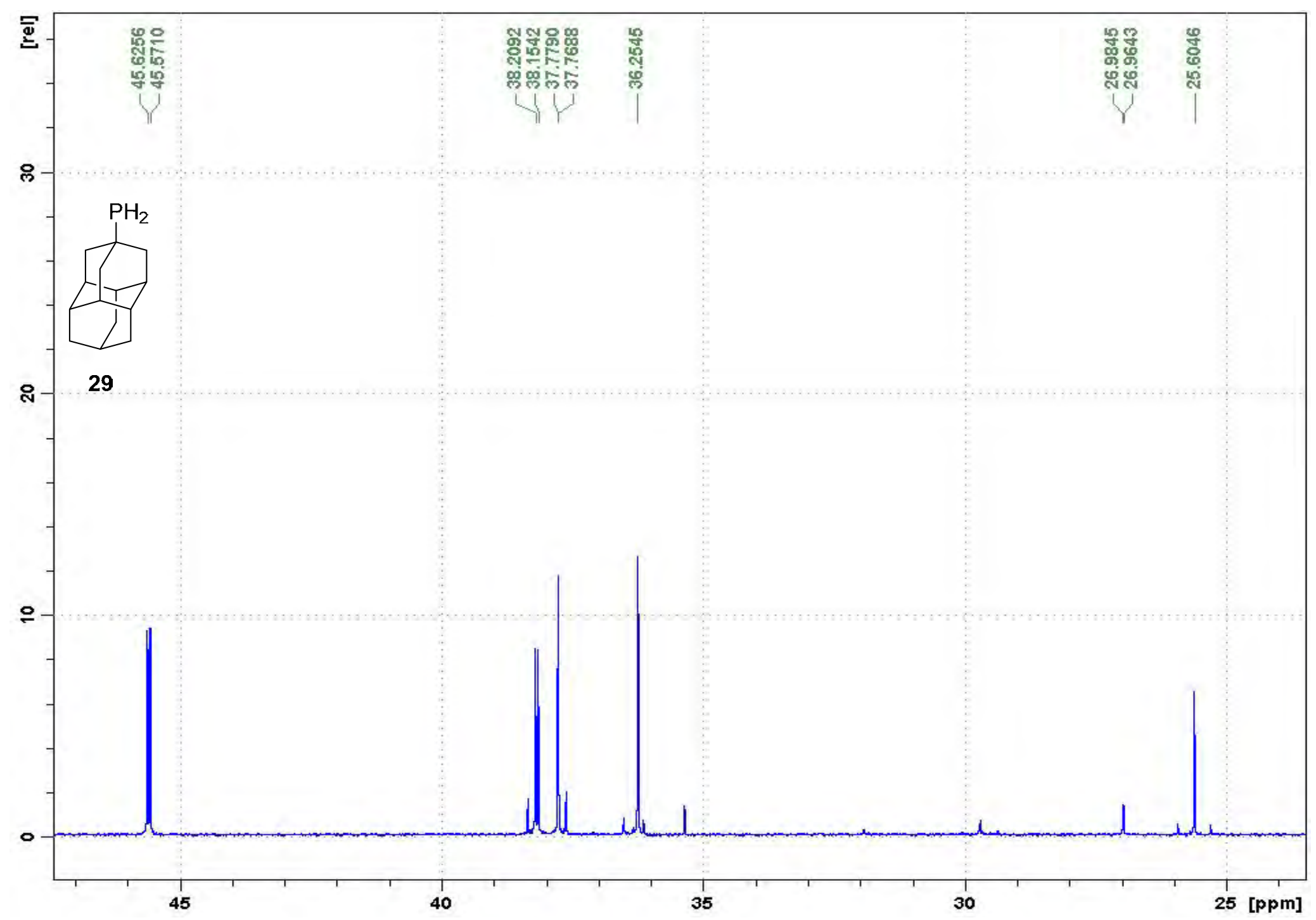


1.10.3. 4-Dia- $\mathrm{PH}_{2}{ }^{31} \mathrm{P}$

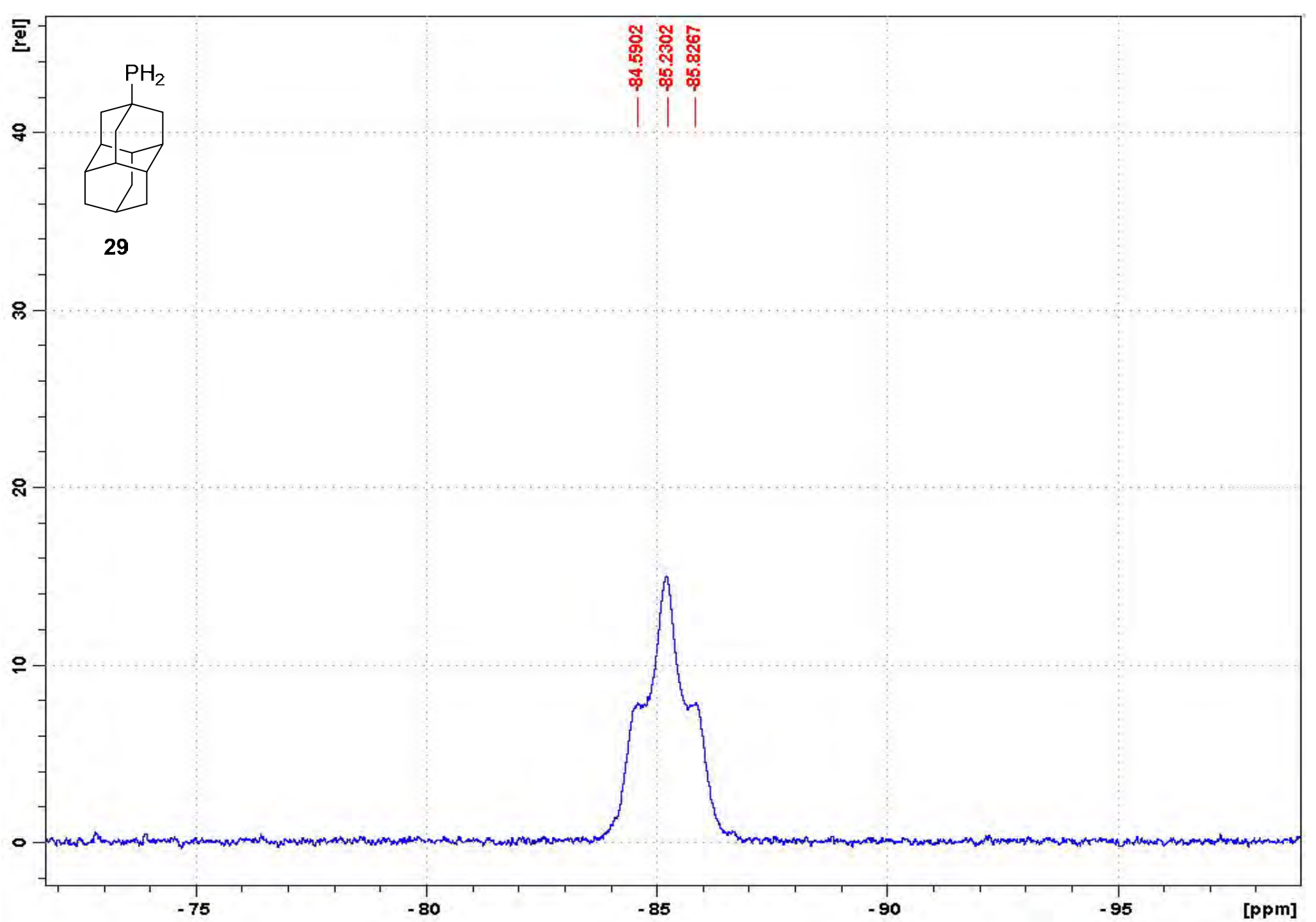


1.10.4. 4-Dia- $\mathrm{PH}_{2}{ }^{31} \mathrm{P}\left\{{ }^{1} \mathrm{H}\right\}$

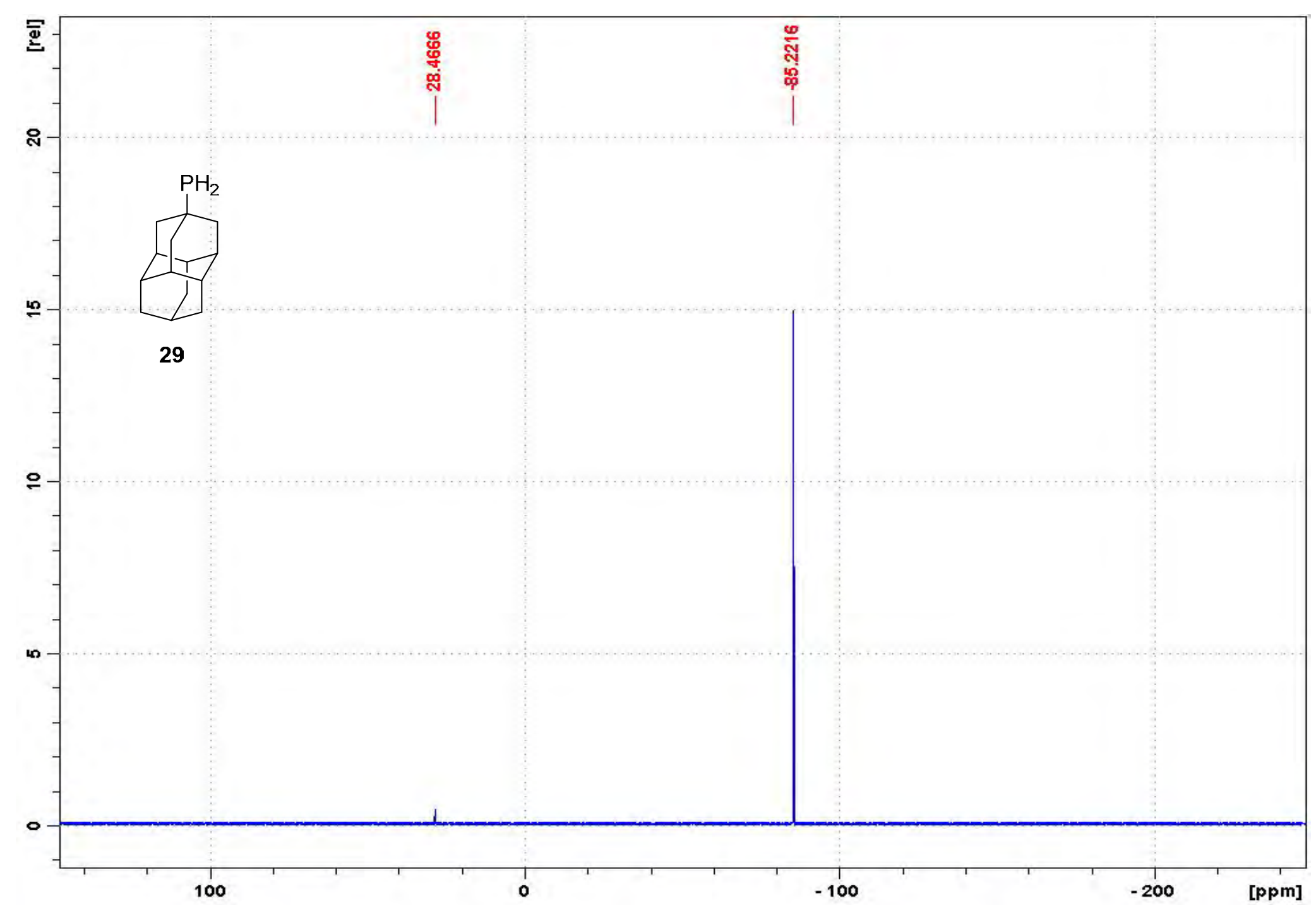


1.11. 4-Diamantyldimetylphenylphosphazo-p-nitrobenzene (30)

1.11.1. 4-Dia-PMe ${ }_{2}=\mathrm{NPhNO}_{2}{ }^{1} \mathrm{H}$

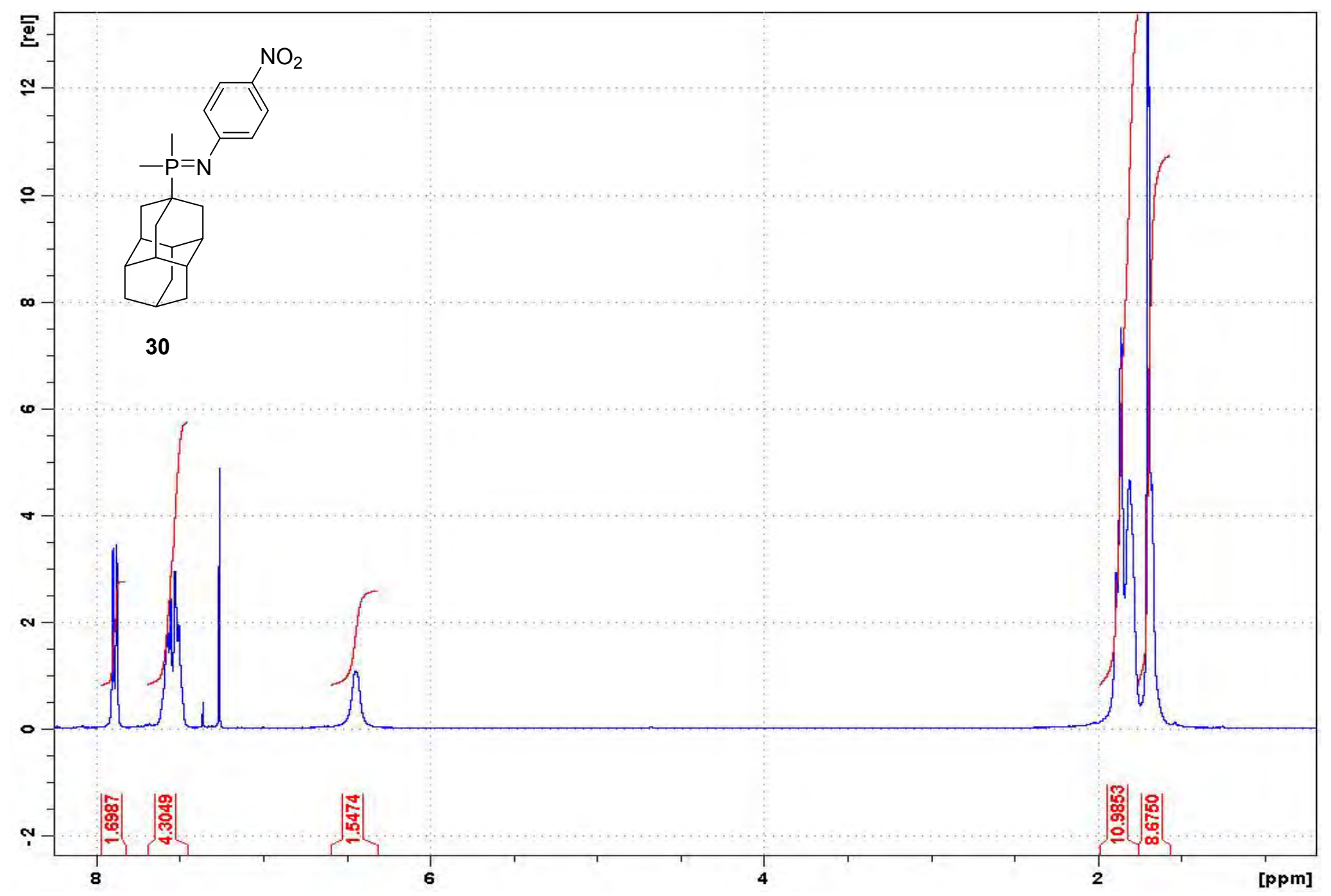


1.11.2. 4 -Dia-PMe ${ }_{2}=\mathrm{NPhNO}_{2}{ }^{13} \mathrm{C}$

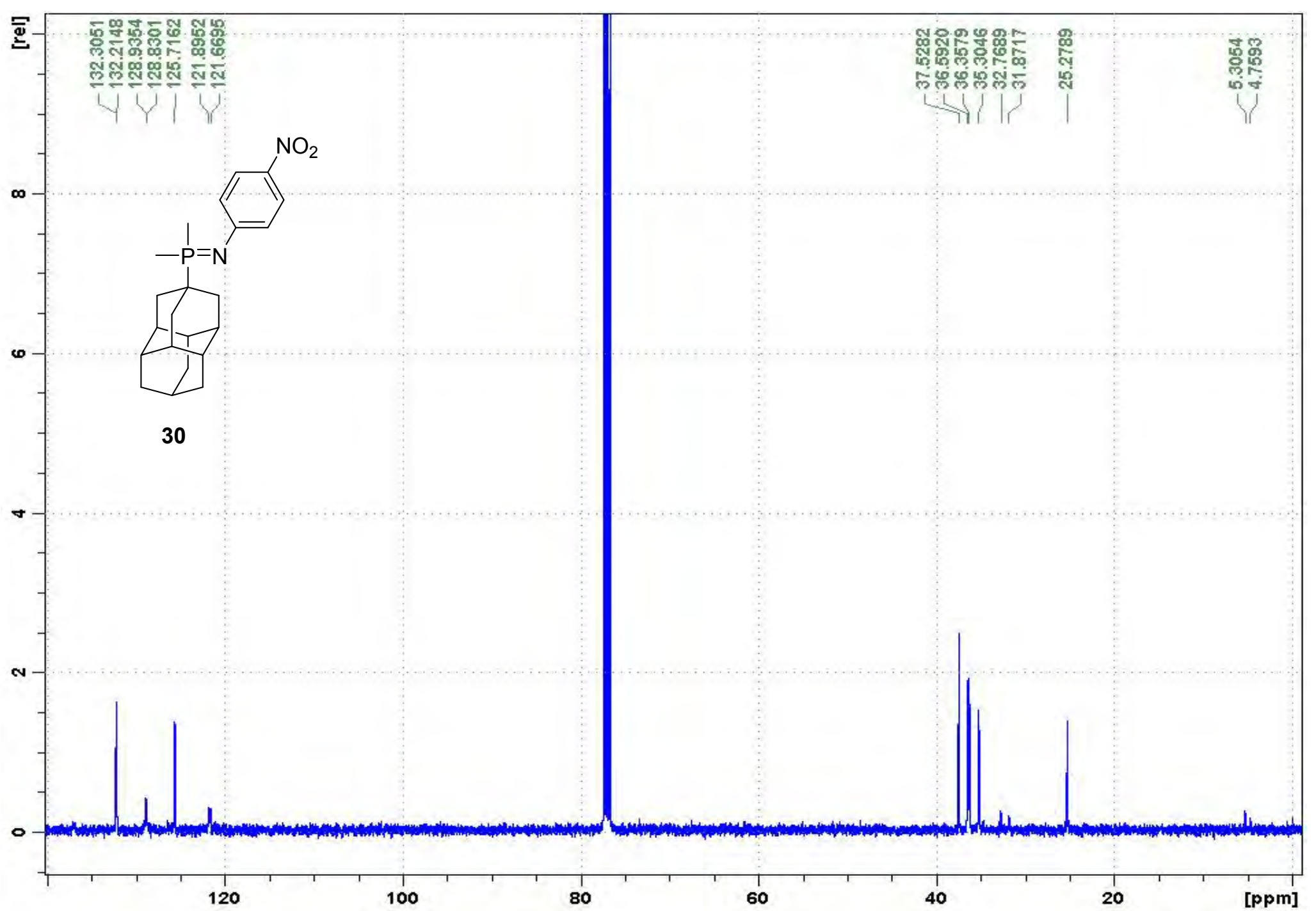


1.11.3. 4-Dia-PMe ${ }_{2}=\mathrm{NPhNO}_{2}{ }^{31} \mathrm{P}$

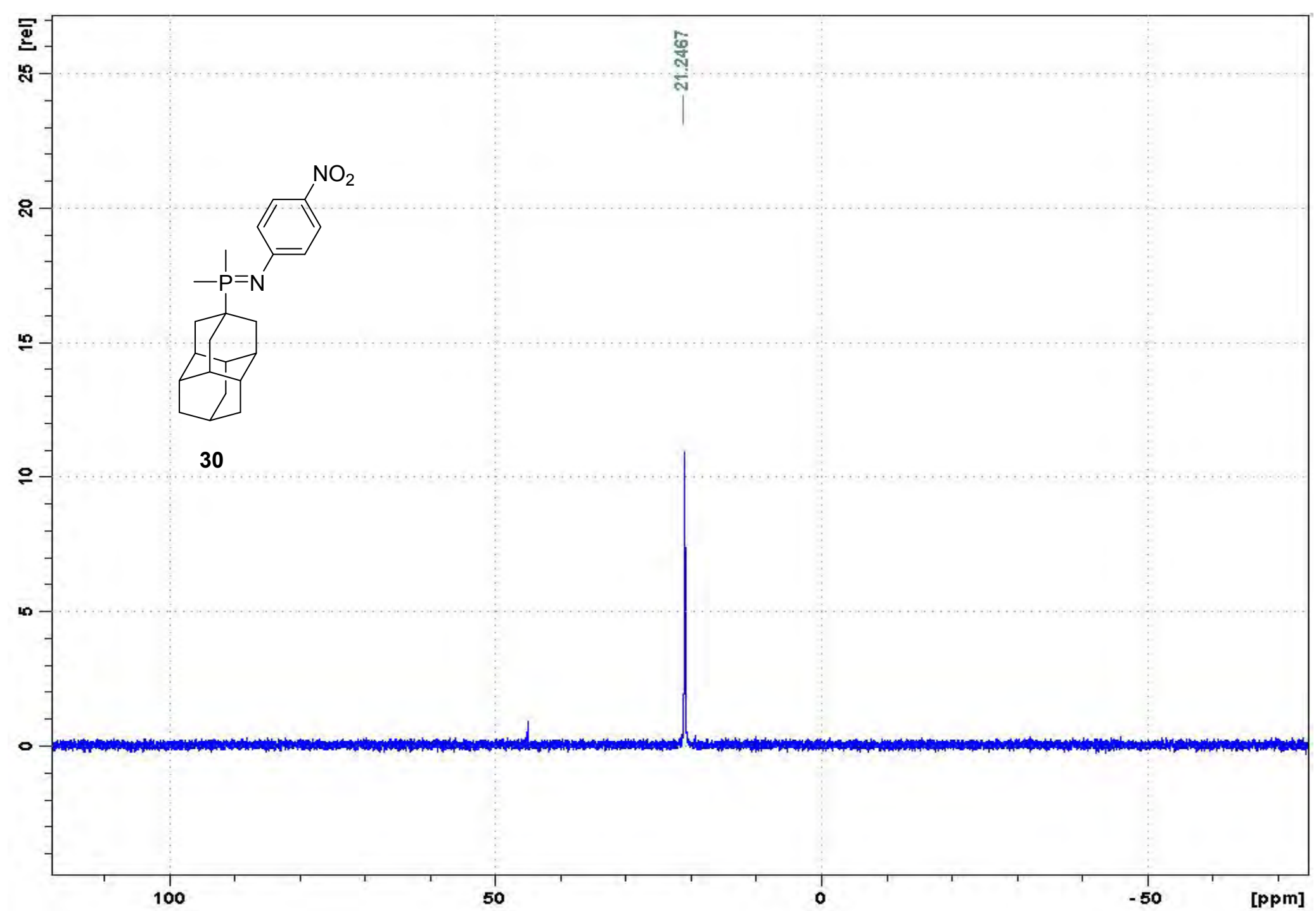


1.12. 1-Diamantylphosphine (31)

1.12.1. 1-Dia- $\mathrm{PH}_{2}{ }^{1} \mathrm{H}$

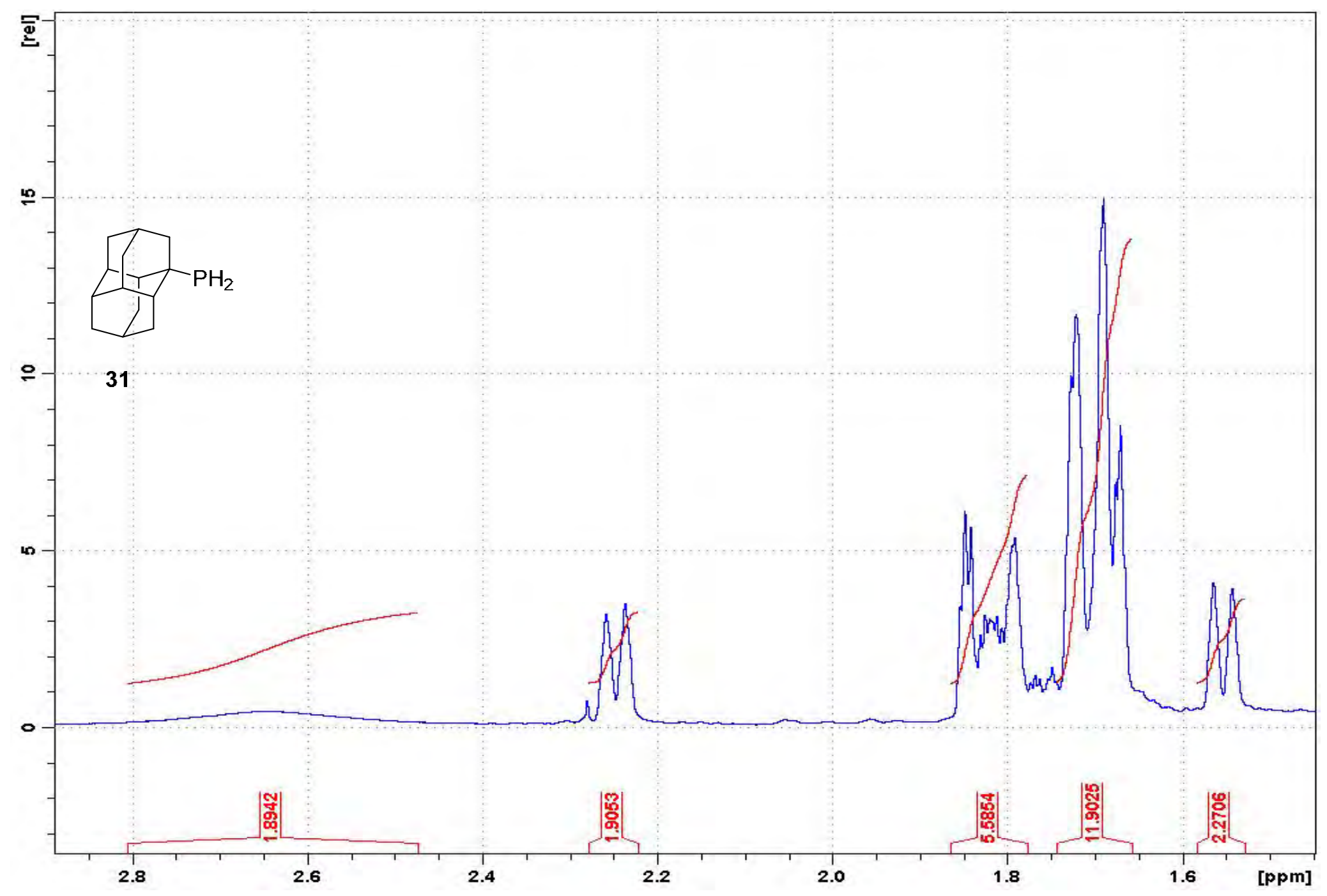


1.12.2. 1-Dia- $\mathrm{PH}_{2}{ }^{13} \mathrm{C}$

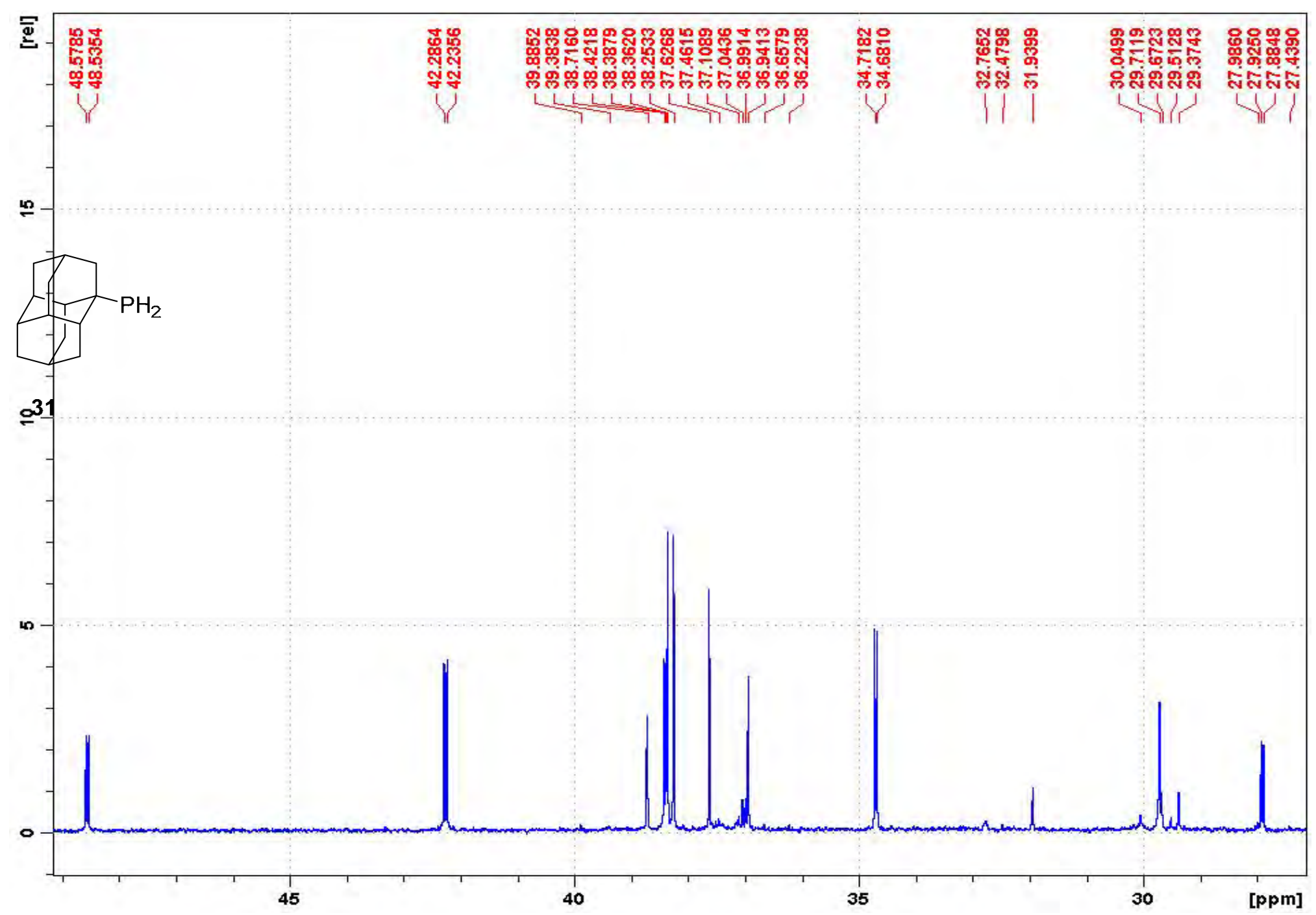


1.12.3. 1-Dia- $\mathrm{PH}_{2}{ }^{31} \mathrm{P}$

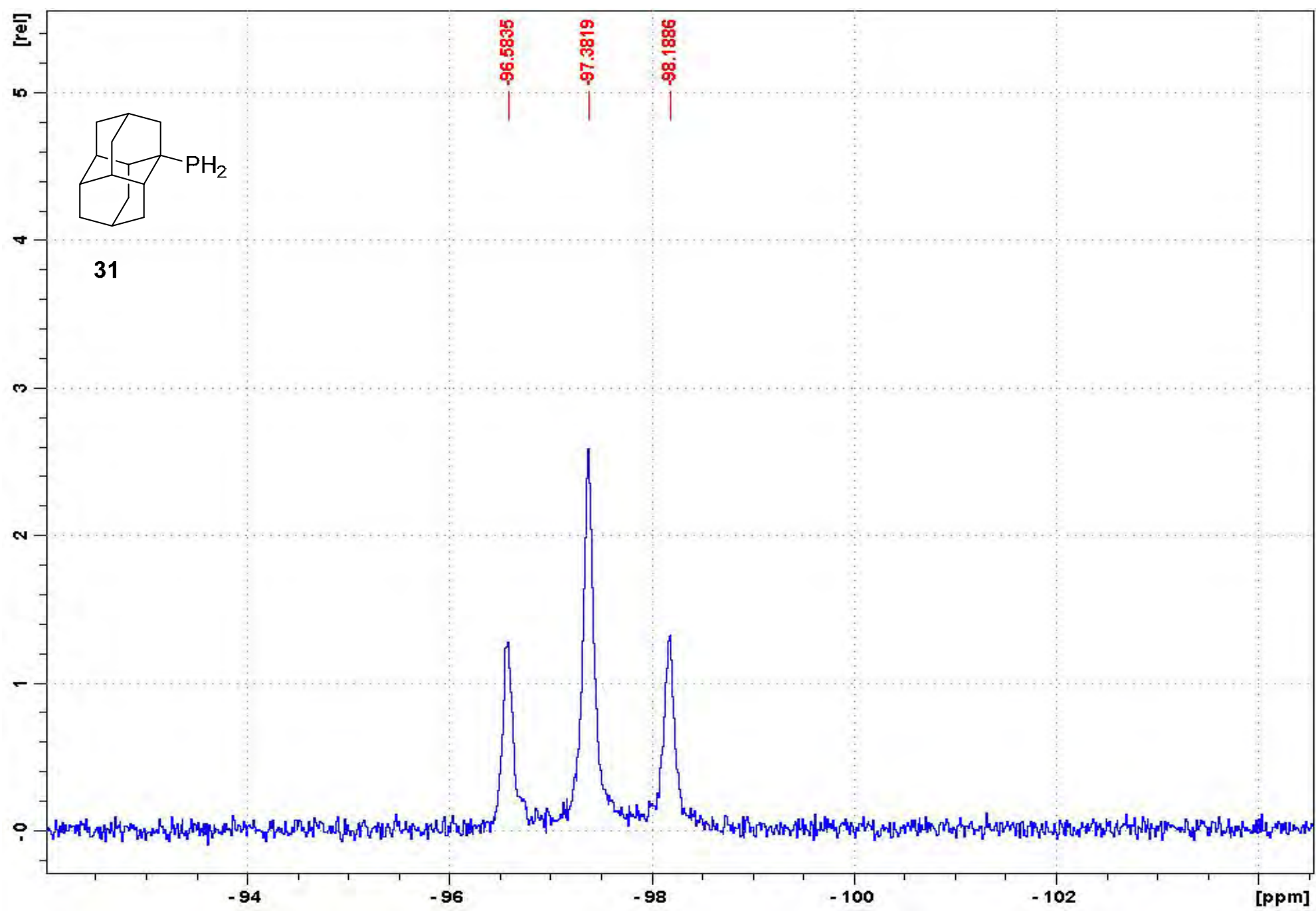


1.13. 1-Diamantyldimetylphenylphosphazo-p-nitrobenzene(32)

1.13.1. 1-Dia- $\mathrm{PMe}_{2}=\mathrm{NPhNO}_{2}{ }^{1} \mathrm{H}$

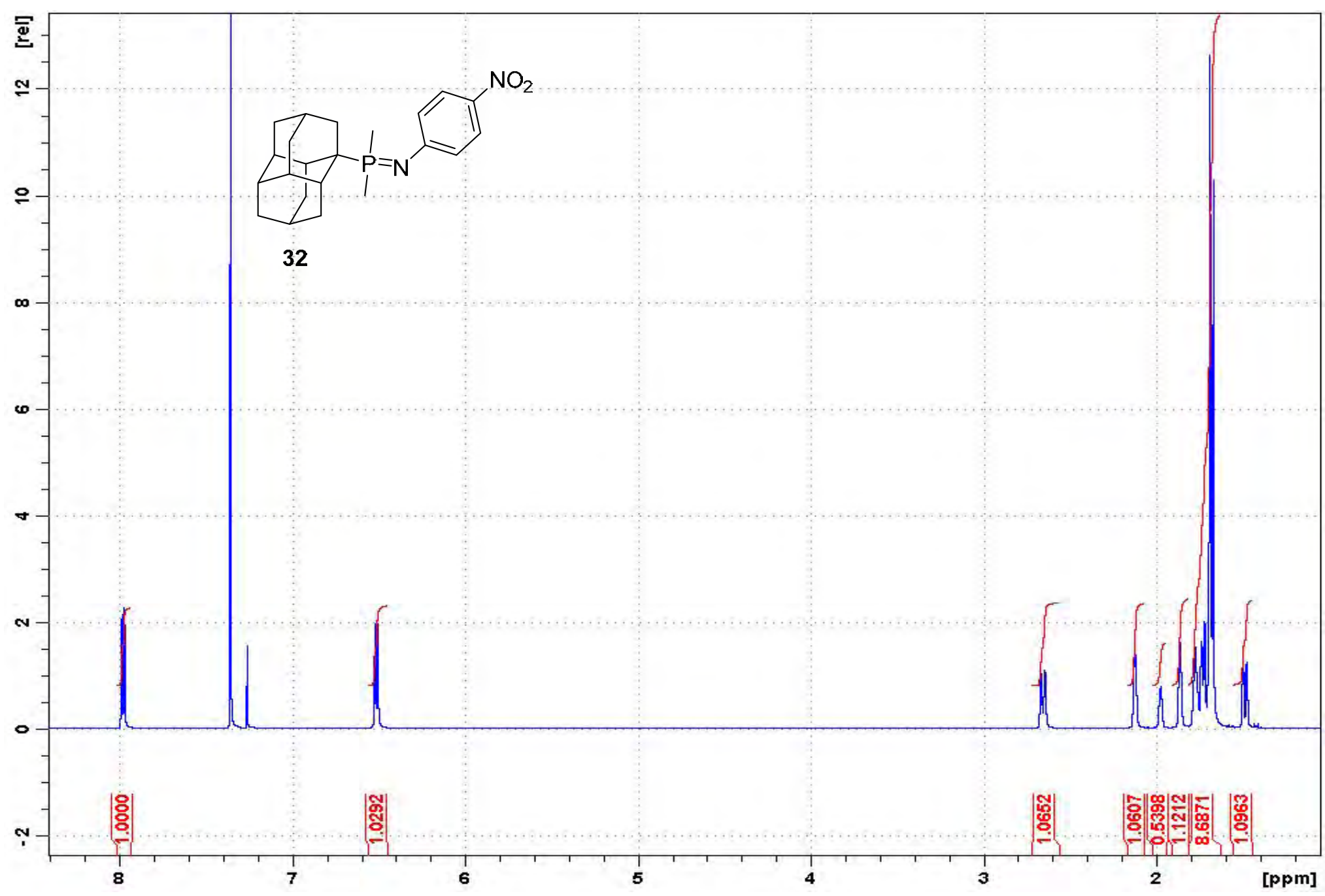


1.13.2. 1 -Dia-PMe $2=\mathrm{NPhNO}_{2}{ }^{13} \mathrm{C}$

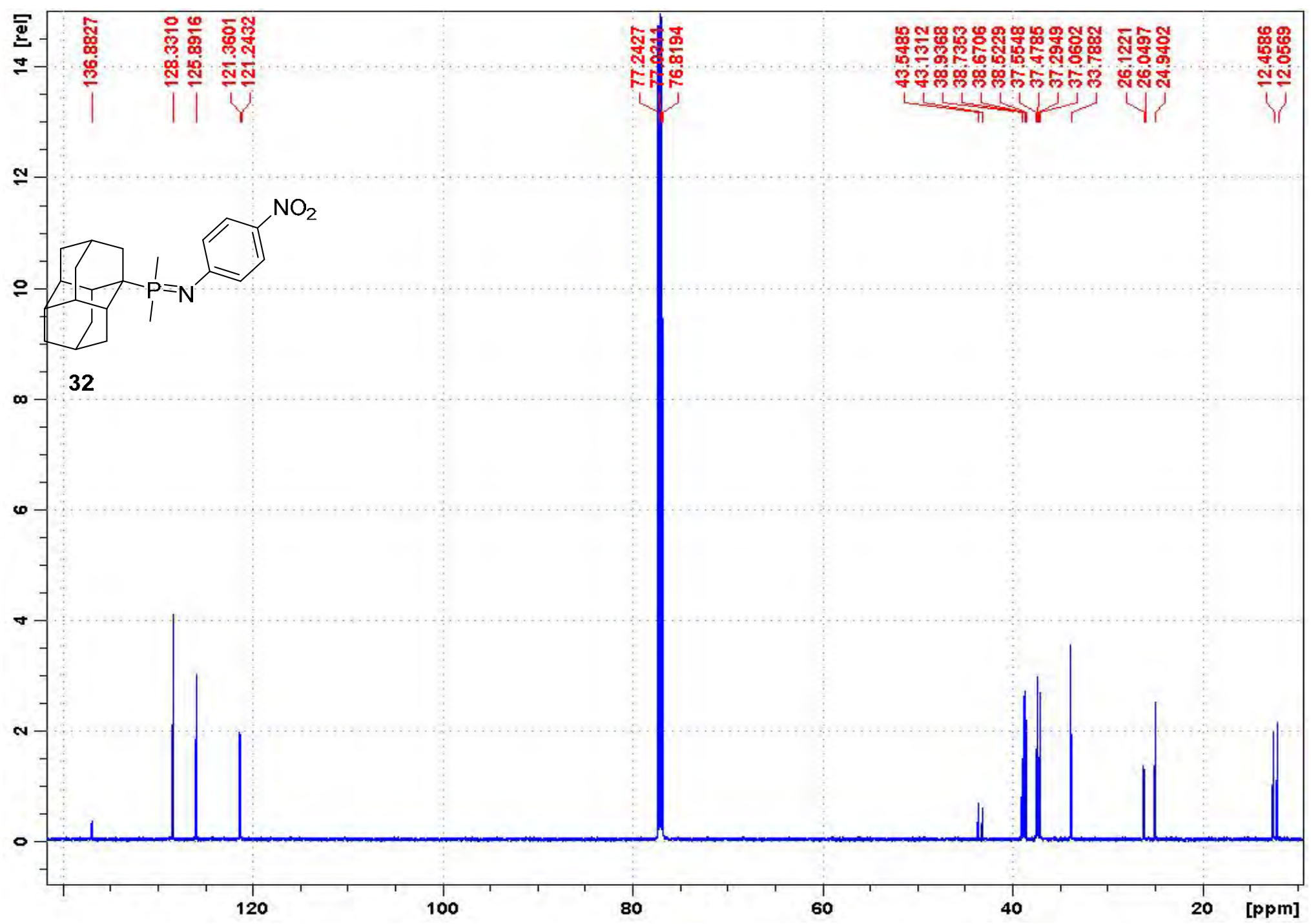


1.13.3. 1-Dia-PMe $2=\mathrm{NPhNO}_{2}{ }^{31} \mathrm{P}$

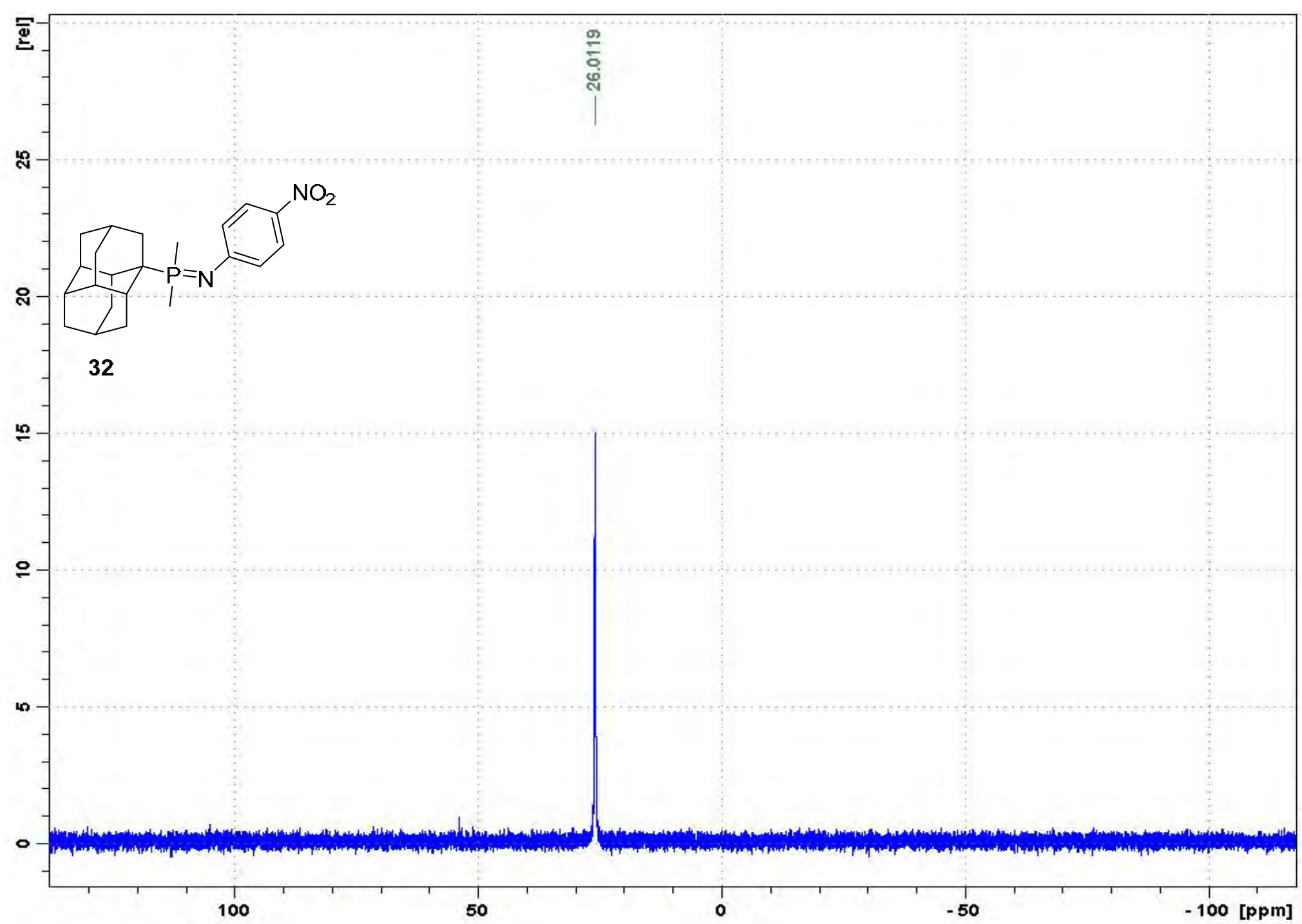


1.14. (4,9-Diamantyl)diphosphine (33)

1.14.1. 4,9-dia- $\left(\mathrm{PH}_{2}\right)_{2}{ }^{1} \mathrm{H}$

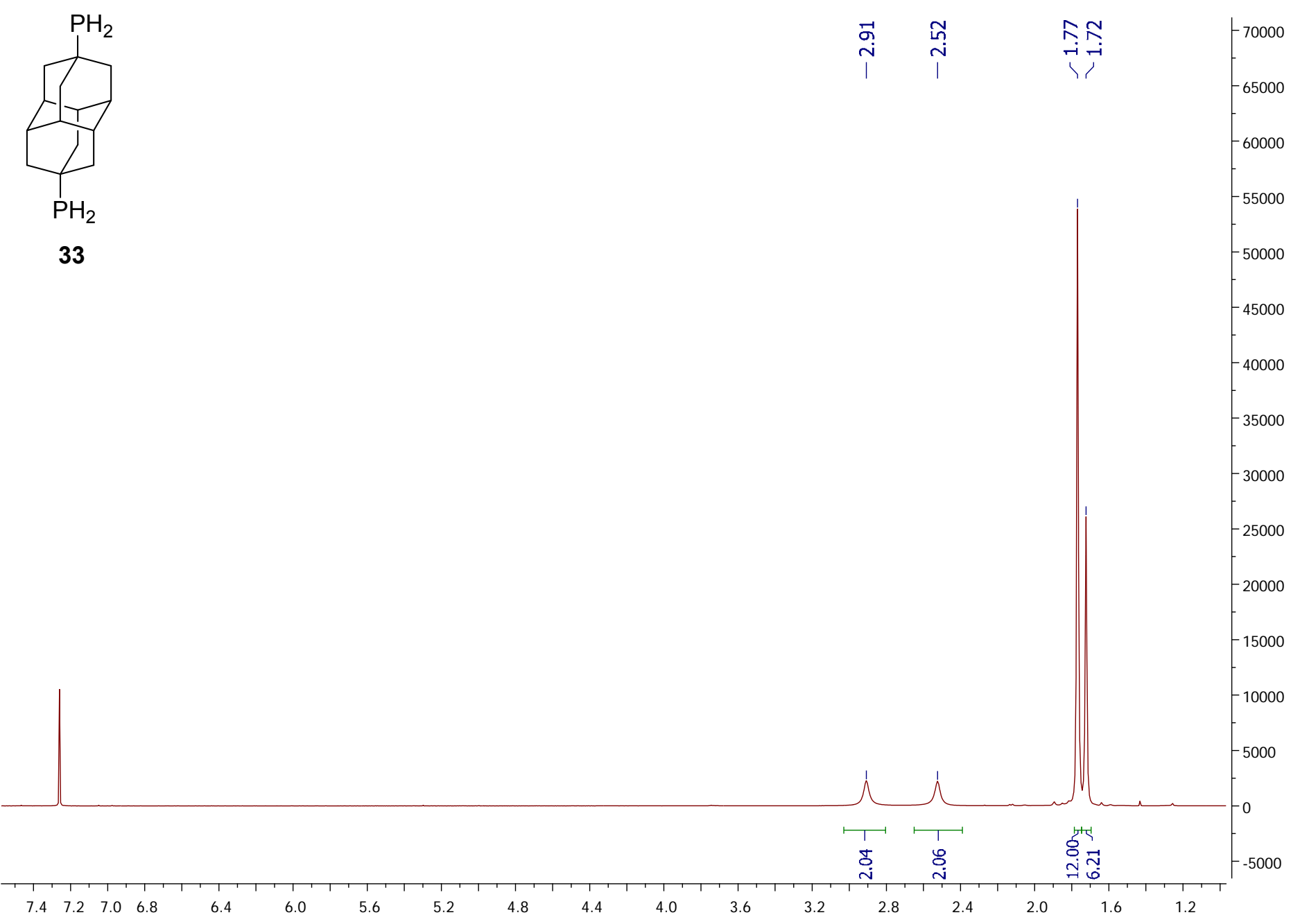


1.14.2. $4,9-$ dia- $\left(\mathrm{PH}_{2}\right)_{2}{ }^{13} \mathrm{C}$

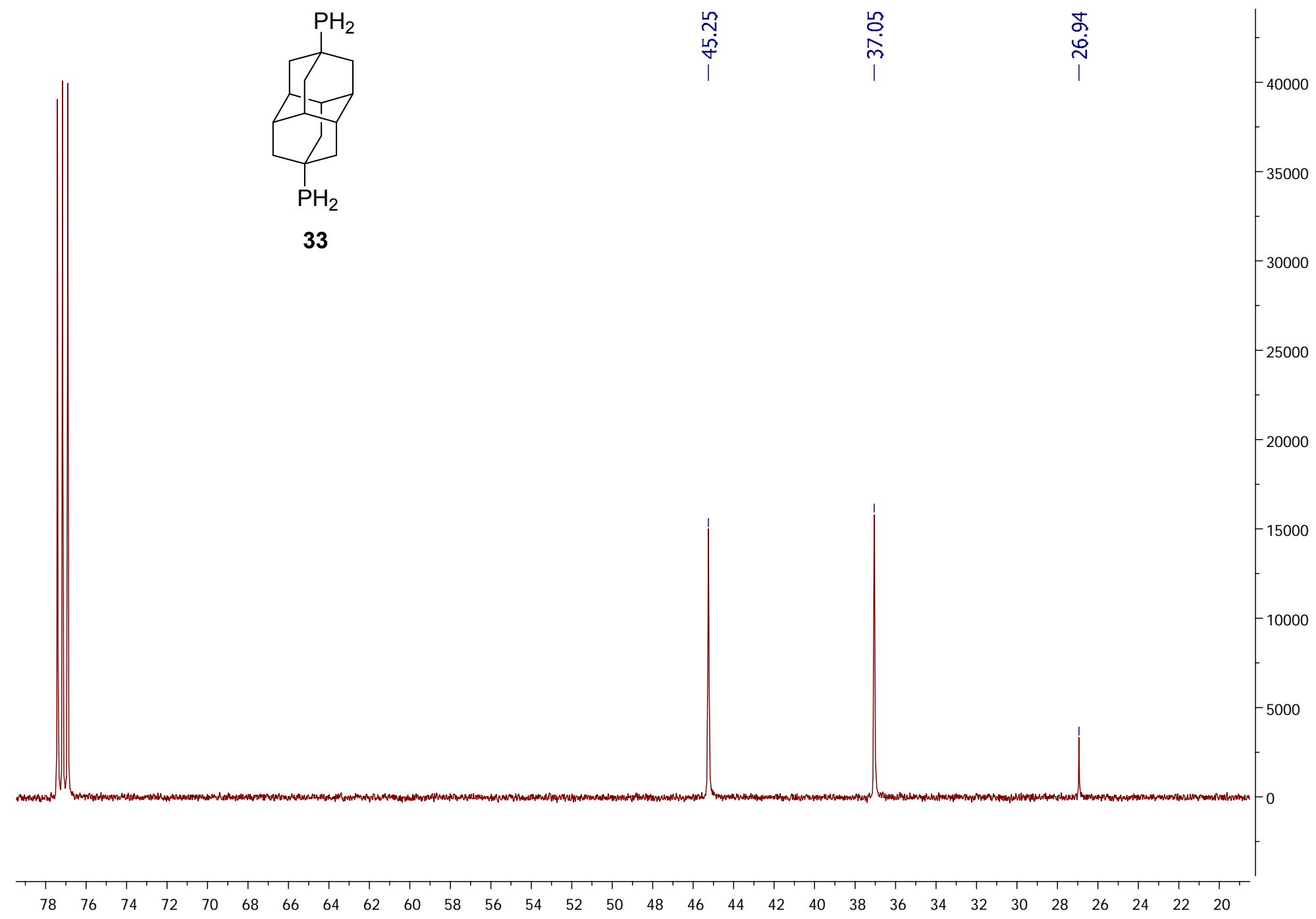


1.14.3. $4,9-$ dia- $\left(\mathrm{PH}_{2}\right)_{2}{ }^{31} \mathrm{P}$

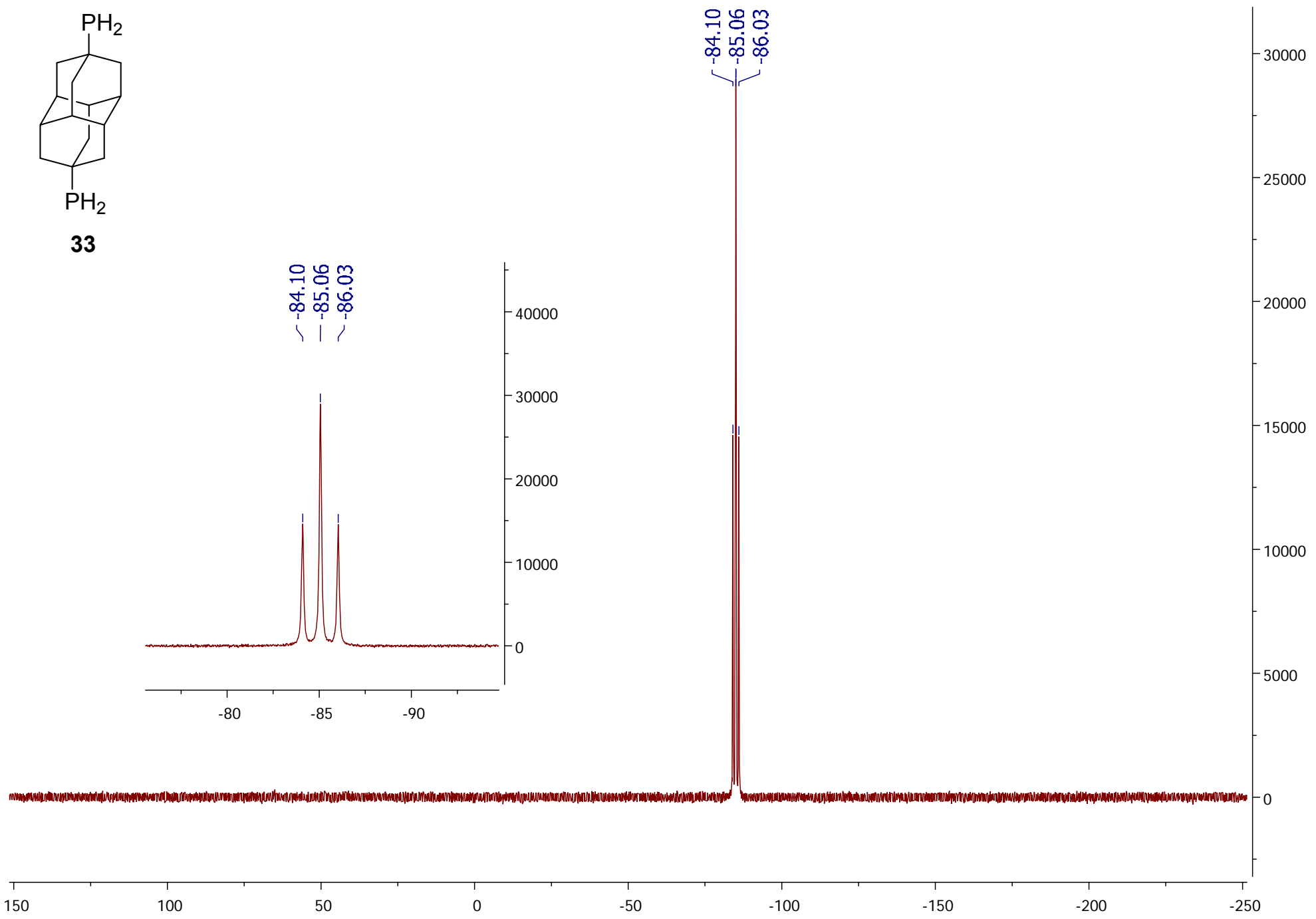


1.14.4. 4,9-dia- $\left(\mathrm{PH}_{2}\right)_{2}{ }^{31} \mathrm{P}\left\{{ }^{1} \mathrm{H}\right\}$

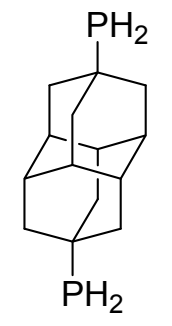

足

50000

33 
1.15. 3-Phosphinoadamant-1-ol (34)

1.15.1. 3- $\mathrm{PH}_{2}$-ada-1- $\mathrm{OH}^{1} \mathrm{H}$

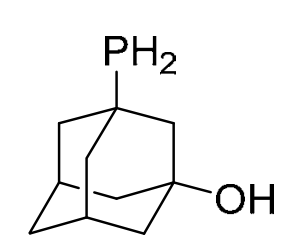

34

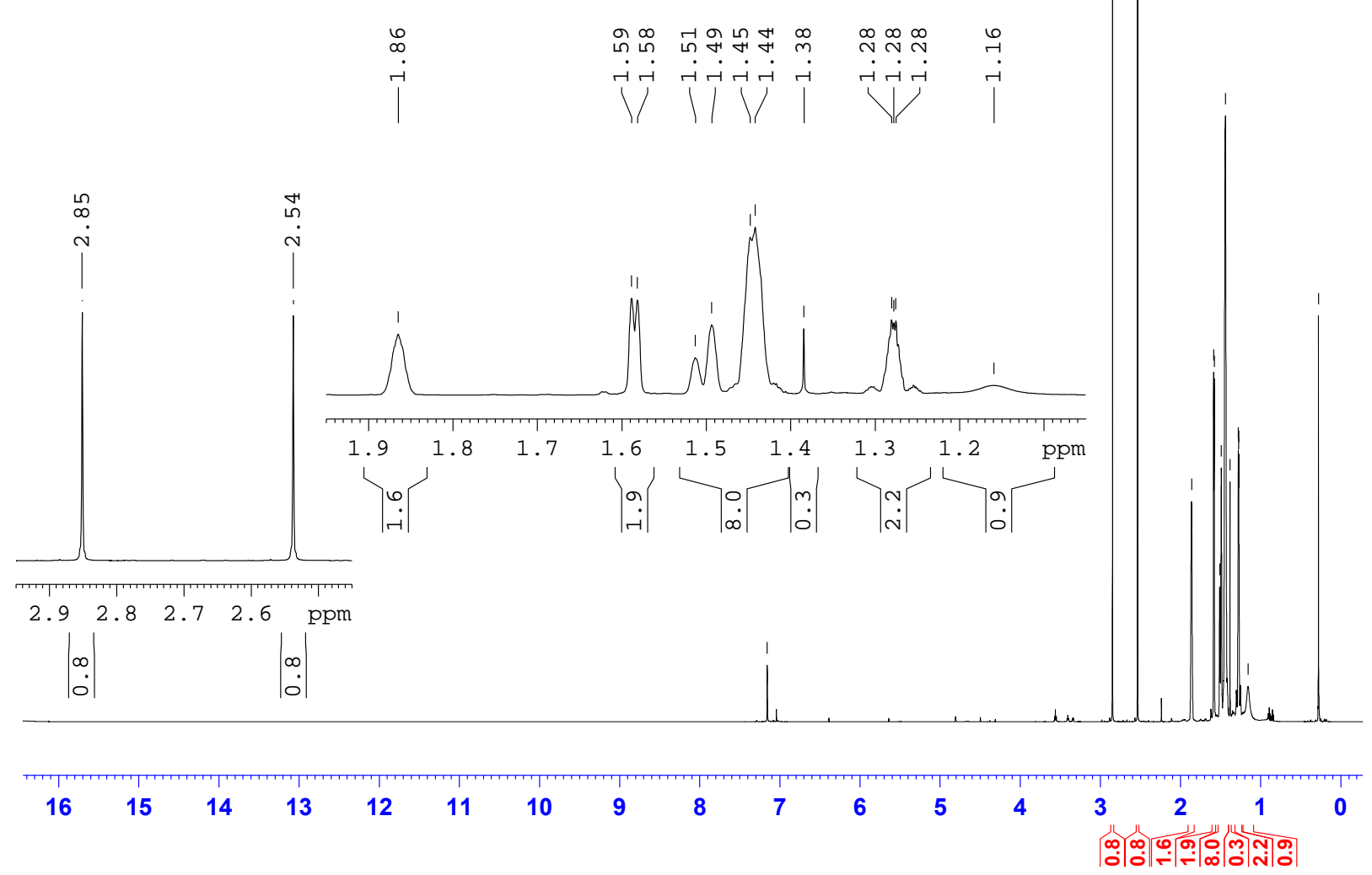


1.15.2. $3-\mathrm{PH}_{2}$-ada-1-OH ${ }^{13} \mathrm{C}$

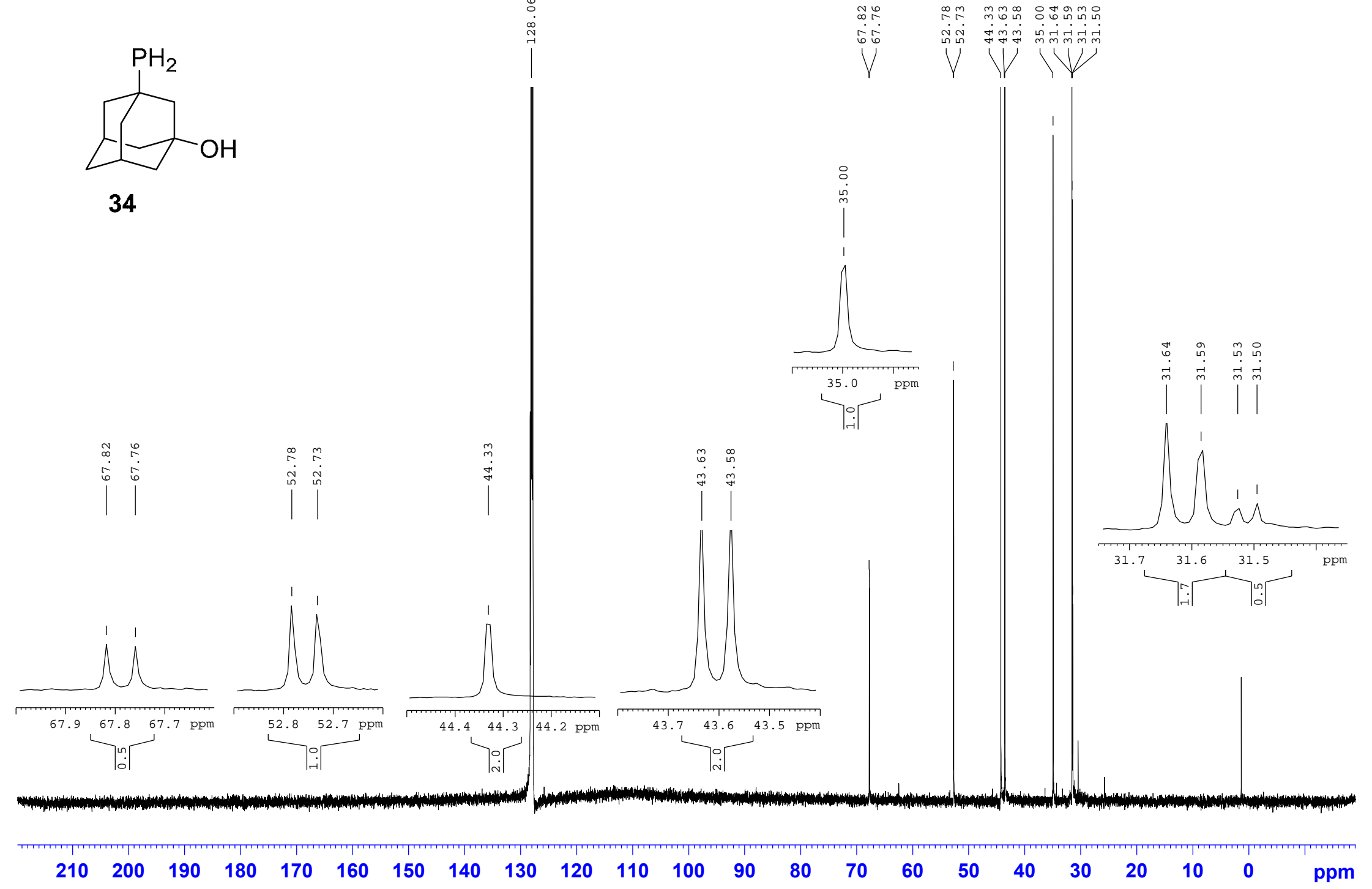


1.15.3. $3-\mathrm{PH}_{2}$-ada-1-OH ${ }^{31} \mathrm{P}$

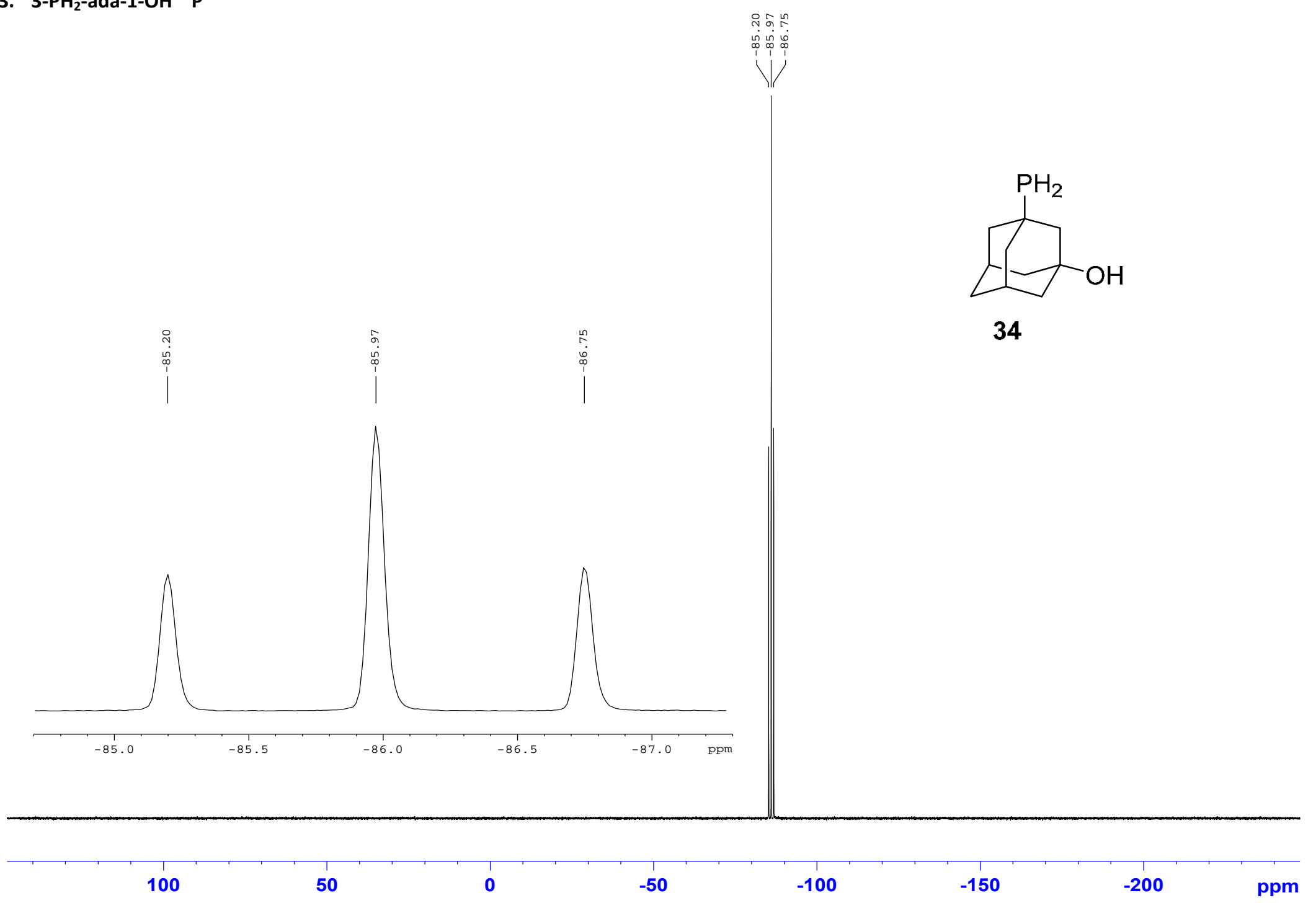


1.15.4. 3- $\mathrm{PH}_{2}$-ada-1-OH ${ }^{31} \mathrm{P}\left\{{ }^{1} \mathrm{H}\right\}$

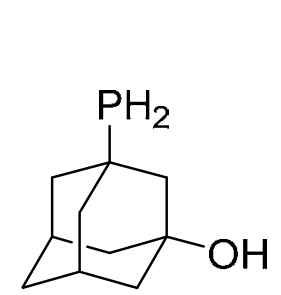

34

100

50

0

$-50$

$-100$

$-150$

$-200$

ppm 
1.16. (3-Hydroxydamantan-1-yl)phosphine oxide (35)

1.16.1. 3-HO-ada-1-P( $=\mathrm{O}) \mathrm{H}_{2}{ }^{1} \mathrm{H}$

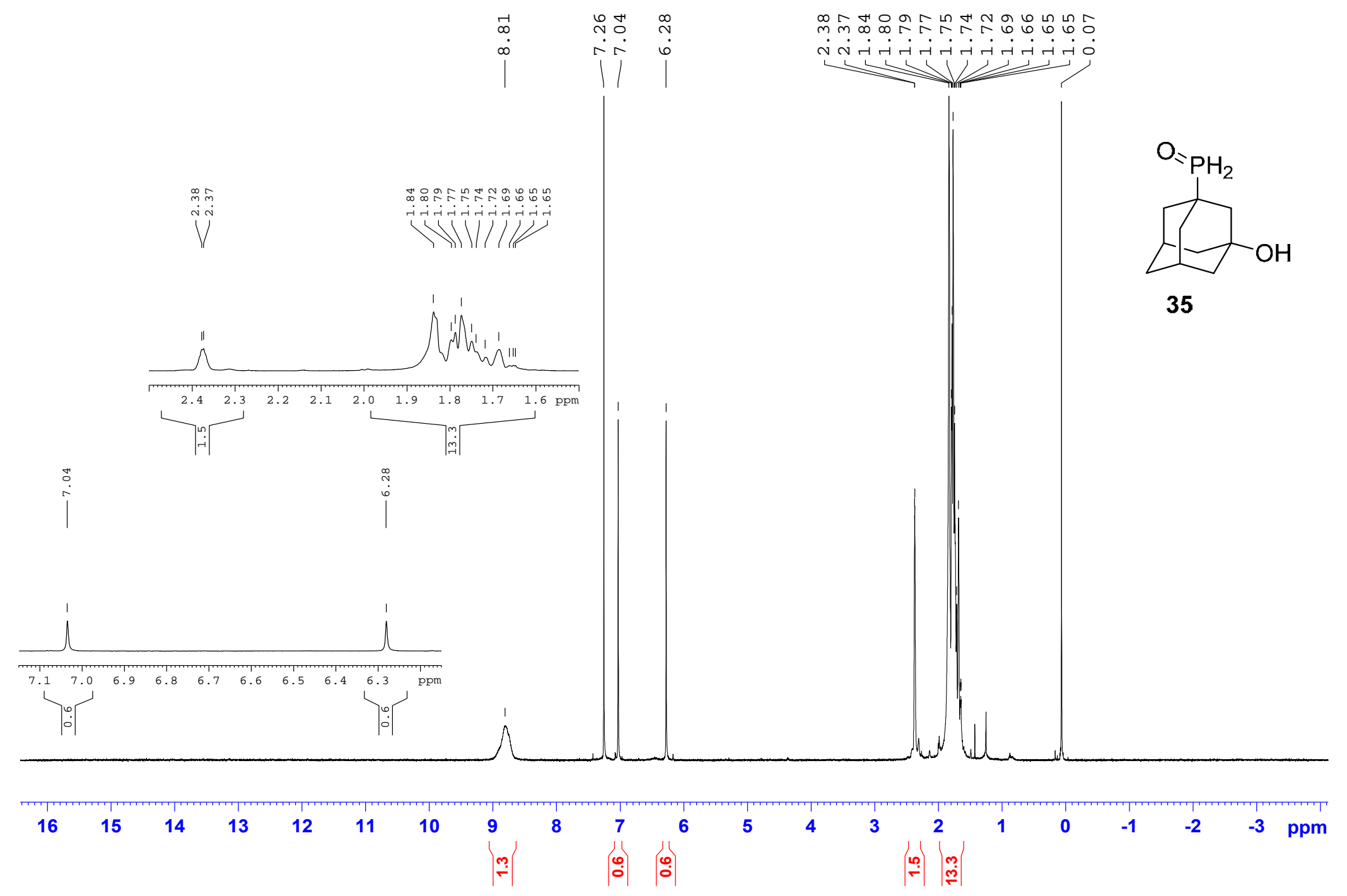


1.16.2. $3-\mathrm{HO}$-ada-1-P(=O) $\mathrm{H}_{2}{ }^{13} \mathrm{C}$

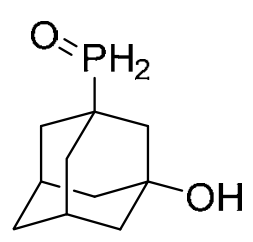

35

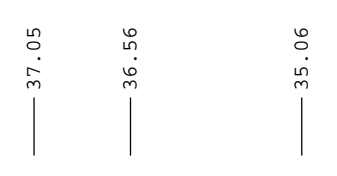

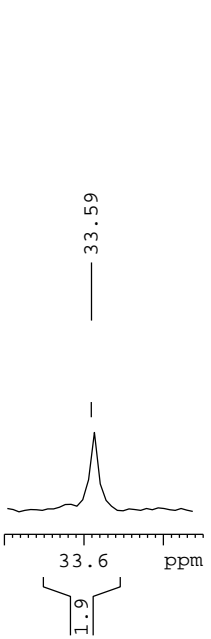

$$
\begin{aligned}
& \begin{array}{ll}
0 & 0 \\
\stackrel{0}{i} & 0 \\
0 & 0 \\
&
\end{array}
\end{aligned}
$$

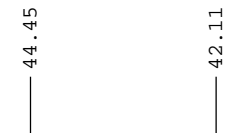

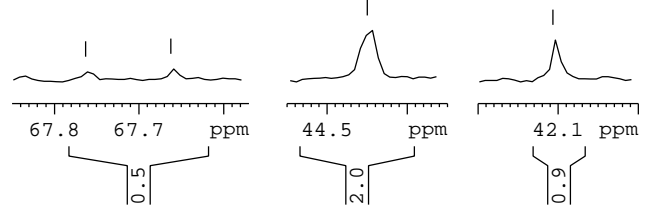

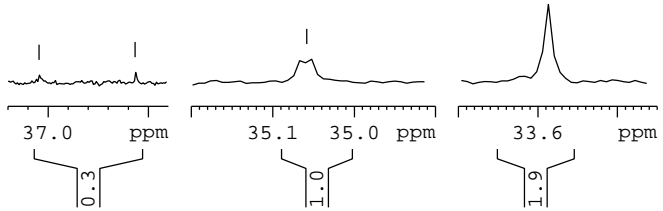

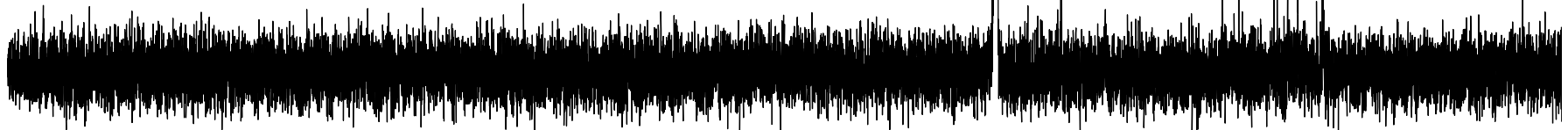


1.16.3. $3-\mathrm{HO}-$ ada-1- $\mathrm{P}(=\mathrm{O}) \mathrm{H}_{2}{ }^{31} \mathrm{P}$

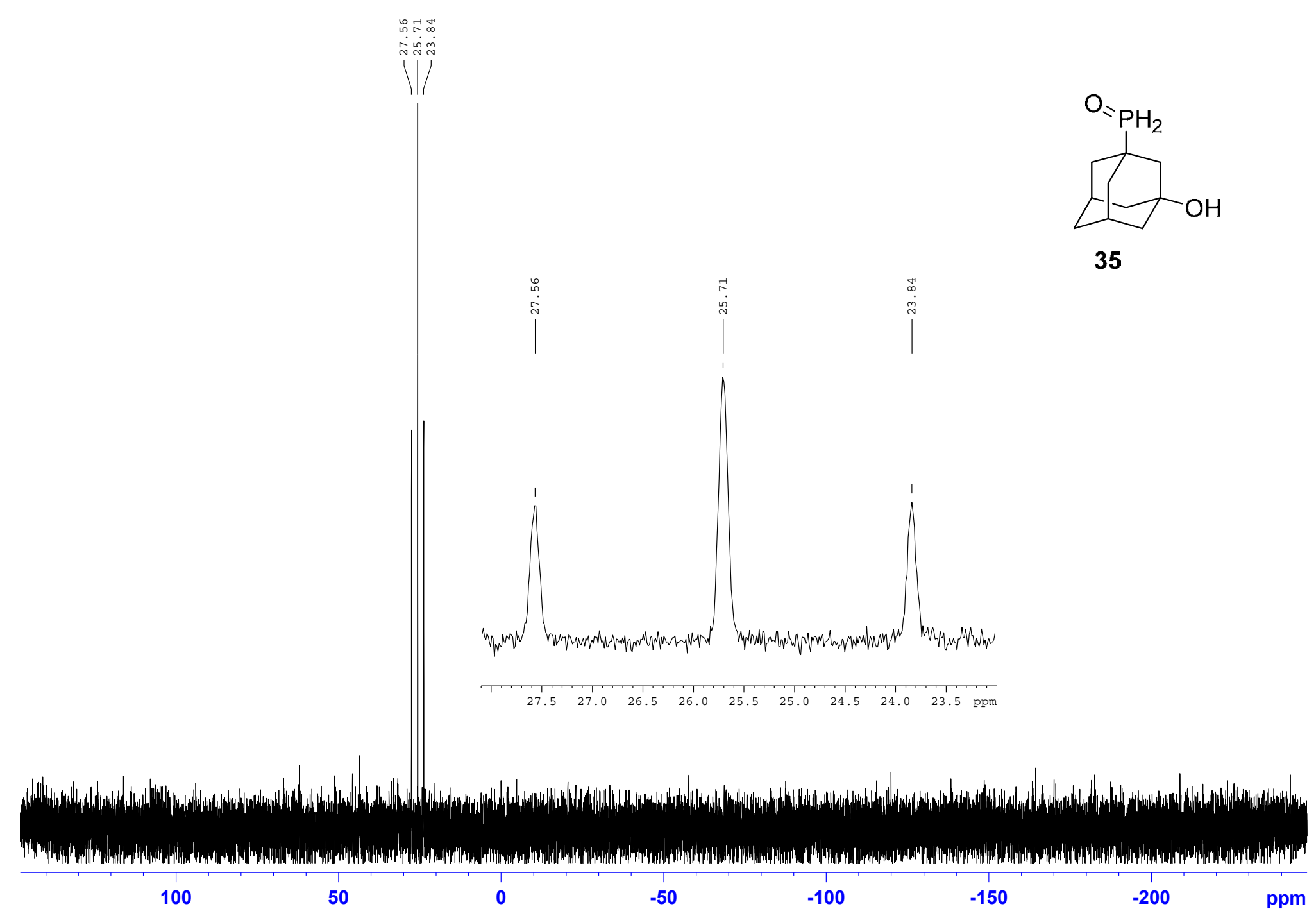


1.16.4. 3-HO-ada-1-P(=O) $\mathrm{H}_{2}{ }^{31} \mathrm{P}\left\{{ }^{1} \mathrm{H}\right\}$

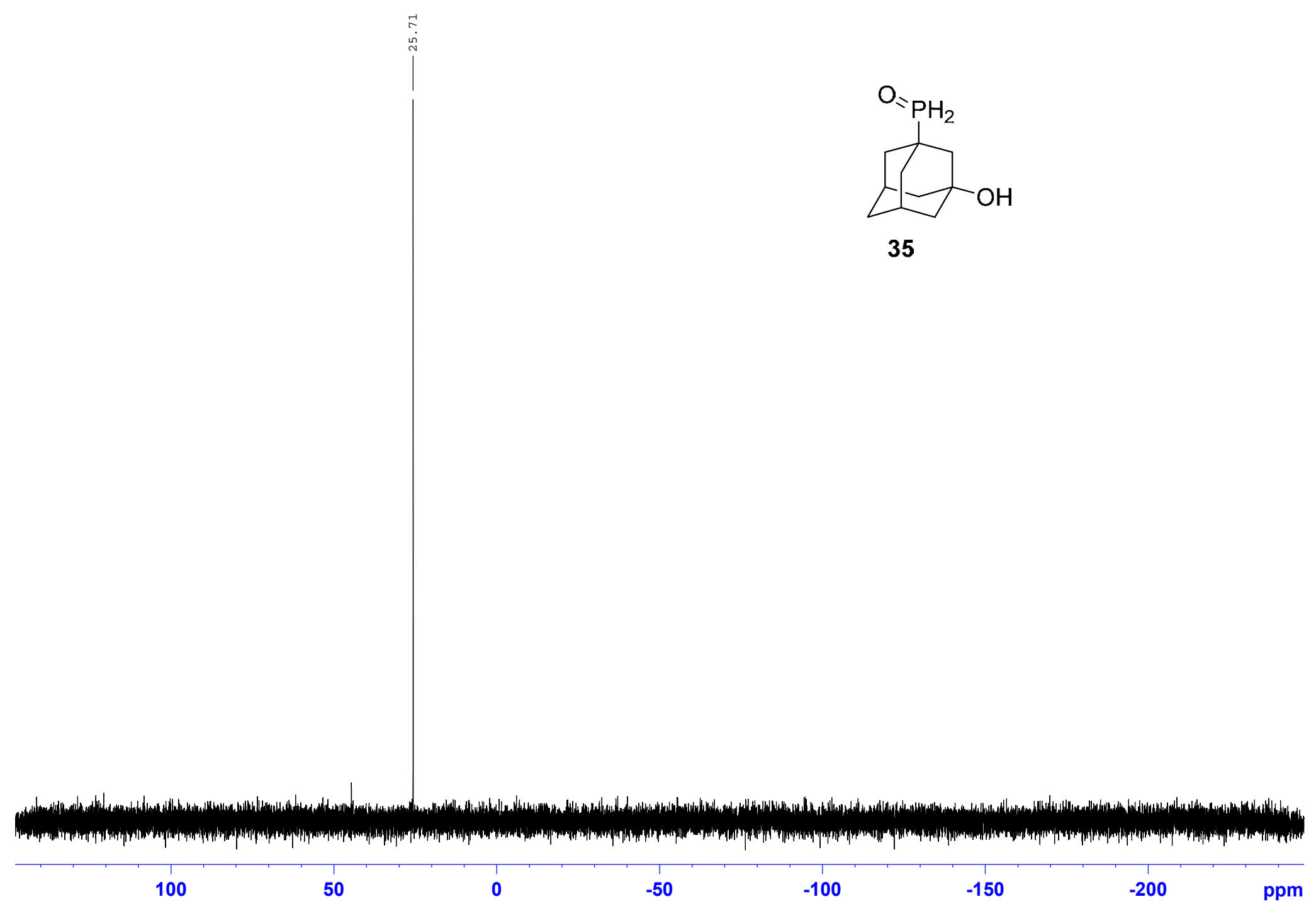


1.17. 9-Phosphinodiamantan-4-ol (36)

1.17.1. 9- $\mathrm{PH}_{2}$-dia-4- $\mathrm{OH}{ }^{1} \mathrm{H}$

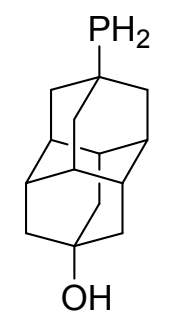

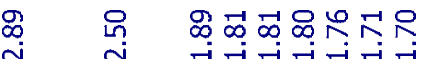

i $\quad$ i

36

我

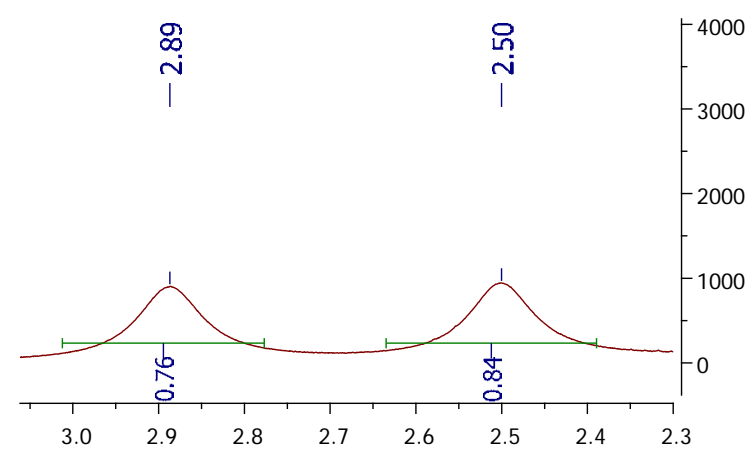

000
000
000

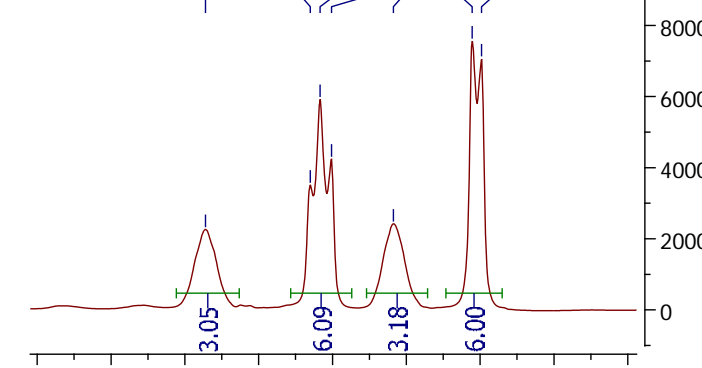

$\begin{array}{lllllllll}2.00 & 1.95 & 1.90 & 1.85 & 1.80 & 1.75 & 1.70 & 1.65 & 1.60\end{array}$

$-90000$

$-80000$

$-70000$

60000

50000

$-40000$

$-30000$

20000

$-10000$

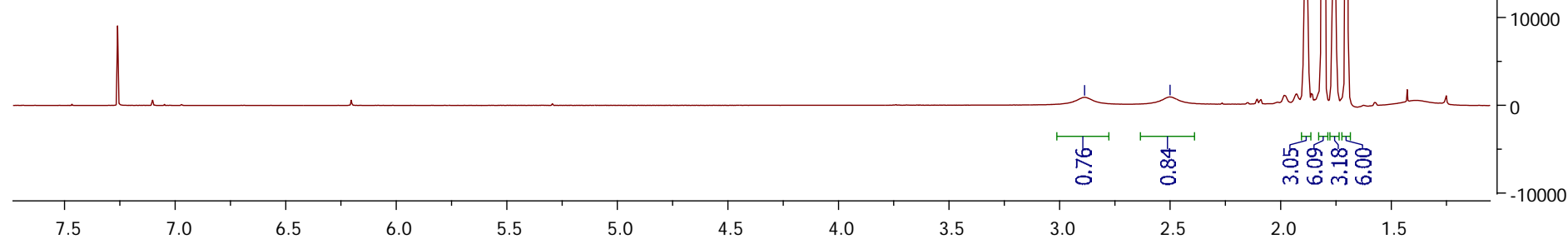


1.17.2. 9- $\mathrm{PH}_{2}-$ dia- $4-\mathrm{OH}^{13} \mathrm{C}$

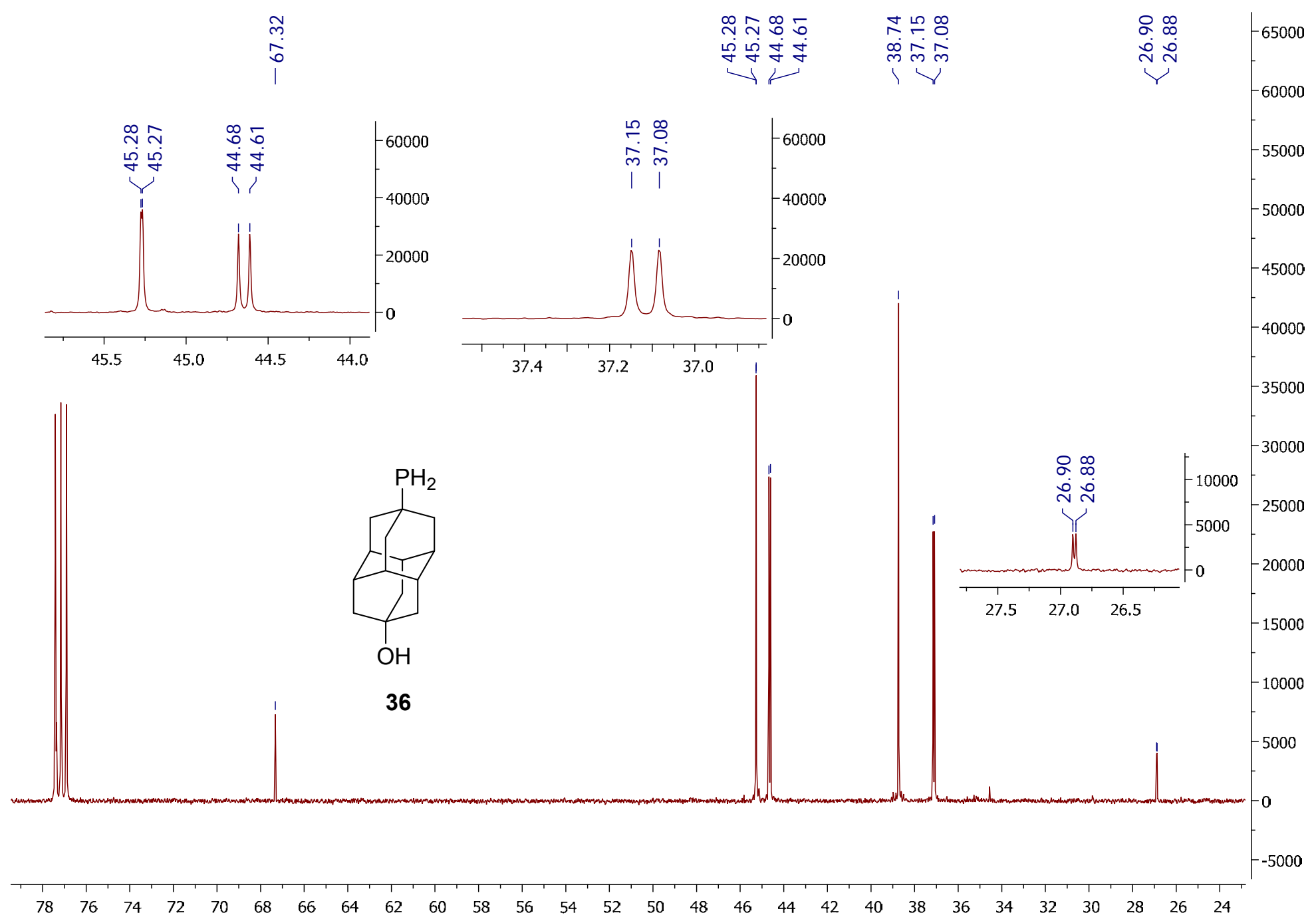


1.17.3. $9-\mathrm{PH}_{2}-$ dia- $4-O \mathrm{H}^{31} \mathrm{P}$

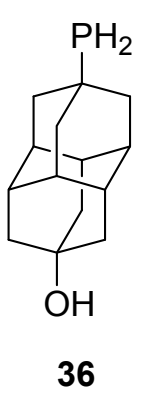

춍

क्ष

$-90000$

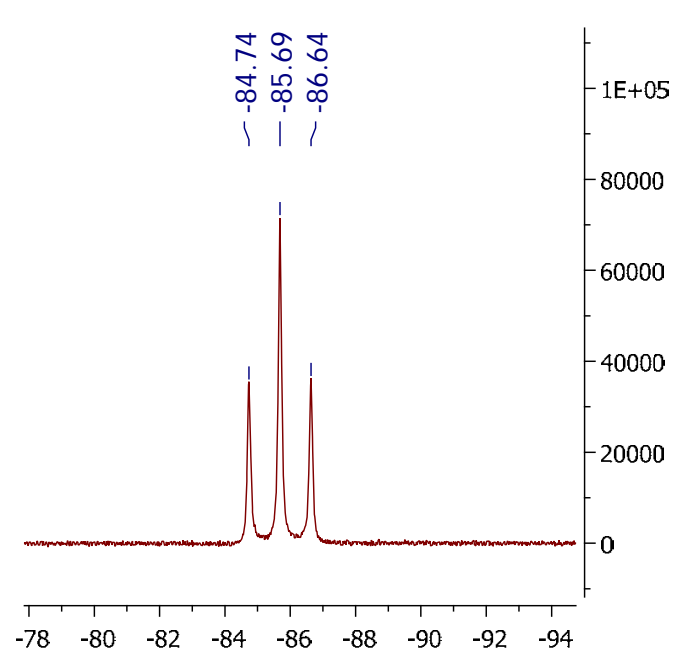


1.17.4. 9- $\mathrm{PH}_{2}$-dia-4-OH ${ }^{31} \mathrm{P}\left\{{ }^{1} \mathrm{H}\right\}$

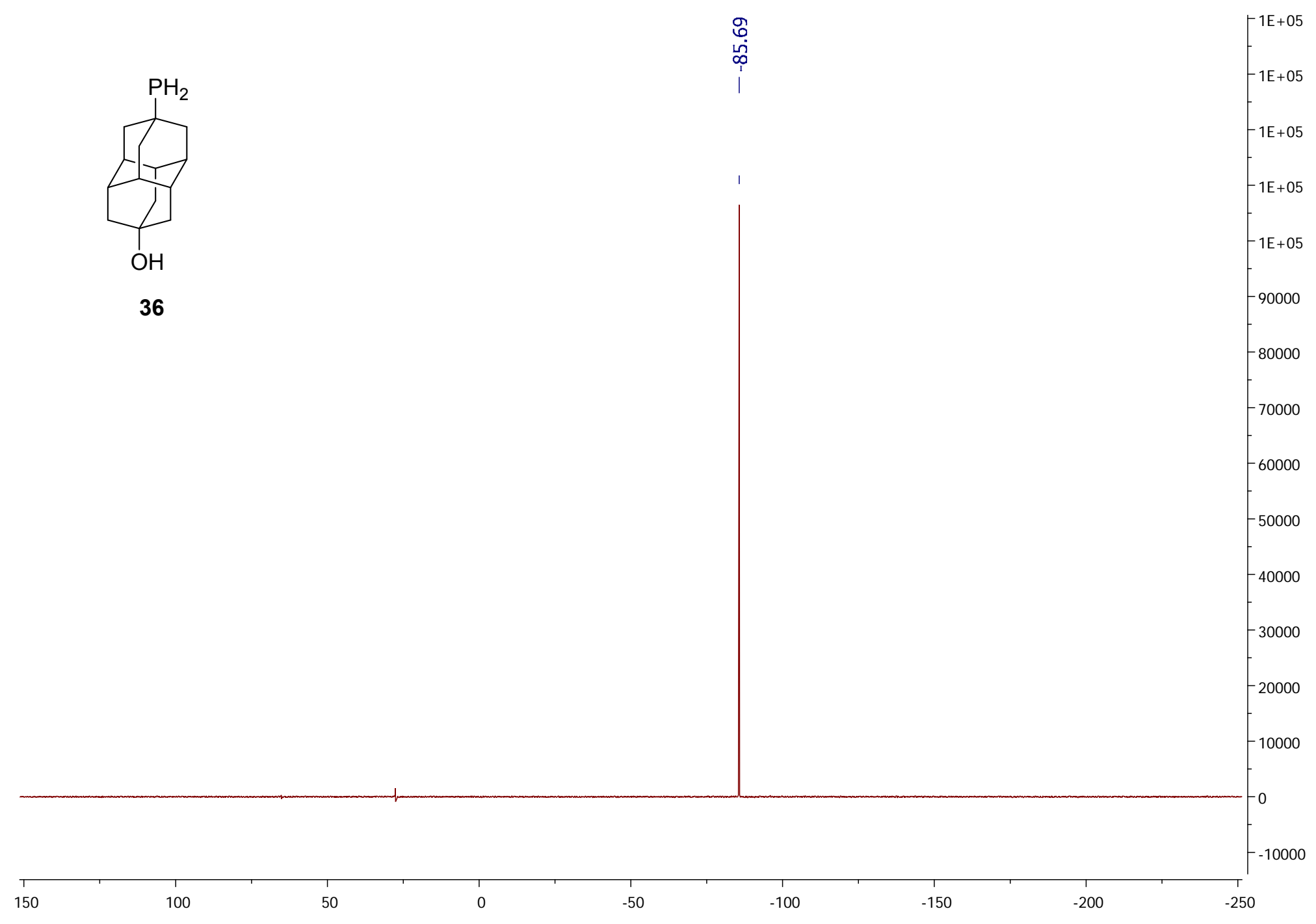


1.18. (9-Chlorodiamant-4-yl) phosphine (37)

1.18.1. 9-Cl-dia-4- $\mathrm{PH}_{2}{ }^{1} \mathrm{H}$

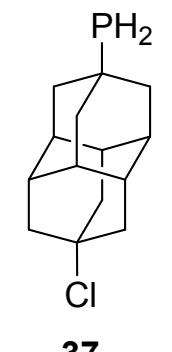

37

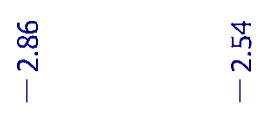

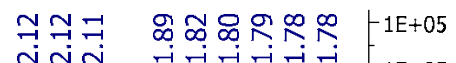

ن

$-90000$

80000

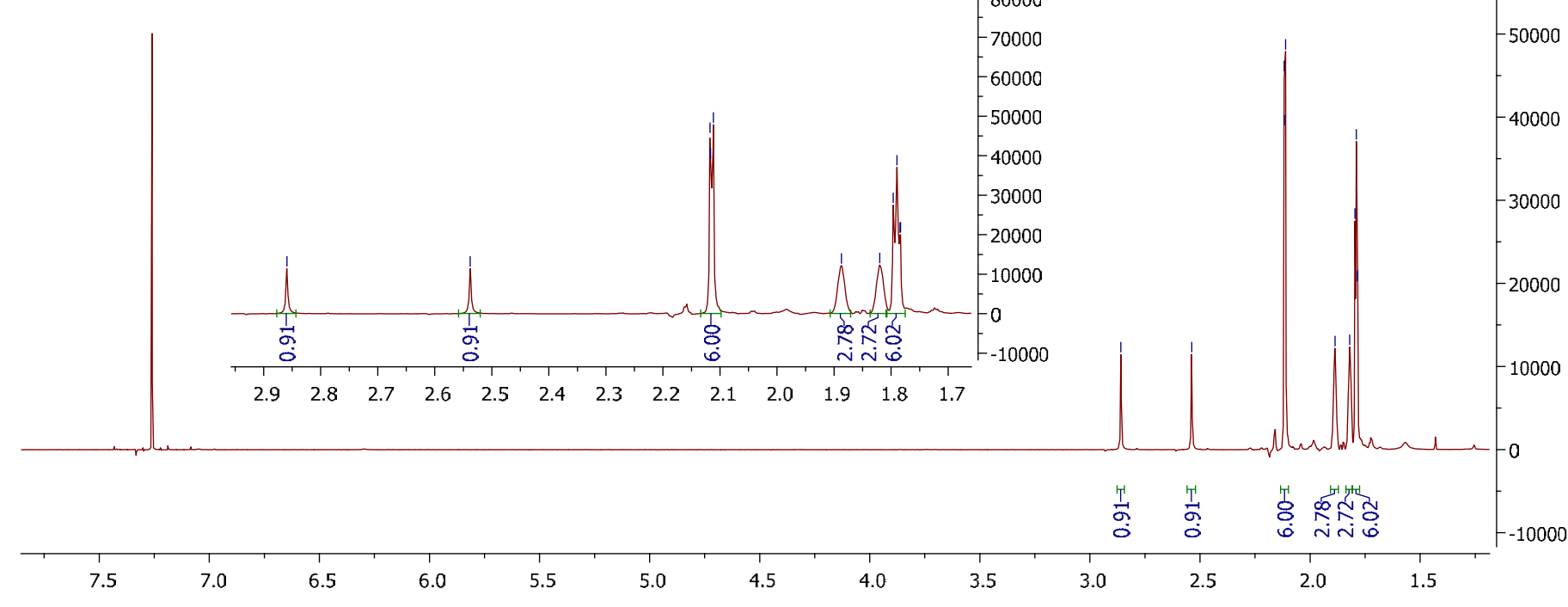


1.18.2. 9-Cl-dia-4- $\mathrm{PH}_{2}{ }^{13} \mathrm{C}$

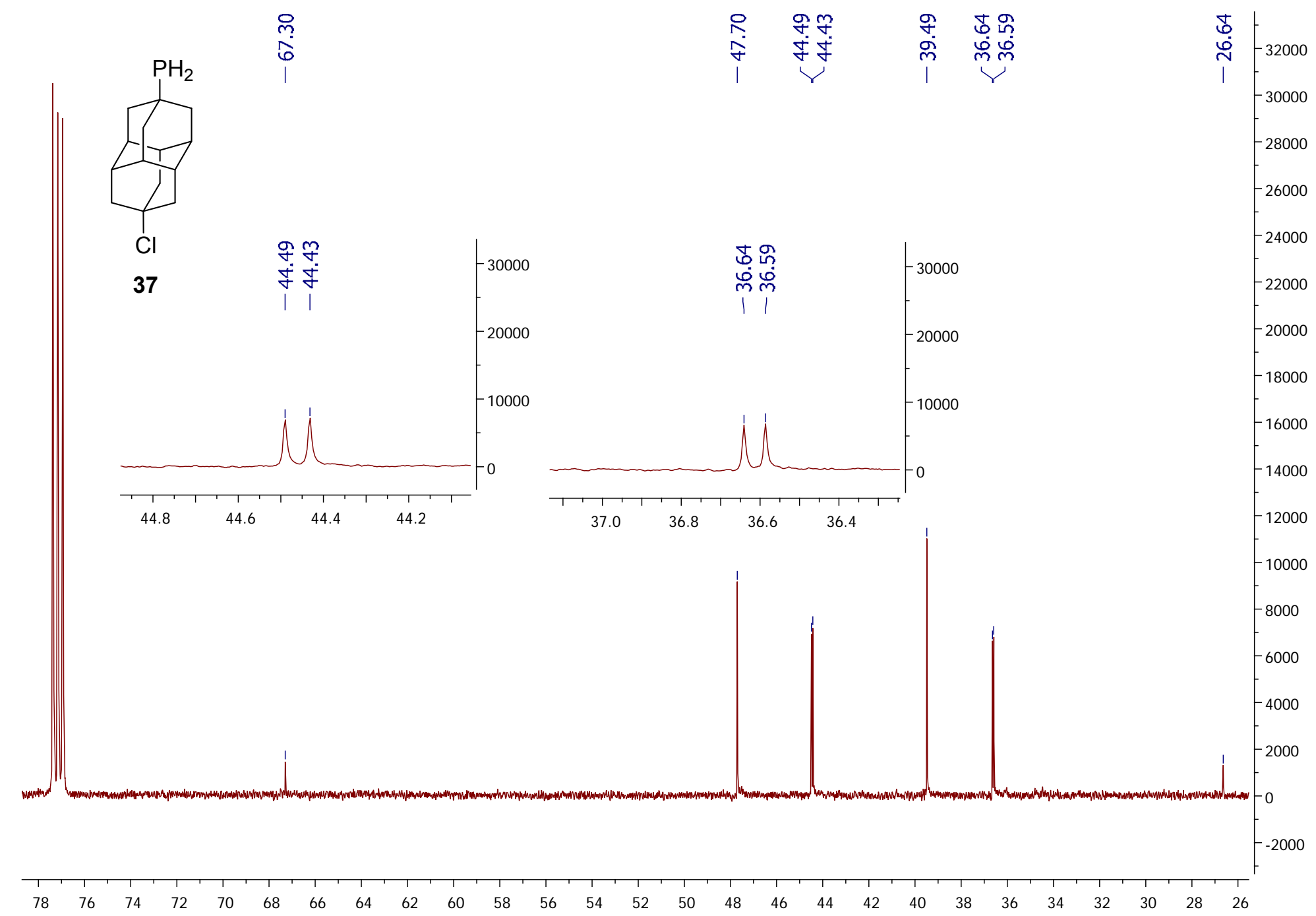


1.18.3. 9-Cl-dia-4- $\mathrm{PH}_{2}^{31} \mathrm{P}$

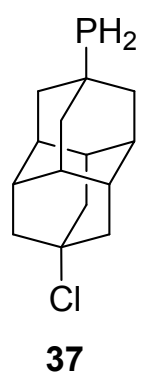

$$
\text { 국오 }
$$

ڤ્山

i

19000

18000

17000

16000

37
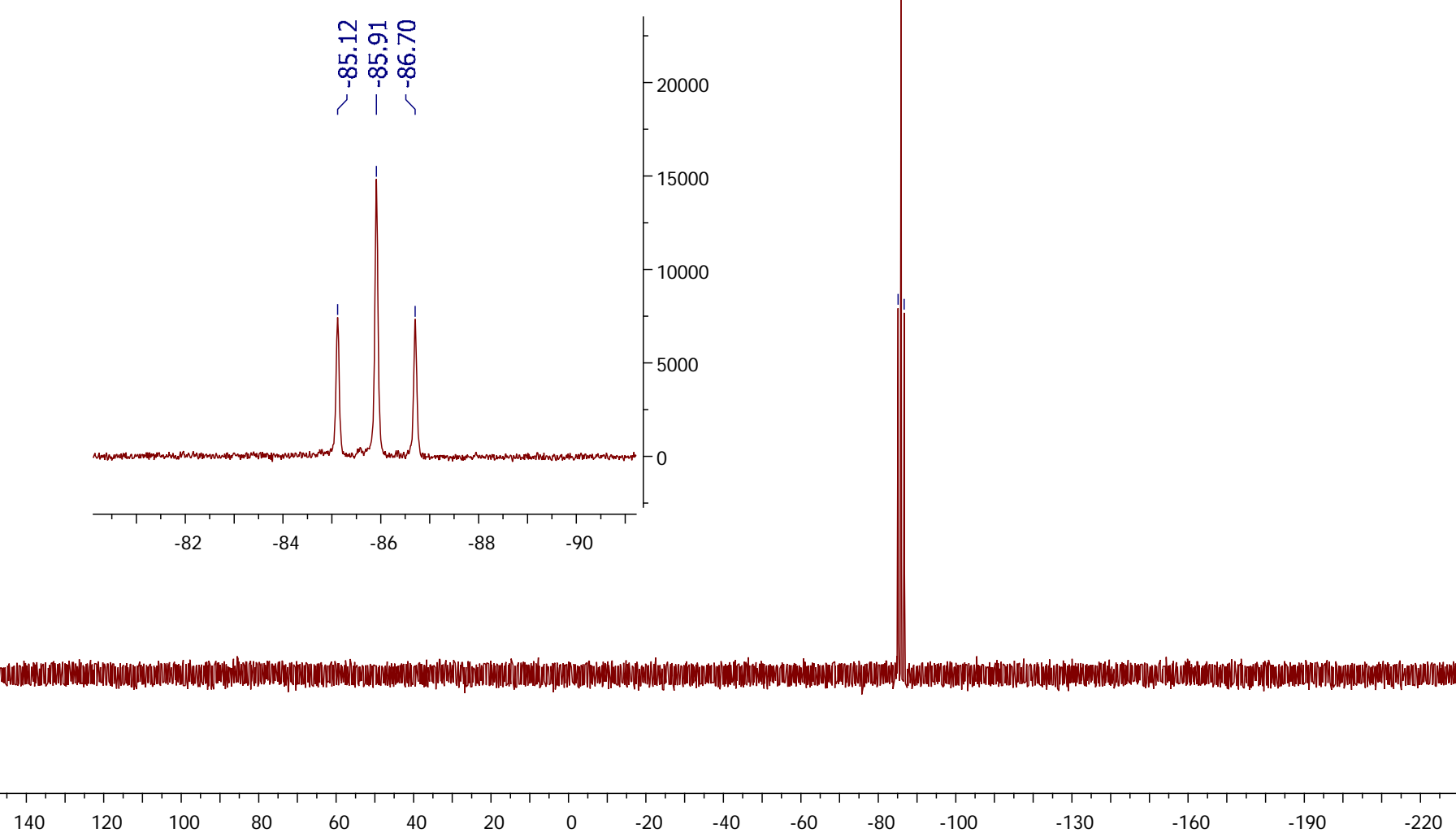
1.18.4. 9-Cl-dia-4- $\mathrm{PH}_{2}{ }^{31} \mathrm{P}\left\{{ }^{1} \mathrm{H}\right\}$

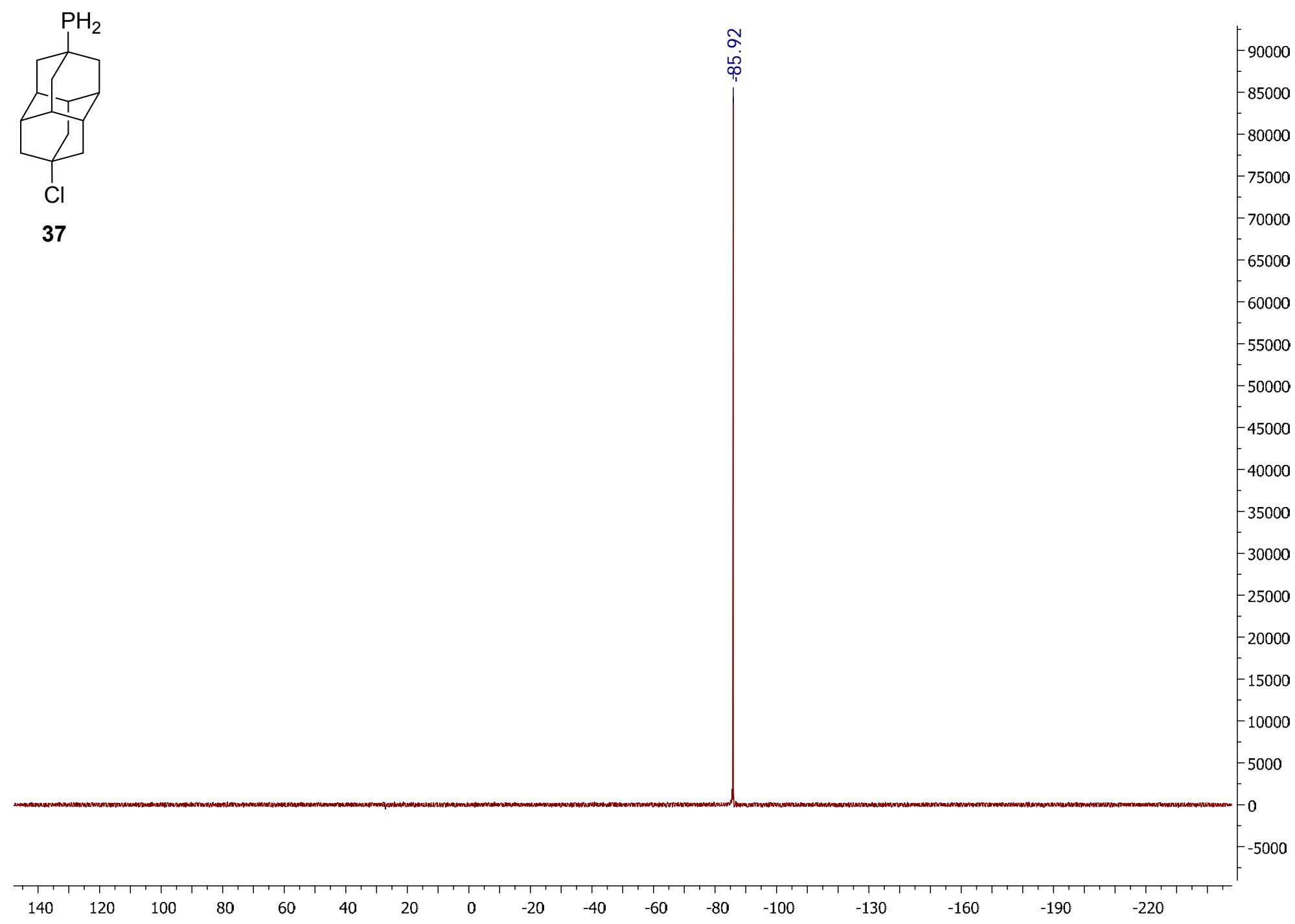


1.19. 4-Diamantylphenylphosphine (38)

1.19.1. 4-Dia-Ph-PH ${ }^{1} \mathrm{H}$

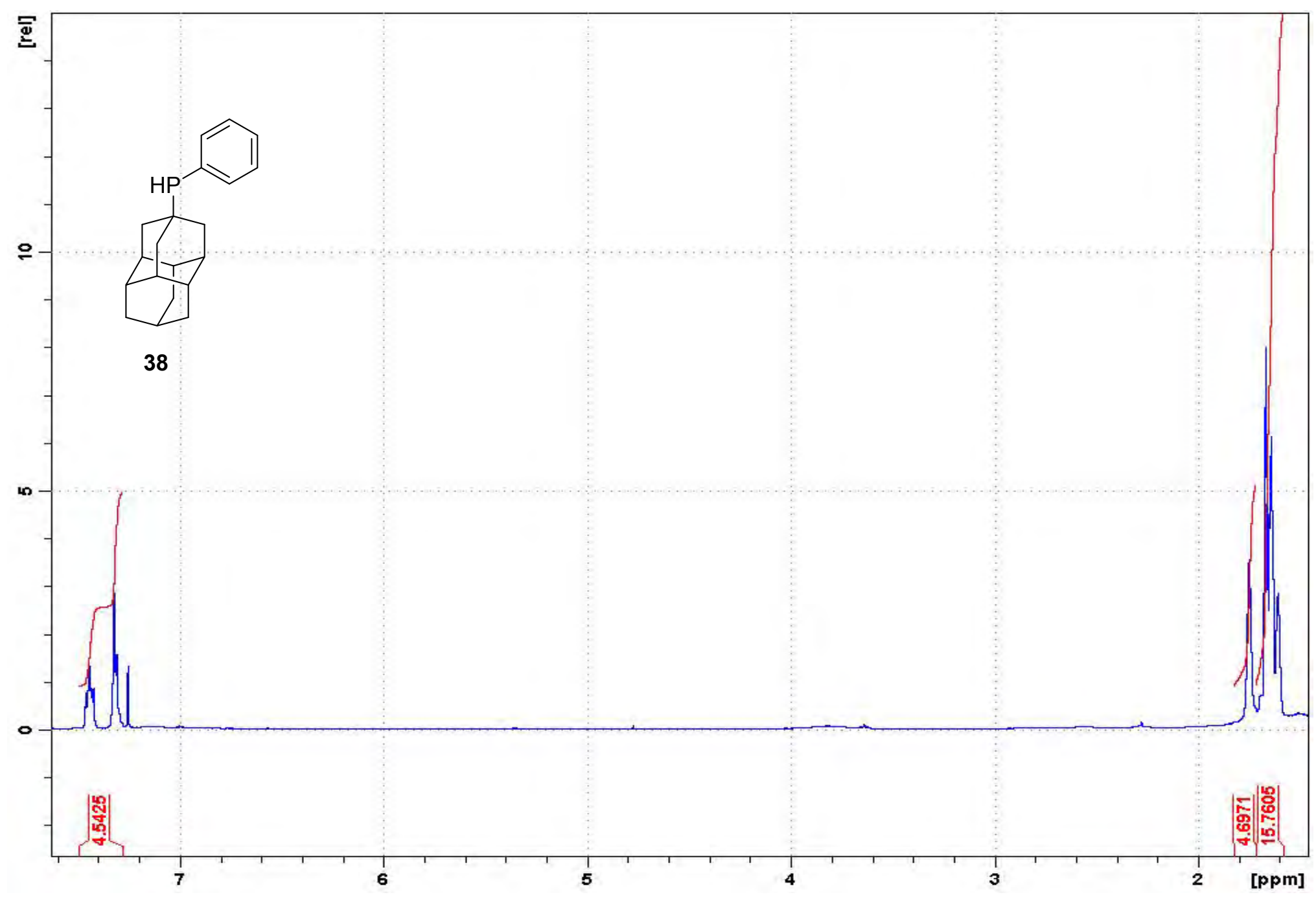


1.19.2. 4-Dia-Ph-PH ${ }^{13} \mathrm{C}$

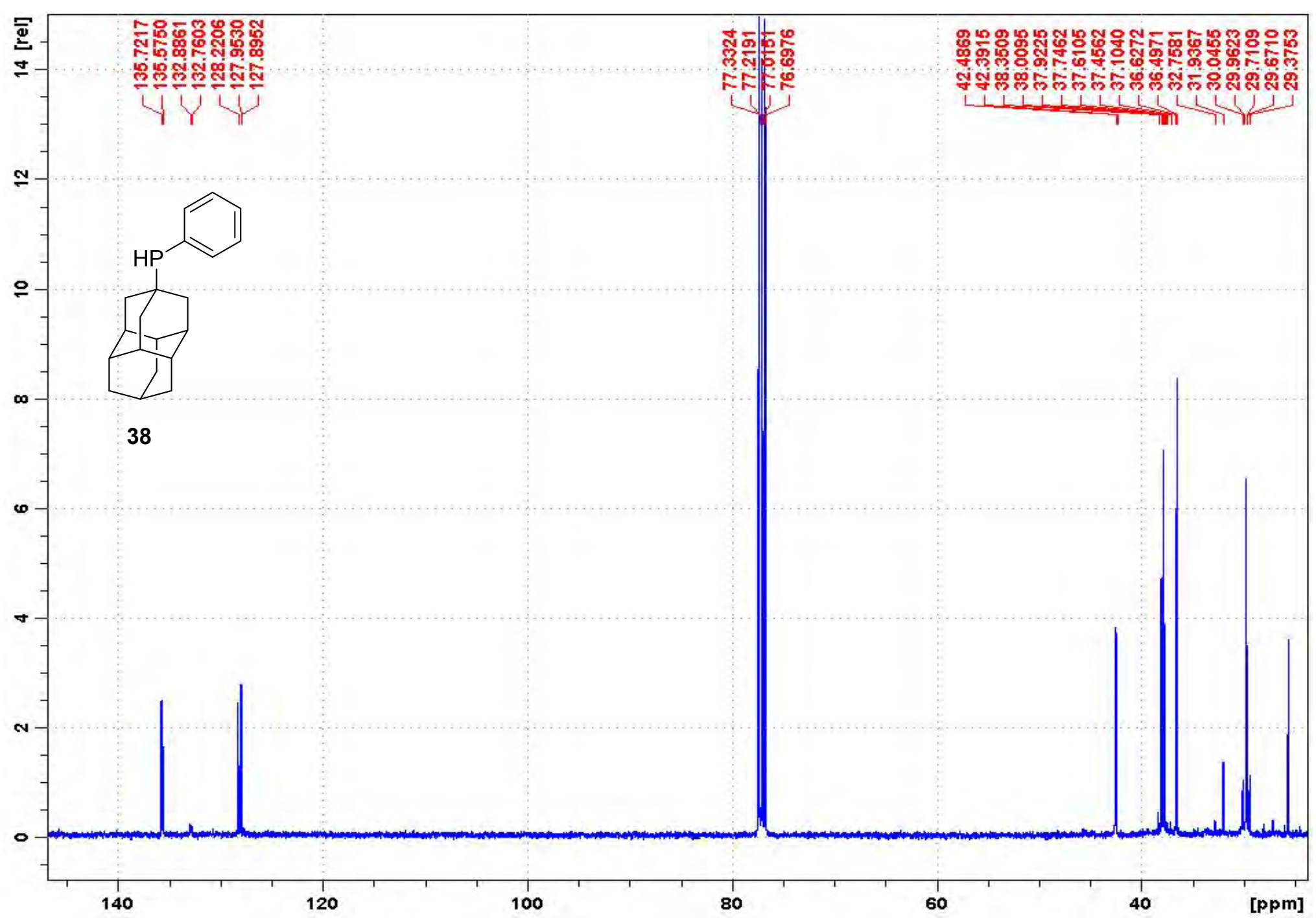


1.19.3. 4-Dia-Ph-PH ${ }^{31} \mathrm{P}$ at $243 \mathrm{~K}$

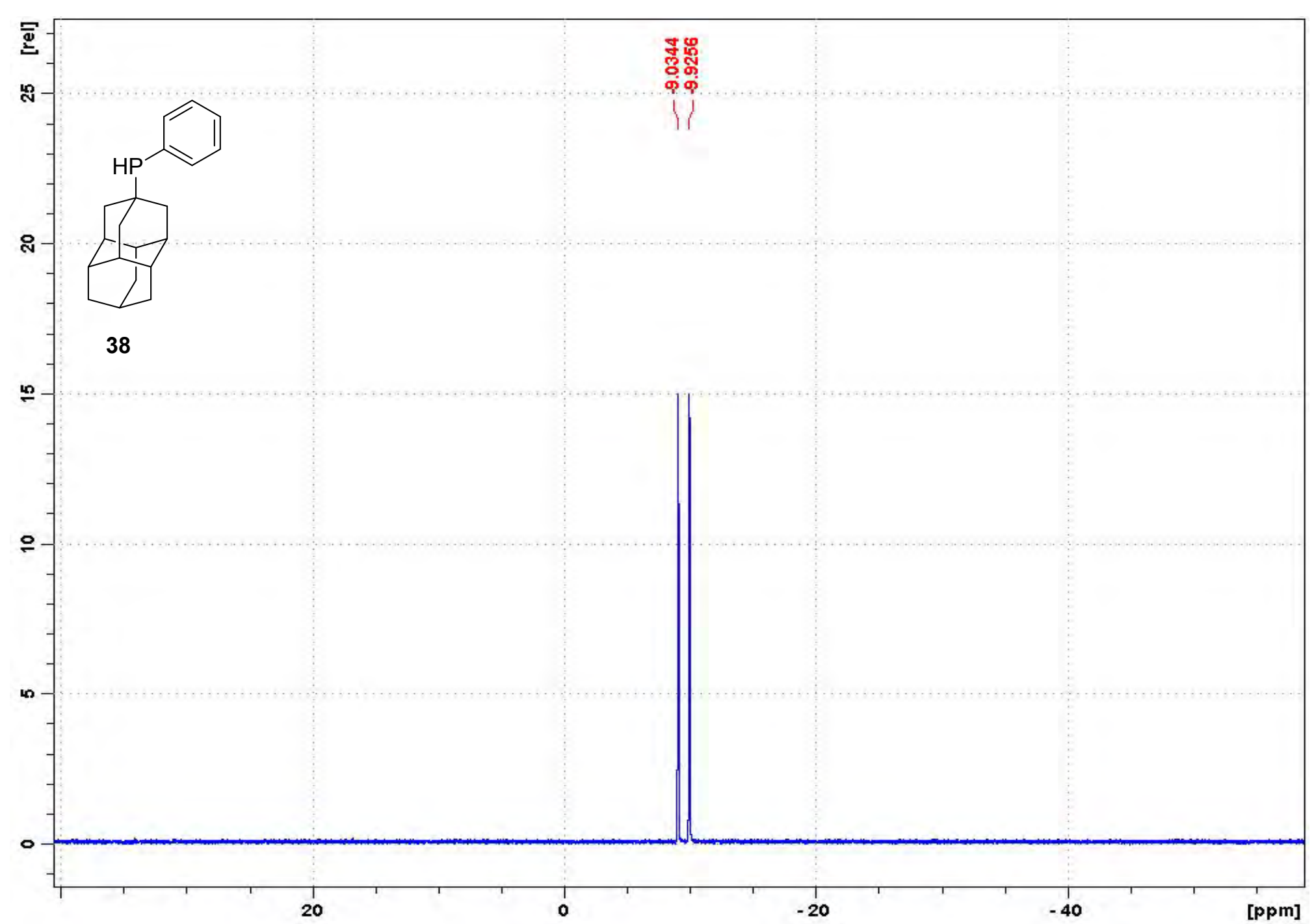


1.19.4. 4-Dia-Ph-PH ${ }^{31} \mathrm{P}$ at $291 \mathrm{~K}$

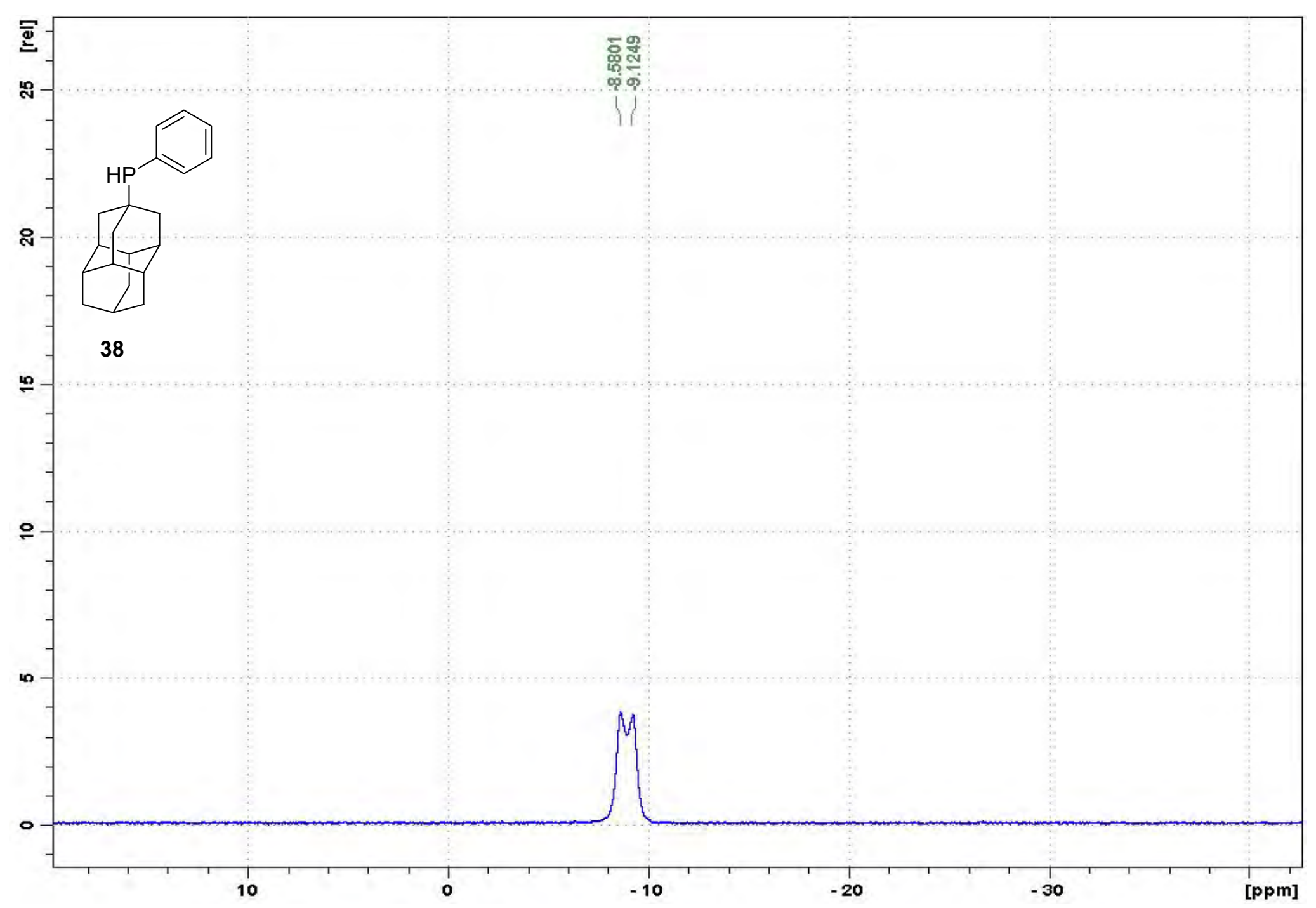


1.19.5. 4-Dia-Ph-PH ${ }^{31} \mathrm{P}$ at $293 \mathrm{~K}$

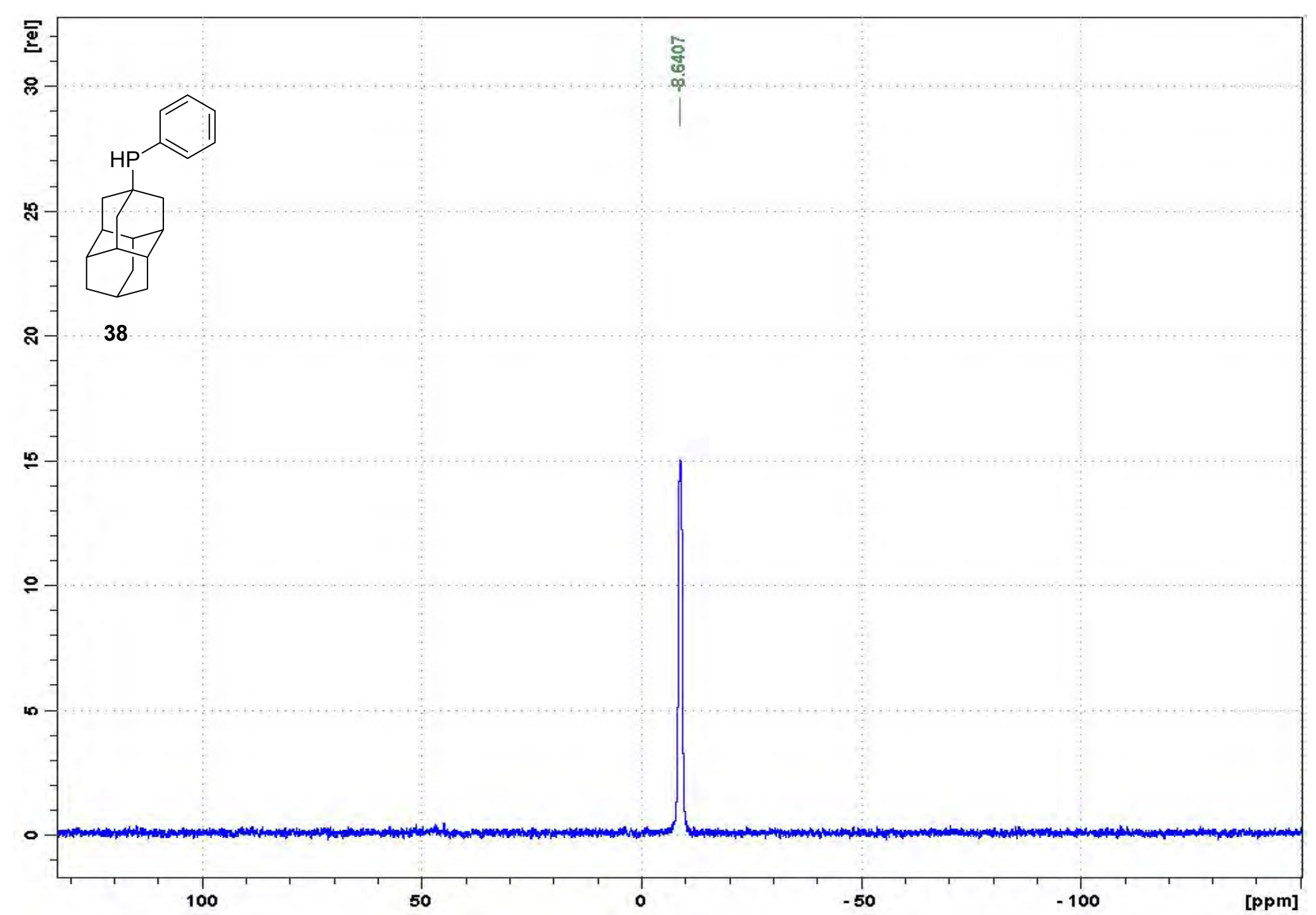


1.19.6. 4-Dia-Ph-PH ${ }^{31} \mathrm{P}$ at $300 \mathrm{~K}$

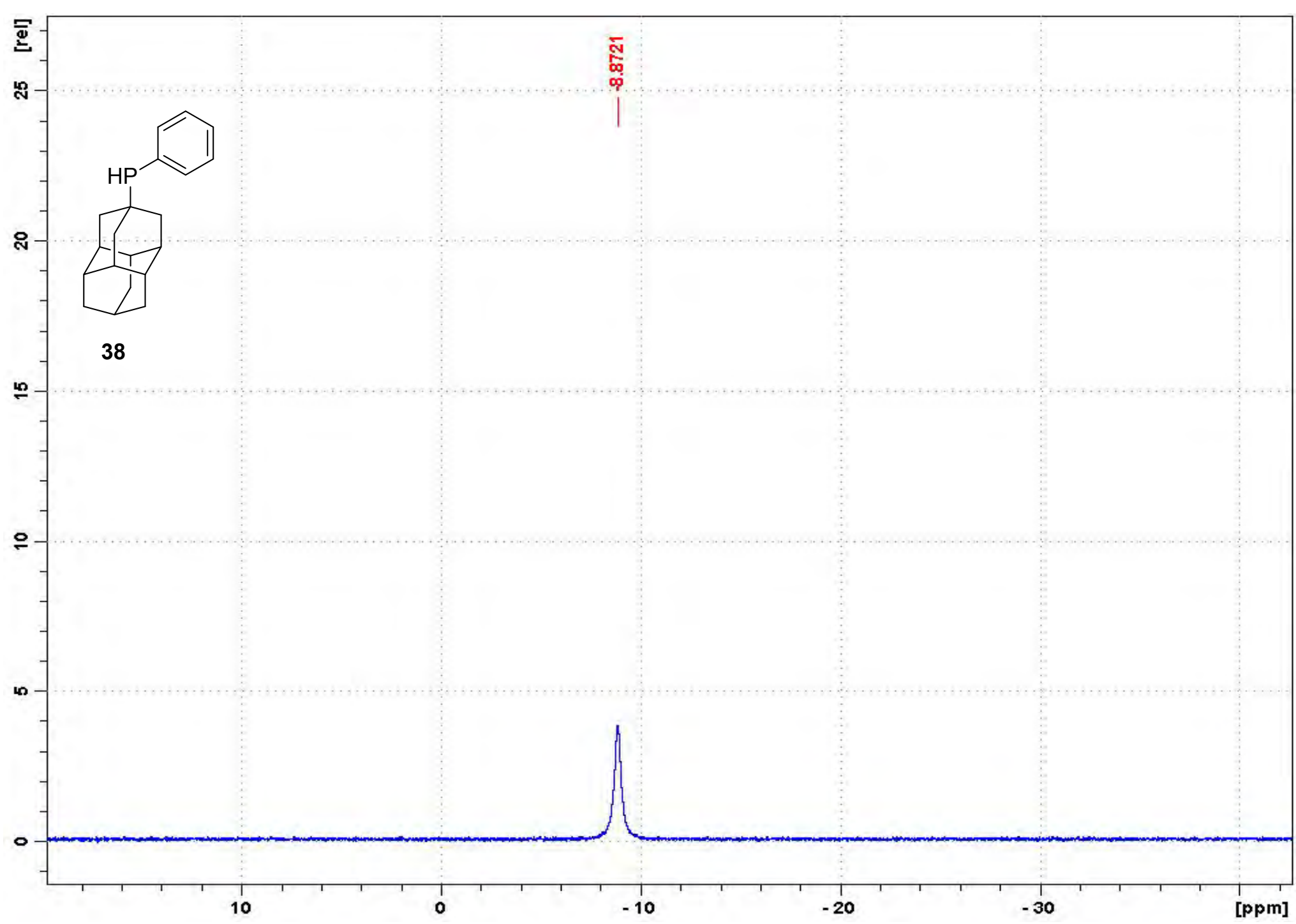


1.20. 1-Diamantylphenylphosphine (39)

1.20.1. 1-Dia-Ph-PH ${ }^{1} \mathrm{H}$ at $253 \mathrm{~K}$

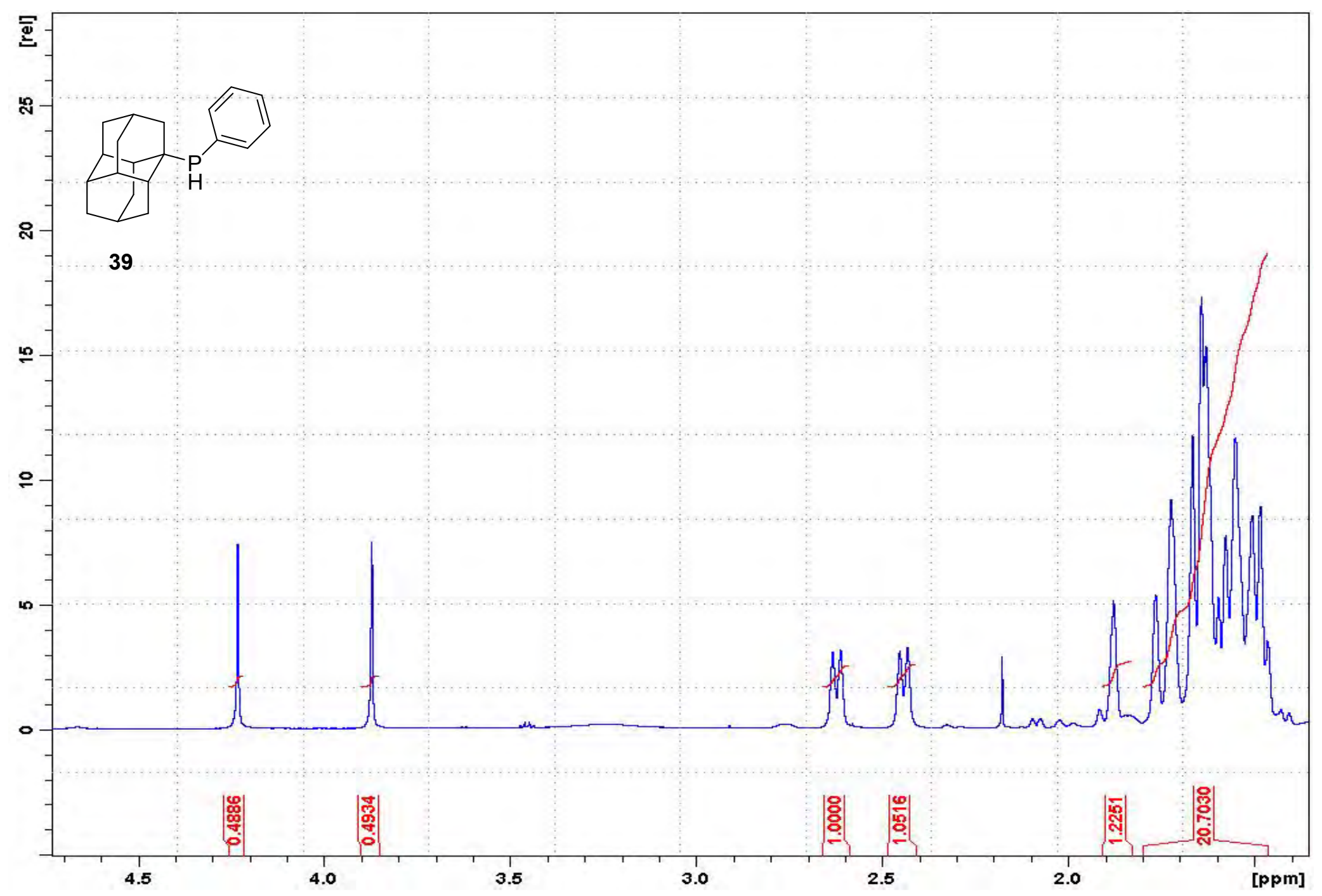


1.20.2. 1-Dia-Ph-PH ${ }^{13} \mathrm{C}$

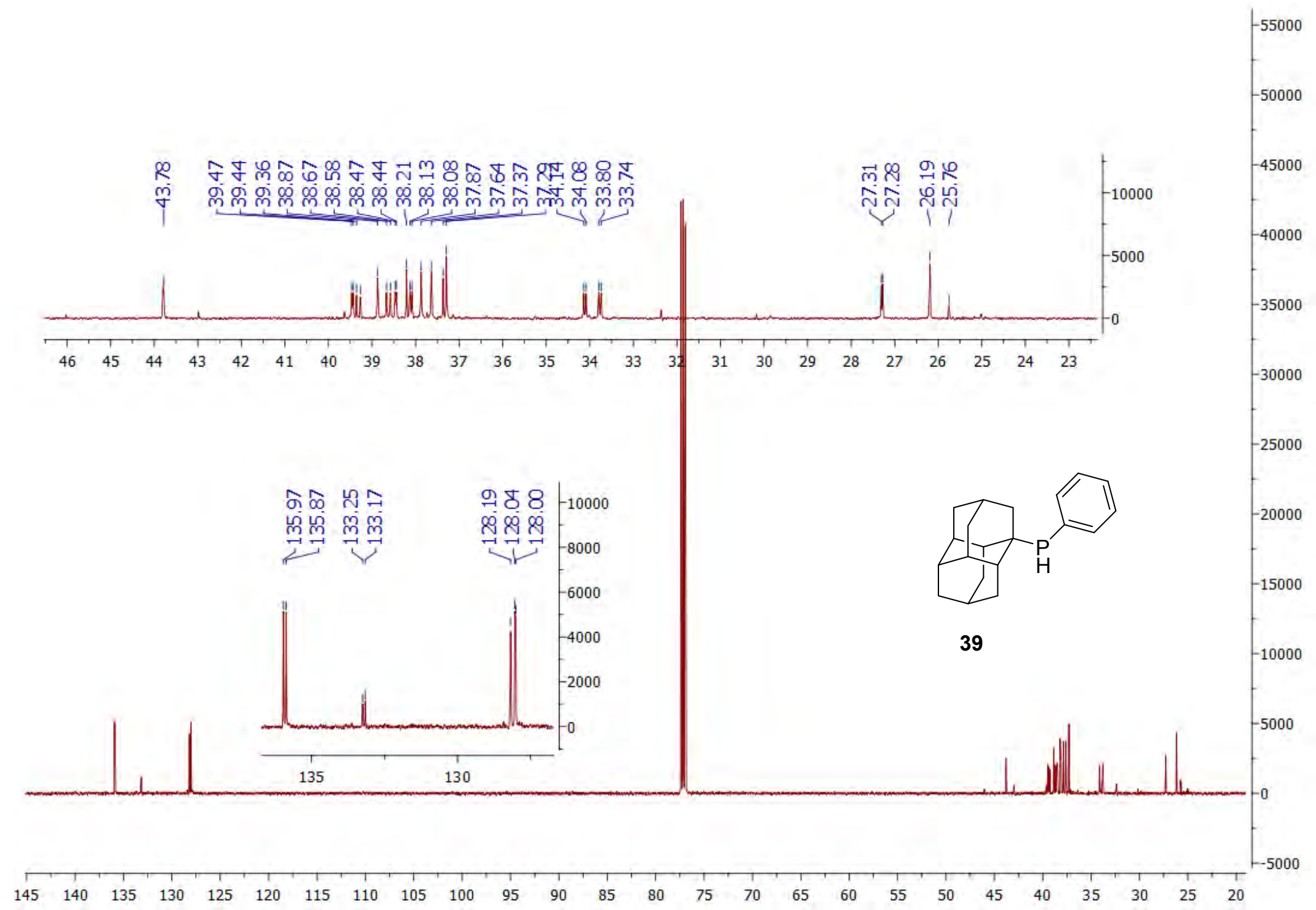


1.20.3. 1-Dia-Ph-PH ${ }^{31} \mathrm{P}$ at $253 \mathrm{~K}$

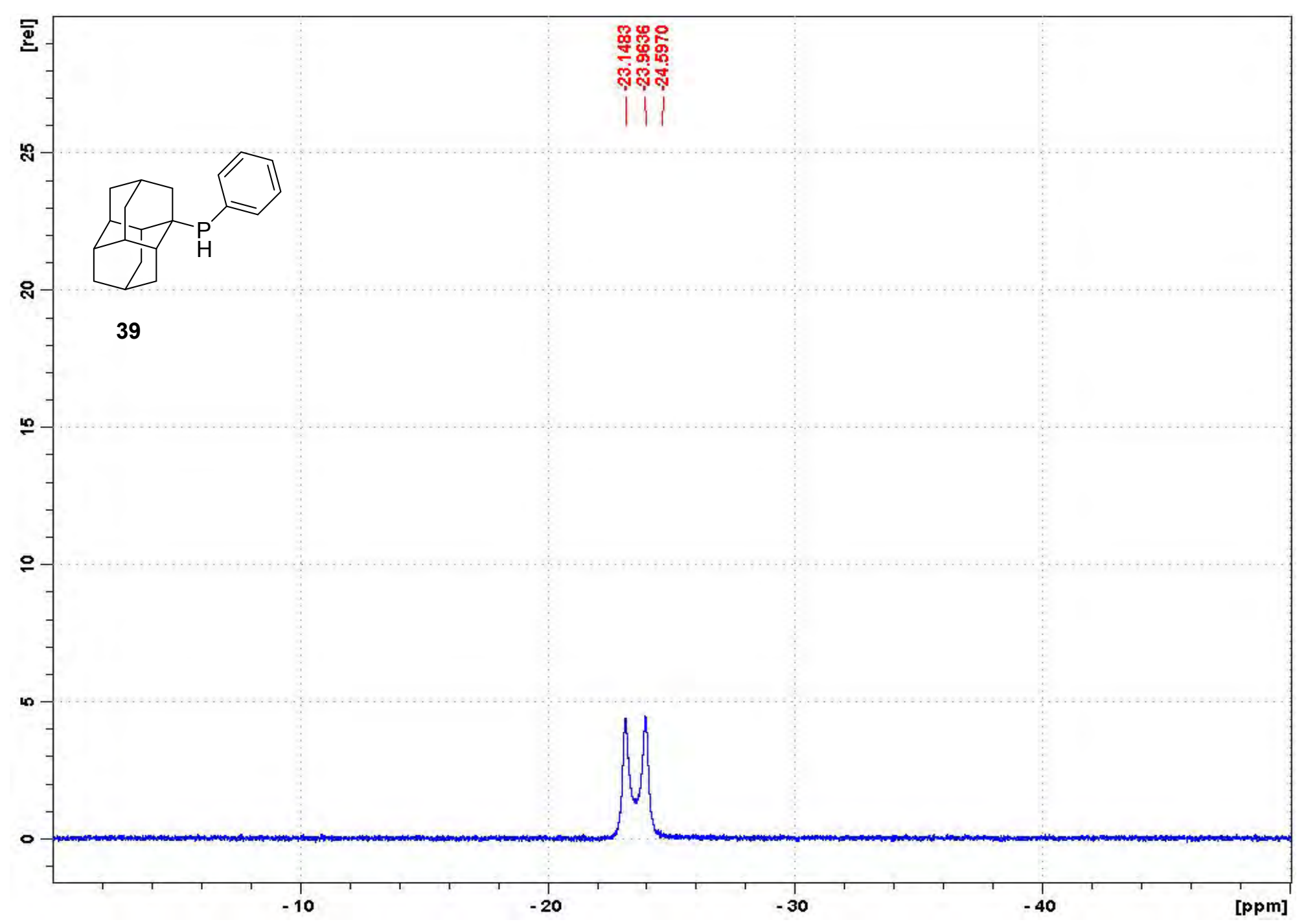


1.21. Tribenzyl(9-hydroxydiamantan-4-yl)phosphonium bromide (40)

1.21.1. triBn-(9-OH-dia-4) $\mathrm{P}^{+} \mathrm{Br}^{-1} \mathrm{H}$

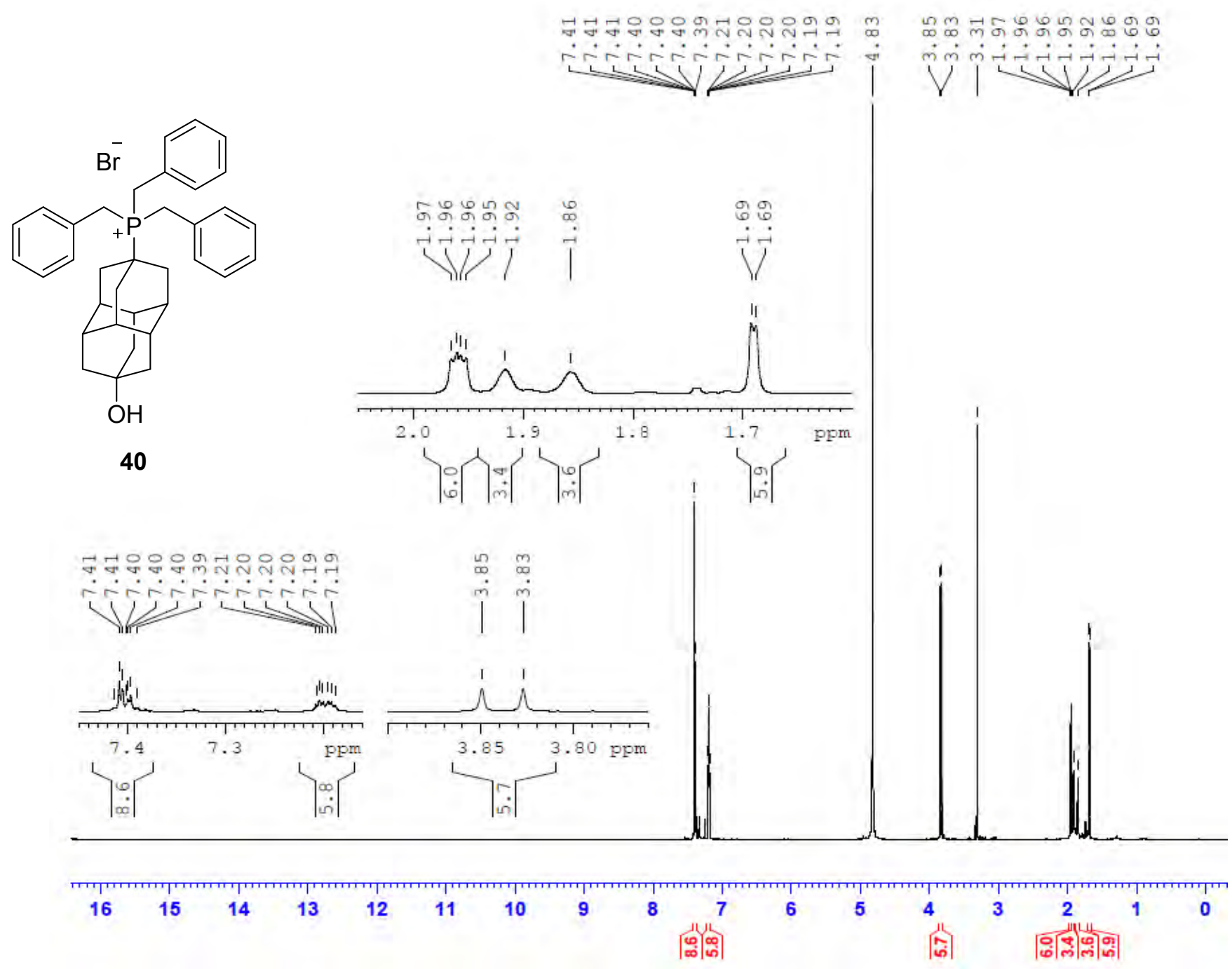


1.21.2. triBn-(9-OH-dia-4) $\mathrm{P}^{+} \mathrm{Br}^{-13} \mathrm{C}$
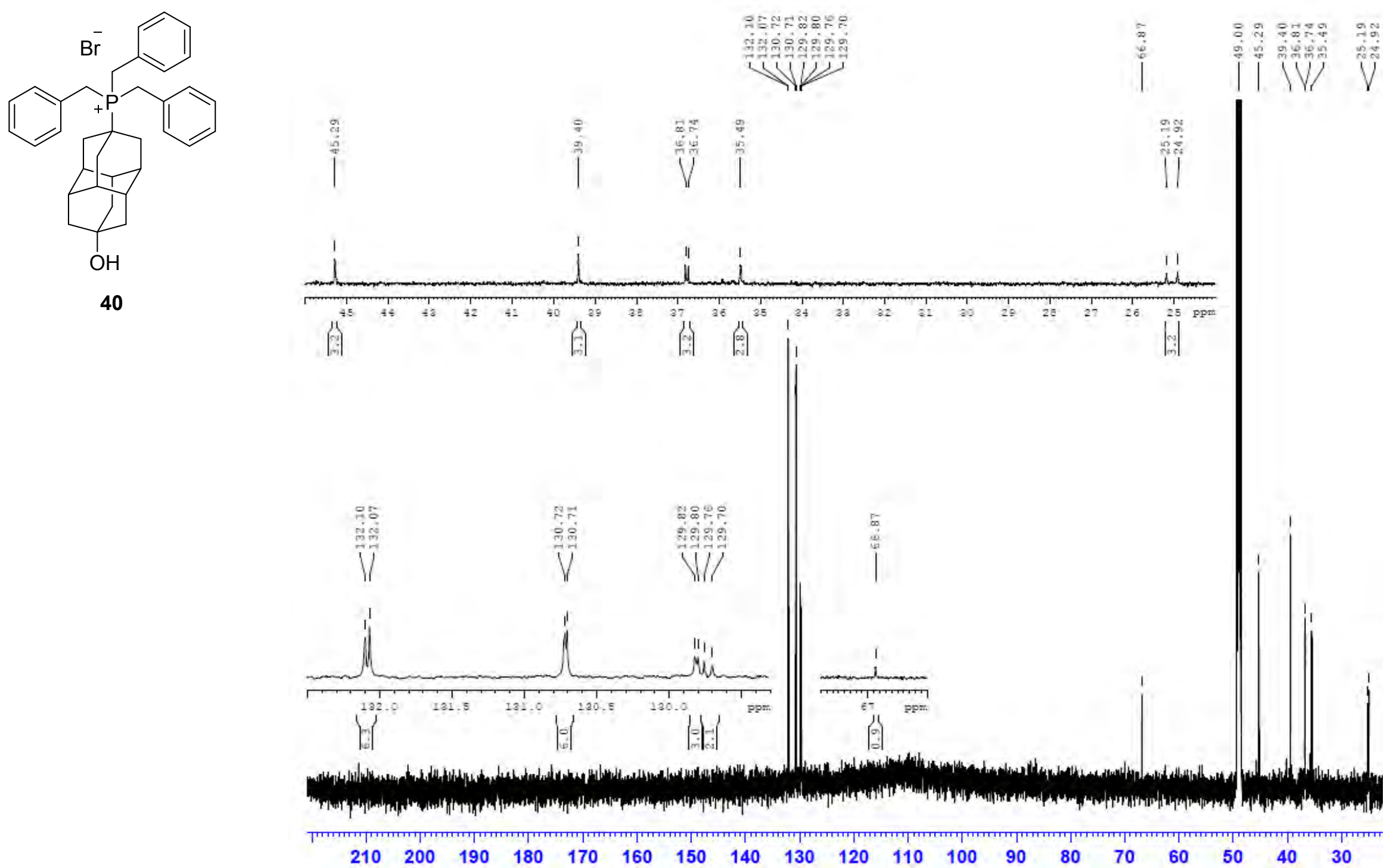
1.21.3. triBn-(9-OH-dia-4) $\mathrm{P}^{+} \mathrm{Br}^{-31} \mathrm{P}$

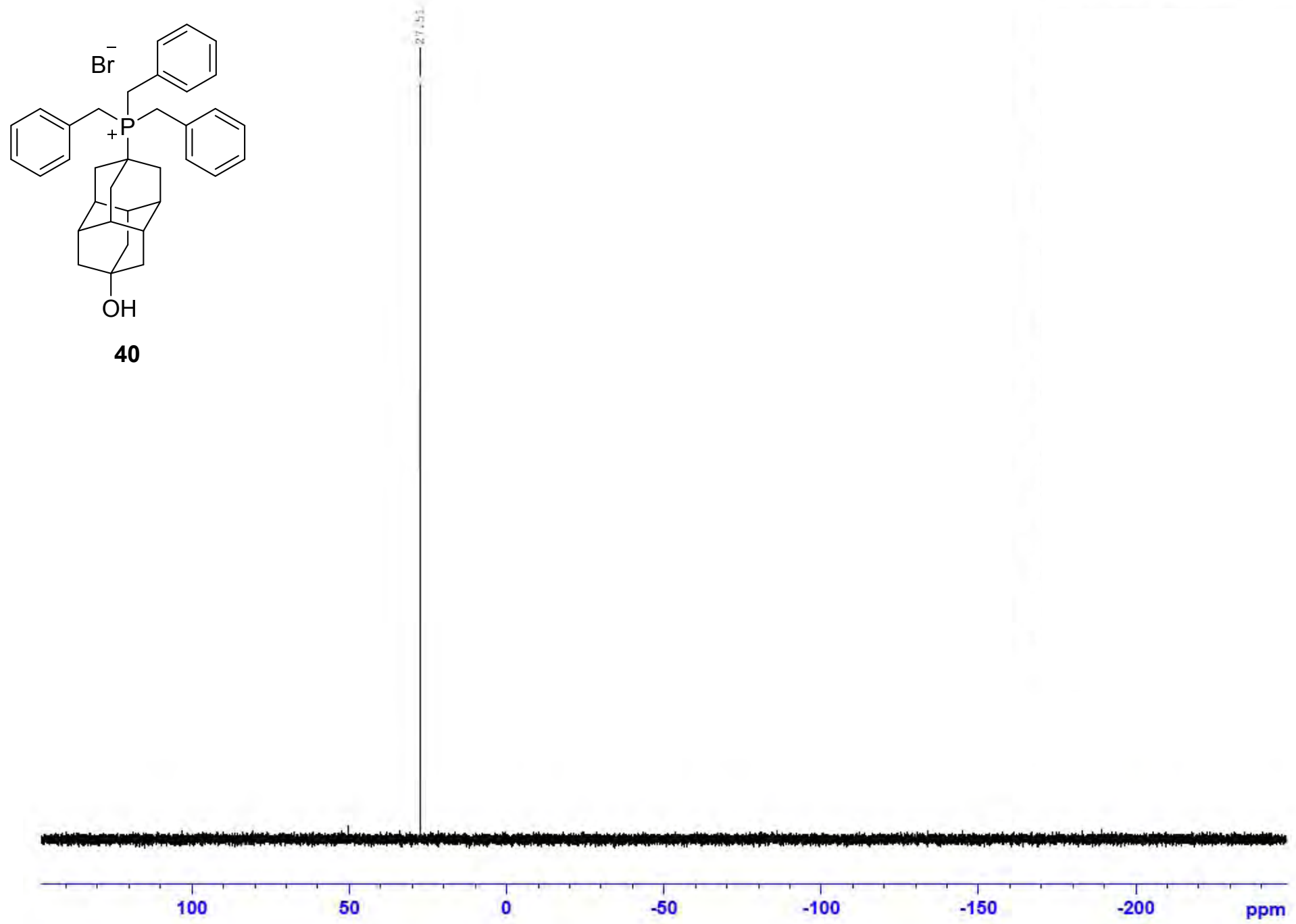


1.22. 1-Adamantyldiphenylphosphine oxide (41)

1.22.1. 1-Ad-P(=O) $\mathrm{Ph}_{2}{ }^{1} \mathrm{H}$

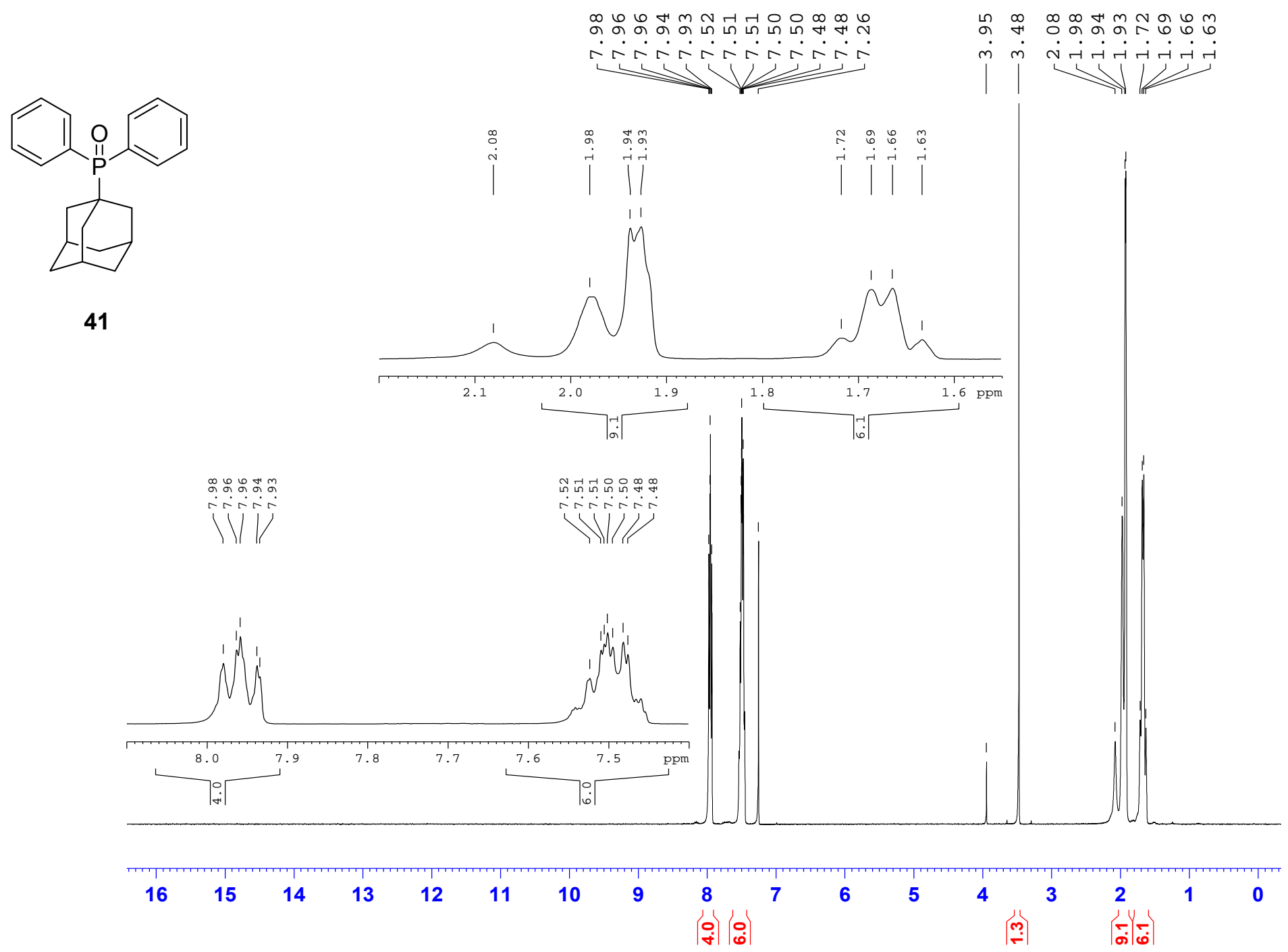


1.22.2. $1-\mathrm{Ad}-\mathrm{P}(=0) \mathrm{Ph}_{2}{ }^{13} \mathrm{C}$

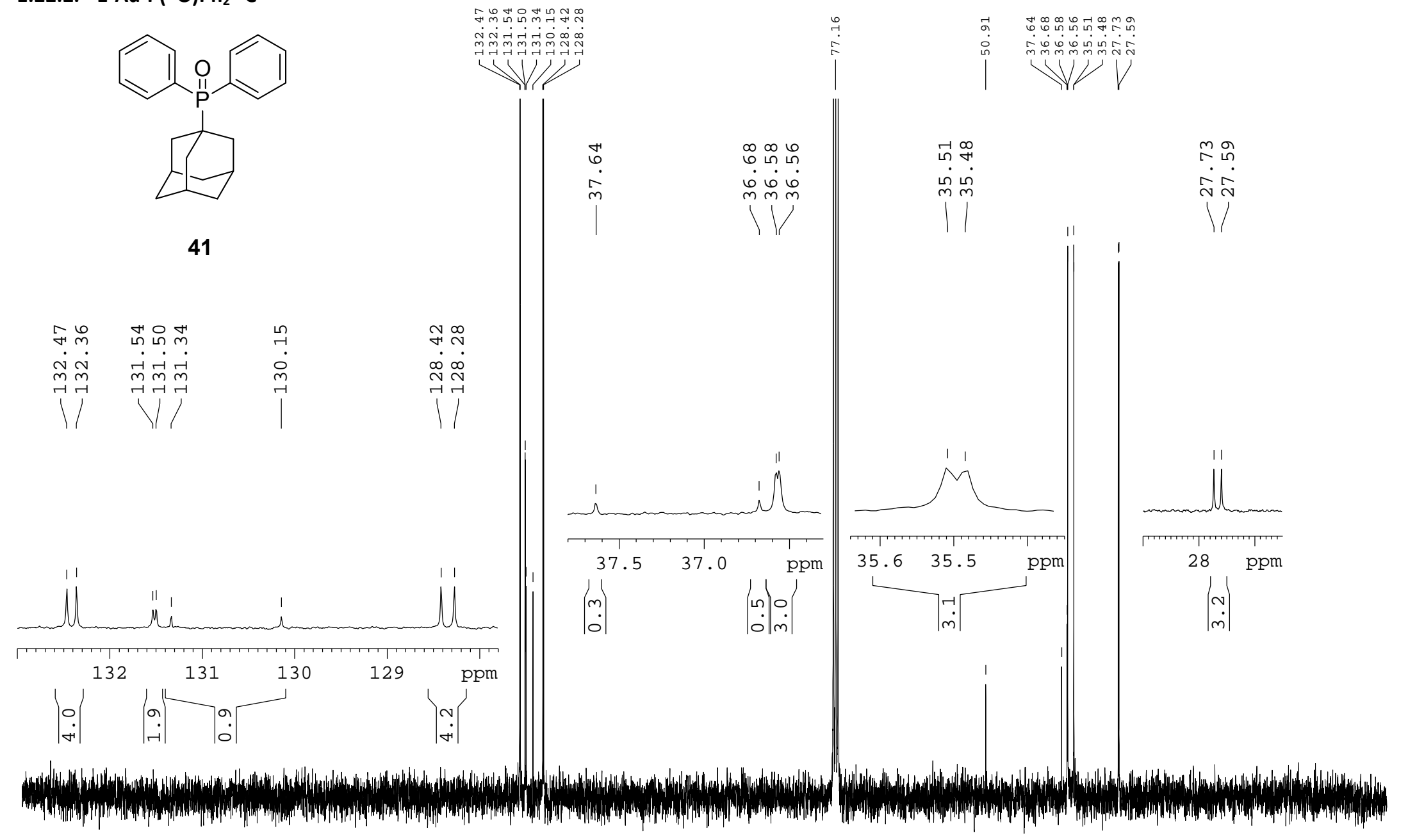

$\begin{array}{lllllllllllllllllllllll}210 & 200 & 190 & 180 & 170 & 160 & 150 & 140 & 130 & 120 & 110 & 100 & 90 & 80 & 70 & 60 & 50 & 40 & 30 & 20 & 10 & 0 & \text { ppm }\end{array}$ 
1.22.3. $1-\mathrm{Ad}-\mathrm{P}(=0) \mathrm{Ph}_{2}{ }^{31} \mathrm{P}$

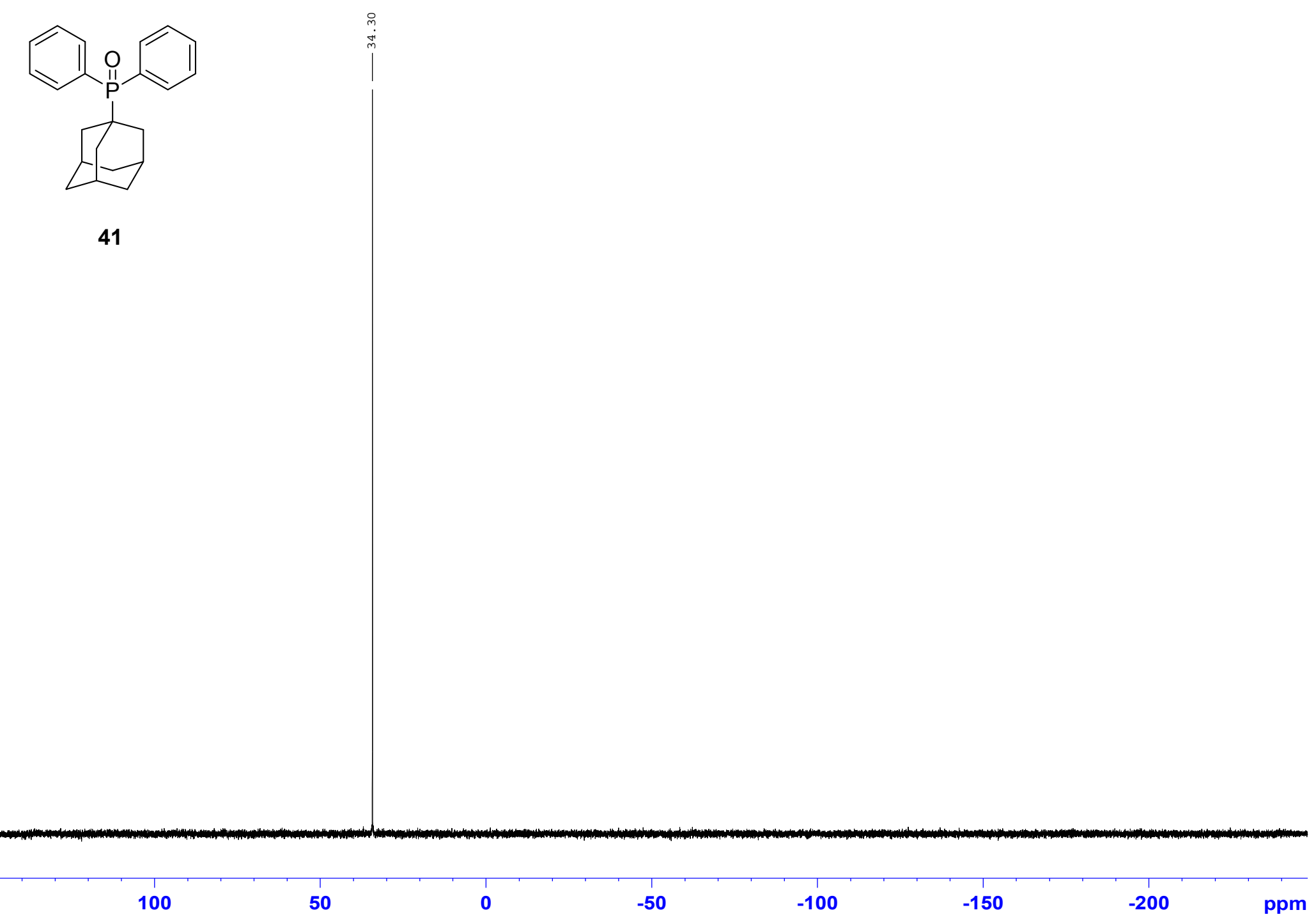


1.23. (3-Hydroxyadamant-1-yl)diphenylphosphine oxide (42)

1.23.1. 3-OH-ad-1-P(=O) $\mathrm{Ph}_{2}{ }^{1} \mathrm{H}$

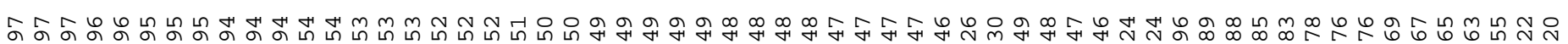

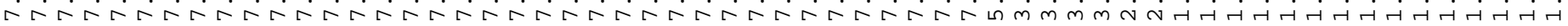

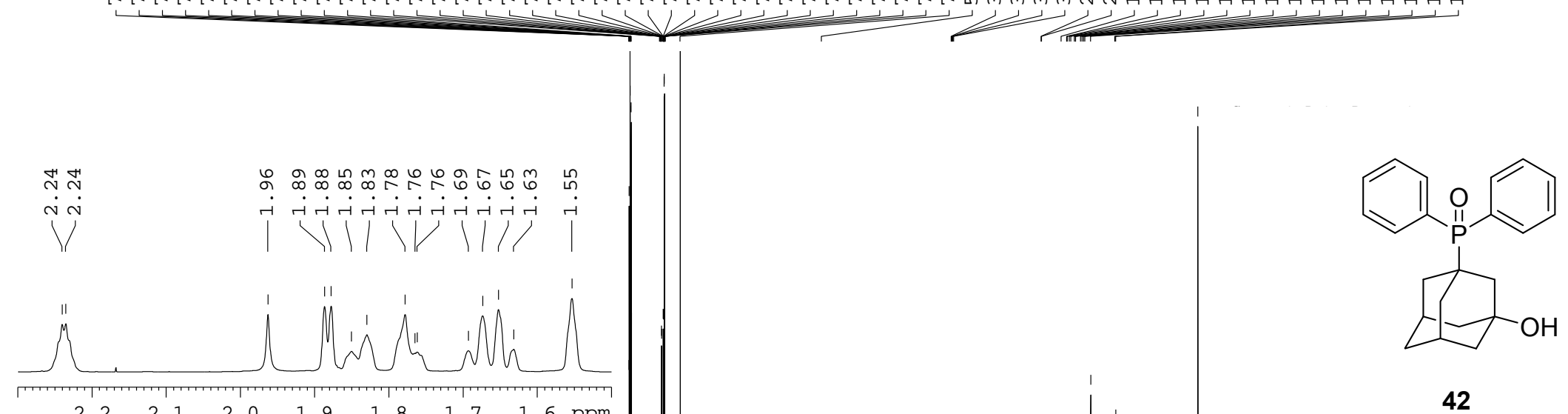

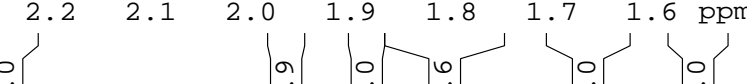

ii

๑)

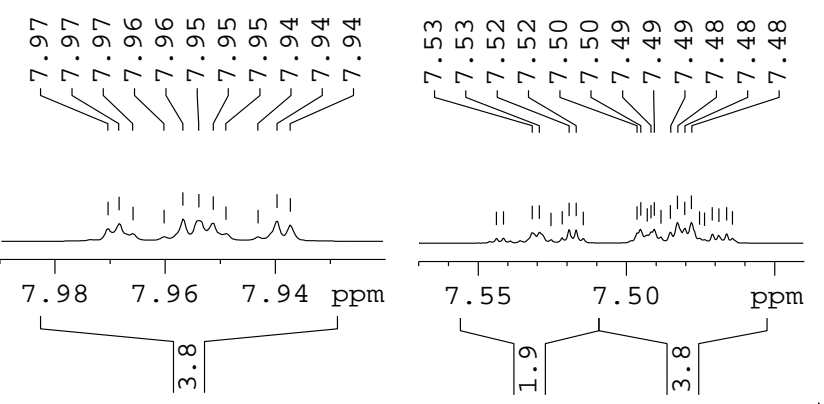

42

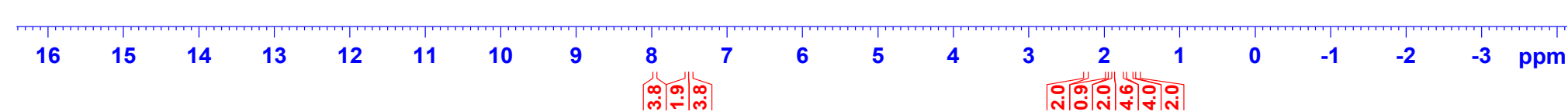


1.23.2. $3-\mathrm{OH}-\mathrm{ad}-1-\mathrm{P}(=0) \mathrm{Ph}_{2}{ }^{13} \mathrm{C}$

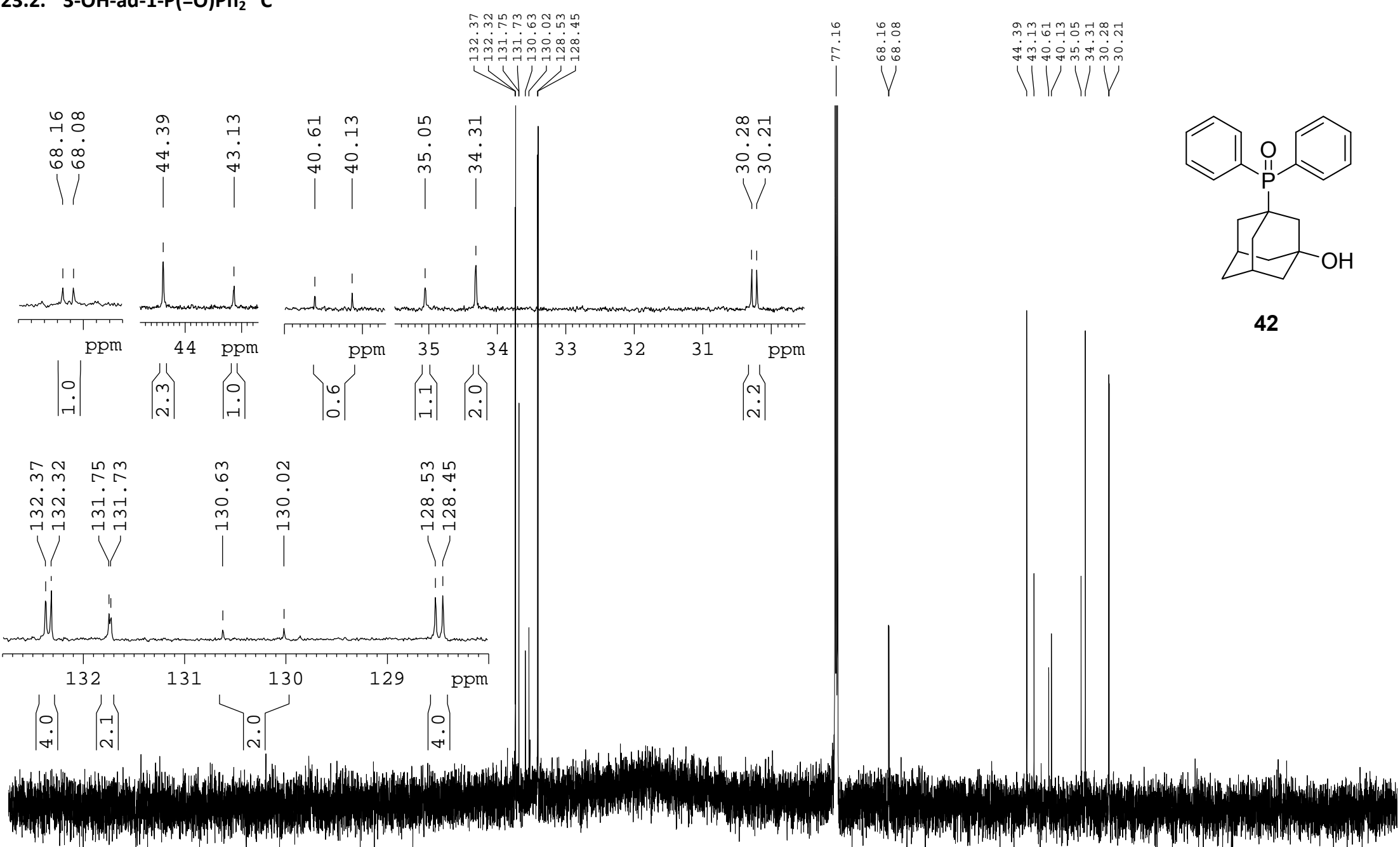

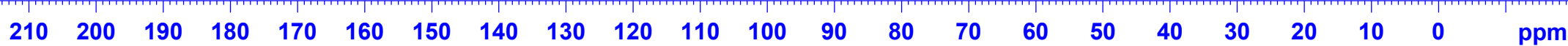


1.23.3. $3-\mathrm{OH}-\mathrm{ad}-1-\mathrm{P}(=0) \mathrm{Ph}_{2}{ }^{31} \mathrm{P}$

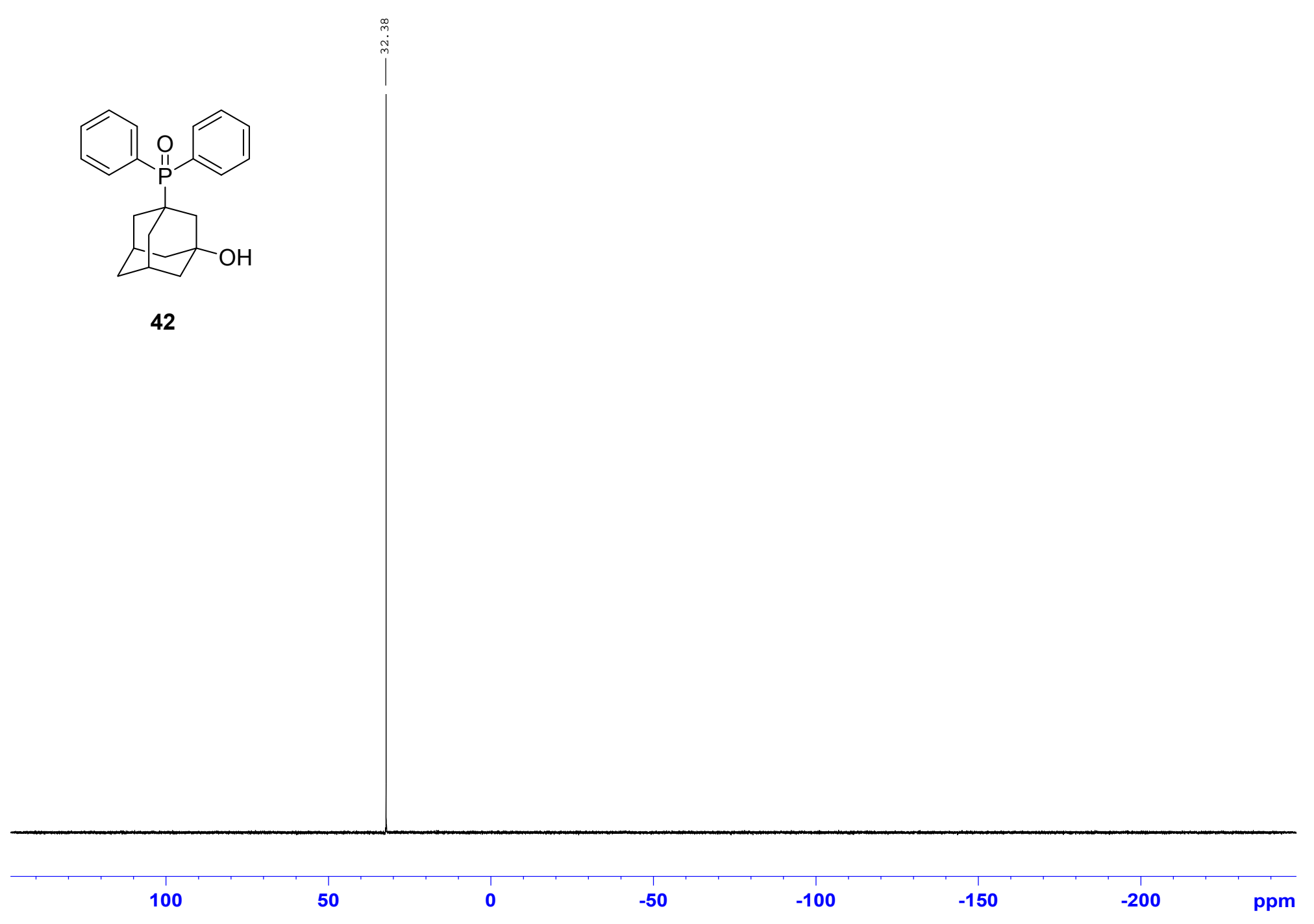


1.24. 4-Diamantyldiphenylphosphine oxide (43)

1.24.1. 4-Dia- $\mathrm{P}(=\mathrm{O}) \mathrm{Ph}_{2}{ }^{1} \mathrm{H}$

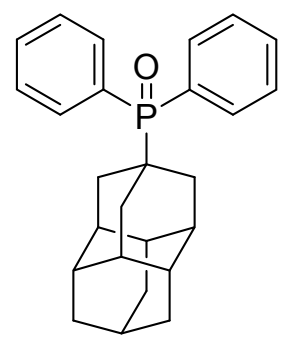

43

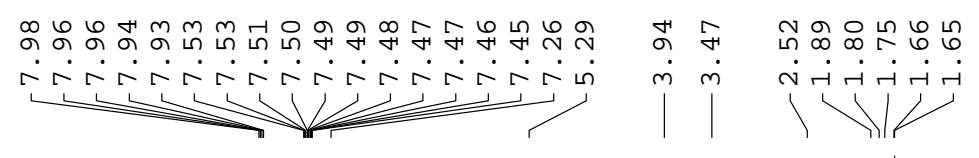

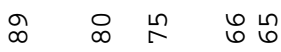
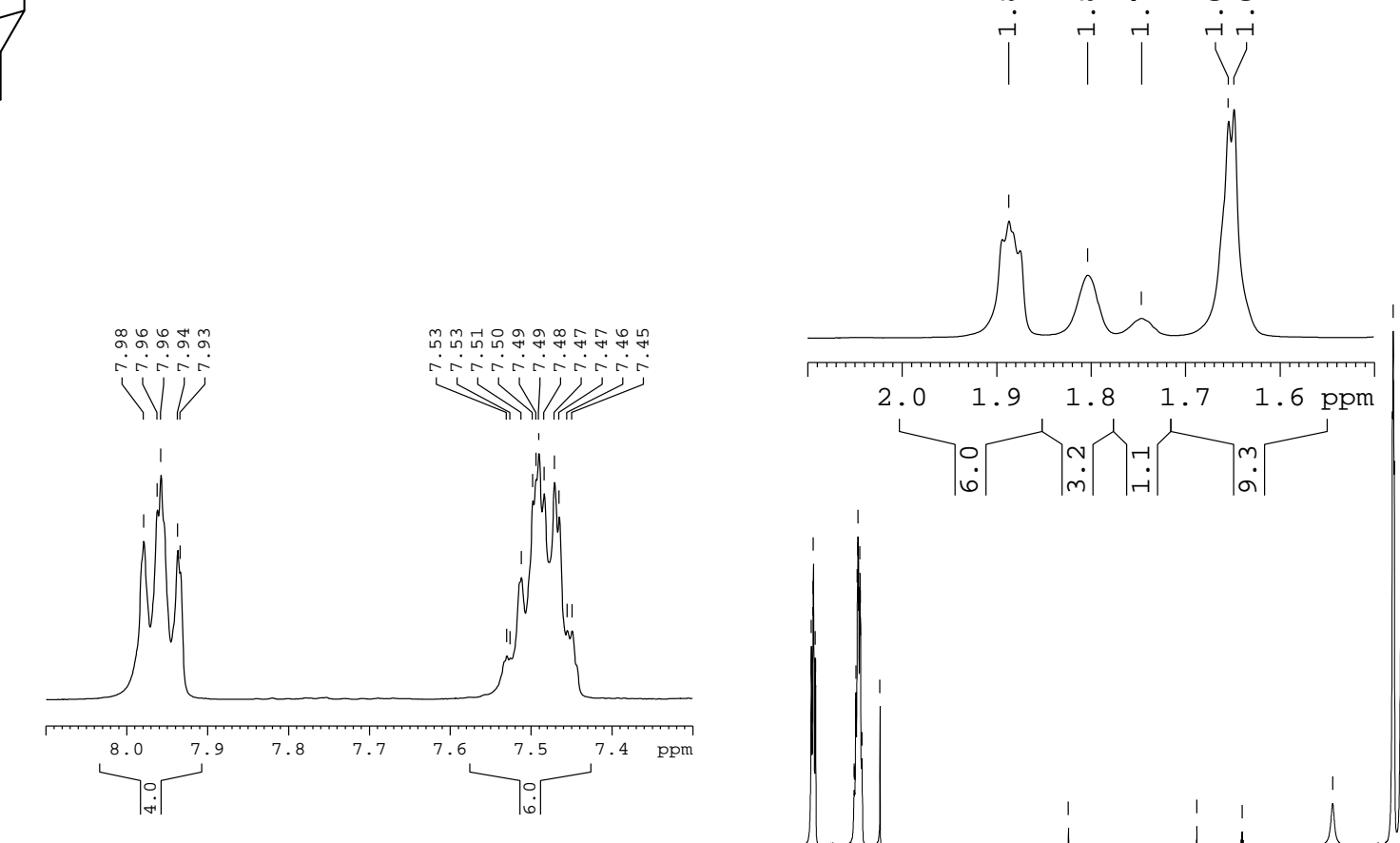

$16 \quad 15$

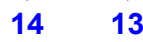

12
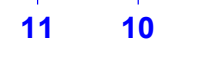

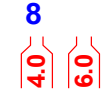

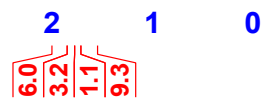


1.24.2. $4-$ Dia- $\mathrm{P}(=0) \mathrm{Ph}_{2}{ }^{13} \mathrm{C}$
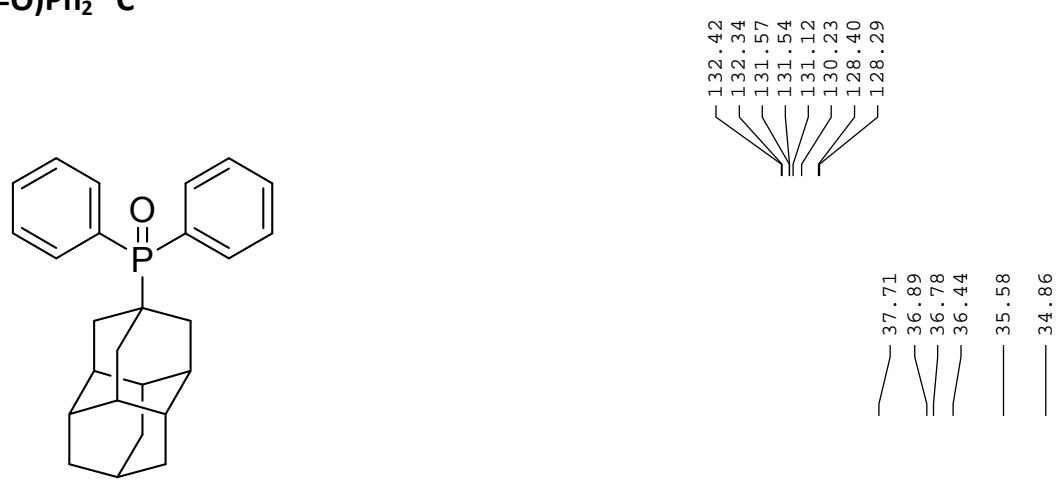

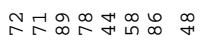

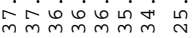

43

||$^{\mid} \mid$

W|/

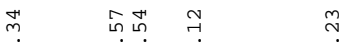

||$_{\mid}^{\dot{m} \vec{ल}}$

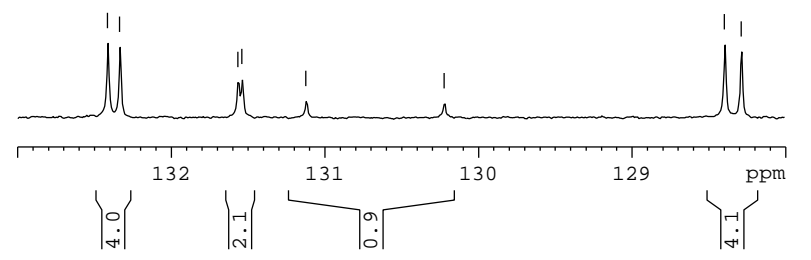

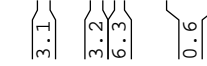

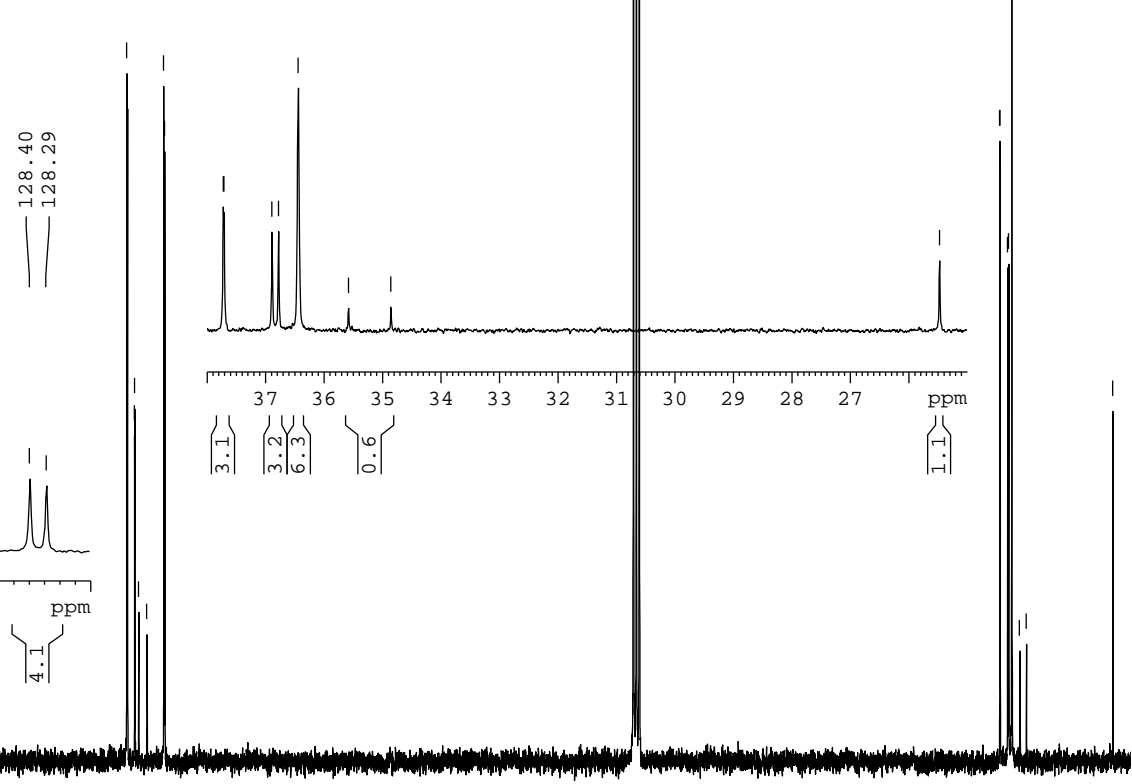

ம்

140130

$\begin{array}{lll}120 & 110 & 100\end{array}$

80

$70 \quad 60$

$\begin{array}{lll}50 & 40 \quad 30\end{array}$ 
1.24.3. $4-$ Dia- $P(=0) \mathrm{Ph}_{2}{ }^{31} \mathrm{P}$

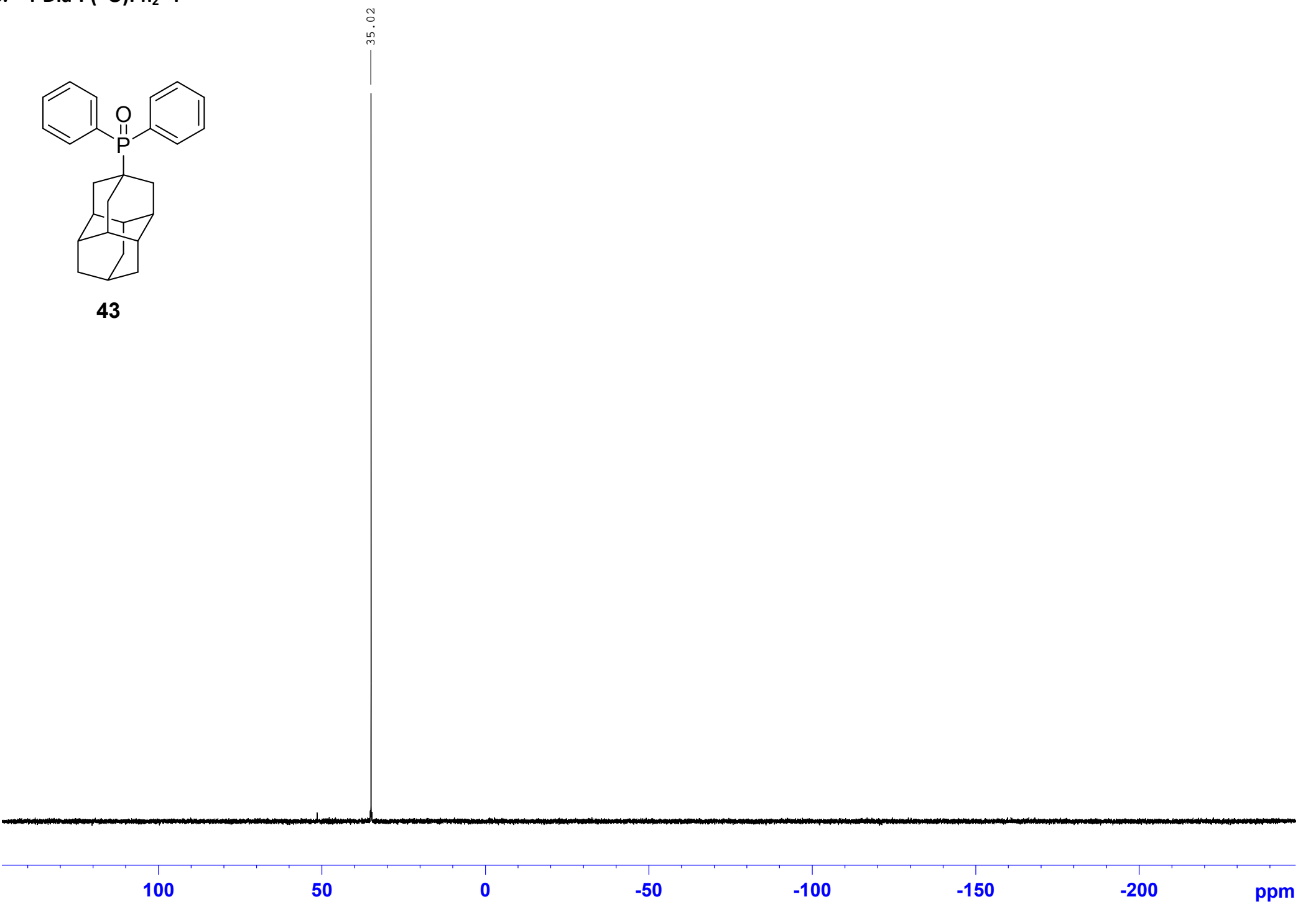


1.25. (9-Hydroxydiamant-4-yl)diphenylphosphineoxide (44)

1.25.1. 9-OH-dia-4- $\mathrm{P}(=\mathrm{O}) \mathrm{Ph}_{2}{ }^{1} \mathrm{H}$

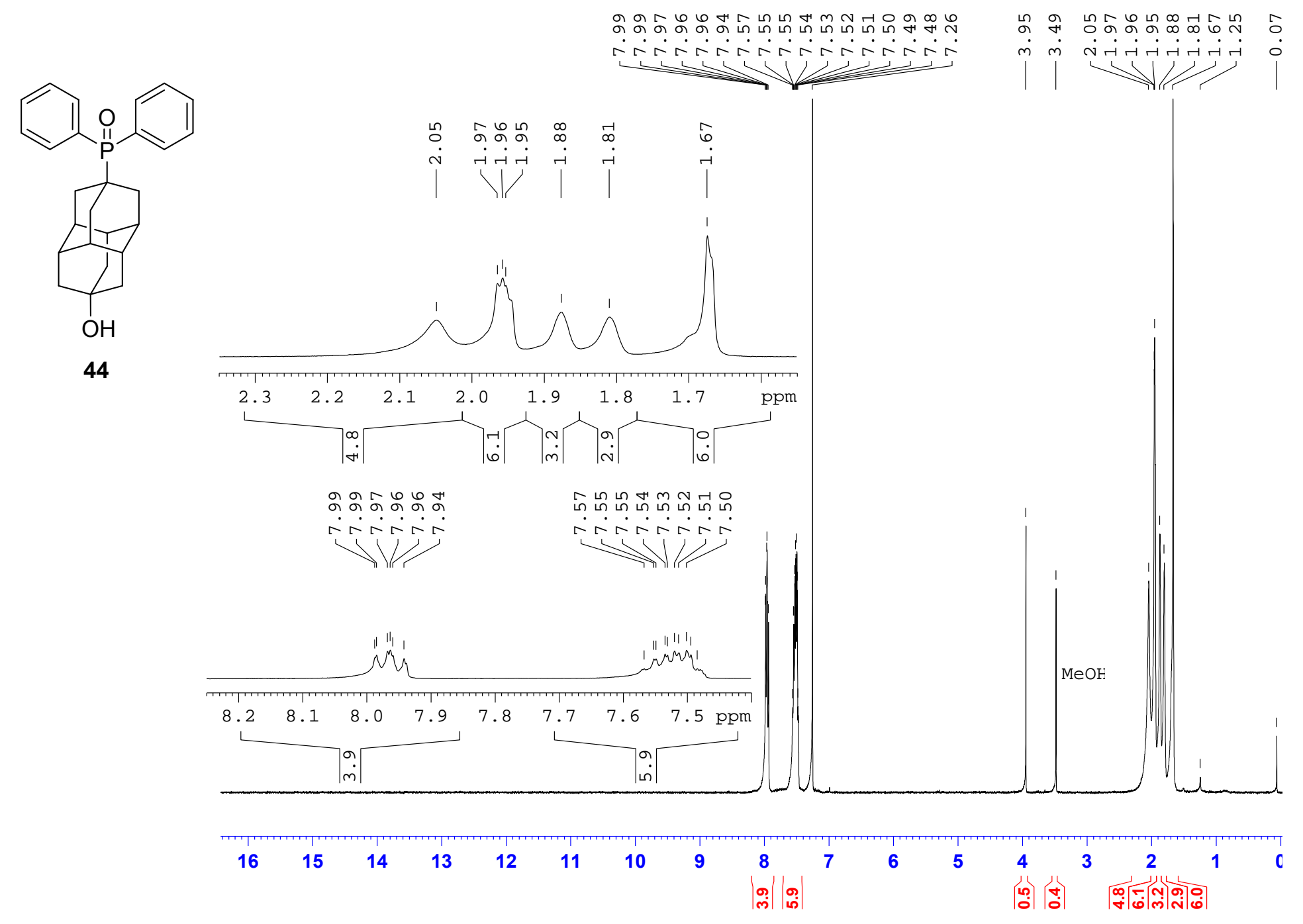


1.25.2. 9-OH-dia-4-P(=O) $\mathrm{Ph}_{2}{ }^{13} \mathrm{C}$

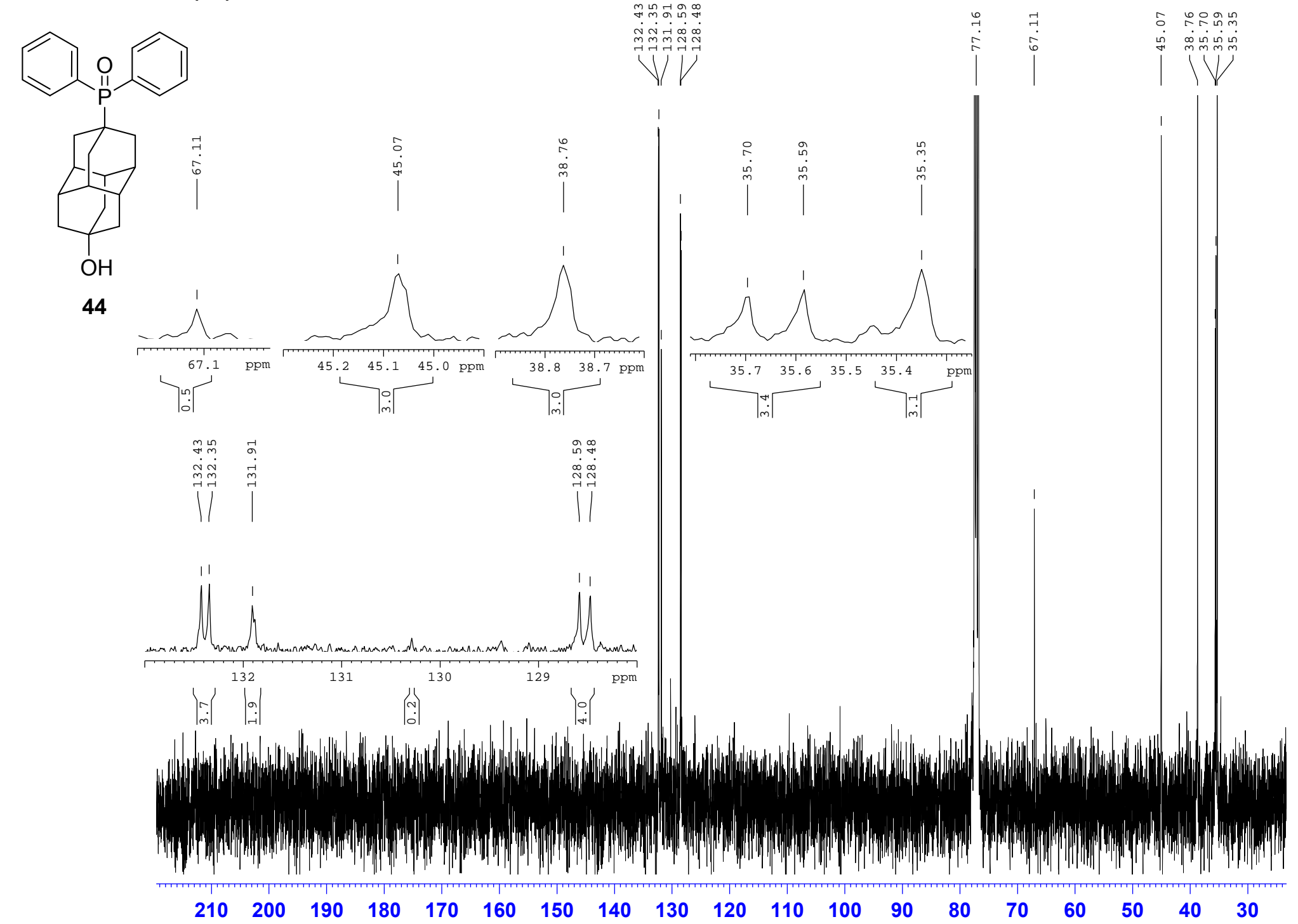


1.25.3. 9-OH-dia-4-P(=O) $\mathrm{Ph}_{2}{ }^{31} \mathrm{P}$

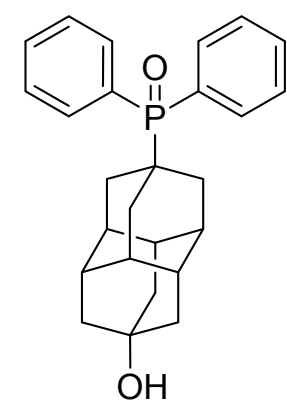

44

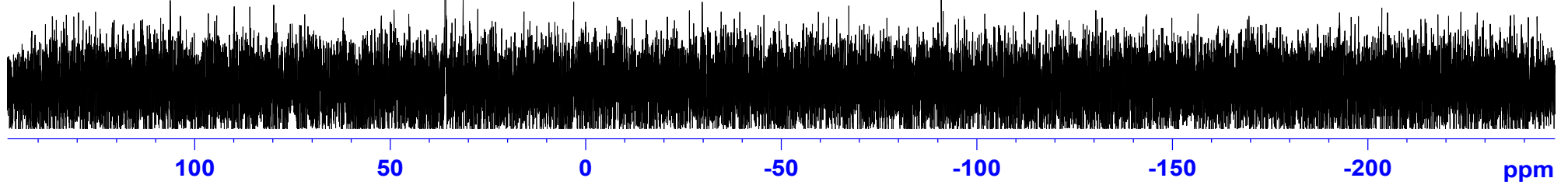


1.26. 1-Adamantyldiphenylphosphine sulphide (46)

1.26.1. 1-dia- $\mathrm{P}(=\mathrm{S}) \mathrm{Ph}_{2}{ }^{1} \mathrm{H}$

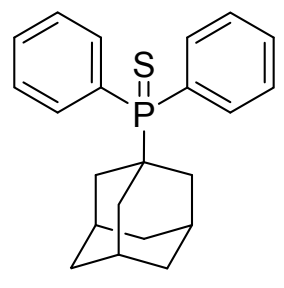

46 œ $\infty 0000000+N i n$ $\longrightarrow$

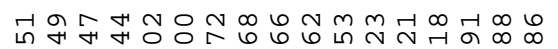

m m m ๓ N

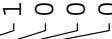

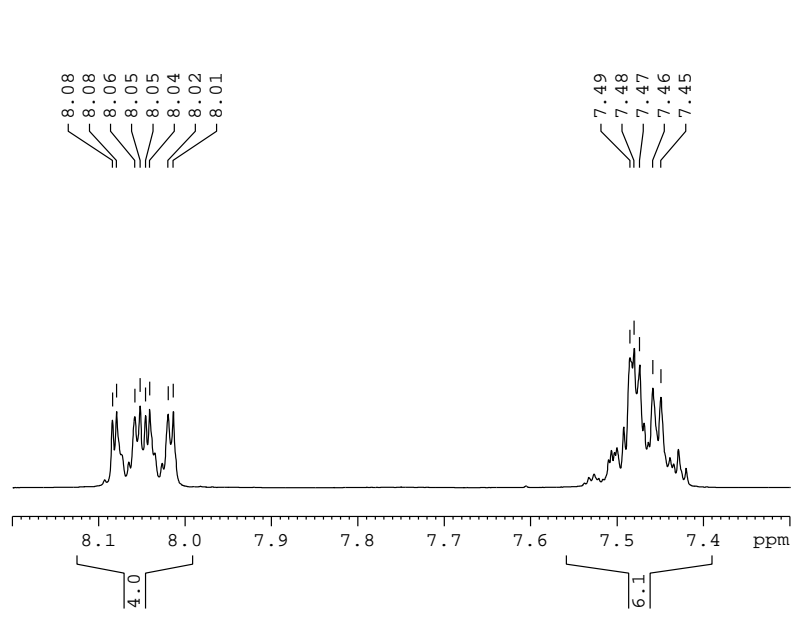
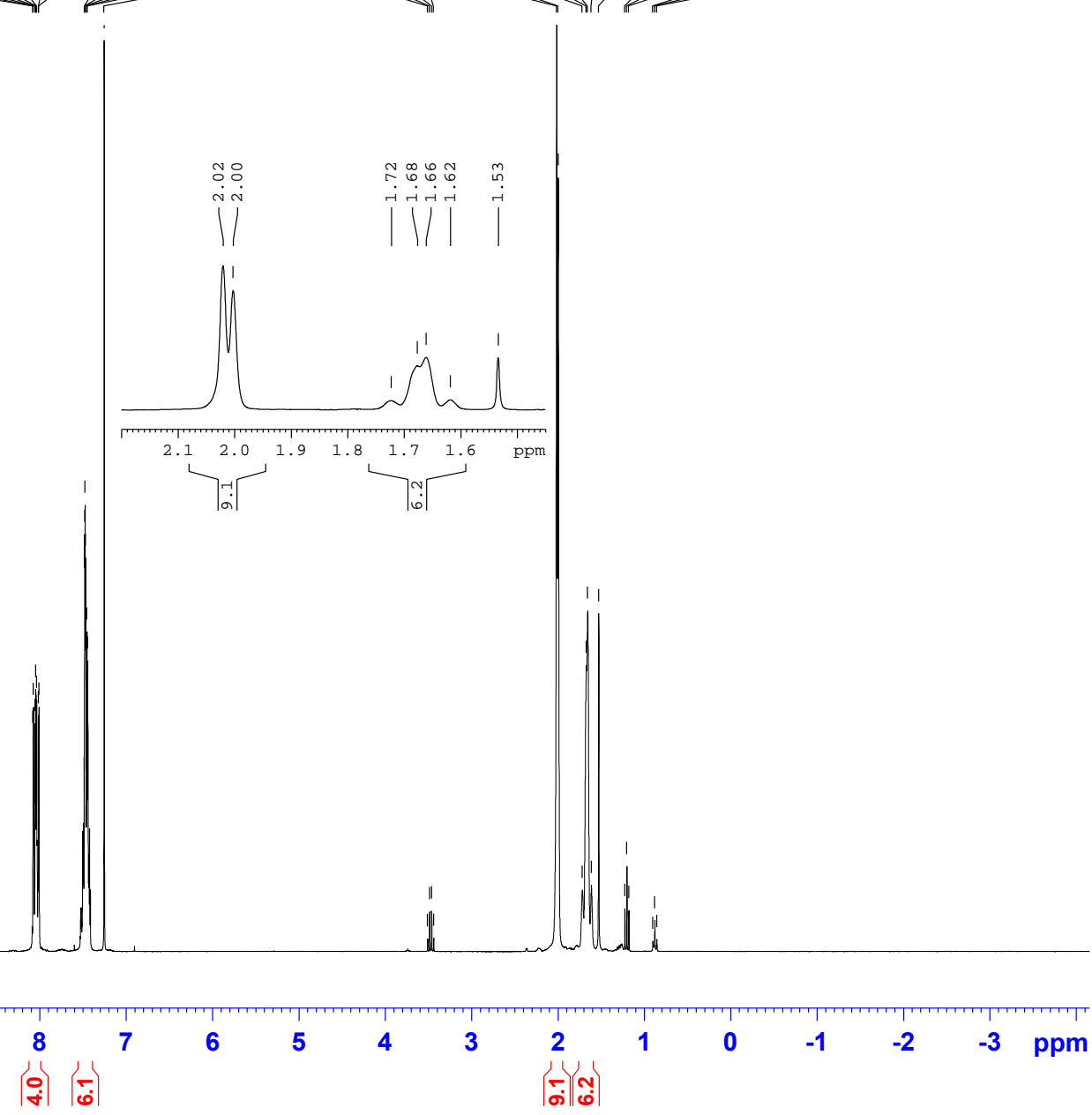
1.26.2. 1 -dia- $\mathrm{P}(=\mathrm{S}) \mathrm{Ph}_{2}{ }^{13} \mathrm{C}$

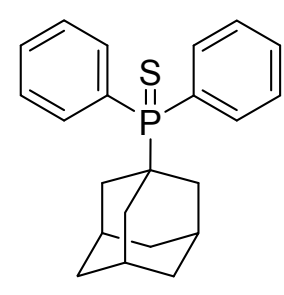

46
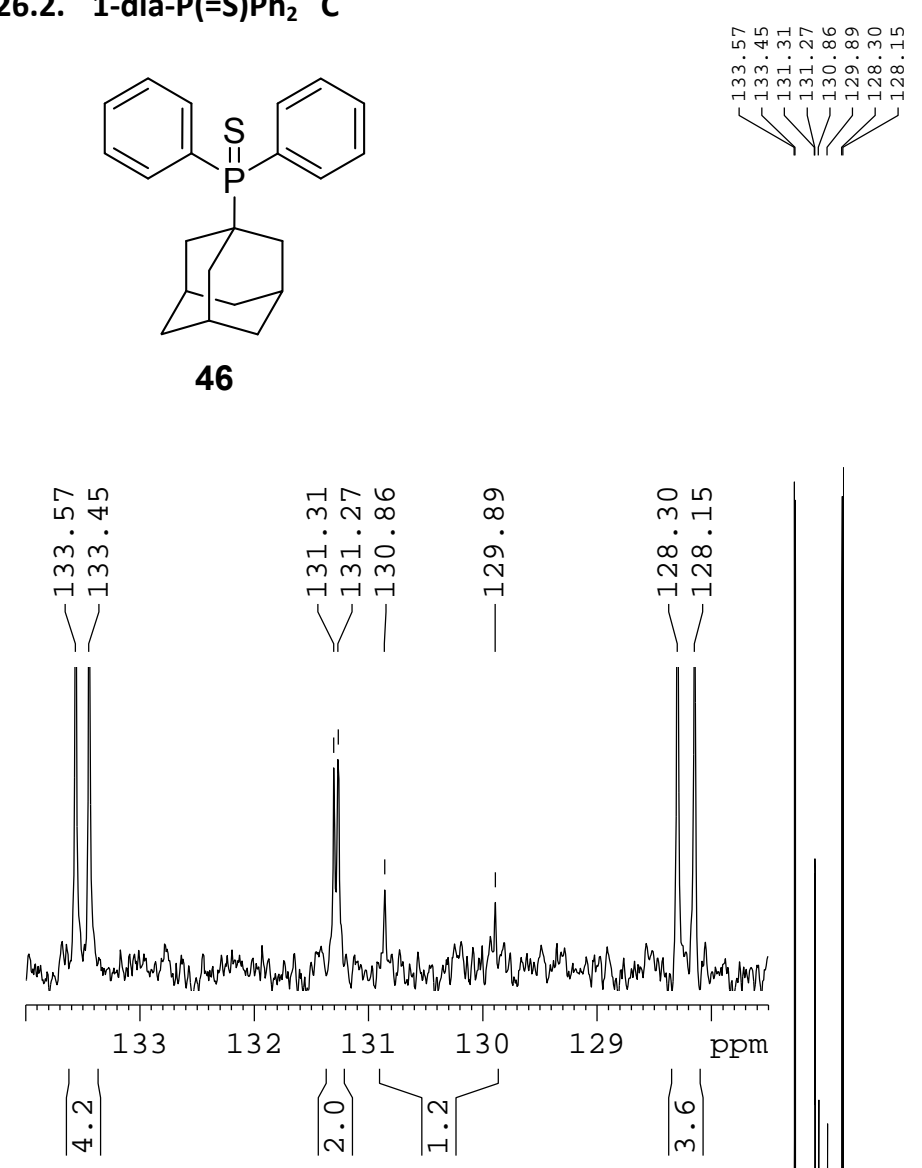

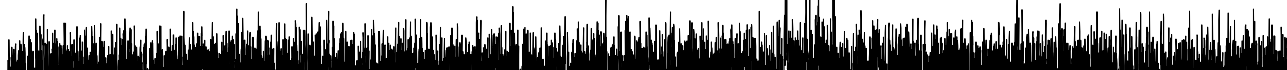

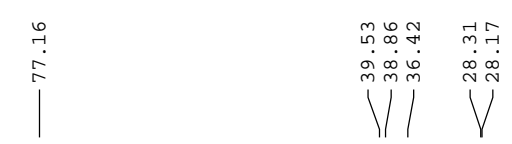

$\stackrel{n}{\infty} \bullet$

i

플ำ

$\stackrel{\infty}{\sim}$

$i \mid m$

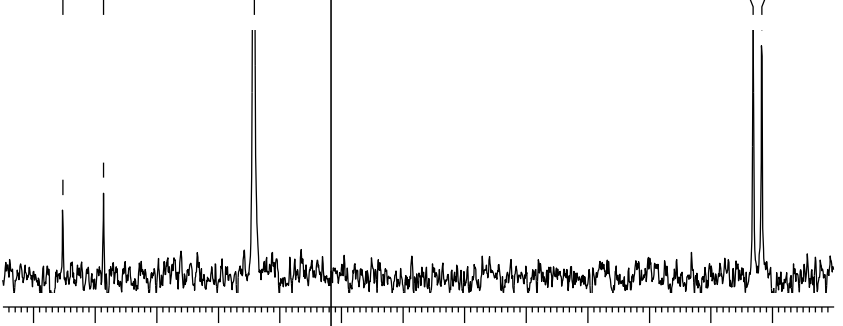

$\begin{array}{lllllllllllll}40 & 39 & 38 & 37 & 36 & 35 & 34 & 33 & 32 & 31 & 30 & 29 & \text { ppm }\end{array}$ î.

$\left|\begin{array}{l}0 \\ 0\end{array}\right|$

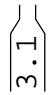

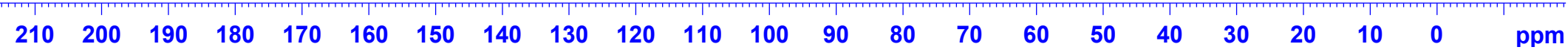


1.26.3. 1 dia- $\mathrm{P}(=\mathrm{S}) \mathrm{Ph}_{2}{ }^{31} \mathrm{P}$

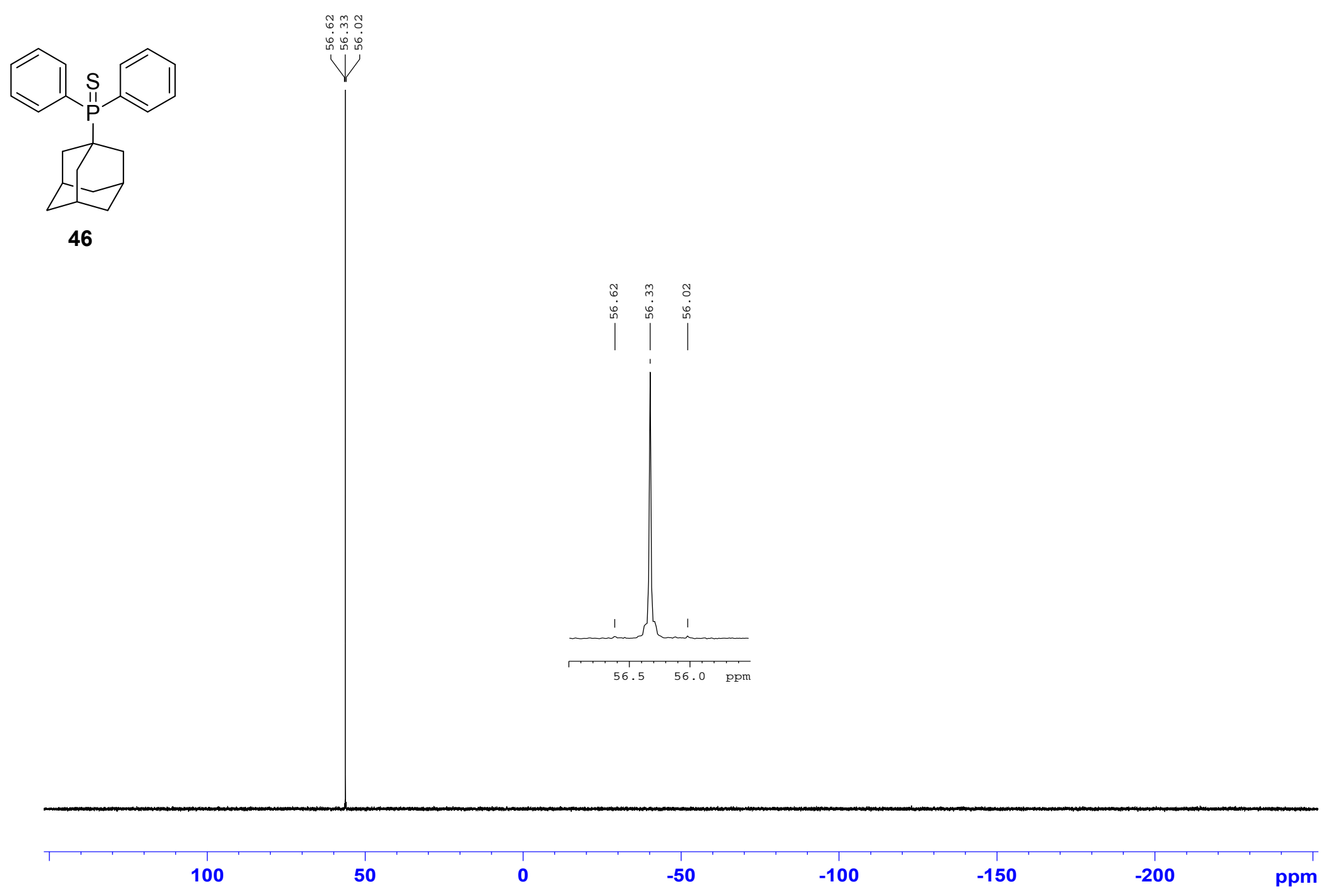


1.27. 1-Adamantyldiphenylphosphine selenide (47)

1.27.1. 1-dia-P(=Se) $\mathrm{Ph}_{2}{ }^{1} \mathrm{H}$

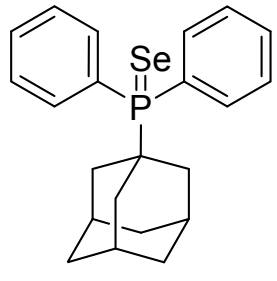

47

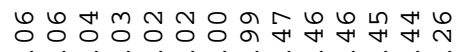
$\infty 0000000 \wedge+N R$

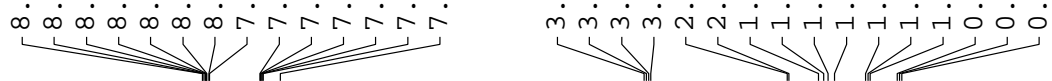

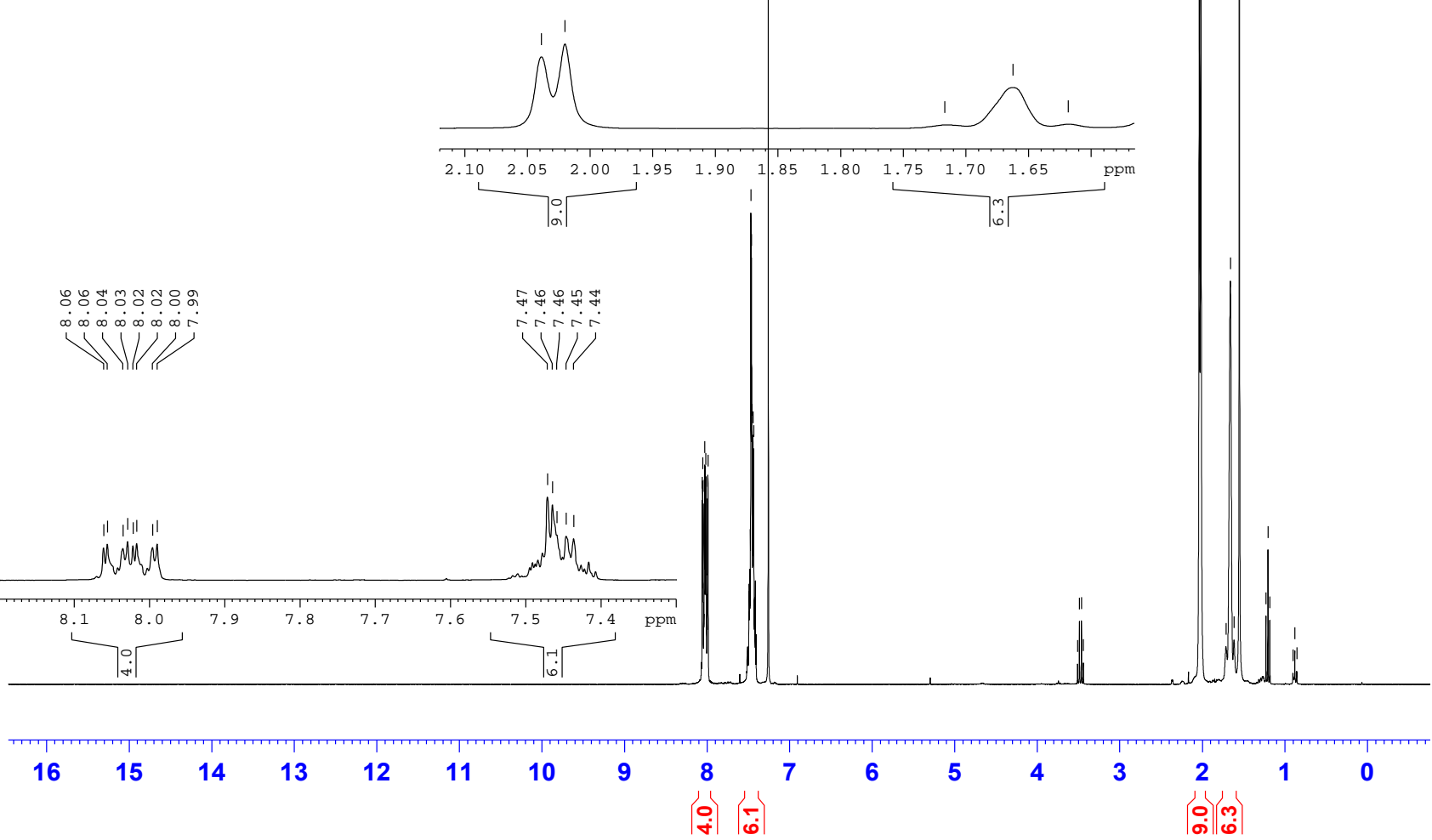

|

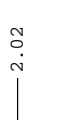


1.27.2. 1-dia- $\mathrm{P}(=\mathrm{Se}) \mathrm{Ph}_{2}{ }^{13} \mathrm{C}$

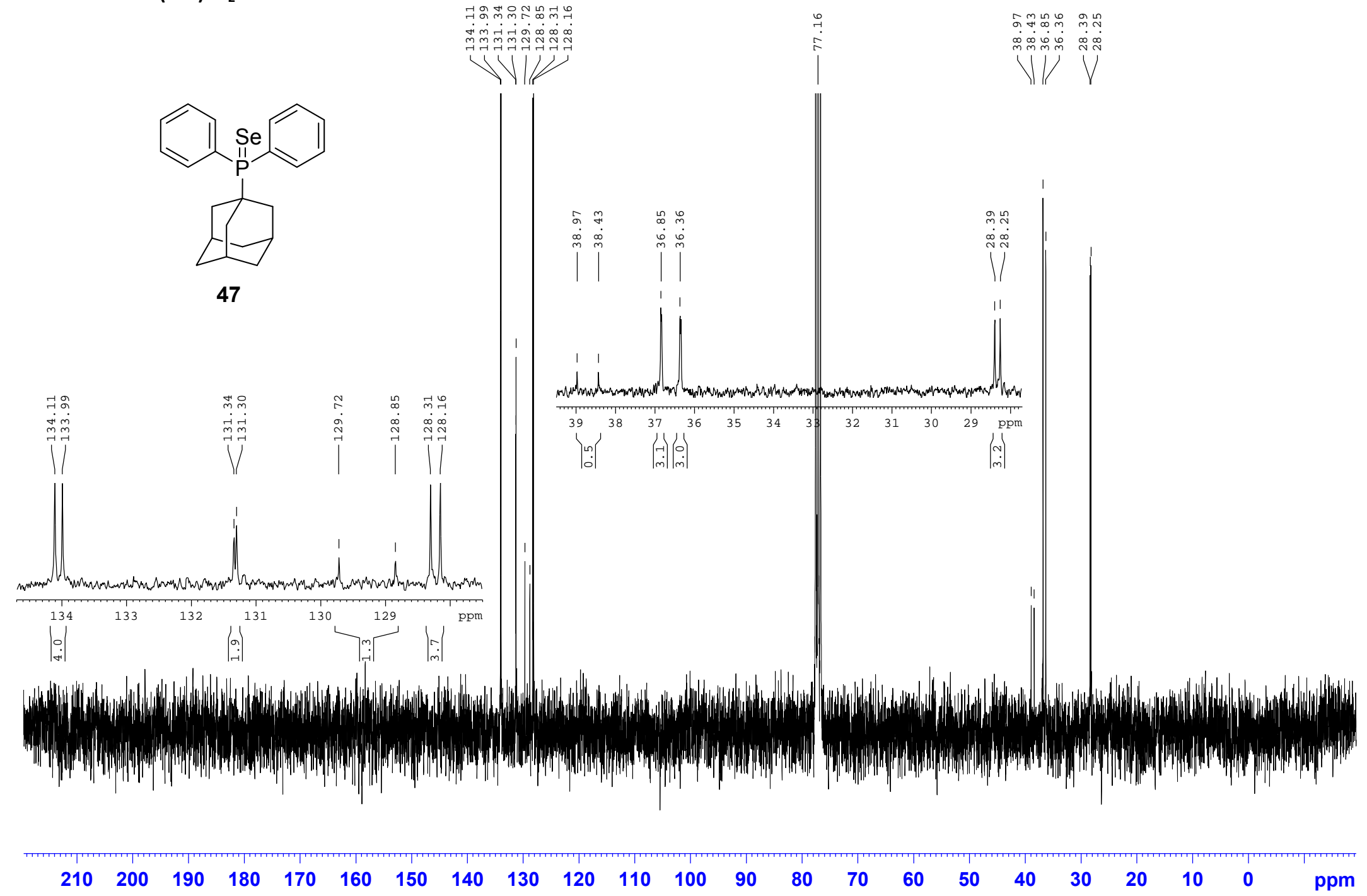


1.27.3. 1-Dia- $\mathrm{P}(=\mathrm{Se}) \mathrm{Ph}_{2}{ }^{31} \mathrm{P}$

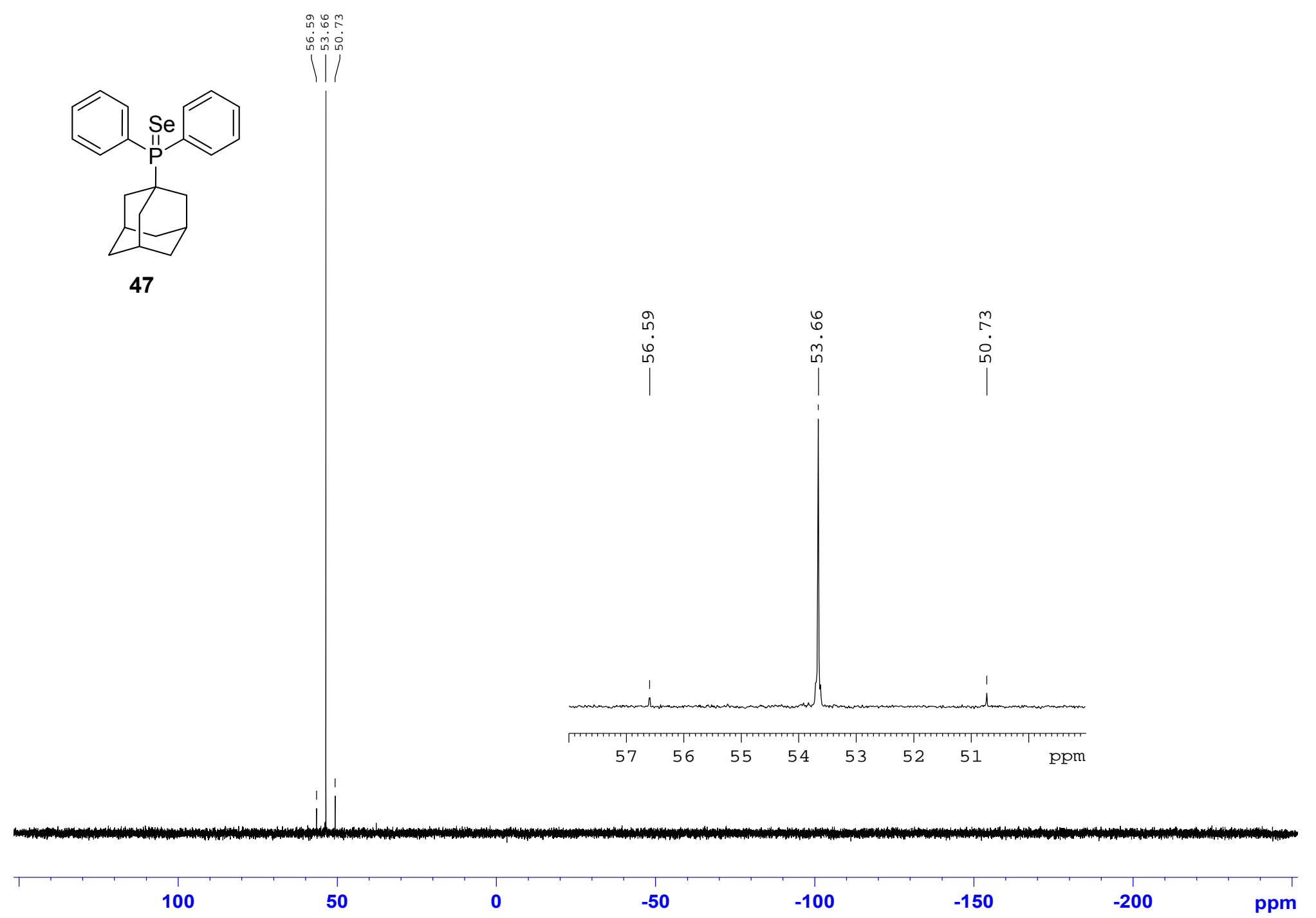


1.28. 1-Adamantyldiphenylphosphine (48)

1.28.1. 1-dia- $\mathrm{PPh}_{2}{ }^{1} \mathrm{H}$

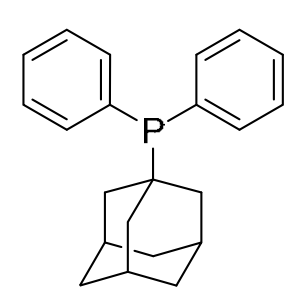

48

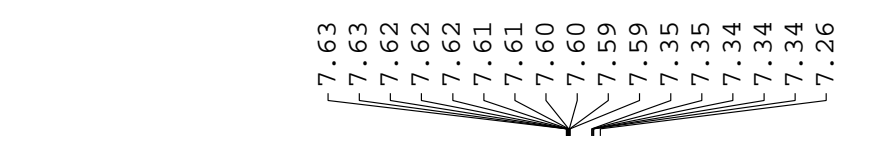

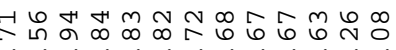

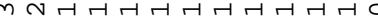

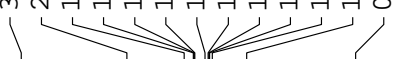

म

i

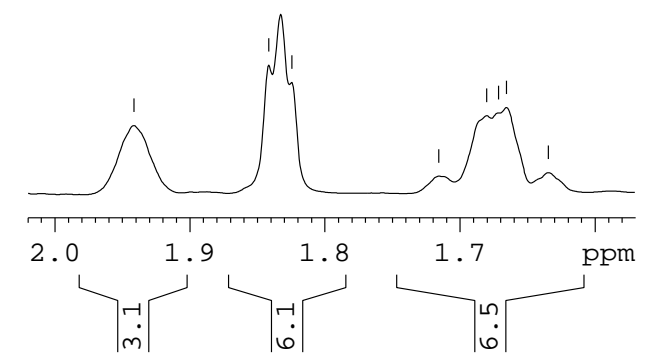

ชำำ ำ

rinitinina
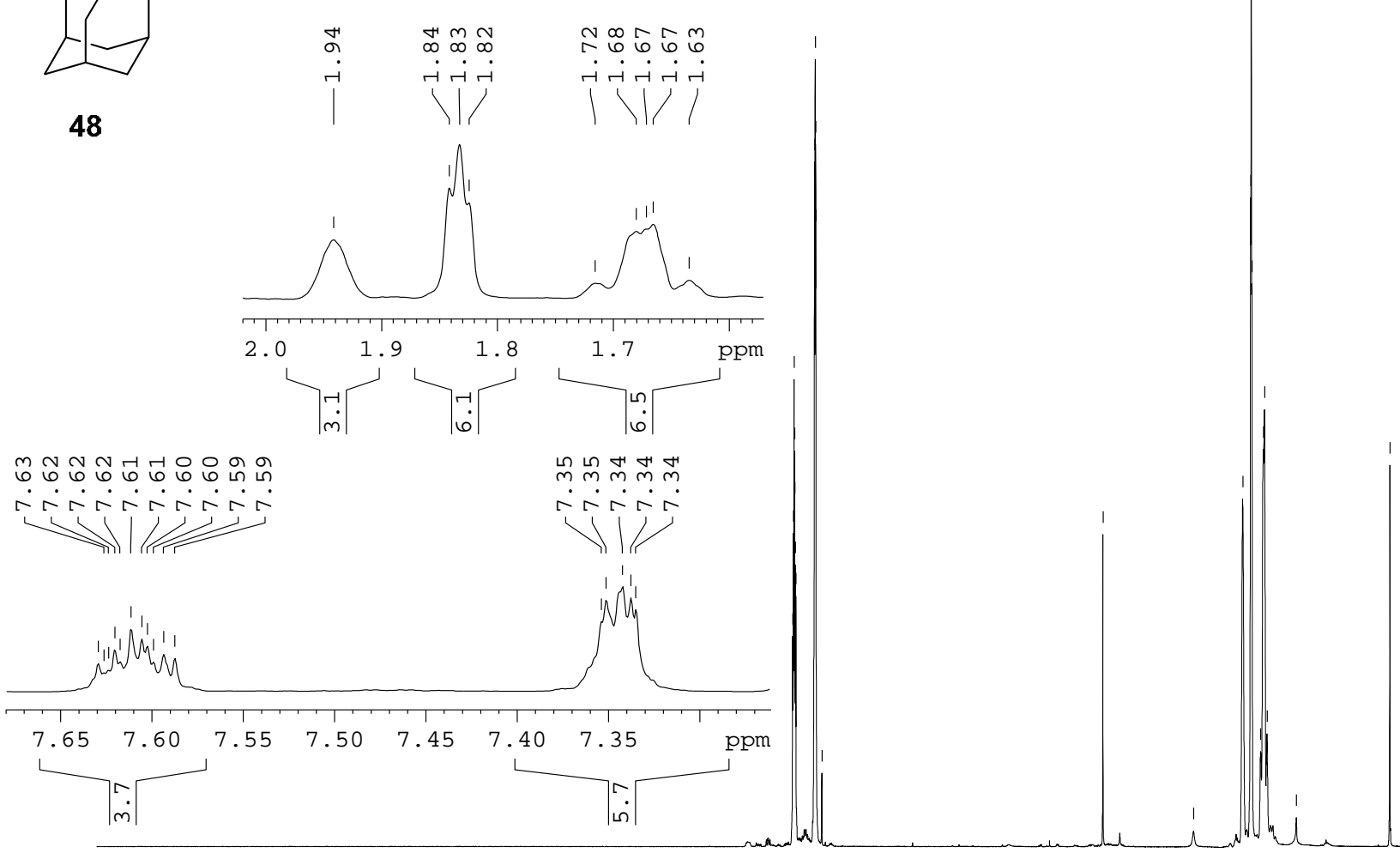

$\begin{array}{llllllll}16 & 15 & 14 & 13 & 12 & 11 & 10 & 9\end{array}$

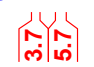

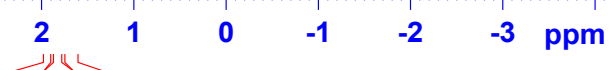


1.28.2. 1-dia- $\mathrm{PPh}_{2}{ }^{13} \mathrm{C}$
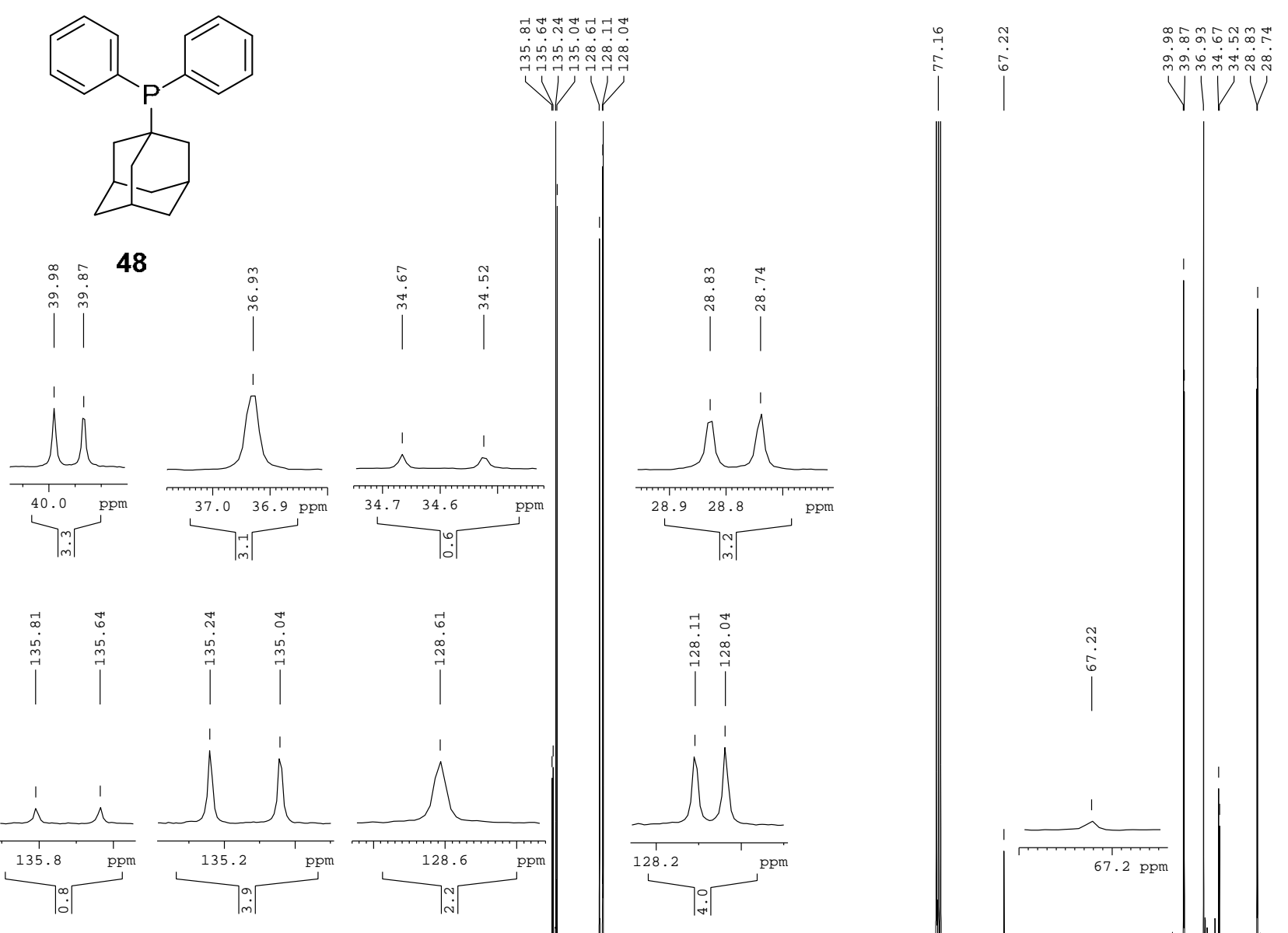

$\left.\right|^{\overrightarrow{0}}$

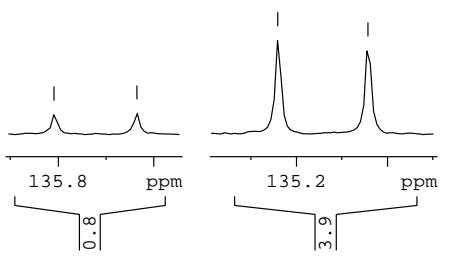

ลัก

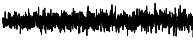

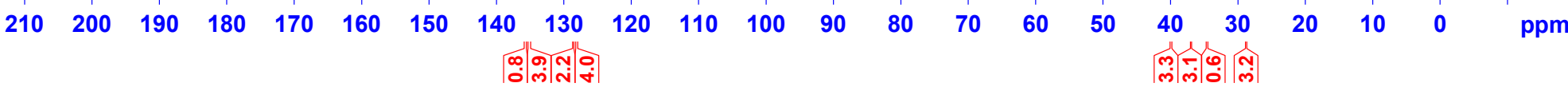


1.28.3. 1-dia- $\mathrm{PPh}_{2}{ }^{31} \mathrm{P}$<smiles>c1ccc(P(c2ccccc2)C23CC4CC(CC(C4)C2)C3)cc1</smiles>

48 
2. VT-NMR studies for primary phosphine $\mathbf{3 1}$ in different solvents
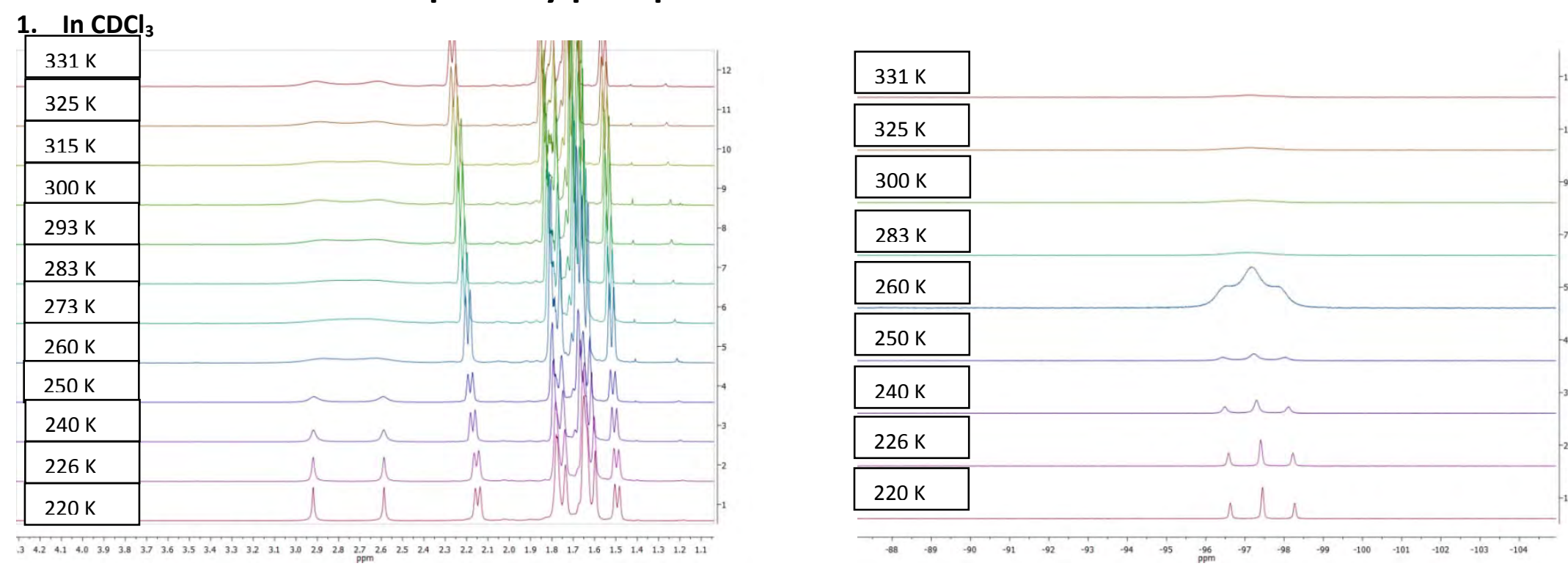

2. In $\mathrm{CD}_{2} \mathrm{Cl}_{2}$
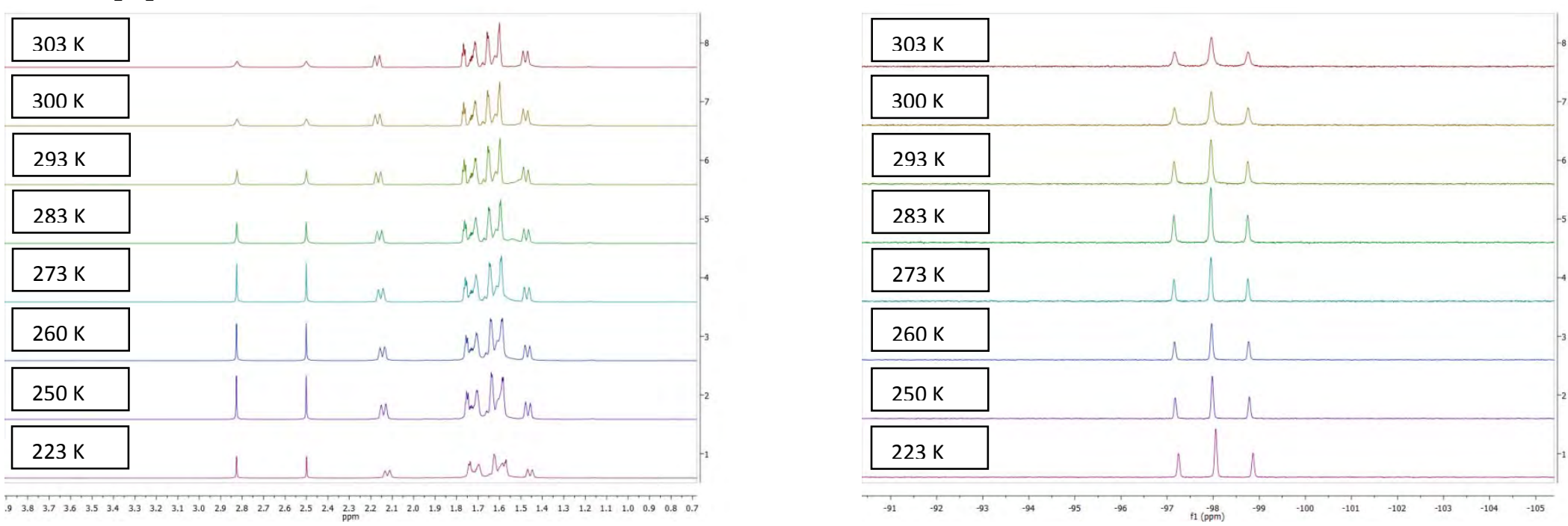


\section{In THF- ${ }^{8}$}

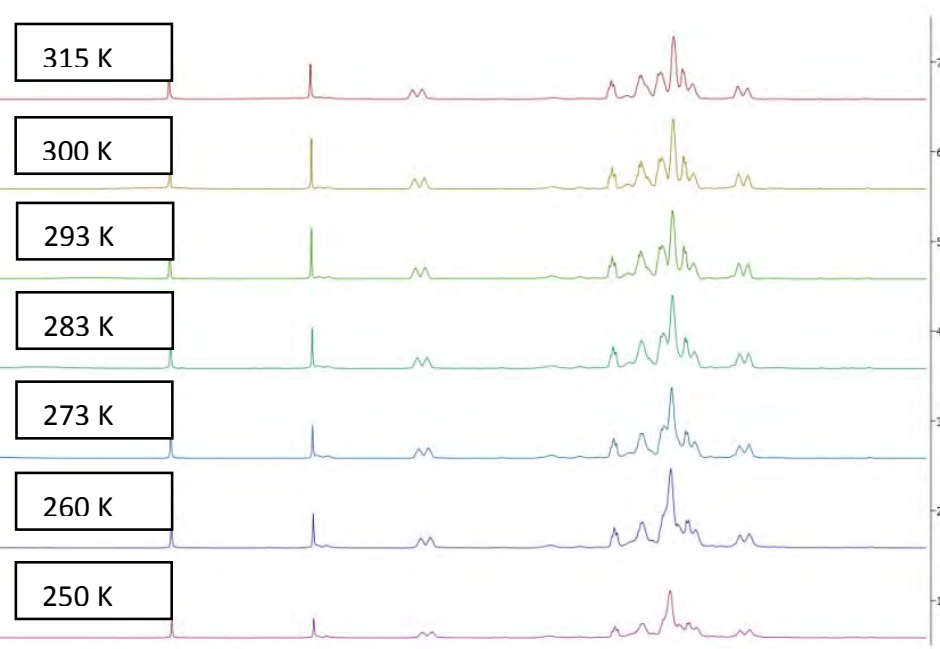

\begin{tabular}{lllllllllllllllllllllll}
1.2 & 3.1 & 1.0 & 2.9 & 2.8 & 2.7 & 2.6 & 2.5 & 2.4 & 2.3 & $\begin{array}{l}2.2 \\
2.2\end{array}$ & 2.1 & 2.0 & 1.9 & 1.8 & 1.7 & 1.6 & 1.5 & 1.4 & 1.3 & 1.2 \\
\hline
\end{tabular}

\section{In THF- $d^{8}+P h I$}

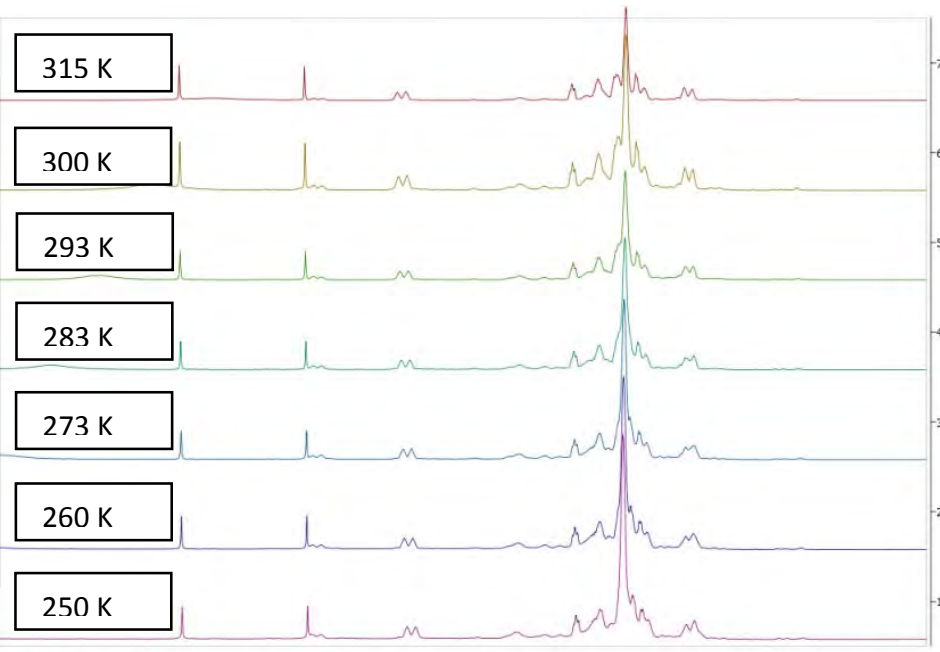

\begin{tabular}{lllllllllllllllllllllllllllll}
\hline 3 & 3.2 & 3.1 & 3.0 & 2.9 & 2.8 & 2.7 & 2.6 & 2.5 & 2.4 & 2.3 & 2.2 & 2.1 & 2.0 & 1.9 & 1.8 & 1.7 & 1.6 & 1.5 & 1.4 & 1.3 & 1.2 & 1.1 & 1.0
\end{tabular}
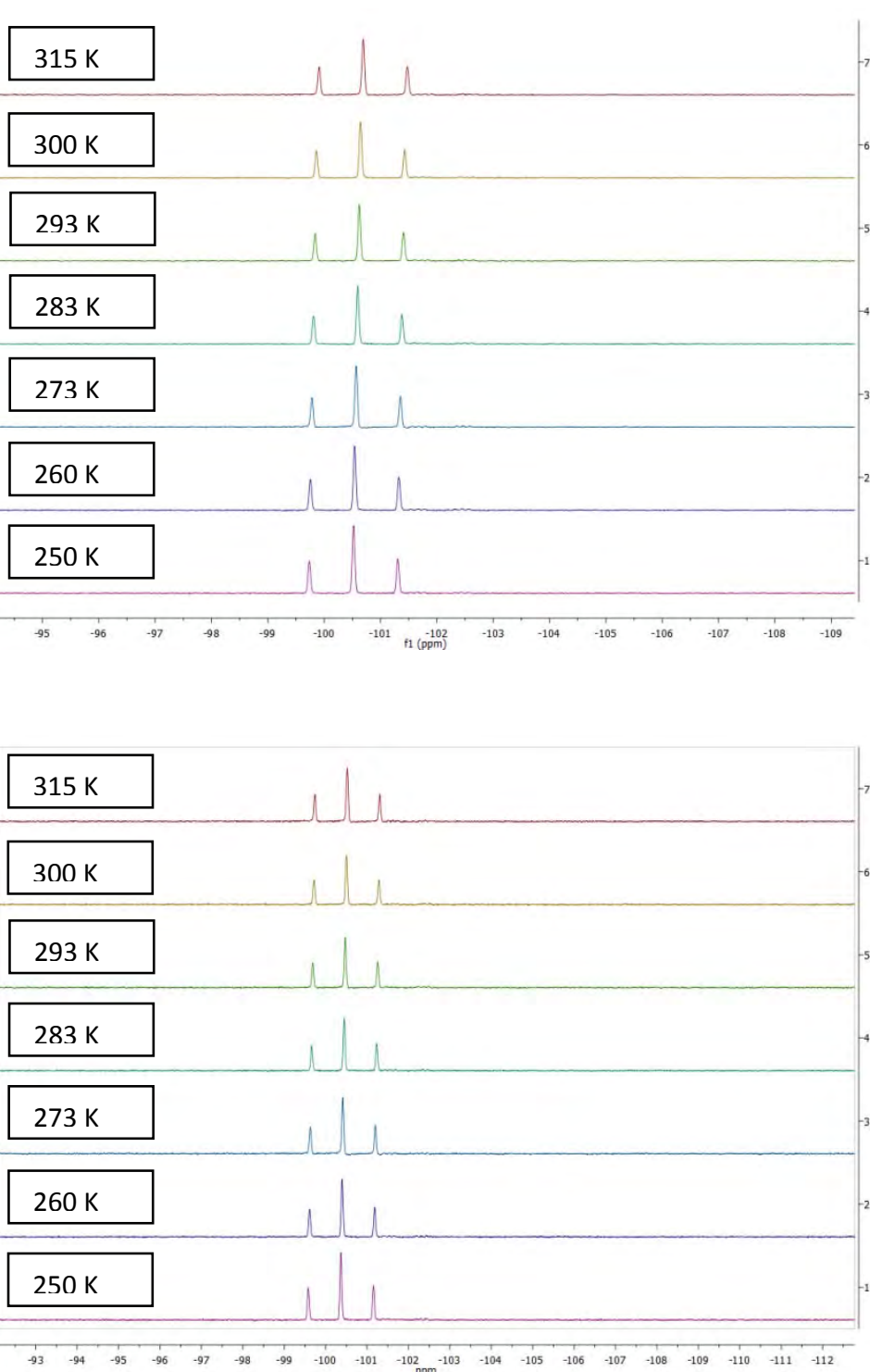


\section{In THF- $d^{8}+$ TBAI traces}

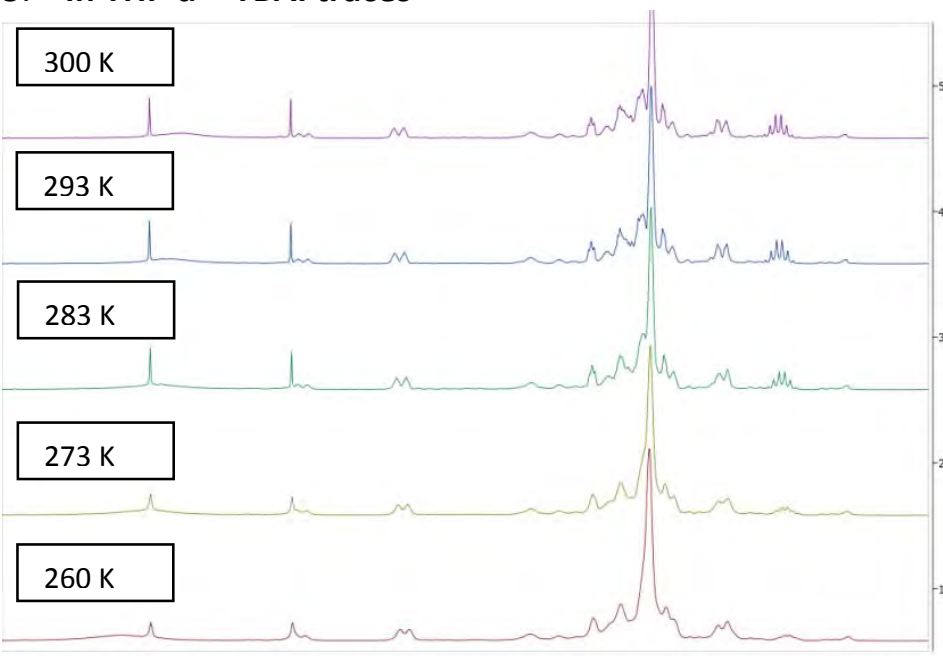

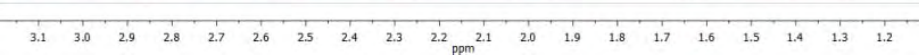

\section{In THF-d ${ }^{8}+\mathrm{HCl}$ concentrated}

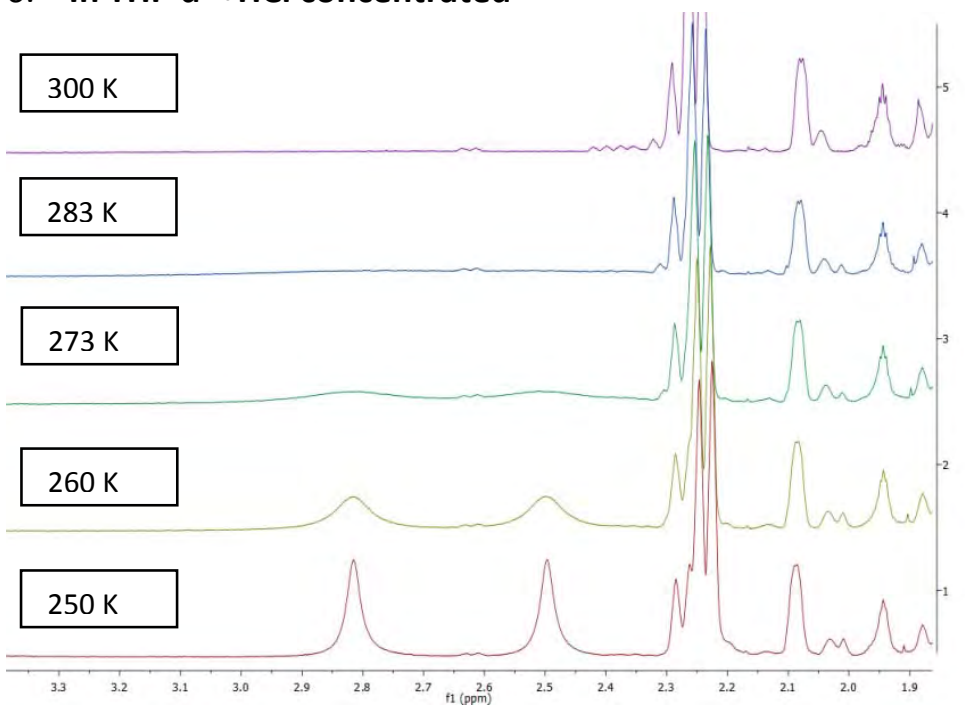

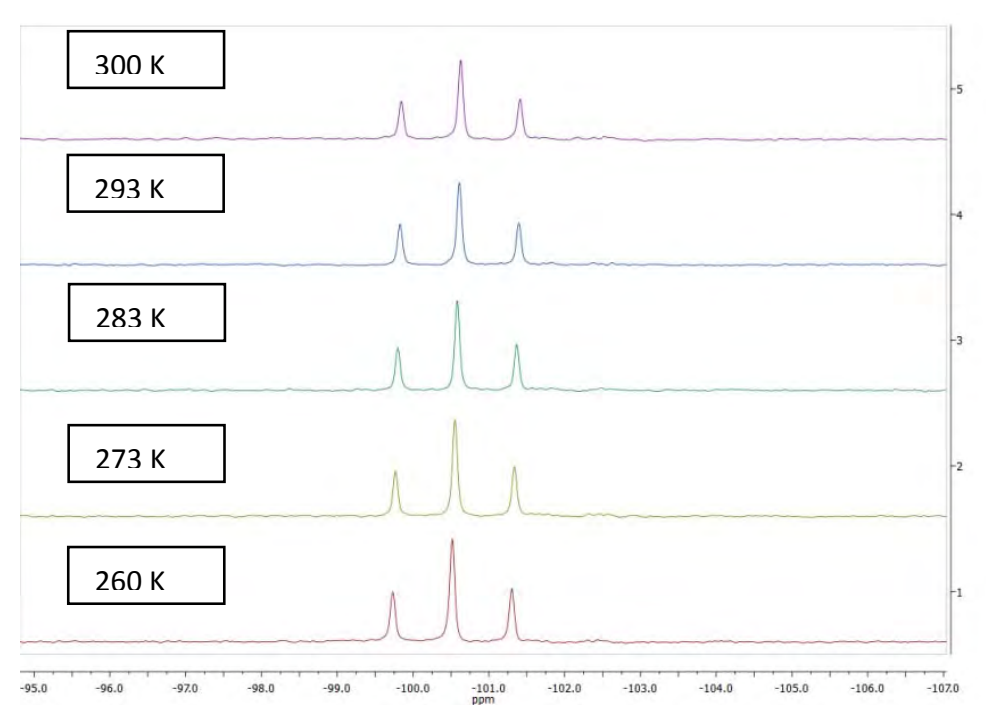

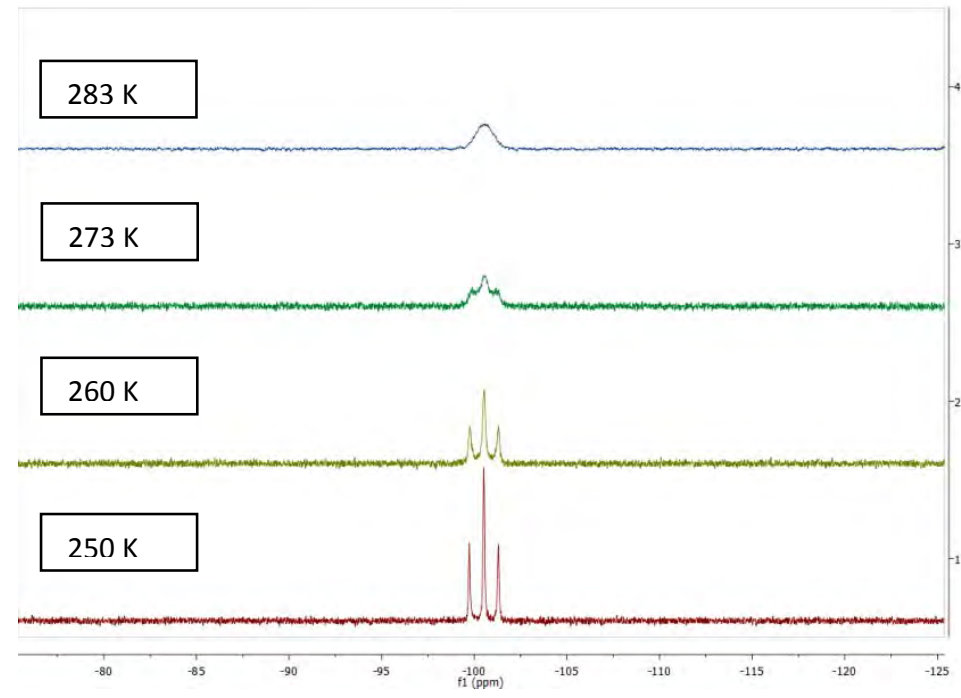




\section{In THF- $d^{8}+H I$ concentrated}
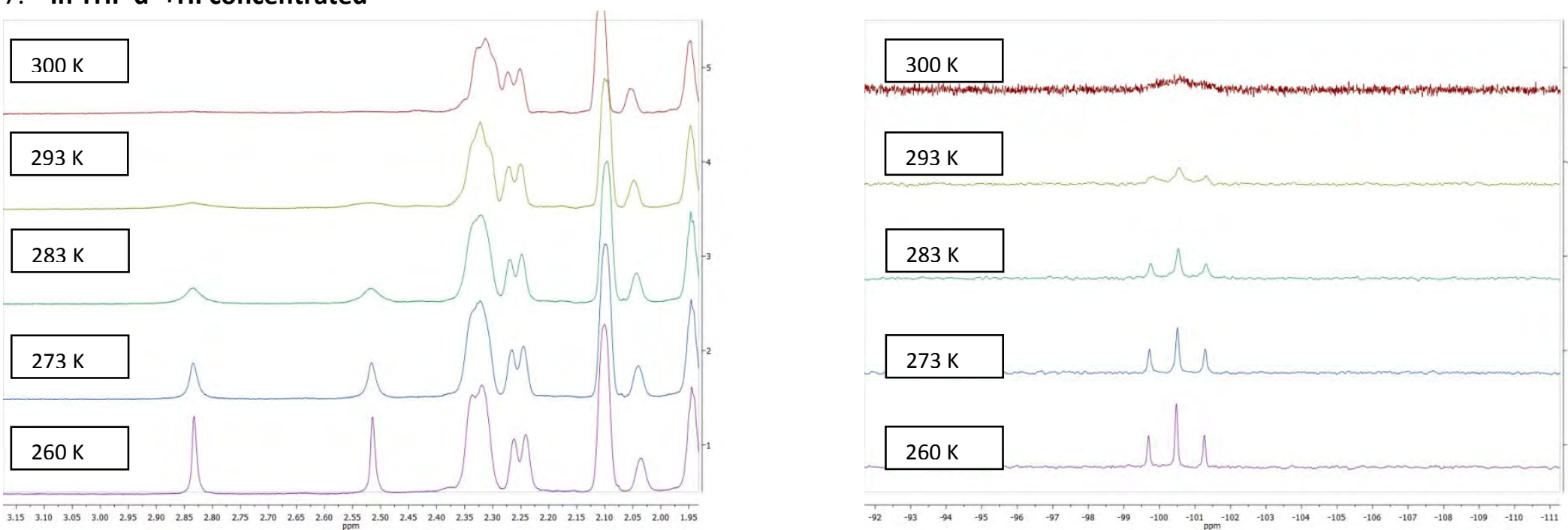

\section{In THF- $\mathrm{d}^{8}+\mathrm{H}_{2} \mathrm{SO}_{4}$ traces}
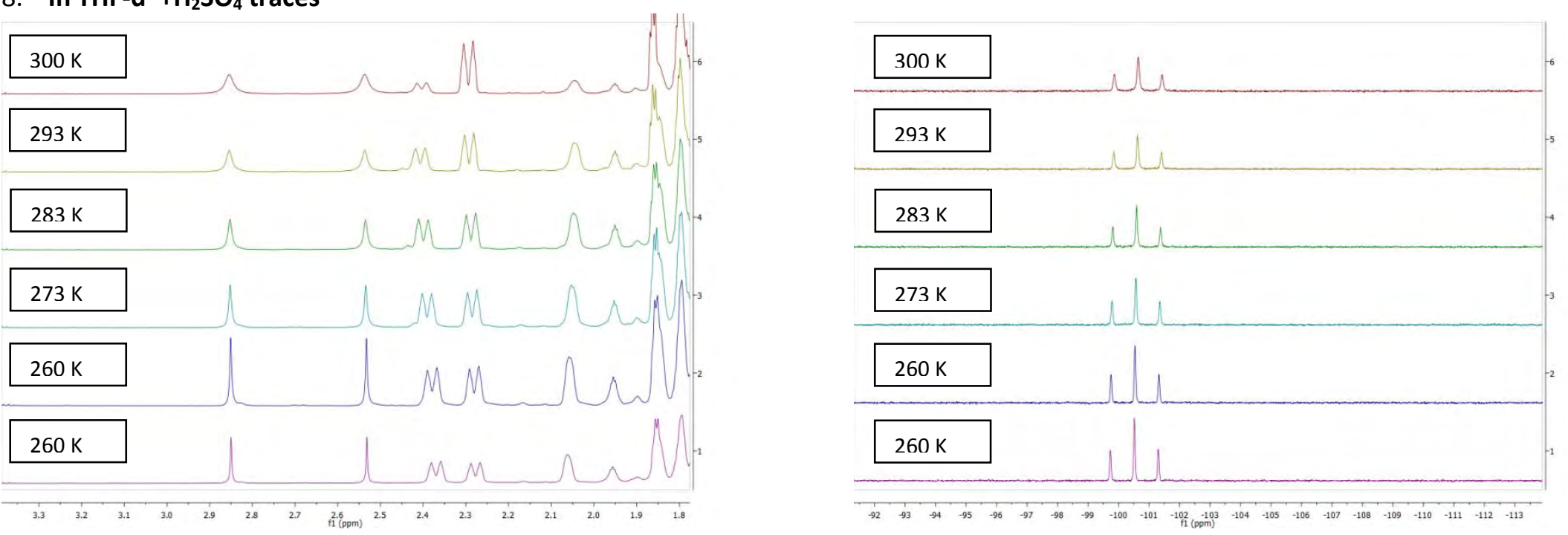


\section{In THF- $d^{8}+\mathrm{D}_{2} \mathrm{SO}_{4}$ traces}

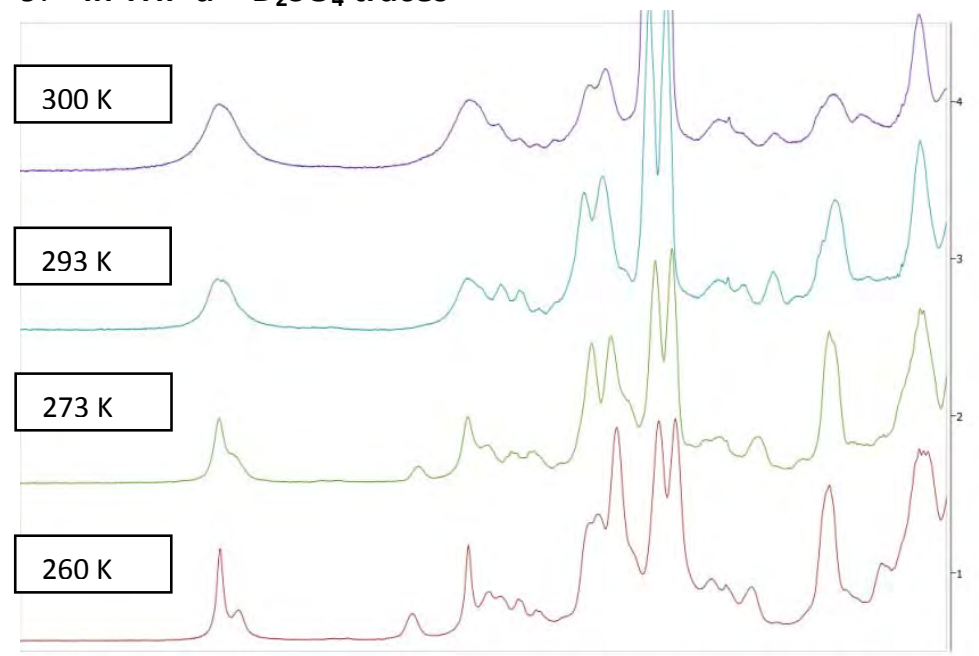

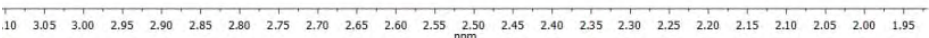

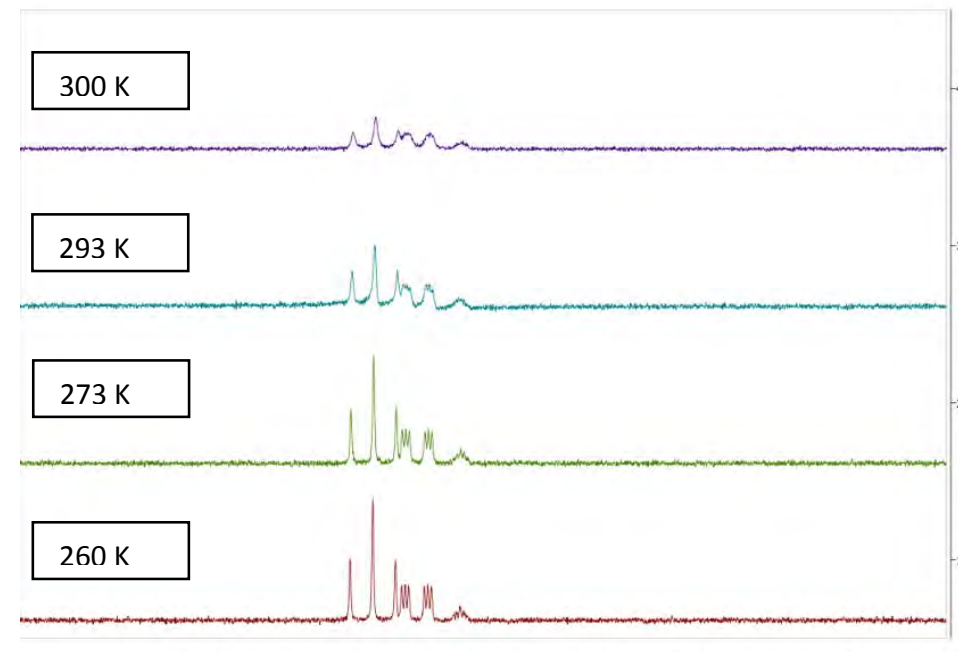

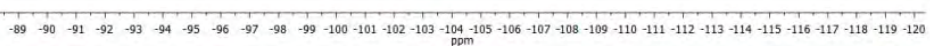




\section{Coordinates of diamondoid radical cations}<smiles>CCCCCCCCCCCCC</smiles>

$\begin{array}{lr}3^{\cdot+} & \\ C & -2.02580 \\ C & -1.31549 \\ \text { C } & -1.31549 \\ \text { C } & -0.62394 \\ \text { H } & -0.82350 \\ \text { H } & -2.34405 \\ \text { C } & -1.31549 \\ \text { H } & -3.06983 \\ \text { H } & -2.03905 \\ \text { C } & -1.31549 \\ \text { H } & -2.34405 \\ \text { H } & -0.82350 \\ \text { C } & 0.88646 \\ \text { H } & -0.56829 \\ \text { C } & 0.15104 \\ \text { H } & -1.80785 \\ \text { C } & 0.15104 \\ \text { H } & -1.80785 \\ \text { C } & 0.83813 \\ \text { H } & 0.16687 \\ \text { H } & 0.66170 \\ \text { H } & 1.38018 \\ \text { H } & 1.38018 \\ \text { P } & 2.66645 \\ \text { H } & 0.66170 \\ \text { H } & 0.16687 \\ \text { H } & 3.26191 \\ \text { H } & 3.26191\end{array}$

$-0.90529$

$-0.37856$

1.15732

1.67439

1.54334

1.52967

$-0.37856$

$-0.58182$

$-1.99903$

1.15732

1.52967

1.54334

1. 20151

2.76768

$-0.87979$

$-0.75731$

$-0.87979$

$-0.75731$

$-0.35638$

$-1.97186$

$-0.52551$

1.59937

1.59937

$-0.75357$

$-0.52551$

$-1.97186$

$-0.09587$

$-0.09587$
0.00000

1. 25712

1.26120

0.00000

2. 15867

1. 28566

$-1.25712$

0.00000

0.00000

$-1.26120$

$-1.28566$

$-2.15867$

$\odot .0000 \odot$

0.00000

$-1.26663$

$-2.15506$

1. 26663

2.15506

0.00000

1. 28312

2.16675

0.88865

$-0.88865$

0.00000

$-2.16675$

$-1.28312$

1. 09681

$-1.09681$<smiles></smiles>

$\begin{array}{lr}\mathbf{3 4}^{\cdot+} & \\ \mathrm{C} & -1.19287 \\ \mathrm{C} & -0.81671 \\ \mathrm{C} & -1.56302 \\ \mathrm{C} & -1.23206 \\ \mathrm{H} & -1.30796 \\ \mathrm{H} & -2.64454 \\ \mathrm{C} & -0.80504 \\ \mathrm{H} & -2.26548 \\ \mathrm{H} & -0.68439 \\ \mathrm{C} & -1.54654 \\ \mathrm{H} & -2.62852 \\ \mathrm{H} & -1.28841 \\ \mathrm{C} & 0.38908 \\ \text { O } & -1.83236 \\ \mathrm{C} & 0.72038 \\ \mathrm{H} & -1.06127 \\ \mathrm{C} & 0.70817 \\ \mathrm{H} & -1.08055\end{array}$

$-1.94850$

$-1.13281$

๑. 20969

$\odot .98513$

0.80317

0.04510

$-1.16264$

$-2.15786$

$-2.91592$

0.17955

0.02282

0.76275

1. 26526

2. 22885

$-0.91406$

$-1.73371$

$-0.88044$

$-1.68200$
0.01748

1.26540

1. 25625

$-0.01911$

2.14134

1.27967

$-1.24581$

0.01479

0.03351

$-1.27764$

$-1.30787$

$-2.16499$

$-0.01655$

$-0.11154$

$-1.25123$

$-2.14063$

1. 27792

2. 17169 


$\begin{array}{rrrr}\mathrm{C} & 1.06879 & -0.10516 & 0.00445 \\ \mathrm{H} & 1.24195 & -1.83251 & 1.30573 \\ \mathrm{H} & 0.99025 & -0.31858 & 2.17312 \\ \mathrm{H} & 0.59966 & 1.86732 & 0.86829 \\ \mathrm{H} & 0.59226 & 1.84720 & -0.91474 \\ \mathrm{P} & 2.89599 & 0.37059 & 0.00243 \\ \mathrm{H} & 1.01011 & -0.37552 & -2.15788 \\ \mathrm{H} & 1.25466 & -1.86639 & -1.24777 \\ \mathrm{H} & 3.03606 & 1.28976 & 1.06655 \\ \mathrm{H} & 3.04067 & 1.25219 & -1.09216 \\ \mathrm{H} & -1.96655 & 2.62642 & 0.76024\end{array}$<smiles>PC12CC3CC(C1)CC(P)(C3)C2</smiles>

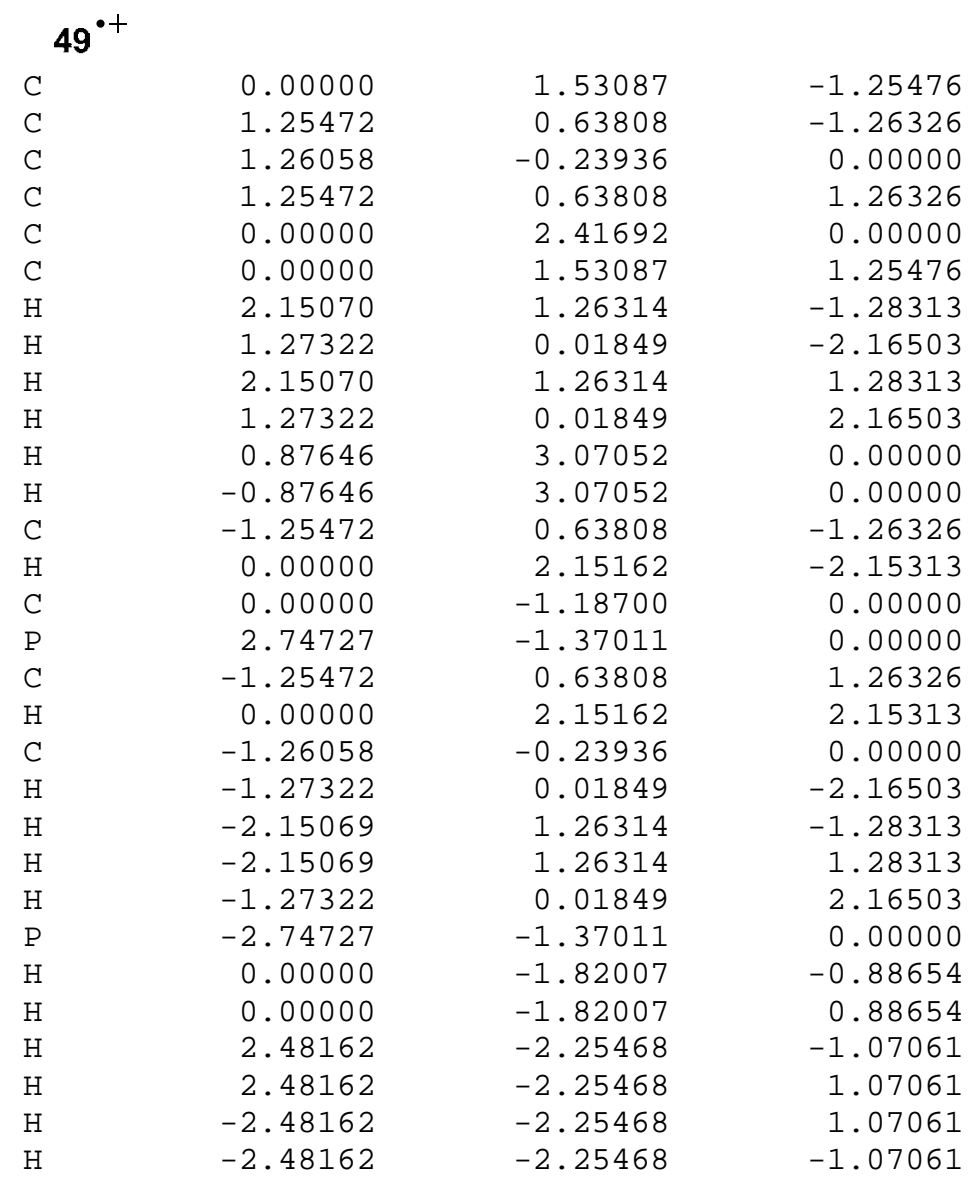

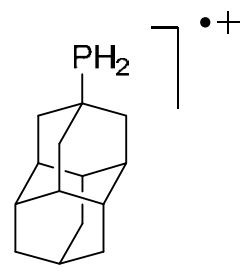

$\begin{array}{lrrr} & & & \\ \mathrm{C2}^{\mathbf{0}^{+}} & & & \\ \mathrm{C} & 0.90463 & 0.000 \odot \odot & 1.45159 \\ \mathrm{C} & 0.33952 & -1.25116 & 0.75007 \\ \mathrm{C} & 0.79907 & -1.25533 & -0.71990 \\ \mathrm{H} & 0.25432 & 0.00000 & -1.40445 \\ & 0.42284 & -2.15038 & -1.22631\end{array}$


2. 15038

0.00000

$-1.22631$

0.00000

$-1.36555$

1. 25994

$-2.47267$

0.82097

2. 15244

1.25287

0.82097

$-1.25994$

1.25287

$-2.15244$

0.12782

1.86644

$-1.28002$

0.33948

$-2.16262$

$-1.89060$

0.88818

0.00000

$-1.89060$

0.02587

0.33948

2. 16262

1.86644

$-1.08221$

1. 08221

$0.0000 \odot$

$-0.87806$

0.87806

2. 16021

1. 27554

0.00000

$-1.27554$

$-2.16021$

$-0.81702$

$-0.81702$

$-0.09310$

1.89502

1.89502

$-0.32416$

$-1.84142$

$-0.14621$

$-1.84142$

$-0.32416$<smiles>C[CH+]CP</smiles>

$\begin{array}{lrrr} & 36^{\circ+} & & \\ \mathrm{C} & -0.61623 & -0.41775 & 1.41011 \\ \mathrm{C} & -0.05172 & 0.98344 & 1.08837 \\ \mathrm{C} & -0.53041 & 1.40297 & -0.31579 \\ \mathrm{C} & 0.01590 & 0.39469 & -1.33594 \\ \mathrm{H} & -0.15672 & 2.40520 & -0.54966 \\ \mathrm{C} & -2.06944 & 1.41584 & -0.37583 \\ \mathrm{C} & -0.05375 & -1.41827 & 0.37651 \\ \mathrm{C} & -2.15193 & -0.39539 & 1.34733 \\ \mathrm{H} & -0.29912 & -0.71583 & 2.41478 \\ \mathrm{C} & -0.53048 & -1.00463 & -1.02942 \\ \mathrm{C} & -2.07286 & -0.97700 & -1.08874 \\ \mathrm{H} & -0.15958 & -1.71895 & -1.77233 \\ \mathrm{C} & 1.59630 & 0.39005 & -1.31847 \\ \mathrm{H} & -0.24553 & 0.69632 & -2.35643 \\ \mathrm{C} & 1.49067 & -1.44526 & 0.42832 \\ \mathrm{H} & -0.41382 & -2.42515 & 0.60494 \\ \mathrm{C} & 1.49206 & 0.97497 & 1.14713 \\ \mathrm{H} & -0.41142 & 1.70315 & 1.82847 \\ \mathrm{C} & 2.00344 & -0.03660 & 0.11797 \\ \mathrm{H} & 1.82051 & 0.69483 & 2.15085 \\ \mathrm{H} & 1.87794 & 1.97754 & 0.93988 \\ \mathrm{H} & 1.95091 & 1.39180 & -1.56926\end{array}$




$\begin{array}{lrrr}H & 1.95331 & -0.31086 & -2.07571 \\ \mathrm{P} & 3.86888 & 0.00204 & -0.01217 \\ \mathrm{H} & 1.87486 & -2.17255 & -0.29315 \\ \mathrm{H} & 1.81920 & -1.75941 & 1.42182 \\ \mathrm{H} & 4.24449 & 1.28123 & -0.47382 \\ \mathrm{H} & 4.24564 & -0.81855 & -1.09634 \\ \mathrm{C} & -2.61846 & 0.02294 & -0.05546 \\ \mathrm{H} & -2.55545 & 0.30244 & 2.08528 \\ \mathrm{H} & -2.54962 & -1.38622 & 1.59167 \\ \mathrm{H} & -2.46229 & -1.98053 & -0.88878 \\ \mathrm{H} & -2.41641 & -0.69082 & -2.08586 \\ \mathrm{O} & -4.03087 & 0.12726 & -0.13777 \\ \mathrm{H} & -2.41684 & 1.72305 & -1.36531 \\ \mathrm{H} & -2.46539 & 2.13930 & 0.34088 \\ \mathrm{H} & -4.43953 & -0.72460 & 0.05627\end{array}$<smiles>CC[CH-]CP</smiles>

\begin{tabular}{|c|c|c|c|}
\hline C & 0.72169 & $-\odot .26992$ & 1.28133 \\
\hline C & $-\odot .72169$ & ๑.26992 & 1.28133 \\
\hline C & -1.44575 & -0.25874 & 0.02888 \\
\hline C & -0.71711 & 0.26244 & -1.21768 \\
\hline H & -2.48337 & ๑. 08861 & 0.02563 \\
\hline C & -1.45738 & -1.80818 & $\odot .02757$ \\
\hline C & 1.44575 & 0.25874 & ๑. 02888 \\
\hline C & $\odot .71711$ & -1.81476 & 1.28479 \\
\hline $\mathrm{H}$ & 1.24301 & ๑. 07280 & 2.18024 \\
\hline C & $\odot .71711$ & -0.26244 & -1.21768 \\
\hline C & 0.71312 & -1.83081 & -1.22974 \\
\hline $\mathrm{H}$ & 1.24052 & 0.05633 & -2.12525 \\
\hline C & -0.71312 & 1.83081 & -1.22974 \\
\hline $\mathrm{H}$ & -1.24052 & $-\odot .05633$ & -2.12525 \\
\hline C & 1.45738 & 1.80818 & 0.02757 \\
\hline $\mathrm{H}$ & 2.48337 & $-\odot .08861$ & $\odot .02563$ \\
\hline C & -0.71711 & 1.81476 & 1.28479 \\
\hline $\mathrm{H}$ & -1.24301 & $-\odot .07280$ & 2.18024 \\
\hline C & 0.01326 & 2.31948 & $\odot .03384$ \\
\hline H & $-\odot .22316$ & 2.17665 & 2.19014 \\
\hline $\mathrm{H}$ & -1.74657 & 2.18410 & 1.30865 \\
\hline $\mathrm{H}$ & -1.74672 & 2.18313 & -1.26221 \\
\hline H & -0.21413 & 2.17983 & -2.13625 \\
\hline $\mathrm{P}$ & -0.00382 & 4.21542 & ๑. 00477 \\
\hline H & 1.99566 & 2.17013 & -0.85308 \\
\hline H & 1.99921 & 2.16325 & 0.90762 \\
\hline H & -1.36902 & 4.47885 & -0.26273 \\
\hline H & 0.48218 & 4.48289 & -1.29807 \\
\hline C & -0.01326 & -2.31948 & 0.03384 \\
\hline $\mathrm{H}$ & 0.22316 & -2.17665 & 2.19014 \\
\hline H & 1.74657 & -2.18410 & 1.30865 \\
\hline $\mathrm{H}$ & 1.74672 & -2.18313 & -1.26221 \\
\hline $\mathrm{H}$ & 0.21413 & -2.17983 & -2.13625 \\
\hline$P$ & $\odot . \odot \odot 382$ & -4.21542 & $\odot .0 \odot 477$ \\
\hline H & -1.99566 & -2.17013 & -0.85308 \\
\hline $\mathrm{H}$ & -1.99921 & -2.16325 & 0.90762 \\
\hline H & 1.36902 & -4.47885 & $-\odot .26273$ \\
\hline H & -0.48218 & -4.48289 & -1.29807 \\
\hline
\end{tabular}


Table S1. Electronic and zero-point energies, SOMO energies and NBO analysis of spin densities on phosphorus for radical cations derived from primary phosphines obtained by B3LYP/6-311+G(d,p).

\begin{tabular}{|c|c|c|c|c|}
\hline $\mathrm{N}$ & $E_{\mathrm{SCF}} /$ a.u. & $E_{\mathrm{ZPV}} /$ a.u. & SOMO energy / eV & Spin density on P \\
\hline $\mathbf{3}^{\cdot+}$ & -732.510574 & 0.248469 & -11.7 & 0.693 \\
\hline $\mathbf{3 4}^{\bullet+}$ & -807.765251 & 0.252541 & -11.3 & 0.530 \\
\hline $\mathbf{4 9}^{\bullet+}$ & -1074.492839 & 0.256247 & -10.8 & 0.379 \\
\hline $\mathbf{2 9}^{\cdot+}$ & -887.403616 & 0.320046 & -11.0 & 0.587 \\
\hline $\mathbf{3 6}^{\circ+}$ & -962.649686 & 0.323942 & -10.3 & 0.673 \\
\hline $\mathbf{3 3}^{\cdot+}$ & -1229.380979 & 0.327369 & -9.9 & 0.327 \\
\hline
\end{tabular}




\section{Stationary structures for secondary diamondoid phosphines}

The conformational dynamics of 4-diamantylphenylphosphine (38) were computed at the dispersion-corrected B3LYP-D3(BJ)/6-311+G(d,p) level of theory with Becke-Johnson (BJ) damping. ${ }^{1}$ The configurational stability of $\mathbf{3 8}$ is quite high as the energy through a planar inversion (around P) transition structure is $31.0 \mathrm{kcal} \mathrm{mol}^{-1}$. This high value agrees well with the experimental enantiomerization barriers for acyclic phosphines $\left(30-35 \mathrm{kcal} \mathrm{mol}^{-1}\right){ }^{2}$ and it makes 38 a potential target for enantioseparation. The rotation around the C4-P bond in 38a through transition structure TS1 requires a very low activation barrier of only $2.7 \mathrm{kcal}$ $\mathrm{mol}^{-1}\left(\Delta H^{\ddagger}{ }_{298}\right.$, Figure S1). The rotation of the phenyl group through TS2 is accompanied by a barrier of $4.4 \mathrm{kcal} \mathrm{mol}^{-1}$.

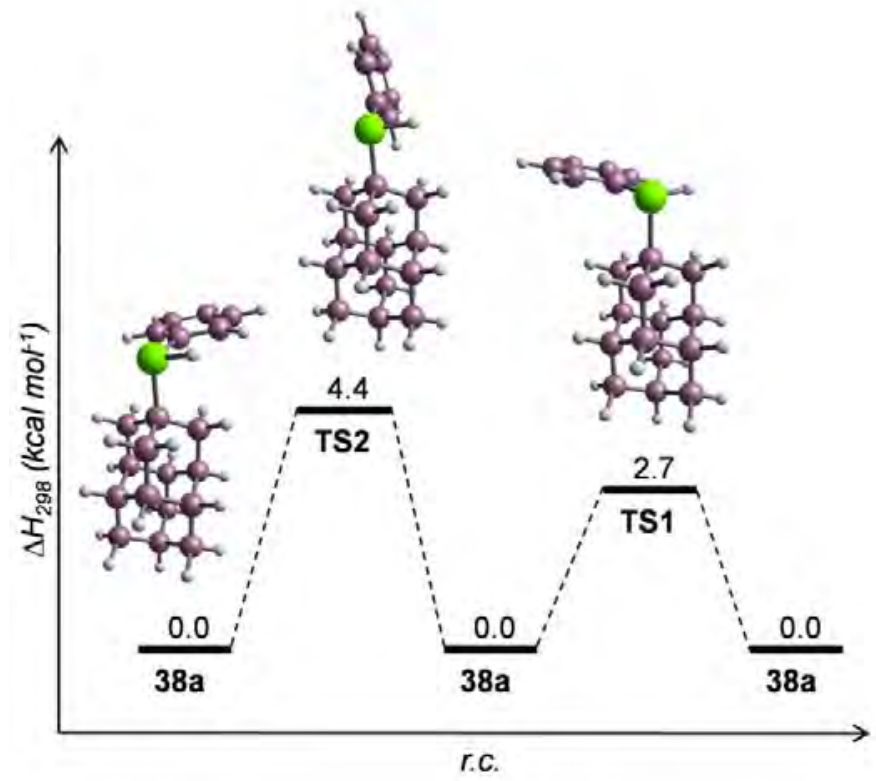

Figure S1. Stationary structures for the rotation around the C4-P bond of 38 (B3LYP$\mathrm{D} 3(\mathrm{BJ}) / 6-311+\mathrm{G}(\mathrm{d}, \mathrm{p})$ ); enthalpies in $\mathrm{kcal} \mathrm{mol}^{-1}$; r.c. stands for reaction coordinate.

Computations for 1-diamantylphenylphosphine (39) indicate that the rotation around the C1-P bond results in the formation of rotamers 39a, 39b, and 39c (Figure S2).

Sterically highly congested conformer 39c is slightly higher in energy than 39a and 39b and they are both likely to contribute to the conformational population as their interconversion through TS4 requires an energy of only $2.2 \mathrm{kcal} \mathrm{mol}^{-1}$. The full rotation through TS6 is slightly less facile $\left(\Delta H^{\ddagger}{ }_{298}=4.2 \mathrm{kcal} \mathrm{mol}^{-1}\right)$. Similarly to 38 , the rotation of the phenyl group (TS3 and TS5) is characterized by moderate barriers of 4.6 and $4.8 \mathrm{kcal} \mathrm{mol}^{-1}$ (39a and 39b, respectively). The phenyl rotation in $39 c$ is hampered due to strong repulsions with the cage hydrogens of the diamantane moiety. We located a number of transition structures for the enantiomerization, for which the least unfavorable TS8 lies very high in energy $\left(\Delta H_{298}^{\ddagger}=31.4 \mathrm{kcal} \mathrm{mol}^{-1}\right)$ indicating the configurational stability of phosphine 39 at room temperature. 


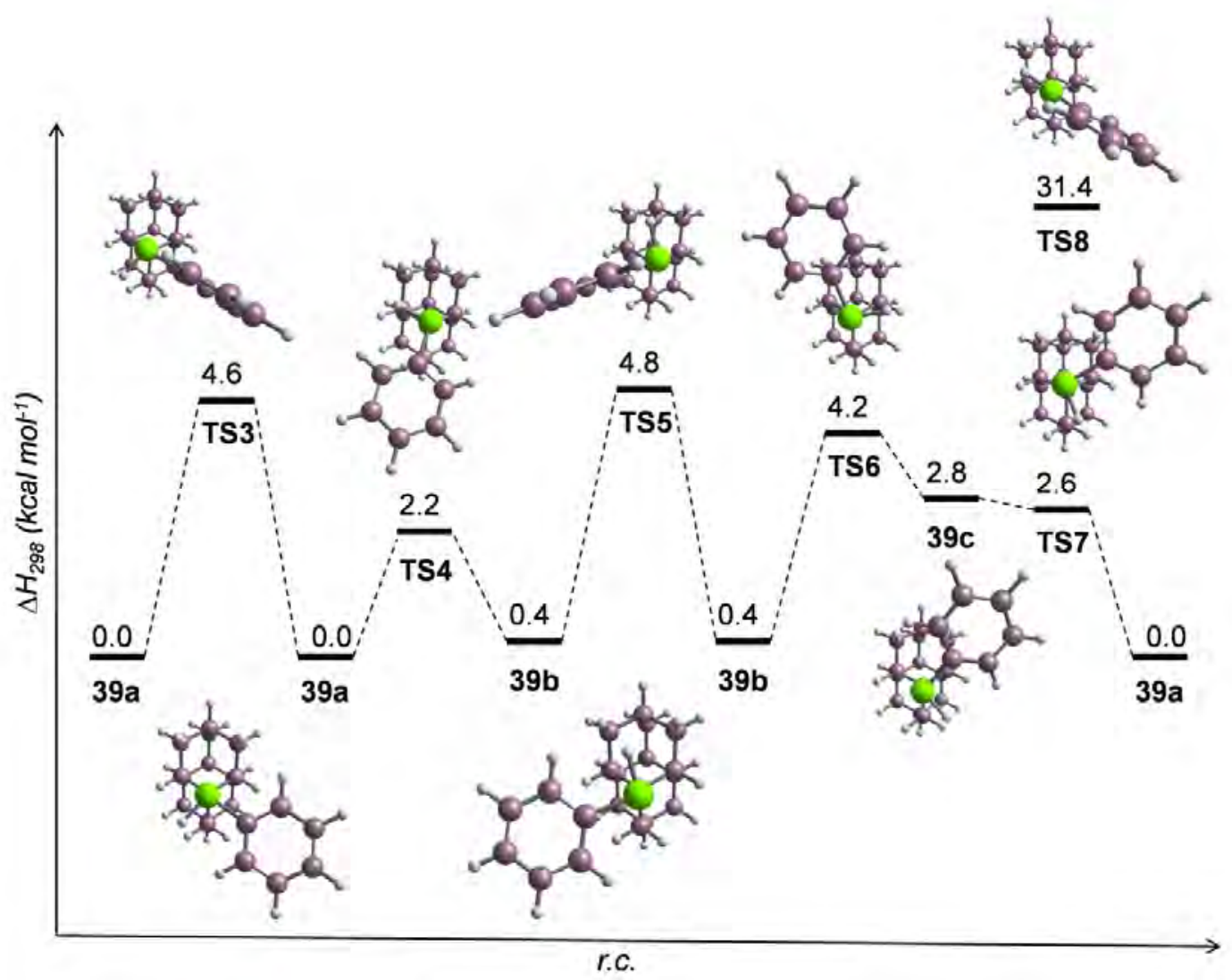

Figure S2. Stationary structures for the rotation around the C1-P bonds of phosphine $\mathbf{3 9}$ at B3LYP-D3(BJ)/6-311+G(d,p); enthalpies in $\mathrm{kcal} \mathrm{mol}^{-1}$; r.c. stands for reaction coordinate.

\section{Coordinates of the computed transition states and minima from figures S1 and S2}

- 38a

$\begin{array}{lccc}\text { C } & -1.33829 & -0.25072 & -2.34059 \\ \text { C } & 0.4307 & 1.35383 & -1.54969 \\ \text { C } & -0.66022 & 1.10669 & -2.60858 \\ \text { C } & -0.28607 & -1.3734 & -2.41552 \\ \text { C } & 1.48262 & 0.23096 & -1.62194 \\ \text { C } & 0.80349 & -1.12623 & -1.35562 \\ \text { H } & -2.1206 & -0.42594 & -3.08647 \\ \text { H } & 0.90986 & 2.32155 & -1.73247 \\ \text { H } & -0.76092 & -2.34704 & -2.24816 \\ \text { H } & 2.27359 & 0.40673 & -0.88446 \\ \text { C } & 0.16238 & -1.11357 & 0.04881 \\ \text { H } & 1.55006 & -1.92639 & -1.4003 \\ \text { C } & -0.21116 & 1.36679 & -0.14626 \\ \text { C } & -1.97791 & -0.23729 & -0.93614 \\ \text { H } & 0.55647 & 1.5683 & 0.60805 \\ \text { H } & -2.48237 & -1.19284 & -0.75056 \\ \text { H } & 0.93545 & -0.96924 & 0.80902 \\ \text { H } & -0.30575 & -2.0842 & 0.24978 \\ \text { C } & -0.89365 & 0.00819 & 0.14294 \\ \text { P } & -1.80192 & 0.09737 & 1.81093 \\ \text { C } & -0.45427 & 0.00429 & 3.07287 \\ \text { C } & 0.10096 & 1.20018 & 3.55094 \\ \text { C } & 1.09878 & 1.18944 & 4.5227 \\ \text { C } & 1.54257 & -0.01891 & 5.05587 \\ \text { C } & 0.0009 & -1.20312 & 3.62219 \\ \text { C } & 0.98563 & -1.21478 & 4.60754 \\ & & & \end{array}$




$\begin{array}{lccl}\mathrm{H} & -0.25959 & 2.14941 & 3.16934 \\ \mathrm{H} & 1.51818 & 2.1253 & 4.87395 \\ \mathrm{H} & -0.4177 & -2.14205 & 3.27896 \\ \mathrm{H} & 1.31986 & -2.15911 & 5.02254 \\ \mathrm{H} & -2.16402 & -1.27782 & 1.86172 \\ \mathrm{H} & 2.31057 & -0.02836 & 5.82041 \\ \mathrm{C} & 2.14942 & 0.22439 & -2.99738 \\ \mathrm{C} & 0.33617 & -1.41627 & -3.81104 \\ \mathrm{C} & -0.04657 & 1.11989 & -4.0085 \\ \mathrm{H} & -0.98059 & 2.16991 & -0.10511 \\ \mathrm{H} & -1.4225 & 1.91497 & -2.54271 \\ \mathrm{H} & -2.72693 & 0.58433 & -0.88498 \\ \mathrm{H} & 2.8932 & -0.57521 & -3.0378 \\ \mathrm{H} & 2.63885 & 1.18619 & -3.16951 \\ \mathrm{H} & -0.82894 & 0.94372 & -4.75084 \\ \mathrm{H} & 0.41869 & 2.09131 & -4.19314 \\ \mathrm{C} & 1.01062 & 0.02071 & -4.11179 \\ \mathrm{H} & 1.08051 & -2.21525 & -3.85359 \\ \mathrm{H} & -0.44424 & -1.60562 & -4.55219 \\ \mathrm{H} & 1.44933 & 0.03015 & -5.11262\end{array}$

- TS1

$\begin{array}{lccc}\text { C } & -1.34959 & -0.12912 & 2.34285 \\ \mathrm{C} & 0.7485 & -1.15741 & 1.4129 \\ \mathrm{C} & -0.33669 & -1.2786 & 2.49907 \\ \mathrm{C} & -0.62171 & 1.22003 & 2.49285 \\ \mathrm{C} & 1.47597 & 0.19209 & 1.56021 \\ \mathrm{C} & 0.46212 & 1.34088 & 1.40563 \\ \mathrm{H} & -2.12763 & -0.2171 & 3.10819 \\ \mathrm{H} & 1.46625 & -1.97736 & 1.51601 \\ \mathrm{H} & -1.33638 & 2.04567 & 2.40548 \\ \mathrm{H} & 2.26276 & 0.28107 & 0.80312 \\ \mathrm{C} & -0.19519 & 1.26185 & 0.01061 \\ \mathrm{H} & 0.97742 & 2.30225 & 1.50389 \\ \mathrm{C} & 0.09048 & -1.23699 & 0.01883 \\ \mathrm{C} & -2.00529 & -0.20857 & 0.94773 \\ \mathrm{H} & 0.86096 & -1.17762 & -0.75676 \\ \mathrm{H} & -2.74445 & 0.59331 & 0.84112 \\ \mathrm{H} & 0.56553 & 1.37808 & -0.76656 \\ \mathrm{H} & -0.90361 & 2.08877 & -0.11059 \\ \mathrm{C} & -0.92758 & -0.08604 & -0.15841 \\ \mathrm{P} & -1.83088 & -0.28952 & -1.81943 \\ \mathrm{C} & -0.45802 & -0.00511 & -3.0391 \\ \mathrm{C} & 0.39608 & -1.10162 & -3.22442 \\ \mathrm{C} & 1.43029 & -1.05613 & -4.15626 \\ \mathrm{C} & 1.61383 & 0.07937 & -4.94169 \\ \mathrm{C} & -0.26183 & 1.12876 & -3.83977 \\ \mathrm{C} & 0.76031 & 1.16901 & -4.78532 \\ \mathrm{H} & 0.24301 & -2.00462 & -2.64388 \\ \mathrm{H} & 2.08229 & -1.91297 & -4.27922 \\ \mathrm{H} & -0.91328 & 1.98702 & -3.72526 \\ \mathrm{H} & 0.89152 & 2.05368 & -5.39773 \\ \mathrm{H} & -2.53694 & 0.94374 & -1.76089 \\ \mathrm{H} & 2.41072 & 0.11187 & -5.67529 \\ \mathrm{C} & 2.15885 & 0.27399 & 2.92561 \\ \mathrm{C} & 0.0094 & 1.32384 & 3.8815 \\ \mathrm{C} & 0.30069 & -1.23125 & 3.88792 \\ \mathrm{H} & -0.44595 & -2.20737 & -0.07585 \\ & & & \\ & & & \end{array}$




$\begin{array}{cccl}\mathrm{H} & -0.86572 & -2.25033 & 2.37925 \\ \mathrm{H} & -2.51762 & -1.19076 & 0.8414 \\ \mathrm{H} & 2.67143 & 1.23439 & 3.01958 \\ \mathrm{H} & 2.88465 & -0.53686 & 3.01847 \\ \mathrm{H} & -0.47779 & -1.31801 & 4.6498 \\ \mathrm{H} & 1.00606 & -2.05852 & 3.99278 \\ \mathrm{C} & 1.0403 & 0.09458 & 4.06427 \\ \mathrm{H} & 0.52277 & 2.28361 & 3.97751 \\ \mathrm{H} & -0.77065 & 1.2504 & 4.64318 \\ \mathrm{H} & 1.49599 & 0.12839 & 5.05722\end{array}$

- TS2

\begin{tabular}{|c|c|c|c|}
\hline C & -1.40713 & $-\odot .04427$ & -2.3651 \\
\hline C & 0.58379 & 1.26742 & -1.56649 \\
\hline C & $-\odot .58032$ & 1.23772 & -2.5742 \\
\hline C & $-\odot .50794$ & -1.27544 & -2.58288 \\
\hline C & 1.48277 & ๑. 03583 & -1.78153 \\
\hline C & 0.6548 & -1.24558 & -1.57399 \\
\hline $\mathrm{H}$ & -2.24097 & $-\odot .06451$ & -3.07424 \\
\hline $\mathrm{H}$ & 1.16941 & 2.18116 & -1.70699 \\
\hline $\mathrm{H}$ & -1.09083 & -2.19431 & -2.45837 \\
\hline H & 2.3248 & $\odot .0564$ & -1.08123 \\
\hline C & ๑. 09129 & -1.2664 & -0.13666 \\
\hline $\mathrm{H}$ & 1.29282 & -2.12341 & -1.72032 \\
\hline C & $\odot .0194$ & 1.24708 & -0.13005 \\
\hline C & -1.96913 & -0.0644 & -0.92779 \\
\hline $\mathrm{H}$ & $\odot .84242$ & 1.29453 & 0.59024 \\
\hline $\mathrm{H}$ & -2.5782 & $-\odot .9633$ & -0.78208 \\
\hline H & $\odot .91286$ & -1.27657 & 0.58505 \\
\hline $\mathrm{H}$ & $-\odot .48289$ & -2.18577 & ๑. 02116 \\
\hline C & $-\odot .81086$ & $-\odot .03716$ & $\odot .10036$ \\
\hline $\mathrm{P}$ & -1.61642 & $\odot . \odot 292$ & 1.82148 \\
\hline C & $-\odot .40947$ & $-\odot .01346$ & 3.25899 \\
\hline C & 0.96087 & $-\odot .05953$ & 2.96629 \\
\hline C & 1.91213 & $-\odot . \odot 6 \odot 3$ & 3.9834 \\
\hline C & 1.51117 & $\odot .01241$ & 5.31517 \\
\hline C & -0.79623 & $\odot .06271$ & 4.60415 \\
\hline C & $\odot .15434$ & 0.08079 & 5.62204 \\
\hline H & 1.28789 & $-\odot . \odot 8171$ & 1.9328 \\
\hline $\mathrm{H}$ & 2.96602 & $-\odot .10218$ & 3.73493 \\
\hline $\mathrm{H}$ & -1.84813 & ๑.10969 & 4.8592 \\
\hline $\mathrm{H}$ & -0.16596 & 0.14526 & 6.65541 \\
\hline $\mathrm{H}$ & -2.14329 & -1.29125 & 1.79428 \\
\hline $\mathrm{H}$ & 2.25049 & $\odot .02367$ & 6.10718 \\
\hline C & 2.07401 & 0.05729 & -3.19111 \\
\hline C & 0.03364 & -1.28289 & -4.01241 \\
\hline C & $-\odot .04 \odot 57$ & 1.28699 & -4.00359 \\
\hline $\mathrm{H}$ & -0.641 & 2.13082 & 0.01429 \\
\hline H & -1.23186 & 2.12376 & -2.40529 \\
\hline $\mathrm{H}$ & -2.60657 & 0.83442 & -0.77333 \\
\hline $\mathrm{H}$ & 2.70949 & $-\odot .81998$ & -3.33297 \\
\hline H & 2.6696 & 0.96314 & -3.3220 \\
\hline $\mathrm{H}$ & $-\odot .87438$ & 1.26545 & -4.70925 \\
\hline $\mathrm{H}$ & 0.53173 & 2.20624 & -4.14493 \\
\hline C & ๑.86511 & ๑.08069 & -4.24812 \\
\hline $\mathrm{H}$ & 0.66965 & -2.15944 & -4.15635 \\
\hline $\mathrm{H}$ & -0.79985 & -1.31787 & -4.71792 \\
\hline $\mathrm{H}$ & 1.25098 & 0.11596 & -5.27004 \\
\hline
\end{tabular}




\begin{tabular}{|c|c|c|c|}
\hline C & ๑. 25524 & 0.87185 & 2.81937 \\
\hline C & $\odot .85587$ & -1.33009 & 1.79288 \\
\hline C & $\odot .25497$ & $-\odot .65621$ & 3.04535 \\
\hline C & 1.68784 & 1.37048 & 2.59916 \\
\hline C & 2.29049 & -0.84038 & 1.5654 \\
\hline C & 2.28349 & 0.68107 & 1.36403 \\
\hline C & -1.16884 & -1.1656 & 3.2995 \\
\hline $\mathrm{H}$ & -0.17329 & 1.36308 & 3.70101 \\
\hline $\mathrm{H}$ & ๑.85748 & -2.41685 & 1.938 \\
\hline $\mathrm{H}$ & 1.69114 & 2.45755 & 2.45986 \\
\hline $\mathrm{H}$ & 2.72092 & -1.33255 & ๑. 6862 \\
\hline C & 1.4174 & 1.01462 & $\odot .14228$ \\
\hline $\mathrm{H}$ & 3.30422 & 1.03864 & 1.1925 \\
\hline C & $-\odot .04402$ & -1.00743 & $\odot .57317$ \\
\hline C & -0.64739 & 1.19484 & 1.60022 \\
\hline $\mathrm{H}$ & 0.37398 & -1.50635 & -0.30684 \\
\hline $\mathrm{H}$ & -0.66717 & 2.28218 & 1.45596 \\
\hline $\mathrm{H}$ & 1.84438 & $\odot .55209$ & -0.75144 \\
\hline $\mathrm{H}$ & 1.41809 & 2.09734 & $-\odot .02573$ \\
\hline C & -0.03054 & $\odot .52771$ & ๑. 33691 \\
\hline$P$ & -1.09624 & $\odot .99 \odot \odot 2$ & -1.18115 \\
\hline C & -2.07254 & ๑.68383 & 1.87375 \\
\hline C & -1.46784 & -1.52488 & 0.83916 \\
\hline C & -0.37558 & 0.36577 & -2.5146 \\
\hline C & 0.71677 & -0.45043 & -2.42058 \\
\hline C & 1.32279 & -0.96441 & -3.56363 \\
\hline C & 0.74263 & -0.76021 & -4.81415 \\
\hline C & -1.04024 & 0.49106 & -3.76983 \\
\hline C & -0.44495 & -0.03799 & -4.91255 \\
\hline $\mathrm{H}$ & 1.16281 & -0.63829 & -1.4501 \\
\hline $\mathrm{H}$ & 2.24111 & -1.53419 & -3.47654 \\
\hline $\mathrm{H}$ & -1.95701 & 1.062 & -3.86101 \\
\hline $\mathrm{H}$ & -0.90642 & 0.11893 & -5.88115 \\
\hline $\mathrm{H}$ & -0.54863 & 2.29362 & -1.33357 \\
\hline $\mathrm{H}$ & 1.20859 & -1.16711 & -5.70393 \\
\hline $\mathrm{H}$ & 2.91589 & -1.10817 & 2.42457 \\
\hline $\mathrm{H}$ & 2.29614 & 1.15761 & 3.48552 \\
\hline $\mathrm{H}$ & ๑. 88821 & -0.88598 & 3.91075 \\
\hline $\mathrm{H}$ & -1.57929 & -0.69793 & 4.2015 \\
\hline C & -2.05142 & -0.83914 & 2.08596 \\
\hline $\mathrm{H}$ & -1.15321 & -2.24712 & 3.47654 \\
\hline $\mathrm{H}$ & -2.73637 & 0.93948 & 1.04458 \\
\hline $\mathrm{H}$ & -2.46764 & 1.17907 & 2.76759 \\
\hline $\mathrm{H}$ & -2.11452 & -1.33975 & $-\odot .02301$ \\
\hline $\mathrm{H}$ & -1.43629 & -2.60981 & $\odot .98937$ \\
\hline $\mathrm{H}$ & -3.07054 & -1.19879 & 2.25945 \\
\hline
\end{tabular}

- $\quad$ TS3

$\begin{array}{rr}\text { C } & 0.25524 \\ \text { C } & 0.85587 \\ \text { C } & 0.25497 \\ \text { C } & 1.68784 \\ \text { C } & 2.29049 \\ \text { C } & 2.28349 \\ \text { C } & -1.16884 \\ \text { H } & -0.17329\end{array}$

$$
0.87185
$$$$
\text { 2. } 81937
$$$$
\text { 1. } 79288
$$$$
\text { 3. } 04535
$$$$
\text { 2. } 59916
$$$$
\text { 1. } 5654
$$$$
\text { 1. } 36403
$$$$
\text { 3. } 2995
$$$$
\text { 3. } 70101
$$ 


\begin{tabular}{|c|c|c|c|}
\hline $\mathrm{H}$ & ๑. 85748 & -2.41685 & 1.938 \\
\hline $\mathrm{H}$ & 1.69114 & 2.45755 & 2.45986 \\
\hline $\mathrm{H}$ & 2.72092 & -1.33255 & 0.6862 \\
\hline C & 1.4174 & 1.01462 & $\odot .14228$ \\
\hline H & 3.30422 & 1.03864 & 1.1925 \\
\hline C & $-\odot .044 \odot 2$ & -1.00743 & 0.57317 \\
\hline C & -0.64739 & 1.19484 & 1.60022 \\
\hline $\mathrm{H}$ & ๑. 37398 & -1.50635 & -0.30684 \\
\hline $\mathrm{H}$ & $-\odot .66717$ & 2.28218 & 1.45596 \\
\hline $\mathrm{H}$ & 1.84438 & $\odot .55209$ & -0.75144 \\
\hline $\mathrm{H}$ & 1.41809 & 2.09734 & -0.02573 \\
\hline C & $-\odot .03054$ & 0.52771 & 0.33691 \\
\hline $\mathrm{P}$ & -1.09624 & $\odot .990 \odot 2$ & -1.18115 \\
\hline C & -2.07254 & $\odot .68383$ & 1.87375 \\
\hline C & -1.46784 & -1.52488 & ๑.83916 \\
\hline C & $-\odot .37558$ & 0.36577 & -2.5146 \\
\hline C & 0.71677 & -0.45043 & -2.42058 \\
\hline C & 1.32279 & -0.96441 & -3.56363 \\
\hline C & $\odot .74263$ & -0.76021 & -4.81415 \\
\hline C & -1.04024 & ๑. 49106 & -3.76983 \\
\hline C & $-\odot .44495$ & -0.03799 & -4.91255 \\
\hline $\mathrm{H}$ & 1.16281 & -0.63829 & -1.4501 \\
\hline $\mathrm{H}$ & 2.24111 & -1.53419 & -3.47654 \\
\hline H & -1.95701 & 1.062 & -3.86101 \\
\hline $\mathrm{H}$ & $-\odot .90642$ & ๑.11893 & -5.88115 \\
\hline H & $-\odot .54863$ & 2.29362 & -1.33357 \\
\hline H & 1.20859 & -1.16711 & -5.70393 \\
\hline $\mathrm{H}$ & 2.91589 & -1.10817 & 2.42457 \\
\hline $\mathrm{H}$ & 2.29614 & 1.15761 & 3.48552 \\
\hline $\mathrm{H}$ & ๑.88821 & $-\odot .88598$ & 3.91075 \\
\hline H & -1.57929 & -0.69793 & 4.2015 \\
\hline C & -2.05142 & -0.83914 & 2.08596 \\
\hline H & -1.15321 & -2.24712 & 3.47654 \\
\hline H & -2.73637 & 0.93948 & 1.04458 \\
\hline $\mathrm{H}$ & -2.46764 & 1.17907 & 2.76759 \\
\hline $\mathrm{H}$ & -2.11452 & -1.33975 & -0.02301 \\
\hline $\mathrm{H}$ & -1.43629 & -2.60981 & 0.98937 \\
\hline H & -3.07054 & -1.19879 & 2.25945 \\
\hline
\end{tabular}

- TS4

$\begin{array}{lccl}\text { C } & 0.73223 & 1.27458 & 2.36295 \\ \text { C } & 0.79051 & -1.23992 & 2.4022 \\ \text { C } & 0.59977 & 0.0268 & 3.25705 \\ \text { C } & 2.13041 & 1.29688 & 1.71677 \\ \text { C } & 2.1874 & -1.2181 & 1.7537 \\ \text { C } & 2.31973 & 0.03082 & 0.86122 \\ \text { C } & -0.77198 & 0.00511 & 3.92944 \\ \text { H } & 0.58858 & 2.17719 & 2.96585 \\ \text { H } & 0.68941 & -2.12935 & 3.03311 \\ \text { H } & 2.24455 & 2.19242 & 1.09511 \\ \text { H } & 2.34067 & -2.12421 & 1.15731 \\ \text { C } & 1.2422 & -0.0104 & -0.24344 \\ \text { H } & 3.31001 & 0.04601 & 0.39369 \\ \text { C } & -0.28725 & -1.28037 & 1.29862 \\ \text { C } & -0.34462 & 1.23184 & 1.25857 \\ \text { H } & -0.17207 & -2.19261 & 0.70436 \\ \text { H } & -0.26954 & 2.12996 & 0.63435 \\ \text { H } & 1.38976 & -0.89158 & -0.87409\end{array}$




$\begin{array}{lccc}\text { H } & 1.34488 & 0.86717 & -0.89215 \\ \mathrm{C} & -0.16656 & -0.03598 & 0.38664 \\ \mathrm{P} & -1.41881 & 0.01191 & -1.06451 \\ \mathrm{C} & -1.73644 & 1.24246 & 1.88876 \\ \mathrm{C} & -1.67713 & -1.32924 & 1.93128 \\ \mathrm{C} & -0.58798 & 0.02931 & -2.71564 \\ \mathrm{C} & -0.30971 & -1.1956 & -3.3394 \\ \mathrm{C} & 0.2888 & -1.24045 & -4.59621 \\ \mathrm{C} & 0.59659 & -0.05836 & -5.26672 \\ \mathrm{C} & -0.27518 & 1.20986 & -3.40524 \\ \mathrm{C} & 0.30644 & 1.16691 & -4.67026 \\ \mathrm{H} & -0.57557 & -2.12308 & -2.84347 \\ \mathrm{H} & 0.50033 & -2.19827 & -5.05767 \\ \mathrm{H} & -0.48855 & 2.17076 & -2.95161 \\ \mathrm{H} & 0.53577 & 2.0915 & -5.18833 \\ \mathrm{H} & -1.68782 & 1.40805 & -1.00616 \\ \mathrm{H} & 1.05157 & -0.09158 & -6.24965 \\ \mathrm{H} & 2.96177 & -1.21116 & 2.52891 \\ \mathrm{H} & 2.90221 & 1.34747 & 2.49297 \\ \mathrm{H} & 1.34662 & 0.0569 & 4.05814 \\ \mathrm{H} & -0.89625 & 0.90715 & 4.53238 \\ \mathrm{C} & -1.78988 & -0.04442 & 2.79457 \\ \mathrm{H} & -0.8491 & -0.87531 & 4.57153 \\ \mathrm{H} & -2.49268 & 1.21207 & 1.10124 \\ \mathrm{H} & -1.86194 & 2.15266 & 2.47892 \\ \mathrm{H} & -2.43428 & -1.35768 & 1.14457 \\ \mathrm{H} & -1.76341 & -2.22364 & 2.55254 \\ \mathrm{H} & -2.77594 & -0.06023 & 3.22509\end{array}$

- $39 b$

$\begin{array}{lccc}\text { C } & -0.83529 & -1.34505 & 1.83572 \\ \text { C } & -0.16788 & 0.81158 & 2.91615 \\ \text { C } & -0.17012 & -0.72345 & 3.08336 \\ \text { C } & -2.27484 & -0.83774 & 1.69938 \\ \text { C } & -1.60417 & 1.32899 & 2.78524 \\ \text { C } & -2.26484 & 0.69027 & 1.55692 \\ \text { C } & 1.261 & -1.24647 & 3.25182 \\ \text { H } & -0.8406 & -2.4363 & 1.94099 \\ \text { H } & 0.30786 & 1.26689 & 3.79269 \\ \text { H } & -2.7524 & -1.29266 & 0.82457 \\ \text { H } & -1.60364 & 2.42004 & 2.68516 \\ \text { C } & -1.45961 & 1.06147 & 0.30691 \\ \text { H } & -3.28916 & 1.06107 & 1.44933 \\ \text { C } & 0.674 & 1.17487 & 1.6662 \\ \text { C } & 0.00938 & -0.98444 & 0.5844 \\ \text { H } & 0.69507 & 2.26512 & 1.55756 \\ \text { H } & -0.44998 & -1.4473 & -0.29385 \\ \text { H } & -1.46009 & 2.14893 & 0.17137 \\ \text { H } & -1.93711 & 0.62738 & -0.57581 \\ \text { C } & -0.00269 & 0.5572 & 0.4099 \\ \text { P } & 0.8573 & 1.23142 & -1.1511 \\ \text { C } & 1.43352 & -1.52935 & 0.77439 \\ \text { C } & 2.10647 & 0.64608 & 1.84899 \\ \text { C } & 0.369 & 0.43154 & -2.69274 \\ \text { C } & -0.61568 & -0.55462 & -2.55073 \\ \text { C } & -1.10815 & -1.23954 & -3.65872 \\ \text { C } & -0.6389 & -0.93388 & -4.9342 \\ \text { C } & 0.82573 & 0.73343 & -3.98299\end{array}$




$\begin{array}{lrcl}\mathrm{C} & 0.32443 & 0.05982 & -5.09379 \\ \mathrm{H} & -1.00284 & -0.78322 & -1.56405 \\ \mathrm{H} & -1.86525 & -2.0037 & -3.52725 \\ \mathrm{H} & 1.58067 & 1.49778 & -4.12067 \\ \mathrm{H} & 0.68895 & 0.30904 & -6.08387 \\ \mathrm{H} & 2.05417 & 0.47554 & -1.11867 \\ \mathrm{H} & -1.02591 & -1.46071 & -5.79847 \\ \mathrm{H} & -2.17134 & 1.08825 & 3.69129 \\ \mathrm{H} & -2.85929 & -1.13465 & 2.5776 \\ \mathrm{H} & -0.76346 & -0.98338 & 3.96822 \\ \mathrm{H} & 1.24912 & -2.33303 & 3.39271 \\ \mathrm{C} & 2.08551 & -0.88421 & 2.00815 \\ \mathrm{H} & 1.71605 & -0.81092 & 4.14844 \\ \mathrm{H} & 2.04299 & -1.34562 & -0.1126 \\ \mathrm{H} & 1.38789 & -2.61609 & 0.90491 \\ \mathrm{H} & 2.73881 & 0.93469 & 1.00492 \\ \mathrm{H} & 2.54866 & 1.10529 & 2.7398 \\ \mathrm{H} & 3.10913 & -1.25526 & 2.11965\end{array}$

- $\quad$ TS5

$\begin{array}{lccc}\text { C } & -0.87123 & -1.33009 & 1.78944 \\ \mathrm{C} & -0.20626 & 0.82174 & 2.88189 \\ \mathrm{C} & -0.23887 & -0.71234 & 3.05642 \\ \mathrm{C} & -2.29721 & -0.8008 & 1.60118 \\ \mathrm{C} & -1.62897 & 1.36108 & 2.69917 \\ \mathrm{C} & -2.25781 & 0.72624 & 1.45171 \\ \mathrm{C} & 1.17733 & -1.25707 & 3.27656 \\ \mathrm{H} & -0.89767 & -2.42066 & 1.89978 \\ \mathrm{H} & 0.24677 & 1.27419 & 3.77222 \\ \mathrm{H} & -2.75204 & -1.25283 & 0.71251 \\ \mathrm{H} & -1.6075 & 2.45159 & 2.59387 \\ \mathrm{C} & -1.40455 & 1.07802 & 0.22803 \\ \mathrm{H} & -3.27197 & 1.11266 & 1.30716 \\ \mathrm{C} & 0.68354 & 1.16502 & 1.65962 \\ \mathrm{C} & 0.02134 & -0.98957 & 0.56587 \\ \mathrm{H} & 0.72583 & 2.25433 & 1.54645 \\ \mathrm{H} & -0.41534 & -1.44983 & -0.32562 \\ \mathrm{H} & -1.38289 & 2.16476 & 0.08724 \\ \mathrm{H} & -1.85872 & 0.64676 & -0.66863 \\ \mathrm{C} & 0.04 & 0.55132 & 0.38354 \\ \mathrm{P} & 0.96348 & 1.20356 & -1.15058 \\ \mathrm{C} & 1.42947 & -1.55593 & 0.80737 \\ \mathrm{C} & 2.1005 & 0.61457 & 1.8941 \\ \mathrm{C} & 0.29933 & 0.36545 & -2.56327 \\ \mathrm{C} & 0.91947 & 0.63611 & -3.79055 \\ \mathrm{C} & 0.46612 & 0.04534 & -4.96779 \\ \mathrm{C} & -0.60441 & -0.84588 & -4.93492 \\ \mathrm{C} & -0.7716 & -0.53911 & -2.54501 \\ \mathrm{C} & -1.21739 & -1.14204 & -3.71873 \\ \mathrm{H} & 1.76761 & 1.31121 & -3.82199 \\ \mathrm{H} & 0.95528 & 0.27265 & -5.90824 \\ \mathrm{H} & -1.26278 & -0.77187 & -1.60769 \\ \mathrm{H} & -2.04602 & -1.84066 & -3.68368 \\ \mathrm{H} & 2.14645 & 0.42898 & -1.07337 \\ \mathrm{H} & -0.95419 & -1.31214 & -5.84884 \\ \mathrm{H} & -2.23055 & 1.13417 & 3.58653 \\ \mathrm{H} & -2.91599 & -1.08378 & 2.46041 \\ \mathrm{H} & -0.86616 & -0.95818 & 3.92178\end{array}$




$\begin{array}{llll}\mathrm{H} & 1.14322 & -2.34266 & 3.4222 \\ \mathrm{C} & 2.04951 & -0.91448 & 2.05993 \\ \mathrm{H} & 1.60866 & -0.82389 & 4.1863 \\ \mathrm{H} & 2.07174 & -1.38658 & -0.05924 \\ \mathrm{H} & 1.36197 & -2.64123 & 0.94156 \\ \mathrm{H} & 2.76586 & 0.88867 & 1.0707 \\ \mathrm{H} & 2.51953 & 1.07155 & 2.79754 \\ \mathrm{H} & 3.06282 & -1.3011 & 2.20837\end{array}$

- TS6

\begin{tabular}{|c|c|c|c|}
\hline C & -0.41429 & -1.35875 & -2.3078 \\
\hline C & -0.61941 & 1.12904 & -2.47477 \\
\hline C & -1.38306 & $-\odot .20278$ & -2.63909 \\
\hline C & 0.78629 & -1.32799 & -3.26016 \\
\hline C & 0.58205 & 1.17126 & -3.42569 \\
\hline C & 1.52758 & $\odot .00675$ & -3.10385 \\
\hline C & -2.60617 & $-\odot .23966$ & -1.71502 \\
\hline $\mathrm{H}$ & $-\odot .9458$ & -2.31152 & -2.41686 \\
\hline $\mathrm{H}$ & -1.29755 & 1.95941 & -2.70266 \\
\hline $\mathrm{H}$ & 1.46351 & -2.16044 & -3.03956 \\
\hline $\mathrm{H}$ & 1.1125 & 2.12413 & -3.31975 \\
\hline C & 2.00512 & 0.1437 & -1.65243 \\
\hline $\mathrm{H}$ & 2. 39354 & 0.03415 & -3.77355 \\
\hline C & $-\odot .16773$ & 1.26605 & -0.99891 \\
\hline C & 0.03573 & -1.2229 & $-0.830 \odot 5$ \\
\hline $\mathrm{H}$ & $\odot .35268$ & 2.22201 & -0.88345 \\
\hline $\mathrm{H}$ & $\odot .70352$ & -2.05845 & -0.58807 \\
\hline $\mathrm{H}$ & 2.55891 & 1.07834 & -1.53141 \\
\hline H & 2.69686 & $-\odot .67133$ & -1.41351 \\
\hline C & ๑.81866 & 0.11248 & -0.67137 \\
\hline P & 1.71424 & ๑. 29136 & 0.92307 \\
\hline C & -1.2013 & -1.26849 & 0.08352 \\
\hline C & -1.40277 & 1.22993 & -0.08288 \\
\hline C & $\odot .73268$ & 0.13793 & 2.43947 \\
\hline C & $\odot .83562$ & -1.05159 & 3.17389 \\
\hline C & $\odot .15263$ & -1.21148 & 4.37625 \\
\hline C & $-\odot .62732$ & -0.17284 & 4.8805 \\
\hline C & $-\odot . \odot 5 \odot 87$ & 1.17558 & 2.96157 \\
\hline C & $-\odot .72 \odot 2$ & 1.02387 & 4.1735 \\
\hline H & 1.46088 & -1.85666 & 2.80457 \\
\hline H & ๑. 23919 & -2.14217 & 4.92501 \\
\hline H & -0.14135 & 2.10652 & 2.41476 \\
\hline $\mathrm{H}$ & -1.31923 & 1.83932 & 4.56287 \\
\hline H & 1.48216 & 1.68434 & 0.756 \\
\hline H & -1.15264 & $-\odot .29259$ & 5.82073 \\
\hline H & 0.23729 & 1.10643 & -4.46373 \\
\hline H & 0.4445 & -1.45622 & -4.2933 \\
\hline H & -1.70691 & $-\odot .30055$ & -3.6821 \\
\hline $\mathrm{H}$ & -3.15334 & -1.17897 & -1.85343 \\
\hline C & -2.14677 & -0.10482 & -0.25619 \\
\hline H & -3.294 & $\odot .57359$ & -1.97212 \\
\hline H & $-\odot .90342$ & -1.21765 & 1.13319 \\
\hline $\mathrm{H}$ & -1.71991 & -2.223 & -0.05889 \\
\hline H & -1.11249 & 1.35999 & ๑.96321 \\
\hline $\mathrm{H}$ & -2.06654 & 2.06321 & -0.33749 \\
\hline $\mathrm{H}$ & -3.01421 & -0.13111 & 0.41087 \\
\hline
\end{tabular}




\begin{tabular}{|c|c|c|c|}
\hline C & -0.41429 & -1.35875 & -2.3078 \\
\hline C & -0.61941 & 1.12904 & -2.47477 \\
\hline C & -1.38306 & $-\odot .20278$ & -2.63909 \\
\hline C & 0.78629 & -1.32799 & -3.26016 \\
\hline C & 0.58205 & 1.17126 & -3.42569 \\
\hline C & 1.52758 & 0.00675 & -3.10385 \\
\hline C & -2.60617 & -0.23966 & -1.71502 \\
\hline $\mathrm{H}$ & $-\odot .9458$ & -2.31152 & -2.41686 \\
\hline $\mathrm{H}$ & -1.29755 & 1.95941 & -2.70266 \\
\hline $\mathrm{H}$ & 1.46351 & -2.16044 & -3.03956 \\
\hline $\mathrm{H}$ & 1.1125 & 2.12413 & -3.31975 \\
\hline C & 2.00512 & 0.1437 & -1.65243 \\
\hline $\mathrm{H}$ & 2.39354 & 0.03415 & -3.77355 \\
\hline C & -0.16773 & 1.26605 & -0.99891 \\
\hline C & 0.03573 & -1.2229 & -0.83005 \\
\hline $\mathrm{H}$ & $\odot .35268$ & 2.22201 & -0.88345 \\
\hline $\mathrm{H}$ & $\odot .70352$ & -2.05845 & -0.58807 \\
\hline $\mathrm{H}$ & 2.55891 & 1.07834 & -1.53141 \\
\hline $\mathrm{H}$ & 2.69686 & -0.67133 & -1.41351 \\
\hline C & ๑. 81866 & 0.11248 & -0.67137 \\
\hline$P$ & 1.71424 & ๑. 29136 & $\odot .92307$ \\
\hline C & -1.2013 & -1.26849 & ๑. . 08352 \\
\hline C & -1.40277 & 1.22993 & -0.08288 \\
\hline C & 0.73268 & $\odot .13793$ & 2.43947 \\
\hline C & ๑. 83562 & -1.05159 & 3.17389 \\
\hline C & 0.15263 & -1.21148 & 4.37625 \\
\hline C & -0.62732 & -0.17284 & 4.8805 \\
\hline C & -0.05087 & 1.17558 & 2.96157 \\
\hline C & $-\odot .7202$ & 1.02387 & 4.1735 \\
\hline $\mathrm{H}$ & 1.46088 & -1.85666 & 2.80457 \\
\hline $\mathrm{H}$ & ๑. 23919 & -2.14217 & 4.92501 \\
\hline$H$ & -0.14135 & 2.10652 & 2.41476 \\
\hline $\mathrm{H}$ & -1.31923 & 1.83932 & 4.56287 \\
\hline $\mathrm{H}$ & 1.48216 & 1.68434 & 0.756 \\
\hline $\mathrm{H}$ & -1.15264 & $-\odot .29259$ & 5.82073 \\
\hline $\mathrm{H}$ & ๑. 23729 & 1.10643 & -4.46373 \\
\hline $\mathrm{H}$ & $\odot .4445$ & -1.45622 & -4.2933 \\
\hline $\mathrm{H}$ & -1.70691 & -0.30055 & -3.6821 \\
\hline $\mathrm{H}$ & -3.15334 & -1.17897 & -1.85343 \\
\hline C & -2.14677 & -0.10482 & -0.25619 \\
\hline $\mathrm{H}$ & -3.294 & 0.57359 & -1.97212 \\
\hline $\mathrm{H}$ & -0.90342 & -1.21765 & 1.13319 \\
\hline $\mathrm{H}$ & -1.71991 & -2.223 & -0.05889 \\
\hline $\mathrm{H}$ & -1.11249 & 1.35999 & 0.96321 \\
\hline $\mathrm{H}$ & -2.06654 & 2.06321 & -0.33749 \\
\hline $\mathrm{H}$ & -3.01421 & -0.13111 & ๑. 41087 \\
\hline
\end{tabular}

- $\quad$ TS7

$\begin{array}{lr}\text { C } & -0.49176 \\ \text { C } & -0.33553 \\ \text { C } & -1.23888 \\ \text { C } & 0.79562 \\ \text { C } & 0.95307 \\ \text { C } & 1.69874 \\ \text { C } & -2.53436 \\ \text { H } & -1.13438 \\ \text { H } & -0.8662\end{array}$

0.64661
-1.58879
-0.66648
0.33408
-1.90048
-0.58695
-0.37142
1.30846
-2.52023

2.8363

1. 69141

2. 53169

3. 62233

2. 47525

2. 78094

1. 77751

3. 42634

1. 46646 


\begin{tabular}{|c|c|c|c|}
\hline $\mathrm{H}$ & 1.325 & 1.26312 & 3.86416 \\
\hline $\mathrm{H}$ & 1.59554 & -2.57111 & 1.89364 \\
\hline C & 2.06212 & 0.11779 & 1.45649 \\
\hline $\mathrm{H}$ & 2.6189 & -0.80572 & 3.33308 \\
\hline C & ๑.02674 & -0.88368 & 0.36749 \\
\hline C & -0.1281 & 1.3495 & 1.51163 \\
\hline $\mathrm{H}$ & ๑. 64751 & -1.54593 & -0.245 \\
\hline $\mathrm{H}$ & ๑. 38253 & 2.29635 & 1.72394 \\
\hline $\mathrm{H}$ & 2.72889 & -0.5186 & $\odot .8673$ \\
\hline $\mathrm{H}$ & 2.60953 & 1.04364 & 1.6691 \\
\hline C & ๑.78301 & $\odot .43608$ & 0.65358 \\
\hline$P$ & 1.28124 & 1.29669 & -0.91318 \\
\hline C & -1.4006 & 1.69315 & 0.73907 \\
\hline C & -1.24312 & $-\odot .59361$ & -0.43094 \\
\hline C & 0.59312 & $\odot .40693$ & -2.38029 \\
\hline C & -0.75181 & $\odot .61338$ & -2.71991 \\
\hline C & -1.31428 & $-\odot .0 \odot 913$ & -3.83185 \\
\hline C & -0.53383 & -0.83048 & -4.64292 \\
\hline C & 1.36586 & -0.41988 & -3.20884 \\
\hline C & ๑.8०972 & -1.02843 & -4.33188 \\
\hline $\mathrm{H}$ & -1.36171 & 1.27756 & -2.11678 \\
\hline $\mathrm{H}$ & -2.35757 & 0.15989 & -4.07288 \\
\hline $\mathrm{H}$ & 2.41076 & $-\odot .58904$ & -2.97622 \\
\hline $\mathrm{H}$ & 1.42608 & -1.66005 & -4.962 \\
\hline $\mathrm{H}$ & 2.64483 & 0.96666 & -1.15113 \\
\hline $\mathrm{H}$ & $-\odot .96701$ & -1.30622 & -5.5150 \\
\hline $\mathrm{H}$ & ๑.70912 & -2.42104 & 3.4080 \\
\hline $\mathrm{H}$ & 0.54673 & -0.14763 & 4.57454 \\
\hline $\mathrm{H}$ & -1.52078 & -1.1643 & 3.46614 \\
\hline $\mathrm{H}$ & -3.16658 & ๑. 28268 & 2.38204 \\
\hline C & -2.11325 & 0.31253 & 0.4809 \\
\hline $\mathrm{H}$ & -3.06227 & -1.30742 & 1.58 \\
\hline $\mathrm{H}$ & -1.13519 & 2.19006 & -0.19662 \\
\hline $\mathrm{H}$ & -2.02337 & 2.35873 & 1.34098 \\
\hline $\mathrm{H}$ & -0.97883 & -0.09489 & -1.36605 \\
\hline $\mathrm{H}$ & -1.75741 & -1.53206 & -0.65136 \\
\hline $\mathrm{H}$ & -2.99991 & ๑.54038 & $-\odot .08474$ \\
\hline
\end{tabular}

- $\quad$ TS8

$\begin{array}{cc}\text { C } & -0.49652 \\ \text { C } & -0.31434 \\ \text { C } & 0.02509 \\ \text { C } & -2.02364 \\ \text { C } & -1.84103 \\ \text { C } & -2.36212 \\ \text { C } & 1.53576 \\ \text { H } & -0.24923 \\ \text { H } & 0.0617 \\ \text { H } & -2.40962 \\ \text { H } & -2.09496 \\ \text { C } & -1.69407 \\ \text { H } & -3.44684 \\ \text { C } & 0.35416 \\ \text { C } & 0.17111 \\ \text { H } & 0.1375 \\ \text { H } & -0.17897 \\ \text { H } & -1.95573 \\ \text { H } & -2.0763\end{array}$

1.39023
-1.05037
0.37997
1.24418
-1.19751
-0.18576
0.53235
2.40808
-1.77183
1.97341
-2.21745
-0.45988
-0.29196
-1.32361
1.11464
-2.34841
1.84638
-1.46332
0.24598

$-2.38914$

$-2.97066$

$-3.42924$

$-2.25228$

$-2.83138$

$-1.79249$

$-3.59869$

$-2.70762$

$-3.70393$

$-1.53058$

$-2.52283$

$-0.42794$

$-1.68664$

$-1.60689$

$-1.02537$

$-1.28897$

$-0.28766$

$-0.0807$

๑. 31881 


$\begin{array}{lccc}\mathrm{C} & -0.16165 & -0.32047 & -0.547 \\ \mathrm{P} & 0.71984 & -0.34851 & 1.22191 \\ \mathrm{C} & 1.68429 & 1.28904 & -1.14256 \\ \mathrm{C} & 1.87166 & -1.20646 & -1.73941 \\ \mathrm{C} & 0.28472 & -0.0922 & 2.99969 \\ \mathrm{C} & -1.06131 & 0.10938 & 3.3391 \\ \mathrm{C} & -1.44906 & 0.2791 & 4.66564 \\ \mathrm{C} & -0.49941 & 0.22335 & 5.6833 \\ \mathrm{C} & 1.22746 & -0.14907 & 4.03585 \\ \mathrm{C} & 0.83901 & 0.00189 & 5.36477 \\ \mathrm{H} & -1.81535 & 0.12059 & 2.55969 \\ \mathrm{H} & -2.49418 & 0.4393 & 4.90518 \\ \mathrm{H} & 2.27327 & -0.31388 & 3.8036 \\ \mathrm{H} & 1.58325 & -0.05041 & 6.15104 \\ \mathrm{H} & 2.20226 & -0.77367 & 1.19404 \\ \mathrm{H} & -0.80096 & 0.34354 & 6.71693 \\ \mathrm{H} & -2.32505 & -1.02673 & -3.79958 \\ \mathrm{H} & -2.50882 & 1.45767 & -3.21143 \\ \mathrm{H} & -0.42664 & 0.57954 & -4.40725 \\ \mathrm{H} & 1.76466 & 1.54938 & -3.92378 \\ \mathrm{C} & 2.1386 & 0.24225 & -2.22778 \\ \mathrm{H} & 1.89197 & -0.17821 & -4.34836 \\ \mathrm{H} & 2.14941 & 1.09248 & -0.17397 \\ \mathrm{H} & 1.90968 & 2.31122 & -1.45379 \\ \mathrm{H} & 2.33757 & -1.401 & -0.77092 \\ \mathrm{H} & 2.23104 & -1.93526 & -2.46982 \\ \mathrm{H} & 3.20764 & 0.33944 & -2.30117\end{array}$




\section{X-Ray structures}

The X-ray crystallographic data for 12, 41 and $\mathbf{4 6}$ were collected using $\varphi$ and $\omega$ scans using $\mathrm{MoK}_{\alpha}$ radiation $(\lambda=0.71073 \AA$, X-ray tube, $50 \mathrm{kV}, 32 \mathrm{~mA})$. The total number of runs and images was based on the strategy calculation with the program Collect (Nonius BV, 1997-2000). Cell parameters were retrieved using the SCALEPACK (Otwinowski, 1997) software and refined using DENZO (Otwinowski, 1997). Data reduction was performed using the DENZO software (Otwinowski, 1997) which corrects for Lorentz polarisation.

The X-ray crystallographic data for 19 and 40 were collected using $\mathrm{MoK}_{\alpha}$ radiation (X-ray tube, $50 \mathrm{kV}, 32 \mathrm{~mA}$ ). The total number of runs and images was based on the strategy calculation from the program APEX2. Cell parameters were retrieved and refined using the SAINT (V8.34A, 2013) software. Data reduction was performed using the SAINT (V8.34A, 2013) software which corrects for Lorentz polarisation.

The structure was solved by Direct Methods using the SHELXS97 program structure solution program for 41 and $\mathbf{4 6}$ and SHELXS2013 for 12, 19 and 40 and refined by Least Squares using version of the ShelXL (Sheldrick, 2008). All non-hydrogen atoms were refined anisotropically. $\mathrm{C}$-H hydrogen atom positions were calculated geometrically and refined using the riding model. $\mathrm{O}-\mathrm{H}$ hydrogen atom was refined as a rotating group.

Supplementary crystallographic data was deposited to the Cambridge Crystallographic Data Centre with the deposition numbers CCDC 10545651054569. These data can be obtained free of charge via https://summary.ccdc.cam.ac.uk/structure-summary-form.

The X-ray crystallographic data for 17, 42,43 and 44 were collected using $\mathrm{MoK}_{\alpha}$ radiation with wavelength $0.71073 \AA$ and a graphite monochromator were used. The PLATON MULABS semi-empirical absorption correction using multiple scanned reflection was applied on the data and the structure was solved for 17 and 44 by Direct Methods in SHELXS97 and for $\mathbf{4 2}$ and $\mathbf{4 3}$ by intrinsic phasing using SHELXT2014 and refined using full matrix least squares with SHELXL2014 for 17, 42, 43 and with SHELXL2012 for 44. All non-hydrogen atoms were refined anisotropically, $\mathrm{C}-\mathrm{H}$ hydrogen atoms were positioned geometrically and $\mathrm{O}-\mathrm{H}$ hydrogen atoms were located in difference map and refined isotropically.

For data collection of 13, 21 and $24 \mathrm{MoK}_{\alpha}$ radiation with wavelength $0.71073 \AA$ and a collimating multilayer mirror were used. Semi-empirical absorption correction from equivalents was applied using SADABS-2014/4 and the structure was solved by intrinsic phasing using SHELXT2014. Refinement was performed against $\mathrm{F}^{2}$ using SHELXL-2014/7. All non-hydrogen atoms were refined anisotropically, C-H hydrogen atoms were positioned geometrically and $\mathrm{O}-\mathrm{H}$ hydrogen atoms were located in difference map and refined isotropically.

Supplementary crystallographic data was deposited to the Cambridge Crystallographic Data Centre with the deposition numbers CCDC 1053886-1053892. These data can be obtained free of charge via https://summary.ccdc.cam.ac.uk/structure-summary-form. 


\section{(12) (9-Chlorodiamant-4-yl)phosphonic dichloride}

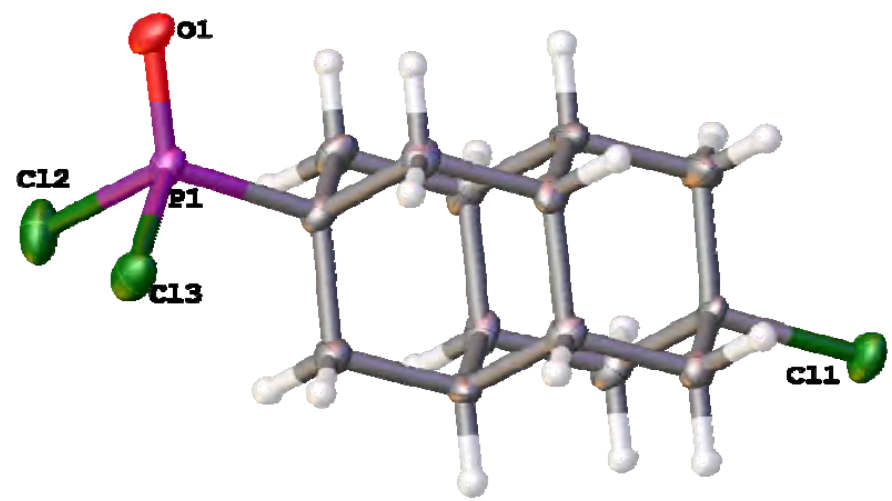

Ellipsoids are drawn at $50 \%$ probability.

Experimental. A single colourless needle-shaped crystalsuitable crystal (Compound 12) was selected and mounted on a diffractometer. The crystal $(0.25 \times 0.07 \times$ $0.05 \mathrm{~mm}^{3}$ ) was kept at $T=115 \mathrm{~K}$ during data collection. Using Olex2 (Dolomanov et al., 2009), the structure was solved with the ShelXS (Sheldrick, 2013) structure solution program, using the Direct Methods solution method. The model was refined with version 2013-3 of XL (Sheldrick, 2008) using Least Squares minimisation.

Crystal Data. $\mathrm{C}_{14} \mathrm{H}_{18} \mathrm{Cl}_{3} \mathrm{OP}, M_{r}=339.60$, monoclinic, $\mathrm{P} 2{ }_{1}$ (No. 4), $a=7.2710$ (5) $\AA, b=8.3207$ (4) $\AA, c=12.3475(8)$ $\AA$, $\beta=102.708(4)^{\circ}, \alpha=\gamma=90^{\circ}, V=728.72(8) \AA^{3}, T=$ $115 \mathrm{~K}, Z=2, Z^{\prime}=1, \mu\left(\mathrm{MoK}_{\alpha}\right)=0.727,8997$ reflections measured, 3780 unique $\left(R_{\text {int }}=0.0421\right)$ which were used in all calculations. The final $w R_{2}$ was 0.0781 (all data) and $R_{1}$ was 0.0412 (I $>2(\mathrm{I})$ ).

\begin{tabular}{|c|c|}
\hline Compound & Compound 1 \\
\hline $\mathrm{N}^{\circ} \mathrm{CCDC}$ & 1054565 \\
\hline Formula & $\mathrm{C}_{14} \mathrm{H}_{18} \mathrm{Cl}_{3} \mathrm{OP}$ \\
\hline$D_{\text {calc. }} / \mathrm{g} \mathrm{cm}^{-3}$ & 1.548 \\
\hline$\mu / \mathrm{mm}^{-1}$ & 0.727 \\
\hline Formula Weight & 339.60 \\
\hline Colour & colourless \\
\hline Shape & needle \\
\hline Max Size/mm & 0.25 \\
\hline Mid Size/mm & 0.07 \\
\hline Min Size/mm & 0.05 \\
\hline$T / \mathrm{K}$ & 115 \\
\hline Crystal System & monoclinic \\
\hline Flack Parameter & $0.04(6)$ \\
\hline Hooft Parameter & $0.01(5)$ \\
\hline Space Group & $\mathrm{P} 2_{1}$ \\
\hline$a / \AA ̊$ & $7.2710(5)$ \\
\hline$b / \AA$ & $8.3207(4)$ \\
\hline$c / \AA ̊$ & $12.3475(8)$ \\
\hline$\alpha /^{\circ}$ & 90 \\
\hline$\beta 1^{\circ}$ & $102.708(4)$ \\
\hline$\left.\gamma\right|^{\circ}$ & 90 \\
\hline $\mathrm{V} / \AA^{3}$ & $728.72(8)$ \\
\hline$Z$ & 2 \\
\hline$Z^{\prime}$ & 1 \\
\hline$\Theta_{\min } /^{\circ}$ & 2.995 \\
\hline$\left.\Theta_{\max }\right|^{\circ}$ & 29.207 \\
\hline Measured Refl. & 8997 \\
\hline Independent Refl. & 3780 \\
\hline Reflections Used & 2939 \\
\hline$R_{\text {int }}$ & 0.0421 \\
\hline Parameters & 172 \\
\hline Restraints & 1 \\
\hline Largest Peak & 0.366 \\
\hline Deepest Hole & -0.338 \\
\hline GooF & 0.986 \\
\hline$w R_{2}$ (all data) & 0.0781 \\
\hline$w R_{2}$ & 0.0699 \\
\hline$R_{1}$ (all data) & 0.0680 \\
\hline$R_{1}$ & 0.0412 \\
\hline
\end{tabular}


Experimental Extended. A colourless needle-shaped crystal with dimensions $0.25 \times 0.07 \times 0.05 \mathrm{~mm}^{3}$ was mounted on a mylar loop with grease. Cell parameters were retrieved using the SCALEPACK (Otwinowski, 1997) software and refined using DENZO (Otwinowski, 1997)on 2417 reflections, 27 of the observed reflections. Data reduction was performed using the DENZO software (Otwinowski, 1997) which corrects for Lorentz polarization. The final completeness is 99.70 out to 29.207 in $\Theta$. The absorption coefficient (MU) of this material is 0.727 and the minimum and maximum transmissions are 0.6415 and 0.7458.The structure was solved by Direct Methods using the ShelXS (Sheldrick, 2013) structure solution program and refined by Least Squares using version 2013-3 of XL (Sheldrick, 2008).The structure was solved in the space group $P 2_{1}$ (\# 4). All non-hydrogen atoms were refined anisotropically. Hydrogen atom positions were calculated geometrically and refined using the riding model.

Table S2: Fractional Atomic Coordinates $\left(\times 10^{4}\right)$ and Equivalent Isotropic Displacement Parameters $\left(\AA^{2} \times 10^{3}\right)$ for Compound 12. $U_{e q}$ is defined as $1 / 3$ of of the trace of the orthogonalised $U_{i j}$.

\begin{tabular}{lcccc}
\hline Atom & $\mathbf{x}$ & $\mathrm{y}$ & $\mathbf{z}$ & $U_{e q}$ \\
\hline C1 & $6465(5)$ & $3615(4)$ & $8096(3)$ & $15.5(8)$ \\
C2 & $4651(5)$ & $4315(5)$ & $7359(3)$ & $19.0(9)$ \\
C3 & $5916(6)$ & $2514(4)$ & $8978(3)$ & $20.2(9)$ \\
C4 & $7555(5)$ & $2652(5)$ & $7388(3)$ & $19.8(9)$ \\
C5 & $6307(5)$ & $1284(5)$ & $6811(3)$ & $17.9(8)$ \\
C6 & $4492(5)$ & $1935(4)$ & $6071(3)$ & $17.4(9)$ \\
C7 & $3431(5)$ & $2923(5)$ & $6785(3)$ & $18.7(9)$ \\
C8 & $2886(5)$ & $1794(5)$ & $7645(3)$ & $21.9(9)$ \\
C9 & $4697(6)$ & $1134(4)$ & $8390(3)$ & $20.5(9)$ \\
C10 & $5765(6)$ & $165(4)$ & $7669(3)$ & $19.7(9)$ \\
C11 & $3267(5)$ & $567(4)$ & $5489(3)$ & $17.8(9)$ \\
C12 & $1650(6)$ & $414(5)$ & $7060(4)$ & $26.6(10)$ \\
C13 & $4517(6)$ & $-1219(4)$ & $7098(3)$ & $22.3(10)$ \\
C14 & $2755(5)$ & $-513(4)$ & $6365(3)$ & $15.9(8)$ \\
O1 & $6838(4)$ & $6423(3)$ & $9360(2)$ & $24.7(6)$ \\
P1 & $7831.3(15)$ & $5292.5(11)$ & $8796.0(9)$ & $17.6(2)$ \\
Cl1 & $1314.1(15)$ & $-2157.4(12)$ & $5658.5(9)$ & $27.2(3)$ \\
Cl2 & $10102.4(15)$ & $4297.7(13)$ & $9807.1(9)$ & $32.8(3)$ \\
Cl3 & $9023.1(15)$ & $6377.8(11)$ & $7660.1(8)$ & $26.7(3)$
\end{tabular}

Table S3: Anisotropic Displacement Parameters $\left(\times 10^{4}\right)$ for Compound 12. The anisotropic displacement factor exponent takes the form: $-2 \pi^{2}\left[a^{* 2} \times U_{11}+\ldots 2 h k a^{*} \times b^{*} \times U_{12}\right]$

\begin{tabular}{llllrrr}
\hline Atom & \multicolumn{1}{c}{$U_{11}$} & \multicolumn{1}{c}{$U_{22}$} & \multicolumn{1}{c}{$U_{33}$} & $U_{23}$ & $U_{13}$ & $U_{12}$ \\
\hline C1 & $19(2)$ & $11.3(17)$ & $15(2)$ & $-1.5(15)$ & $1.2(17)$ & $-0.4(15)$ \\
C2 & $19(2)$ & $13.8(17)$ & $22(2)$ & $0.9(17)$ & $-0.7(17)$ & $2.5(17)$ \\
C3 & $29(2)$ & $19(2)$ & $13(2)$ & $0.1(15)$ & $5.3(18)$ & $-3.5(17)$ \\
C4 & $18(2)$ & $18.8(19)$ & $23(2)$ & $-5.0(18)$ & $5.5(18)$ & $-0.9(17)$ \\
C5 & $12.9(19)$ & $16.3(18)$ & $25(2)$ & $-8.1(18)$ & $5.5(16)$ & $0.3(16)$ \\
C6 & $23(2)$ & $13.6(17)$ & $16(2)$ & $1.4(15)$ & $5.6(17)$ & $-1.8(16)$ \\
C7 & $16(2)$ & $15.1(18)$ & $22(2)$ & $-3.3(17)$ & $-2.6(17)$ & $4.7(16)$ \\
C8 & $20(2)$ & $22(2)$ & $27(2)$ & $-10.3(18)$ & $13.0(19)$ & $-7.3(18)$ \\
C9 & $31(2)$ & $17(2)$ & $14(2)$ & $-0.6(16)$ & $7.2(18)$ & $-11.3(18)$ \\
C10 & $23(2)$ & $11.2(17)$ & $21(2)$ & $-0.5(17)$ & $-2.4(18)$ & $-1.3(17)$ \\
C11 & $18(2)$ & $16(2)$ & $19(2)$ & $-1.7(16)$ & $2.0(17)$ & $0.3(16)$ \\
C12 & $21(2)$ & $30(2)$ & $32(3)$ & $-13(2)$ & $14.6(19)$ & $-9(2)$ \\
C13 & $32(3)$ & $14.1(19)$ & $21(2)$ & $-1.5(17)$ & $4.2(19)$ & $-5.1(17)$ \\
C14 & $19(2)$ & $14.7(17)$ & $15(2)$ & $-5.6(16)$ & $5.1(16)$ & $-7.1(16)$ \\
01 & $31.2(16)$ & $18.9(14)$ & $26.3(16)$ & $-9.2(13)$ & $11.5(13)$ & $-7.0(13)$ \\
P1 & $20.5(6)$ & $14.9(5)$ & $17.2(6)$ & $-1.9(4)$ & $3.3(4)$ & $-2.9(4)$ \\
C11 & $30.0(6)$ & $24.4(5)$ & $27.3(6)$ & $-8.7(4)$ & $6.3(5)$ & $-13.1(5)$ \\
C12 & $29.6(6)$ & $25.7(5)$ & $34.6(7)$ & $-0.5(5)$ & $-11.0(5)$ & $-3.2(5)$
\end{tabular}




\begin{tabular}{lrrrrrr}
\hline Atom & $U_{11}$ & $U_{22}$ & $U_{33}$ & $U_{23}$ & $U_{13}$ & $U_{12}$ \\
\hline $\mathrm{Cl} 3$ & $32.6(6)$ & $23.9(5)$ & $25.7(6)$ & $-3.4(4)$ & $11.0(5)$ & $-12.1(4)$
\end{tabular}

Table S4: Bond Lengths in Å for Compound 12.

\begin{tabular}{lll}
\hline Atom & Atom & Length/ \\
\hline C1 & C2 & $1.543(5)$ \\
C1 & C3 & $1.541(5)$ \\
C1 & C4 & $1.529(5)$ \\
C1 & P1 & $1.818(4)$ \\
C2 & C7 & $1.535(5)$ \\
C3 & C9 & $1.532(5)$ \\
C4 & C5 & $1.530(5)$ \\
C5 & C6 & $1.529(5)$ \\
C5 & C10 & $1.525(5)$ \\
C6 & C7 & $1.532(5)$ \\
C6 & C11 & $1.525(5)$ \\
C7 & C8 & $1.533(6)$
\end{tabular}

\begin{tabular}{lll}
\hline Atom & Atom & Length/ \\
\hline C8 & C9 & $1.532(6)$ \\
C8 & C12 & $1.537(5)$ \\
C9 & C10 & $1.534(5)$ \\
C10 & C13 & $1.538(5)$ \\
C11 & C14 & $1.515(5)$ \\
C12 & C14 & $1.510(5)$ \\
C13 & C14 & $1.515(5)$ \\
C14 & Cl1 & $1.825(4)$ \\
01 & P1 & $1.453(3)$ \\
P1 & Cl2 & $2.0159(14)$ \\
P1 & Cl3 & $2.0170(14)$
\end{tabular}

Table S5: Bond Angles in ${ }^{\circ}$ for Compound 12.

\begin{tabular}{|c|c|c|c|}
\hline Atom & Atom & Atom & Angle/ ${ }^{\circ}$ \\
\hline$\overline{\mathrm{C} 2}$ & $\mathrm{C} 1$ & P1 & $107.3(3)$ \\
\hline C3 & $\mathrm{C} 1$ & $\mathrm{C} 2$ & 108.7(3) \\
\hline C3 & $\mathrm{C} 1$ & P1 & $108.7(3)$ \\
\hline $\mathrm{C} 4$ & $\mathrm{C} 1$ & $\mathrm{C} 2$ & $110.1(3)$ \\
\hline $\mathrm{C} 4$ & $\mathrm{C} 1$ & $\mathrm{C} 3$ & $109.8(3)$ \\
\hline $\mathrm{C} 4$ & $\mathrm{C} 1$ & $\mathrm{P} 1$ & $112.1(3)$ \\
\hline $\mathrm{C} 7$ & $\mathrm{C} 2$ & $\mathrm{C} 1$ & $108.6(3)$ \\
\hline C9 & $\mathrm{C} 3$ & $\mathrm{C} 1$ & $108.9(3)$ \\
\hline C1 & $\mathrm{C} 4$ & $\mathrm{C} 5$ & $109.1(3)$ \\
\hline C6 & C5 & $\mathrm{C} 4$ & 111.1(3) \\
\hline C10 & $\mathrm{C} 5$ & $\mathrm{C} 4$ & $110.4(3)$ \\
\hline C10 & $\mathrm{C} 5$ & $\mathrm{C} 6$ & 108.1(3) \\
\hline C5 & C6 & $\mathrm{C} 7$ & $108.9(3)$ \\
\hline C11 & C6 & $\mathrm{C} 5$ & $110.8(3)$ \\
\hline C11 & C6 & $\mathrm{C} 7$ & $110.8(3)$ \\
\hline C6 & $\mathrm{C} 7$ & $\mathrm{C} 2$ & $110.9(3)$ \\
\hline C6 & $\mathrm{C} 7$ & $\mathrm{C} 8$ & $108.0(3)$ \\
\hline C8 & $\mathrm{C} 7$ & $\mathrm{C} 2$ & $110.7(3)$ \\
\hline $\mathrm{C} 7$ & C8 & $\mathrm{C} 12$ & $110.2(3)$ \\
\hline C9 & C8 & $\mathrm{C} 7$ & $108.4(3)$ \\
\hline C9 & C8 & $\mathrm{C} 12$ & $110.6(3)$ \\
\hline C3 & C9 & C8 & $110.3(3)$ \\
\hline C3 & C9 & C10 & $110.8(3)$ \\
\hline C8 & C9 & C10 & $108.5(3)$ \\
\hline $\mathrm{C} 5$ & C10 & C9 & $108.9(3)$ \\
\hline $\mathrm{C} 5$ & C10 & C13 & $110.8(3)$ \\
\hline C9 & C10 & C13 & $109.7(3)$ \\
\hline C14 & C11 & C6 & $108.4(3)$ \\
\hline C14 & $\mathrm{C} 12$ & $\mathrm{C} 8$ & $108.6(3)$ \\
\hline C14 & C13 & C10 & 108.6(3) \\
\hline C11 & C14 & $\mathrm{Cl} 1$ & $108.0(2)$ \\
\hline C12 & C14 & C11 & $110.3(3)$ \\
\hline C12 & C14 & C13 & $110.2(3)$ \\
\hline C12 & C14 & $\mathrm{Cl} 1$ & $109.5(3)$ \\
\hline C13 & $\mathrm{C} 14$ & C11 & $110.4(3)$ \\
\hline C13 & C14 & $\mathrm{Cl} 1$ & $108.4(3)$ \\
\hline C1 & P1 & $\mathrm{Cl} 2$ & $105.46(13)$ \\
\hline C1 & $\mathrm{P} 1$ & $\mathrm{Cl} 3$ & $106.67(14)$ \\
\hline
\end{tabular}

\begin{tabular}{llll}
\hline Atom & Atom & Atom & Angle $^{\circ}$ \\
\hline O1 & P1 & C1 & $116.58(17)$ \\
O1 & P1 & Cl2 & $113.26(12)$ \\
O1 & P1 & Cl3 & $112.20(12)$ \\
Cl2 & P1 & Cl3 & $101.29(6)$
\end{tabular}


Table S6: Hydrogen Fractional Atomic Coordinates $\left(\times 10^{4}\right)$ and Equivalent Isotropic Displacement Parameters $\left(\AA^{2} \times 10^{3}\right)$ for Compound 12. $U_{e q}$ is defined as $1 / 3$ of of the trace of the orthogonalised $U_{i j}$.

\begin{tabular}{|c|c|c|c|c|c|}
\hline Atom & & & & & $U_{e q}$ \\
\hline $\mathrm{H} 2 \mathrm{~A}$ & 3948 & 4938 & 7819 & 23 & \\
\hline H2B & 4975 & 5045 & 6796 & 23 & \\
\hline H3A & 7065 & 2078 & 9474 & 24 & \\
\hline H3B & 5205 & 3135 & 9434 & 24 & \\
\hline $\mathrm{H} 4 \mathrm{~A}$ & 8718 & 2206 & 7864 & 24 & \\
\hline H4B & 7918 & 3361 & 6827 & 24 & \\
\hline H5 & 7017 & 659 & 6345 & 21 & \\
\hline H6 & 4831 & 2655 & 5496 & 21 & \\
\hline H7 & 2257 & 3369 & 6300 & 22 & \\
\hline H8 & 2167 & 2413 & 8110 & 26 & \\
\hline H9 & 4357 & 407 & 8961 & 25 & \\
\hline H10 & 6937 & -290 & 8150 & 24 & \\
\hline H11A & 2110 & 1005 & 5002 & 21 & \\
\hline H11B & 3964 & -53 & 5024 & 21 & \\
\hline H12A & 1289 & -305 & 7617 & 32 & \\
\hline H12B & 484 & 852 & 6581 & 32 & \\
\hline H13A & 5212 & -1862 & 6644 & 27 & \\
\hline H13B & 4173 & -1933 & 7663 & 27 & \\
\hline
\end{tabular}


(13) (4,9-Diamantyl)diphosphonic dichloride

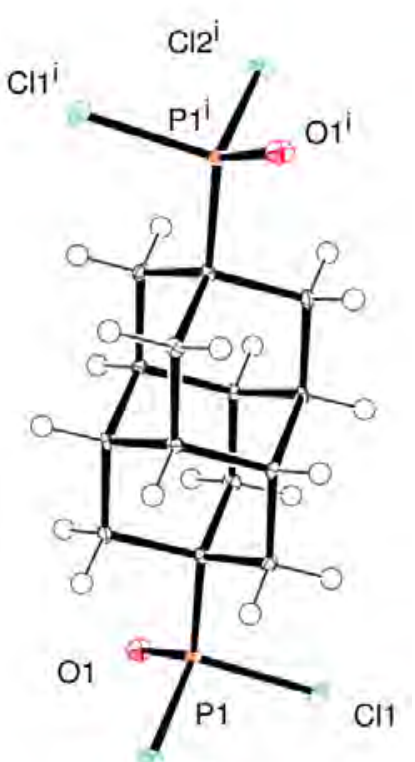

$\mathrm{Cl} 2$

Ellipsoids are drawn at $50 \%$ probability.

Table S7. Crystal data and structure refinement for Compound 13.

CCDC No.

Empirical formula

Formula weight

Temperature

Wavelength

Crystal system

Space group

Unit cell dimensions

Volume

Z

Density (calculated)

Absorption coefficient

$\mathrm{F}(000)$

Crystal size

Theta range for data collection

Index ranges

Reflections collected

Independent reflections

Completeness to theta $=25.242^{\circ}$

Absorption correction
1053886

C14 H18 Cl4 O2 P2

422.02

100(2) K

$0.71073 \AA$

Triclinic

$\mathrm{P}-1$

$\mathrm{a}=6.3875(5) \AA \quad \alpha=95.801(2)^{\circ}$.

$\mathrm{b}=8.1583(7) \AA \quad \beta=108.129(2)^{\circ}$.

$\mathrm{c}=9.1125(7) \AA \quad \gamma=111.841(2)^{\circ}$.

406.04(6) $\AA^{3}$

1

$1.726 \mathrm{Mg} / \mathrm{m}^{3}$

$0.928 \mathrm{~mm}^{-1}$

216

$0.161 \times 0.065 \times 0.037 \mathrm{~mm}^{3}$

2.426 to $26.732^{\circ}$.

$-8<=\mathrm{h}<=8,-10<=\mathrm{k}<=10,-11<=\mathrm{l}<=11$

17659

$1716[\mathrm{R}(\mathrm{int})=0.0369]$

$99.7 \%$

Empirical 
Max. and min. transmission

Refinement method

Data / restraints / parameters

Goodness-of-fit on $\mathrm{F}^{2}$

Final $\mathrm{R}$ indices $[\mathrm{I}>2 \operatorname{sigma}(\mathrm{I})]$

$\mathrm{R}$ indices (all data)

Extinction coefficient

Largest diff. peak and hole
0.7454 and 0.6708

Full-matrix least-squares on $\mathrm{F}^{2}$

$1716 / 0 / 100$

1.532

$\mathrm{R} 1=0.0338, \mathrm{wR} 2=0.1060$

$\mathrm{R} 1=0.0354, \mathrm{wR} 2=0.1066$

$\mathrm{n} / \mathrm{a}$

0.453 and -0.480 e. $\AA^{-3}$ 
Table S8. Bond lengths $[\AA]$ and angles $\left[^{\circ}\right]$ for Compound 13.

\begin{tabular}{|c|c|}
\hline $\mathrm{Cl}(1)-\mathrm{P}(1)$ & $2.0208(12)$ \\
\hline$C(1)-C(6)$ & $1.534(4)$ \\
\hline$C(1)-C(2)$ & $1.542(4)$ \\
\hline$C(1)-C(7) \# 1$ & $1.550(4)$ \\
\hline $\mathrm{C}(1)-\mathrm{P}(1)$ & $1.813(3)$ \\
\hline $\mathrm{O}(1)-\mathrm{P}(1)$ & $1.463(2)$ \\
\hline $\mathrm{P}(1)-\mathrm{Cl}(2)$ & $2.0237(12)$ \\
\hline$C(2)-C(3)$ & $1.534(4)$ \\
\hline $\mathrm{C}(2)-\mathrm{H}(2 \mathrm{~A})$ & 0.9900 \\
\hline $\mathrm{C}(2)-\mathrm{H}(2 \mathrm{~B})$ & 0.9900 \\
\hline$C(5)-C(4)$ & $1.534(4)$ \\
\hline$C(5)-C(6)$ & $1.534(4)$ \\
\hline$C(5)-C(3) \# 1$ & $1.541(4)$ \\
\hline $\mathrm{C}(5)-\mathrm{H}(5)$ & 1.0000 \\
\hline$C(4)-C(7)$ & $1.535(4)$ \\
\hline$C(4)-C(3)$ & $1.542(4)$ \\
\hline $\mathrm{C}(4)-\mathrm{H}(4)$ & 1.0000 \\
\hline$C(3)-C(5) \# 1$ & $1.541(4)$ \\
\hline $\mathrm{C}(3)-\mathrm{H}(3)$ & 1.0000 \\
\hline $\mathrm{C}(7)-\mathrm{C}(1) \# 1$ & $1.550(4)$ \\
\hline $\mathrm{C}(7)-\mathrm{H}(7 \mathrm{~A})$ & 0.9900 \\
\hline $\mathrm{C}(7)-\mathrm{H}(7 \mathrm{~B})$ & 0.9900 \\
\hline $\mathrm{C}(6)-\mathrm{H}(6 \mathrm{~A})$ & 0.9900 \\
\hline $\mathrm{C}(6)-\mathrm{H}(6 \mathrm{~B})$ & 0.9900 \\
\hline $\mathrm{C}(6)-\mathrm{C}(1)-\mathrm{C}(2)$ & $110.0(3)$ \\
\hline$C(6)-C(1)-C(7) \# 1$ & $109.5(3)$ \\
\hline $\mathrm{C}(2)-\mathrm{C}(1)-\mathrm{C}(7) \# 1$ & 109.1(3) \\
\hline $\mathrm{C}(6)-\mathrm{C}(1)-\mathrm{P}(1)$ & $113.4(2)$ \\
\hline$C(2)-C(1)-P(1)$ & $107.5(2)$ \\
\hline $\mathrm{C}(7) \# 1-\mathrm{C}(1)-\mathrm{P}(1)$ & $107.1(2)$ \\
\hline $\mathrm{O}(1)-\mathrm{P}(1)-\mathrm{C}(1)$ & $116.26(15)$ \\
\hline $\mathrm{O}(1)-\mathrm{P}(1)-\mathrm{Cl}(1)$ & $112.51(11)$ \\
\hline $\mathrm{C}(1)-\mathrm{P}(1)-\mathrm{Cl}(1)$ & $106.25(11)$ \\
\hline $\mathrm{O}(1)-\mathrm{P}(1)-\mathrm{Cl}(2)$ & $112.10(11)$ \\
\hline $\mathrm{C}(1)-\mathrm{P}(1)-\mathrm{Cl}(2)$ & $106.49(11)$ \\
\hline $\mathrm{Cl}(1)-\mathrm{P}(1)-\mathrm{Cl}(2)$ & $102.05(5)$ \\
\hline $\mathrm{C}(3)-\mathrm{C}(2)-\mathrm{C}(1)$ & 109.2(3) \\
\hline $\mathrm{C}(3)-\mathrm{C}(2)-\mathrm{H}(2 \mathrm{~A})$ & 109.8 \\
\hline
\end{tabular}




\begin{tabular}{|c|c|}
\hline $\mathrm{C}(1)-\mathrm{C}(2)-\mathrm{H}(2 \mathrm{~A})$ & 109.8 \\
\hline $\mathrm{C}(3)-\mathrm{C}(2)-\mathrm{H}(2 \mathrm{~B})$ & 109.8 \\
\hline $\mathrm{C}(1)-\mathrm{C}(2)-\mathrm{H}(2 \mathrm{~B})$ & 109.8 \\
\hline $\mathrm{H}(2 \mathrm{~A})-\mathrm{C}(2)-\mathrm{H}(2 \mathrm{~B})$ & 108.3 \\
\hline$C(4)-C(5)-C(6)$ & $110.2(3)$ \\
\hline$C(4)-C(5)-C(3) \# 1$ & $108.5(3)$ \\
\hline$C(6)-C(5)-C(3) \# 1$ & $110.5(3)$ \\
\hline $\mathrm{C}(4)-\mathrm{C}(5)-\mathrm{H}(5)$ & 109.2 \\
\hline $\mathrm{C}(6)-\mathrm{C}(5)-\mathrm{H}(5)$ & 109.2 \\
\hline $\mathrm{C}(3) \# 1-\mathrm{C}(5)-\mathrm{H}(5)$ & 109.2 \\
\hline$C(5)-C(4)-C(7)$ & 111.1(3) \\
\hline$C(5)-C(4)-C(3)$ & $109.3(3)$ \\
\hline$C(7)-C(4)-C(3)$ & $110.0(3)$ \\
\hline $\mathrm{C}(5)-\mathrm{C}(4)-\mathrm{H}(4)$ & 108.8 \\
\hline $\mathrm{C}(7)-\mathrm{C}(4)-\mathrm{H}(4)$ & 108.8 \\
\hline $\mathrm{C}(3)-\mathrm{C}(4)-\mathrm{H}(4)$ & 108.8 \\
\hline$C(2)-C(3)-C(5) \# 1$ & $110.0(3)$ \\
\hline$C(2)-C(3)-C(4)$ & $110.5(3)$ \\
\hline$C(5) \# 1-C(3)-C(4)$ & $108.4(2)$ \\
\hline $\mathrm{C}(2)-\mathrm{C}(3)-\mathrm{H}(3)$ & 109.3 \\
\hline $\mathrm{C}(5) \# 1-\mathrm{C}(3)-\mathrm{H}(3)$ & 109.3 \\
\hline $\mathrm{C}(4)-\mathrm{C}(3)-\mathrm{H}(3)$ & 109.3 \\
\hline $\mathrm{C}(4)-\mathrm{C}(7)-\mathrm{C}(1) \# 1$ & $108.6(3)$ \\
\hline $\mathrm{C}(4)-\mathrm{C}(7)-\mathrm{H}(7 \mathrm{~A})$ & 110.0 \\
\hline $\mathrm{C}(1) \# 1-\mathrm{C}(7)-\mathrm{H}(7 \mathrm{~A})$ & 110.0 \\
\hline $\mathrm{C}(4)-\mathrm{C}(7)-\mathrm{H}(7 \mathrm{~B})$ & 110.0 \\
\hline $\mathrm{C}(1) \# 1-\mathrm{C}(7)-\mathrm{H}(7 \mathrm{~B})$ & 110.0 \\
\hline $\mathrm{H}(7 \mathrm{~A})-\mathrm{C}(7)-\mathrm{H}(7 \mathrm{~B})$ & 108.4 \\
\hline$C(1)-C(6)-C(5)$ & 109.2(3) \\
\hline $\mathrm{C}(1)-\mathrm{C}(6)-\mathrm{H}(6 \mathrm{~A})$ & 109.8 \\
\hline$C(5)-C(6)-H(6 A)$ & 109.8 \\
\hline $\mathrm{C}(1)-\mathrm{C}(6)-\mathrm{H}(6 \mathrm{~B})$ & 109.8 \\
\hline $\mathrm{C}(5)-\mathrm{C}(6)-\mathrm{H}(6 \mathrm{~B})$ & 109.8 \\
\hline $\mathrm{H}(6 \mathrm{~A})-\mathrm{C}(6)-\mathrm{H}(6 \mathrm{~B})$ & 108.3 \\
\hline
\end{tabular}

Symmetry transformations used to generate equivalent atoms:

$\# 1-\mathrm{x}+1,-\mathrm{y}+1,-\mathrm{z}+1$ 


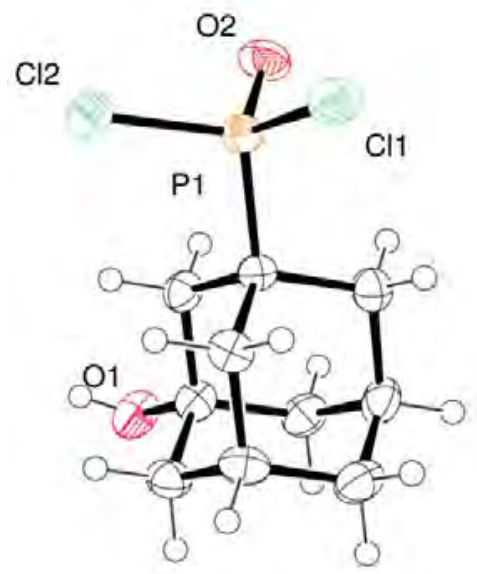

Ellipsoids are drawn at $50 \%$ probability.

Table S9. Crystal data and structure refinement for Compound 17.

CCDC No.

Empirical formula

Formula weight

Temperature

Wavelength

Crystal system

Space group

Unit cell dimensions

Volume

Z

Density (calculated)

Absorption coefficient

$\mathrm{F}(000)$

Crystal size

Theta range for data collection

Index ranges

Reflections collected

Independent reflections

Completeness to theta $=25.242^{\circ}$

Absorption correction

Max. and min. transmission
1053891

C20 H30 Cl4 O4 P2

538.18

150(2) K

$0.71073 \AA$

Orthorhombic

Pbca

$\mathrm{a}=17.865(4) \AA$ $\alpha=90^{\circ}$.

$\mathrm{b}=11.720(2) \AA$

$\beta=90^{\circ}$.

$\mathrm{c}=22.556(5) \AA$ $\gamma=90^{\circ}$.

4722.7(16) $\AA^{3}$

8

$1.514 \mathrm{Mg} / \mathrm{m}^{3}$

$0.662 \mathrm{~mm}^{-1}$

2240

$0.450 \times 0.120 \times 0.050 \mathrm{~mm}^{3}$

1.806 to $27.476^{\circ}$.

$-23<=\mathrm{h}<=22,-15<=\mathrm{k}<=15,-29<=1<=28$

42127

$5405[\mathrm{R}($ int $)=0.0806]$

$100.0 \%$

Empirical

0.76884 and 0.72322 
Refinement method

Data / restraints / parameters

Goodness-of-fit on $\mathrm{F}^{2}$

Final $\mathrm{R}$ indices $[\mathrm{I}>2 \operatorname{sigma}(\mathrm{I})]$

$\mathrm{R}$ indices (all data)

Extinction coefficient

Largest diff. peak and hole
Full-matrix least-squares on $\mathrm{F}^{2}$

$5405 / 0 / 275$

1.029

$\mathrm{R} 1=0.0500, \mathrm{wR} 2=0.1220$

$\mathrm{R} 1=0.0837, \mathrm{wR} 2=0.1339$

$\mathrm{n} / \mathrm{a}$

1.057 and -0.359 e. $\AA^{-3}$

Table S10. Bond lengths $[\AA]$ and angles $\left[{ }^{\circ}\right]$ for Compound 17.

\begin{tabular}{|c|c|}
\hline $\mathrm{Cl}(1)-\mathrm{P}(1)$ & $2.0079(11)$ \\
\hline $\mathrm{P}(1)-\mathrm{O}(2)$ & $1.472(2)$ \\
\hline $\mathrm{P}(1)-\mathrm{C}(1)$ & $1.814(3)$ \\
\hline $\mathrm{P}(1)-\mathrm{Cl}(2)$ & $2.0193(11)$ \\
\hline $\mathrm{O}(1)-\mathrm{C}(3)$ & $1.429(3)$ \\
\hline $\mathrm{O}(1)-\mathrm{H}(1)$ & $0.80(4)$ \\
\hline $\mathrm{C}(1)-\mathrm{C}(9)$ & $1.543(4)$ \\
\hline$C(1)-C(8)$ & $1.549(4)$ \\
\hline$C(1)-C(2)$ & $1.549(4)$ \\
\hline $\mathrm{P}(2)-\mathrm{O}(4)$ & $1.468(2)$ \\
\hline $\mathrm{P}(2)-\mathrm{C}(11)$ & $1.810(3)$ \\
\hline $\mathrm{P}(2)-\mathrm{Cl}(3)$ & $2.0099(11)$ \\
\hline $\mathrm{P}(2)-\mathrm{Cl}(4)$ & $2.0135(11)$ \\
\hline$C(2)-C(3)$ & $1.540(4)$ \\
\hline $\mathrm{C}(2)-\mathrm{H}(2 \mathrm{~A})$ & 0.9900 \\
\hline $\mathrm{C}(2)-\mathrm{H}(2 \mathrm{~B})$ & 0.9900 \\
\hline $\mathrm{O}(3)-\mathrm{C}(13)$ & $1.423(3)$ \\
\hline $\mathrm{O}(3)-\mathrm{H}(3)$ & $0.81(4)$ \\
\hline$C(3)-C(10)$ & $1.518(4)$ \\
\hline$C(3)-C(4)$ & $1.529(4)$ \\
\hline$C(4)-C(5)$ & $1.533(4)$ \\
\hline $\mathrm{C}(4)-\mathrm{H}(4 \mathrm{~A})$ & 0.9900 \\
\hline $\mathrm{C}(4)-\mathrm{H}(4 \mathrm{~B})$ & 0.9900 \\
\hline$C(5)-C(6)$ & $1.528(4)$ \\
\hline$C(5)-C(9)$ & $1.537(4)$ \\
\hline $\mathrm{C}(5)-\mathrm{H}(5)$ & 1.0000 \\
\hline$C(6)-C(7)$ & $1.538(4)$ \\
\hline $\mathrm{C}(6)-\mathrm{H}(6 \mathrm{~A})$ & 0.9900 \\
\hline $\mathrm{C}(6)-\mathrm{H}(6 \mathrm{~B})$ & 0.9900 \\
\hline$C(7)-C(10)$ & $1.531(4)$ \\
\hline
\end{tabular}




\begin{tabular}{|c|c|}
\hline$C(7)-C(8)$ & $1.533(4)$ \\
\hline $\mathrm{C}(7)-\mathrm{H}(7)$ & 1.0000 \\
\hline $\mathrm{C}(8)-\mathrm{H}(8 \mathrm{~A})$ & 0.9900 \\
\hline $\mathrm{C}(8)-\mathrm{H}(8 \mathrm{~B})$ & 0.9900 \\
\hline $\mathrm{C}(9)-\mathrm{H}(9 \mathrm{~A})$ & 0.9900 \\
\hline $\mathrm{C}(9)-\mathrm{H}(9 \mathrm{~B})$ & 0.9900 \\
\hline $\mathrm{C}(00 \mathrm{R})-\mathrm{C}(13)$ & $1.526(4)$ \\
\hline $\mathrm{C}(00 \mathrm{R})-\mathrm{C}(17)$ & $1.529(5)$ \\
\hline $\mathrm{C}(00 \mathrm{R})-\mathrm{H}(00 \mathrm{~A})$ & 0.9900 \\
\hline $\mathrm{C}(00 \mathrm{R})-\mathrm{H}(00 \mathrm{~B})$ & 0.9900 \\
\hline$C(10)-H(10 A)$ & 0.9900 \\
\hline $\mathrm{C}(10)-\mathrm{H}(10 \mathrm{~B})$ & 0.9900 \\
\hline $\mathrm{C}(11)-\mathrm{C}(12)$ & $1.533(4)$ \\
\hline $\mathrm{C}(11)-\mathrm{C}(18)$ & $1.547(4)$ \\
\hline $\mathrm{C}(11)-\mathrm{C}(19)$ & $1.550(4)$ \\
\hline $\mathrm{C}(12)-\mathrm{C}(13)$ & $1.532(4)$ \\
\hline $\mathrm{C}(12)-\mathrm{H}(12 \mathrm{~A})$ & 0.9900 \\
\hline $\mathrm{C}(12)-\mathrm{H}(12 \mathrm{~B})$ & 0.9900 \\
\hline$C(13)-C(14)$ & $1.522(4)$ \\
\hline$C(14)-C(15)$ & $1.534(4)$ \\
\hline $\mathrm{C}(14)-\mathrm{H}(14 \mathrm{~A})$ & 0.9900 \\
\hline $\mathrm{C}(14)-\mathrm{H}(14 \mathrm{~B})$ & 0.9900 \\
\hline$C(15)-C(16)$ & $1.529(5)$ \\
\hline $\mathrm{C}(15)-\mathrm{C}(19)$ & $1.545(4)$ \\
\hline $\mathrm{C}(15)-\mathrm{H}(15)$ & 1.0000 \\
\hline$C(16)-C(17)$ & $1.536(5)$ \\
\hline $\mathrm{C}(16)-\mathrm{H}(16 \mathrm{~A})$ & 0.9900 \\
\hline $\mathrm{C}(16)-\mathrm{H}(16 \mathrm{~B})$ & 0.9900 \\
\hline$C(17)-C(18)$ & $1.539(5)$ \\
\hline $\mathrm{C}(17)-\mathrm{H}(17)$ & 1.0000 \\
\hline $\mathrm{C}(18)-\mathrm{H}(18 \mathrm{~A})$ & 0.9900 \\
\hline $\mathrm{C}(18)-\mathrm{H}(18 \mathrm{~B})$ & 0.9900 \\
\hline $\mathrm{C}(19)-\mathrm{H}(19 \mathrm{~A})$ & 0.9900 \\
\hline C(19)-H(19B) & 0.9900 \\
\hline $\mathrm{O}(2)-\mathrm{P}(1)-\mathrm{C}(1)$ & $116.61(13)$ \\
\hline $\mathrm{O}(2)-\mathrm{P}(1)-\mathrm{Cl}(1)$ & $112.48(10)$ \\
\hline $\mathrm{C}(1)-\mathrm{P}(1)-\mathrm{Cl}(1)$ & $107.29(10)$ \\
\hline $\mathrm{O}(2)-\mathrm{P}(1)-\mathrm{Cl}(2)$ & $111.26(10)$ \\
\hline $\mathrm{C}(1)-\mathrm{P}(1)-\mathrm{Cl}(2)$ & $106.10(9)$ \\
\hline $\mathrm{Cl}(1)-\mathrm{P}(1)-\mathrm{Cl}(2)$ & $101.89(5)$ \\
\hline
\end{tabular}




\begin{tabular}{|c|c|}
\hline $\mathrm{C}(3)-\mathrm{O}(1)-\mathrm{H}(1)$ & 109.5 \\
\hline $\mathrm{C}(9)-\mathrm{C}(1)-\mathrm{C}(8)$ & $109.7(2)$ \\
\hline $\mathrm{C}(9)-\mathrm{C}(1)-\mathrm{C}(2)$ & $109.8(2)$ \\
\hline $\mathrm{C}(8)-\mathrm{C}(1)-\mathrm{C}(2)$ & $109.5(2)$ \\
\hline C(9)-C(1)-P(1) & $113.60(19)$ \\
\hline $\mathrm{C}(8)-\mathrm{C}(1)-\mathrm{P}(1)$ & $107.89(18)$ \\
\hline $\mathrm{C}(2)-\mathrm{C}(1)-\mathrm{P}(1)$ & $106.23(18)$ \\
\hline $\mathrm{O}(4)-\mathrm{P}(2)-\mathrm{C}(11)$ & $116.64(13)$ \\
\hline $\mathrm{O}(4)-\mathrm{P}(2)-\mathrm{Cl}(3)$ & $111.44(10)$ \\
\hline $\mathrm{C}(11)-\mathrm{P}(2)-\mathrm{Cl}(3)$ & $106.74(10)$ \\
\hline $\mathrm{O}(4)-\mathrm{P}(2)-\mathrm{Cl}(4)$ & 111.29(9) \\
\hline $\mathrm{C}(11)-\mathrm{P}(2)-\mathrm{Cl}(4)$ & $106.86(10)$ \\
\hline $\mathrm{Cl}(3)-\mathrm{P}(2)-\mathrm{Cl}(4)$ & $102.84(5)$ \\
\hline $\mathrm{C}(3)-\mathrm{C}(2)-\mathrm{C}(1)$ & $109.1(2)$ \\
\hline $\mathrm{C}(3)-\mathrm{C}(2)-\mathrm{H}(2 \mathrm{~A})$ & 109.9 \\
\hline $\mathrm{C}(1)-\mathrm{C}(2)-\mathrm{H}(2 \mathrm{~A})$ & 109.9 \\
\hline $\mathrm{C}(3)-\mathrm{C}(2)-\mathrm{H}(2 \mathrm{~B})$ & 109.9 \\
\hline $\mathrm{C}(1)-\mathrm{C}(2)-\mathrm{H}(2 \mathrm{~B})$ & 109.9 \\
\hline $\mathrm{H}(2 \mathrm{~A})-\mathrm{C}(2)-\mathrm{H}(2 \mathrm{~B})$ & 108.3 \\
\hline $\mathrm{C}(13)-\mathrm{O}(3)-\mathrm{H}(3)$ & 109.5 \\
\hline $\mathrm{O}(1)-\mathrm{C}(3)-\mathrm{C}(10)$ & $106.6(2)$ \\
\hline $\mathrm{O}(1)-\mathrm{C}(3)-\mathrm{C}(4)$ & $111.9(2)$ \\
\hline $\mathrm{C}(10)-\mathrm{C}(3)-\mathrm{C}(4)$ & $110.4(2)$ \\
\hline $\mathrm{O}(1)-\mathrm{C}(3)-\mathrm{C}(2)$ & $110.1(2)$ \\
\hline$C(10)-C(3)-C(2)$ & $108.5(2)$ \\
\hline $\mathrm{C}(4)-\mathrm{C}(3)-\mathrm{C}(2)$ & $109.2(2)$ \\
\hline$C(3)-C(4)-C(5)$ & $109.7(2)$ \\
\hline $\mathrm{C}(3)-\mathrm{C}(4)-\mathrm{H}(4 \mathrm{~A})$ & 109.7 \\
\hline $\mathrm{C}(5)-\mathrm{C}(4)-\mathrm{H}(4 \mathrm{~A})$ & 109.7 \\
\hline $\mathrm{C}(3)-\mathrm{C}(4)-\mathrm{H}(4 \mathrm{~B})$ & 109.7 \\
\hline $\mathrm{C}(5)-\mathrm{C}(4)-\mathrm{H}(4 \mathrm{~B})$ & 109.7 \\
\hline $\mathrm{H}(4 \mathrm{~A})-\mathrm{C}(4)-\mathrm{H}(4 \mathrm{~B})$ & 108.2 \\
\hline$C(6)-C(5)-C(4)$ & $109.9(2)$ \\
\hline $\mathrm{C}(6)-\mathrm{C}(5)-\mathrm{C}(9)$ & $109.8(2)$ \\
\hline$C(4)-C(5)-C(9)$ & $109.4(2)$ \\
\hline $\mathrm{C}(6)-\mathrm{C}(5)-\mathrm{H}(5)$ & 109.2 \\
\hline $\mathrm{C}(4)-\mathrm{C}(5)-\mathrm{H}(5)$ & 109.2 \\
\hline $\mathrm{C}(9)-\mathrm{C}(5)-\mathrm{H}(5)$ & 109.2 \\
\hline$C(5)-C(6)-C(7)$ & $109.4(2)$ \\
\hline $\mathrm{C}(5)-\mathrm{C}(6)-\mathrm{H}(6 \mathrm{~A})$ & 109.8 \\
\hline $\mathrm{C}(7)-\mathrm{C}(6)-\mathrm{H}(6 \mathrm{~A})$ & 109.8 \\
\hline
\end{tabular}




\begin{tabular}{|c|c|}
\hline $\mathrm{C}(5)-\mathrm{C}(6)-\mathrm{H}(6 \mathrm{~B})$ & 109.8 \\
\hline $\mathrm{C}(7)-\mathrm{C}(6)-\mathrm{H}(6 \mathrm{~B})$ & 109.8 \\
\hline $\mathrm{H}(6 \mathrm{~A})-\mathrm{C}(6)-\mathrm{H}(6 \mathrm{~B})$ & 108.2 \\
\hline$C(10)-C(7)-C(8)$ & $109.8(2)$ \\
\hline$C(10)-C(7)-C(6)$ & $109.6(3)$ \\
\hline$C(8)-C(7)-C(6)$ & $109.3(2)$ \\
\hline $\mathrm{C}(10)-\mathrm{C}(7)-\mathrm{H}(7)$ & 109.4 \\
\hline $\mathrm{C}(8)-\mathrm{C}(7)-\mathrm{H}(7)$ & 109.4 \\
\hline $\mathrm{C}(6)-\mathrm{C}(7)-\mathrm{H}(7)$ & 109.4 \\
\hline $\mathrm{C}(7)-\mathrm{C}(8)-\mathrm{C}(1)$ & $108.4(2)$ \\
\hline $\mathrm{C}(7)-\mathrm{C}(8)-\mathrm{H}(8 \mathrm{~A})$ & 110.0 \\
\hline $\mathrm{C}(1)-\mathrm{C}(8)-\mathrm{H}(8 \mathrm{~A})$ & 110.0 \\
\hline $\mathrm{C}(7)-\mathrm{C}(8)-\mathrm{H}(8 \mathrm{~B})$ & 110.0 \\
\hline $\mathrm{C}(1)-\mathrm{C}(8)-\mathrm{H}(8 \mathrm{~B})$ & 110.0 \\
\hline $\mathrm{H}(8 \mathrm{~A})-\mathrm{C}(8)-\mathrm{H}(8 \mathrm{~B})$ & 108.4 \\
\hline$C(5)-C(9)-C(1)$ & $108.5(2)$ \\
\hline $\mathrm{C}(5)-\mathrm{C}(9)-\mathrm{H}(9 \mathrm{~A})$ & 110.0 \\
\hline $\mathrm{C}(1)-\mathrm{C}(9)-\mathrm{H}(9 \mathrm{~A})$ & 110.0 \\
\hline C(5)-C(9)-H(9B) & 110.0 \\
\hline $\mathrm{C}(1)-\mathrm{C}(9)-\mathrm{H}(9 \mathrm{~B})$ & 110.0 \\
\hline $\mathrm{H}(9 \mathrm{~A})-\mathrm{C}(9)-\mathrm{H}(9 \mathrm{~B})$ & 108.4 \\
\hline$C(13)-C(00 R)-C(17)$ & $110.0(3)$ \\
\hline $\mathrm{C}(13)-\mathrm{C}(00 \mathrm{R})-\mathrm{H}(00 \mathrm{~A})$ & 109.7 \\
\hline $\mathrm{C}(17)-\mathrm{C}(00 \mathrm{R})-\mathrm{H}(00 \mathrm{~A})$ & 109.7 \\
\hline $\mathrm{C}(13)-\mathrm{C}(00 \mathrm{R})-\mathrm{H}(00 \mathrm{~B})$ & 109.7 \\
\hline $\mathrm{C}(17)-\mathrm{C}(00 \mathrm{R})-\mathrm{H}(00 \mathrm{~B})$ & 109.7 \\
\hline $\mathrm{H}(00 \mathrm{~A})-\mathrm{C}(00 \mathrm{R})-\mathrm{H}(00 \mathrm{~B})$ & 108.2 \\
\hline$C(3)-C(10)-C(7)$ & $110.3(2)$ \\
\hline$C(3)-C(10)-H(10 A)$ & 109.6 \\
\hline $\mathrm{C}(7)-\mathrm{C}(10)-\mathrm{H}(10 \mathrm{~A})$ & 109.6 \\
\hline $\mathrm{C}(3)-\mathrm{C}(10)-\mathrm{H}(10 \mathrm{~B})$ & 109.6 \\
\hline $\mathrm{C}(7)-\mathrm{C}(10)-\mathrm{H}(10 \mathrm{~B})$ & 109.6 \\
\hline $\mathrm{H}(10 \mathrm{~A})-\mathrm{C}(10)-\mathrm{H}(10 \mathrm{~B})$ & 108.1 \\
\hline$C(12)-C(11)-C(18)$ & $109.9(2)$ \\
\hline$C(12)-C(11)-C(19)$ & $109.1(2)$ \\
\hline $\mathrm{C}(18)-\mathrm{C}(11)-\mathrm{C}(19)$ & $109.7(2)$ \\
\hline $\mathrm{C}(12)-\mathrm{C}(11)-\mathrm{P}(2)$ & $111.95(19)$ \\
\hline $\mathrm{C}(18)-\mathrm{C}(11)-\mathrm{P}(2)$ & $108.33(19)$ \\
\hline $\mathrm{C}(19)-\mathrm{C}(11)-\mathrm{P}(2)$ & $107.77(19)$ \\
\hline$C(13)-C(12)-C(11)$ & $109.4(2)$ \\
\hline $\mathrm{C}(13)-\mathrm{C}(12)-\mathrm{H}(12 \mathrm{~A})$ & 109.8 \\
\hline
\end{tabular}




\begin{tabular}{|c|c|}
\hline $\mathrm{C}(11)-\mathrm{C}(12)-\mathrm{H}(12 \mathrm{~A})$ & 109.8 \\
\hline $\mathrm{C}(13)-\mathrm{C}(12)-\mathrm{H}(12 \mathrm{~B})$ & 109.8 \\
\hline $\mathrm{C}(11)-\mathrm{C}(12)-\mathrm{H}(12 \mathrm{~B})$ & 109.8 \\
\hline $\mathrm{H}(12 \mathrm{~A})-\mathrm{C}(12)-\mathrm{H}(12 \mathrm{~B})$ & 108.2 \\
\hline $\mathrm{O}(3)-\mathrm{C}(13)-\mathrm{C}(14)$ & $107.3(2)$ \\
\hline $\mathrm{O}(3)-\mathrm{C}(13)-\mathrm{C}(00 \mathrm{R})$ & $111.0(2)$ \\
\hline $\mathrm{C}(14)-\mathrm{C}(13)-\mathrm{C}(00 \mathrm{R})$ & $110.5(3)$ \\
\hline $\mathrm{O}(3)-\mathrm{C}(13)-\mathrm{C}(12)$ & $109.6(2)$ \\
\hline $\mathrm{C}(14)-\mathrm{C}(13)-\mathrm{C}(12)$ & $109.6(2)$ \\
\hline $\mathrm{C}(00 \mathrm{R})-\mathrm{C}(13)-\mathrm{C}(12)$ & $108.9(2)$ \\
\hline $\mathrm{C}(13)-\mathrm{C}(14)-\mathrm{C}(15)$ & $109.0(2)$ \\
\hline $\mathrm{C}(13)-\mathrm{C}(14)-\mathrm{H}(14 \mathrm{~A})$ & 109.9 \\
\hline $\mathrm{C}(15)-\mathrm{C}(14)-\mathrm{H}(14 \mathrm{~A})$ & 109.9 \\
\hline $\mathrm{C}(13)-\mathrm{C}(14)-\mathrm{H}(14 \mathrm{~B})$ & 109.9 \\
\hline $\mathrm{C}(15)-\mathrm{C}(14)-\mathrm{H}(14 \mathrm{~B})$ & 109.9 \\
\hline $\mathrm{H}(14 \mathrm{~A})-\mathrm{C}(14)-\mathrm{H}(14 \mathrm{~B})$ & 108.3 \\
\hline$C(16)-C(15)-C(14)$ & $110.1(3)$ \\
\hline$C(16)-C(15)-C(19)$ & $110.1(3)$ \\
\hline C(14)-C(15)-C(19) & $109.4(2)$ \\
\hline $\mathrm{C}(16)-\mathrm{C}(15)-\mathrm{H}(15)$ & 109.1 \\
\hline $\mathrm{C}(14)-\mathrm{C}(15)-\mathrm{H}(15)$ & 109.1 \\
\hline $\mathrm{C}(19)-\mathrm{C}(15)-\mathrm{H}(15)$ & 109.1 \\
\hline$C(15)-C(16)-C(17)$ & $109.2(3)$ \\
\hline $\mathrm{C}(15)-\mathrm{C}(16)-\mathrm{H}(16 \mathrm{~A})$ & 109.8 \\
\hline $\mathrm{C}(17)-\mathrm{C}(16)-\mathrm{H}(16 \mathrm{~A})$ & 109.8 \\
\hline $\mathrm{C}(15)-\mathrm{C}(16)-\mathrm{H}(16 \mathrm{~B})$ & 109.8 \\
\hline $\mathrm{C}(17)-\mathrm{C}(16)-\mathrm{H}(16 \mathrm{~B})$ & 109.8 \\
\hline $\mathrm{H}(16 \mathrm{~A})-\mathrm{C}(16)-\mathrm{H}(16 \mathrm{~B})$ & 108.3 \\
\hline $\mathrm{C}(00 \mathrm{R})-\mathrm{C}(17)-\mathrm{C}(16)$ & $109.6(3)$ \\
\hline $\mathrm{C}(00 \mathrm{R})-\mathrm{C}(17)-\mathrm{C}(18)$ & $108.9(3)$ \\
\hline $\mathrm{C}(16)-\mathrm{C}(17)-\mathrm{C}(18)$ & $110.1(3)$ \\
\hline $\mathrm{C}(00 \mathrm{R})-\mathrm{C}(17)-\mathrm{H}(17)$ & 109.4 \\
\hline $\mathrm{C}(16)-\mathrm{C}(17)-\mathrm{H}(17)$ & 109.4 \\
\hline $\mathrm{C}(18)-\mathrm{C}(17)-\mathrm{H}(17)$ & 109.4 \\
\hline $\mathrm{C}(17)-\mathrm{C}(18)-\mathrm{C}(11)$ & $108.7(2)$ \\
\hline $\mathrm{C}(17)-\mathrm{C}(18)-\mathrm{H}(18 \mathrm{~A})$ & 110.0 \\
\hline $\mathrm{C}(11)-\mathrm{C}(18)-\mathrm{H}(18 \mathrm{~A})$ & 110.0 \\
\hline $\mathrm{C}(17)-\mathrm{C}(18)-\mathrm{H}(18 \mathrm{~B})$ & 110.0 \\
\hline $\mathrm{C}(11)-\mathrm{C}(18)-\mathrm{H}(18 \mathrm{~B})$ & 110.0 \\
\hline $\mathrm{H}(18 \mathrm{~A})-\mathrm{C}(18)-\mathrm{H}(18 \mathrm{~B})$ & 108.3 \\
\hline$C(15)-C(19)-C(11)$ & $108.4(2)$ \\
\hline
\end{tabular}




$\begin{array}{ll}\text { C(15)-C(19)-H(19A) } & 110.0 \\ \text { C(11)-C(19)-H(19A) } & 110.0 \\ \text { C(15)-C(19)-H(19B) } & 110.0 \\ \text { C(11)-C(19)-H(19B) } & 110.0 \\ \text { H(19A)-C(19)-H(19B) } & 108.4\end{array}$




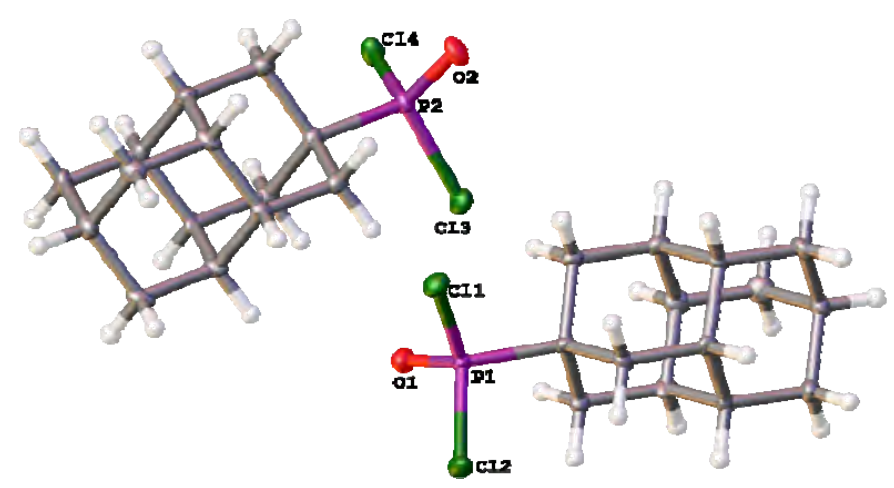

Ellipsoids are drawn at 50\% probability.

Experimental. A single colourless prism-shaped crystal (Compound 19) was selected and mounted on a Mo diffractometer. The crystal $(0.45 \times 0.35 \times 0.17)$ was kept at $T$ $=100 \mathrm{~K}$ during data collection. Using Olex2 (Dolomanov et al., 2009), the structure was solved with the ShelXS (Sheldrick, 2013) structure solution program, using the Direct Methods solution method. The model was refined with version 2013-3 of XL (Sheldrick, 2008) using Least Squares minimisation.

Crystal Data. $\mathrm{C}_{14} \mathrm{H}_{19} \mathrm{Cl}_{2} \mathrm{OP}, M_{r}=305.16$, monoclinic, $\mathrm{C} 2 / \mathrm{c}$ (No. 15), $\quad \mathrm{a}=21.691(3) \AA, \quad \mathrm{b}=16.4132(18) \AA, \quad \mathrm{c}=$ 15.261(2) $\AA, \beta=91.265(5)^{\circ}, \alpha=\gamma=90^{\circ}, V=5431.9(11) \AA^{3}$, $T=100 \mathrm{~K}, Z=16, Z^{\prime}=2, \mu\left(\mathrm{MoK}_{\alpha}\right)=0.581,53928$ reflections measured, 6298 unique $\left(R_{\text {int }}=0.0805\right)$ which were used in all calculations. The final $w R_{2}$ was 0.2193 (all data) and $R_{1}$ was $0.0672(\mathrm{I}>2(\mathrm{I})$ ).

\begin{tabular}{|c|c|}
\hline Compound & Compound 19 \\
\hline $\mathrm{N}^{\circ} \mathrm{CCDC}$ & 1054567 \\
\hline Formula & $\mathrm{C}_{14} \mathrm{H}_{19} \mathrm{Cl}_{2} \mathrm{OP}$ \\
\hline$D_{\text {calc }} / \mathrm{g} \mathrm{cm}^{-3}$ & 1.493 \\
\hline$\mu / \mathrm{mm}^{-1}$ & 0.581 \\
\hline Formula Weight & 305.16 \\
\hline Colour & colourless \\
\hline Shape & prism \\
\hline Max Size/mm & 0.45 \\
\hline Mid Size/mm & 0.35 \\
\hline Min Size/mm & 0.17 \\
\hline$T / \mathrm{K}$ & 100 \\
\hline Crystal System & monoclinic \\
\hline Space Group & $\mathrm{C} 2 / \mathrm{c}$ \\
\hline$a / \AA$ & 21.691(3) \\
\hline$b / \AA$ & $16.4132(18)$ \\
\hline$c / \AA ̊$ & $15.261(2)$ \\
\hline$\left.\alpha\right|^{\circ}$ & 90 \\
\hline$\beta 1^{\circ}$ & $91.265(5)$ \\
\hline$y 1^{\circ}$ & 90 \\
\hline $\mathrm{V} / \AA^{3}$ & $5431.9(11)$ \\
\hline Z & 16 \\
\hline$Z^{\prime}$ & 2 \\
\hline$\Theta_{\min } 1^{\circ}$ & 3.073 \\
\hline$\Theta_{\max } /^{\circ}$ & 27.796 \\
\hline Measured Refl. & 53928 \\
\hline Independent Refl. & 6298 \\
\hline Reflections Used & 4944 \\
\hline$R_{\text {int }}$ & 0.0805 \\
\hline Parameters & 326 \\
\hline Restraints & 0 \\
\hline Largest Peak & 0.805 \\
\hline Deepest Hole & -0.577 \\
\hline GooF & 1.063 \\
\hline$w R_{2}$ (all data) & 0.2193 \\
\hline$w R_{2}$ & 0.1989 \\
\hline$R_{1}$ (all data) & 0.0894 \\
\hline$R_{1}$ & 0.0672 \\
\hline
\end{tabular}


Experimental Extended. A colourless prism-shaped crystal with dimensions $0.45 \times 0.35 \times 0.17 \mathrm{~mm}^{3}$ was mounted on a mylar loop with grease. Data were collected using $\mathrm{MoK}_{\alpha}$ radiation (X-ray tube, $50 \mathrm{kV}$, $32 \mathrm{~mA}$ ). The total number of runs and images was based on the strategy calculation from the program APEX2. Cell parameters were retrieved using the SAINT (V8.34A, 2013) software and refined using SAINT (V8.34A, 2013) on 9846 reflections, 18 of the observed reflections. Data reduction was performed using the SAINT (V8.34A, 2013) software which corrects for Lorentz polarisation. The final completeness is 99.90 out to 27.796 in $\Theta$. The absorption coefficient $(\mu)$ of this material is 0.581 and the minimum and maximum transmissions are 0.6549 and 0.7456 . The structure was solved in the space group C2/c (\#15) by Direct Methods using the ShelXS-2013 (Sheldrick, 2015) structure solution program and refined by Least Squares using version 2013-3 of XL (Sheldrick, 2008) with a twin law (-1 0 - $-0.060-100012)$. All non-hydrogen atoms were refined anisotropically. Hydrogen atom positions were calculated geometrically and refined using the riding model. The value of $Z^{\prime}$ is 2 . This means that there are two independent molecules in the asymmetric unit.

Table S11: Fractional Atomic Coordinates $\left(\times 10^{4}\right)$ and Equivalent Isotropic Displacement Parameters $\left(\AA^{2} \times 10^{3}\right)$ for Compound 19. $U_{e q}$ is defined as $1 / 3$ of the trace of the orthogonalised $U_{i j}$.

\begin{tabular}{|c|c|c|c|c|}
\hline Atom & $\mathbf{x}$ & $\mathbf{y}$ & $\mathbf{z}$ & $U_{e q}$ \\
\hline$\overline{\mathrm{C} 11}$ & $4424.8(6)$ & $2931.4(7)$ & $3218.3(8)$ & $20.1(3)$ \\
\hline $\mathrm{Cl} 2$ & $3150.1(6)$ & $2229.1(8)$ & $3768.6(9)$ & $24.4(3)$ \\
\hline P1 & $3993.0(6)$ & $2520.7(7)$ & $4287.6(8)$ & $14.3(3)$ \\
\hline $\mathrm{O} 1$ & $4305.2(17)$ & $1818(2)$ & $4682(3)$ & $21.2(8)$ \\
\hline $\mathrm{C} 1$ & $3881(2)$ & $3393(3)$ & 4991(3) & $13.3(9)$ \\
\hline $\mathrm{C} 2$ & $3464(2)$ & $4054(3)$ & $4573(3)$ & $14.7(9)$ \\
\hline $\mathrm{C} 3$ & $3385(2)$ & $4748(3)$ & $5234(3)$ & $12.7(9)$ \\
\hline $\mathrm{C} 4$ & $4006(2)$ & $5131(3)$ & $5503(3)$ & $12.3(9)$ \\
\hline $\mathrm{C} 5$ & $4424(2)$ & $4459(3)$ & $5909(3)$ & $14.7(9)$ \\
\hline C6 & $4516(2)$ & $3766(3)$ & $5247(3)$ & $14.9(9)$ \\
\hline $\mathrm{C} 7$ & $4125(2)$ & 4139(3) & $6743(3)$ & $17.7(10)$ \\
\hline $\mathrm{C} 8$ & $3495(2)$ & $3753(3)$ & $6476(3)$ & $15.1(9)$ \\
\hline $\mathrm{C} 9$ & $3582(2)$ & $3050(3)$ & $5826(3)$ & $15.9(9)$ \\
\hline $\mathrm{C} 10$ & $3912(2)$ & $5816(3)$ & $6165(3)$ & $17.6(10)$ \\
\hline $\mathrm{C} 11$ & $3610(2)$ & $5482(3)$ & $6989(3)$ & $17.4(10)$ \\
\hline $\mathrm{C} 12$ & $2984(2)$ & $5103(3)$ & $6729(3)$ & $18.6(10)$ \\
\hline $\mathrm{C} 13$ & $3079(2)$ & $4419(3)$ & $6063(3)$ & $13.3(9)$ \\
\hline $\mathrm{C} 14$ & $4031(3)$ & $4823(3)$ & $7406(3)$ & $19.6(10)$ \\
\hline $\mathrm{Cl} 3$ & $5659.3(6)$ & $2388.4(8)$ & $6807.9(8)$ & $21.3(3)$ \\
\hline $\mathrm{Cl} 4$ & $7090.6(6)$ & $2084.8(7)$ & $6964.1(8)$ & $20.1(3)$ \\
\hline $\mathrm{P} 2$ & $6428.7(6)$ & $2465.5(7)$ & $6089.5(8)$ & $13.6(3)$ \\
\hline $\mathrm{O} 2$ & $6541.2(18)$ & $3298(2)$ & $5790(2)$ & $21.7(8)$ \\
\hline $\mathrm{C} 15$ & $6350(2)$ & $1670(3)$ & $5272(3)$ & $12.4(9)$ \\
\hline $\mathrm{C} 16$ & $6201(2)$ & $835(3)$ & $5680(3)$ & $11.9(8)$ \\
\hline $\mathrm{C} 17$ & $6125(2)$ & 204(3) & $4948(3)$ & $12.2(9)$ \\
\hline C18 & $5592(2)$ & 451(3) & $4311(3)$ & 13.2(9) \\
\hline C19 & $5743(2)$ & $1282(3)$ & $3909(3)$ & 14.1(9) \\
\hline $\mathrm{C} 20$ & $5810(2)$ & $1924(3)$ & $4630(3)$ & $14.6(9)$ \\
\hline $\mathrm{C} 21$ & $6341(2)$ & $1209(3)$ & $3390(3)$ & $15.4(9)$ \\
\hline $\mathrm{C} 22$ & $6870(2)$ & $968(3)$ & $4038(3)$ & $14.7(9)$ \\
\hline $\mathrm{C} 23$ & $6951(2)$ & $1621(3)$ & $4763(3)$ & $15.3(9)$ \\
\hline $\mathrm{C} 24$ & $5515(2)$ & $-205(3)$ & $3595(3)$ & $16.1(10)$ \\
\hline $\mathrm{C} 25$ & $6111(2)$ & $-266(3)$ & $3075(3)$ & $17.7(10)$ \\
\hline $\mathrm{C} 26$ & $6647(2)$ & $-517(3)$ & $3712(3)$ & $17.6(10)$ \\
\hline $\mathrm{C} 27$ & $6721(2)$ & 131(3) & $4433(3)$ & $13.9(9)$ \\
\hline $\mathrm{C} 28$ & $6257(2)$ & $561(3)$ & $2669(3)$ & $18(1)$ \\
\hline
\end{tabular}


Table S12: Anisotropic Displacement Parameters $\left(\times 10^{4}\right)$ Compound 19. The anisotropic displacement factor exponent takes the form: $-2 \pi^{2}\left[h^{2} a^{*^{2}} \times U_{11}+\ldots+2 h k a^{*} \times b^{*} \times U_{12}\right]$

\begin{tabular}{|c|c|c|c|c|c|c|}
\hline Atom & $U_{11}$ & $U_{22}$ & $U_{33}$ & $U_{23}$ & $U_{13}$ & $U_{12}$ \\
\hline $\mathrm{Cl1}$ & $18.0(6)$ & $20.9(6)$ & $21.8(6)$ & $0.7(4)$ & $7.1(4)$ & $1.1(4)$ \\
\hline $\mathrm{Cl} 2$ & $13.8(6)$ & $25.1(6)$ & $34.4(7)$ & $-12.8(5)$ & $1.7(5)$ & $-3.7(5)$ \\
\hline P1 & $10.8(6)$ & $11.3(6)$ & $21.0(6)$ & $-1.1(4)$ & $2.9(5)$ & $-0.5(4)$ \\
\hline $\mathrm{O} 1$ & $18.9(18)$ & $13.1(17)$ & $32(2)$ & $2.3(15)$ & $5.3(15)$ & $1.9(14)$ \\
\hline $\mathrm{C} 1$ & $8(2)$ & $14(2)$ & $18(2)$ & $1.1(17)$ & $1.3(17)$ & $-0.6(16)$ \\
\hline $\mathrm{C} 2$ & $16(2)$ & $9(2)$ & 19(2) & $1.8(17)$ & $-1.0(18)$ & $-0.6(17)$ \\
\hline C3 & $10(2)$ & 11(2) & $17(2)$ & $1.4(16)$ & $-3.7(17)$ & $2.3(16)$ \\
\hline $\mathrm{C} 4$ & $11(2)$ & $8(2)$ & $18(2)$ & $3.3(16)$ & $1.9(17)$ & $-1.3(16)$ \\
\hline $\mathrm{C} 5$ & 11(2) & $15(2)$ & $18(2)$ & $3.4(18)$ & $0.2(17)$ & $1.2(17)$ \\
\hline C6 & $9(2)$ & $15(2)$ & 21(2) & $3.9(18)$ & $1.2(17)$ & $-2.1(17)$ \\
\hline C7 & $19(2)$ & $16(2)$ & $18(2)$ & $4.6(18)$ & $-1.9(18)$ & $0.7(19)$ \\
\hline $\mathrm{C} 8$ & $18(2)$ & $10(2)$ & $18(2)$ & $6.1(17)$ & $7.7(18)$ & $0.2(17)$ \\
\hline C9 & $15(2)$ & 11(2) & $22(2)$ & $4.2(18)$ & $5.9(18)$ & $0.0(17)$ \\
\hline C10 & $21(2)$ & $12(2)$ & $20(2)$ & $-0.6(18)$ & $0.5(19)$ & $-4.5(18)$ \\
\hline C11 & $20(2)$ & $12(2)$ & $20(2)$ & $-1.0(18)$ & $2.2(19)$ & $-2.1(18)$ \\
\hline $\mathrm{C} 12$ & $17(2)$ & $13(2)$ & $26(3)$ & $-3.0(19)$ & $4(2)$ & $1.8(18)$ \\
\hline $\mathrm{C} 13$ & $10(2)$ & $9(2)$ & $22(2)$ & $1.4(17)$ & $0.0(17)$ & $1.0(16)$ \\
\hline $\mathrm{C} 14$ & $23(3)$ & $20(3)$ & $16(2)$ & $2.6(19)$ & $-2.9(19)$ & $-1(2)$ \\
\hline $\mathrm{Cl} 3$ & $20.4(6)$ & $22.0(6)$ & $21.7(6)$ & $-6.2(5)$ & $4.8(5)$ & $2.5(5)$ \\
\hline $\mathrm{Cl} 4$ & $21.9(6)$ & $17.2(6)$ & $20.8(6)$ & $-4.4(4)$ & $-6.7(5)$ & $2.8(4)$ \\
\hline P2 & $15.8(6)$ & $10.2(6)$ & $14.8(6)$ & $-1.7(4)$ & $-0.8(4)$ & $0.2(4)$ \\
\hline $\mathrm{O} 2$ & $30(2)$ & 10.1(17) & $25.2(18)$ & $0.7(14)$ & $-2.4(15)$ & $-1.2(14)$ \\
\hline C15 & $14(2)$ & $10(2)$ & $13(2)$ & $0.7(16)$ & $1.2(17)$ & $1.0(16)$ \\
\hline C16 & $13(2)$ & $12(2)$ & $10.0(19)$ & $3.7(16)$ & $1.1(16)$ & $0.4(17)$ \\
\hline C17 & $16(2)$ & $6(2)$ & $15(2)$ & $2.6(16)$ & $4.1(17)$ & $0.5(16)$ \\
\hline $\mathrm{C} 18$ & $14(2)$ & $14(2)$ & $12(2)$ & $2.0(17)$ & $0.5(17)$ & $-3.9(17)$ \\
\hline C19 & $13(2)$ & $12(2)$ & $17(2)$ & $3.1(17)$ & $-1.3(17)$ & $-1.5(17)$ \\
\hline $\mathrm{C} 20$ & $13(2)$ & $14(2)$ & $17(2)$ & $3.5(17)$ & $1.1(17)$ & $1.1(17)$ \\
\hline $\mathrm{C} 21$ & $19(2)$ & $12(2)$ & $15(2)$ & $3.0(17)$ & $-2.6(18)$ & $-5.5(18)$ \\
\hline $\mathrm{C} 22$ & $10(2)$ & $16(2)$ & $18(2)$ & $0.3(18)$ & $2.2(17)$ & $-0.1(17)$ \\
\hline $\mathrm{C} 23$ & $13(2)$ & $16(2)$ & $17(2)$ & $-0.9(18)$ & $1.0(17)$ & $-2.7(18)$ \\
\hline $\mathrm{C} 24$ & $19(2)$ & $15(2)$ & $14(2)$ & $4.1(17)$ & $-1.2(18)$ & $-6.7(18)$ \\
\hline $\mathrm{C} 25$ & $23(3)$ & $15(2)$ & $16(2)$ & $-3.3(18)$ & $1.2(19)$ & $-2.1(18)$ \\
\hline $\mathrm{C} 26$ & $20(2)$ & $12(2)$ & 21(2) & $-4.9(18)$ & $-1.5(19)$ & $2.3(18)$ \\
\hline C27 & $14(2)$ & $15(2)$ & $13(2)$ & $-4.8(17)$ & $-1.7(17)$ & $1.5(17)$ \\
\hline $\mathrm{C} 28$ & $22(3)$ & $18(2)$ & $14(2)$ & $1.8(18)$ & $1.2(18)$ & $-4.1(19)$ \\
\hline
\end{tabular}

Table S13: Bond Lengths in $\AA$ for Compound 19.

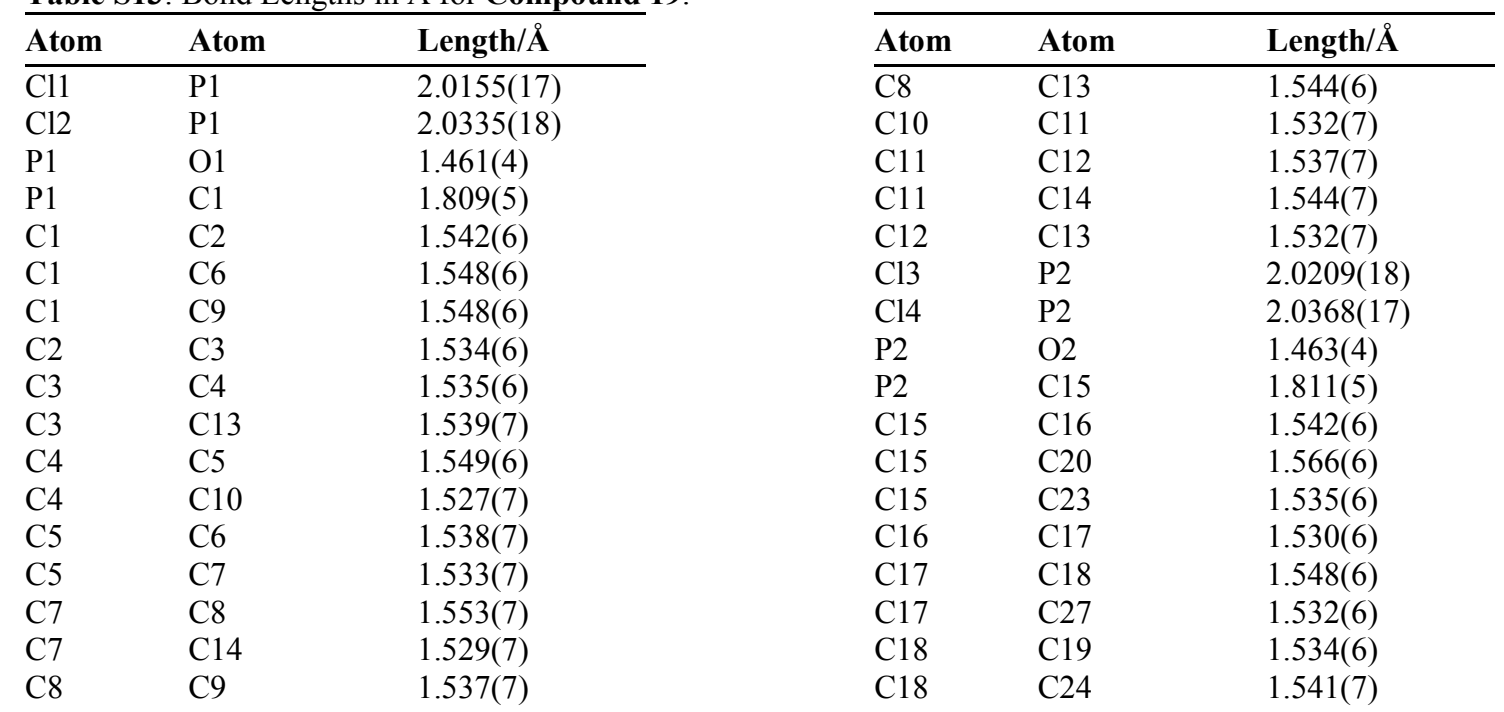




\begin{tabular}{|c|c|c|}
\hline Atom & Atom & Length/Å \\
\hline C19 & $\mathrm{C} 20$ & $1.529(7)$ \\
\hline C19 & $\mathrm{C} 21$ & $1.539(7)$ \\
\hline $\mathrm{C} 21$ & $\mathrm{C} 22$ & $1.549(6)$ \\
\hline $\mathrm{C} 21$ & $\mathrm{C} 28$ & $1.538(7)$ \\
\hline $\mathrm{C} 22$ & $\mathrm{C} 23$ & $1.549(7)$ \\
\hline
\end{tabular}

\begin{tabular}{lll}
\hline Atom & Atom & Length/A \\
\hline $\mathrm{C} 22$ & $\mathrm{C} 27$ & $1.537(7)$ \\
$\mathrm{C} 24$ & $\mathrm{C} 25$ & $1.536(7)$ \\
$\mathrm{C} 25$ & $\mathrm{C} 26$ & $1.555(7)$ \\
$\mathrm{C} 25$ & $\mathrm{C} 28$ & $1.528(7)$ \\
$\mathrm{C} 26$ & $\mathrm{C} 27$ & $1.537(6)$
\end{tabular}

Table S14: Bond Angles in ${ }^{\circ}$ for Compound 19.

\begin{tabular}{|c|c|c|c|}
\hline Atom & Atom & Atom & Angle/ ${ }^{\circ}$ \\
\hline$\overline{\mathrm{Cl1}}$ & P1 & $\mathrm{Cl} 2$ & $101.15(8)$ \\
\hline $\mathrm{O} 1$ & $\mathrm{P} 1$ & $\mathrm{Cl1}$ & $112.24(16)$ \\
\hline $\mathrm{O} 1$ & $\mathrm{P} 1$ & $\mathrm{Cl} 2$ & $112.30(17)$ \\
\hline $\mathrm{O} 1$ & $\mathrm{P} 1$ & $\mathrm{C} 1$ & $116.6(2)$ \\
\hline $\mathrm{C} 1$ & $\mathrm{P} 1$ & $\mathrm{Cl1}$ & $106.63(16)$ \\
\hline $\mathrm{C} 1$ & $\mathrm{P} 1$ & $\mathrm{Cl} 2$ & $106.64(16)$ \\
\hline $\mathrm{C} 2$ & $\mathrm{C} 1$ & P1 & $113.4(3)$ \\
\hline $\mathrm{C} 2$ & $\mathrm{C} 1$ & C6 & $109.7(4)$ \\
\hline $\mathrm{C} 2$ & $\mathrm{C} 1$ & C9 & $110.1(4)$ \\
\hline C6 & $\mathrm{C} 1$ & $\mathrm{P} 1$ & $109.4(3)$ \\
\hline C6 & $\mathrm{C} 1$ & C9 & $108.8(4)$ \\
\hline $\mathrm{C} 9$ & $\mathrm{C} 1$ & P1 & $105.3(3)$ \\
\hline $\mathrm{C} 3$ & $\mathrm{C} 2$ & $\mathrm{C} 1$ & $108.9(4)$ \\
\hline $\mathrm{C} 2$ & $\mathrm{C} 3$ & $\mathrm{C} 4$ & $111.7(4)$ \\
\hline $\mathrm{C} 2$ & $\mathrm{C} 3$ & $\mathrm{C} 13$ & $109.7(4)$ \\
\hline $\mathrm{C} 4$ & $\mathrm{C} 3$ & $\mathrm{C} 13$ & $108.4(4)$ \\
\hline $\mathrm{C} 3$ & $\mathrm{C} 4$ & $\mathrm{C} 5$ & $108.5(4)$ \\
\hline $\mathrm{C} 10$ & $\mathrm{C} 4$ & $\mathrm{C} 3$ & $110.5(4)$ \\
\hline $\mathrm{C} 10$ & $\mathrm{C} 4$ & $\mathrm{C} 5$ & $110.1(4)$ \\
\hline C6 & $\mathrm{C} 5$ & $\mathrm{C} 4$ & $110.3(4)$ \\
\hline $\mathrm{C} 7$ & $\mathrm{C} 5$ & $\mathrm{C} 4$ & $108.8(4)$ \\
\hline $\mathrm{C} 7$ & $\mathrm{C} 5$ & C6 & $110.8(4)$ \\
\hline $\mathrm{C} 5$ & C6 & $\mathrm{C} 1$ & $109.3(4)$ \\
\hline $\mathrm{C} 5$ & $\mathrm{C} 7$ & $\mathrm{C} 8$ & $108.0(4)$ \\
\hline $\mathrm{C} 14$ & $\mathrm{C} 7$ & $\mathrm{C} 5$ & $111.3(4)$ \\
\hline $\mathrm{C} 14$ & $\mathrm{C} 7$ & $\mathrm{C} 8$ & $110.1(4)$ \\
\hline C9 & $\mathrm{C} 8$ & $\mathrm{C} 7$ & $110.8(4)$ \\
\hline C9 & $\mathrm{C} 8$ & $\mathrm{C} 13$ & $110.3(4)$ \\
\hline C13 & $\mathrm{C} 8$ & $\mathrm{C} 7$ & $108.7(4)$ \\
\hline $\mathrm{C} 8$ & C9 & $\mathrm{C} 1$ & $108.5(4)$ \\
\hline $\mathrm{C} 4$ & $\mathrm{C} 10$ & $\mathrm{C} 11$ & $110.2(4)$ \\
\hline $\mathrm{C} 10$ & $\mathrm{C} 11$ & $\mathrm{C} 12$ & $109.0(4)$ \\
\hline $\mathrm{C} 10$ & $\mathrm{C} 11$ & $\mathrm{C} 14$ & $109.2(4)$ \\
\hline C12 & $\mathrm{C} 11$ & $\mathrm{C} 14$ & $109.5(4)$ \\
\hline C13 & $\mathrm{C} 12$ & $\mathrm{C} 11$ & 109.7(4) \\
\hline $\mathrm{C} 3$ & C13 & $\mathrm{C} 8$ & $109.0(4)$ \\
\hline $\mathrm{C} 12$ & $\mathrm{C} 13$ & $\mathrm{C} 3$ & $110.8(4)$ \\
\hline $\mathrm{C} 12$ & $\mathrm{C} 13$ & $\mathrm{C} 8$ & $109.4(4)$ \\
\hline $\mathrm{C} 7$ & $\mathrm{C} 14$ & $\mathrm{C} 11$ & $109.1(4)$ \\
\hline $\mathrm{Cl} 3$ & P2 & $\mathrm{Cl} 4$ & $101.76(8)$ \\
\hline $\mathrm{O} 2$ & $\mathrm{P} 2$ & $\mathrm{Cl} 3$ & $111.94(17)$ \\
\hline $\mathrm{O} 2$ & $\mathrm{P} 2$ & $\mathrm{Cl} 4$ & $111.81(17)$ \\
\hline $\mathrm{O} 2$ & $\mathrm{P} 2$ & $\mathrm{C} 15$ & $118.1(2)$ \\
\hline $\mathrm{C} 15$ & $\mathrm{P} 2$ & $\mathrm{Cl} 3$ & $105.17(16)$ \\
\hline $\mathrm{C} 15$ & $\mathrm{P} 2$ & $\mathrm{Cl} 4$ & $106.55(16)$ \\
\hline C16 & C15 & $\mathrm{P} 2$ & $112.3(3)$ \\
\hline C16 & $\mathrm{C} 15$ & $\mathrm{C} 20$ & $109.2(4)$ \\
\hline $\mathrm{C} 20$ & $\mathrm{C} 15$ & $\mathrm{P} 2$ & $107.3(3)$ \\
\hline $\mathrm{C} 23$ & C15 & $\mathrm{P} 2$ & $108.5(3)$ \\
\hline $\mathrm{C} 23$ & $\mathrm{C} 15$ & $\mathrm{C} 16$ & $110.2(4)$ \\
\hline $\mathrm{C} 23$ & $\mathrm{C} 15$ & $\mathrm{C} 20$ & 109.3(4) \\
\hline $\mathrm{C} 17$ & $\mathrm{C} 16$ & $\mathrm{C} 15$ & $109.1(3)$ \\
\hline
\end{tabular}

\begin{tabular}{llll}
\hline Atom & Atom & Atom & Angle ${ }^{\circ}$ \\
\hline C16 & C17 & C18 & $110.3(4)$ \\
C16 & C17 & C27 & $110.4(4)$ \\
C27 & C17 & C18 & $109.0(4)$ \\
C19 & C18 & C17 & $108.7(4)$ \\
C19 & C18 & C24 & $111.0(4)$ \\
C24 & C18 & C17 & $109.2(4)$ \\
C18 & C19 & C21 & $109.0(4)$ \\
C20 & C19 & C18 & $110.0(4)$ \\
C20 & C19 & C21 & $110.9(4)$ \\
C19 & C20 & C15 & $108.9(4)$ \\
C19 & C21 & C22 & $108.2(4)$ \\
C28 & C21 & C19 & $109.5(4)$ \\
C28 & C21 & C22 & $110.7(4)$ \\
C21 & C22 & C23 & $110.5(4)$ \\
C27 & C22 & C21 & $108.6(4)$ \\
C27 & C22 & C23 & $111.0(4)$ \\
C15 & C23 & C22 & $108.3(4)$ \\
C25 & C24 & C18 & $109.4(4)$ \\
C24 & C25 & C26 & $108.8(4)$ \\
C28 & C25 & C24 & $109.7(4)$ \\
C28 & C25 & C26 & $109.3(4)$ \\
C27 & C26 & C25 & $109.2(4)$ \\
C17 & C27 & C22 & $108.6(4)$ \\
C17 & C27 & C26 & $110.2(4)$ \\
C22 & C27 & C26 & $110.9(4)$ \\
C25 & C28 & C21 & $110.2(4)$ \\
& & &
\end{tabular}


Table S15: Hydrogen Fractional Atomic Coordinates $\left(\times 10^{4}\right)$ and Equivalent Isotropic Displacement Parameters $\left(\AA^{2} \times 10^{3}\right)$ for Compound 19. $U_{e q}$ is defined as $1 / 3$ of the trace of the orthogonalised $U_{i j}$.

\begin{tabular}{|c|c|c|c|c|}
\hline Atom & & & & $U_{e q}$ \\
\hline$\overline{\mathrm{H} 2 \mathrm{~A}}$ & 3653 & 4266 & 4034 & 18 \\
\hline $\mathrm{H} 2 \mathrm{~B}$ & 3057 & 3819 & 4412 & 18 \\
\hline H3 & 3114 & 5177 & 4964 & 15 \\
\hline H4 & 4206 & 5358 & 4972 & 15 \\
\hline H5 & 4835 & 4701 & 6068 & 18 \\
\hline H6A & 4786 & 3341 & 5510 & 18 \\
\hline H6B & 4716 & 3980 & 4718 & 18 \\
\hline H7 & 4396 & 3711 & 7013 & 21 \\
\hline H8 & 3295 & 3537 & 7013 & 18 \\
\hline H9A & 3179 & 2800 & 5673 & 19 \\
\hline H9B & 3851 & 2625 & 6093 & 19 \\
\hline H10A & 3648 & 6244 & 5899 & 21 \\
\hline $\mathrm{H} 10 \mathrm{~B}$ & 4316 & 6063 & 6326 & 21 \\
\hline H11 & 3548 & 5934 & 7417 & 21 \\
\hline $\mathrm{H} 12 \mathrm{~A}$ & 2785 & 4882 & 7256 & 22 \\
\hline $\mathrm{H} 12 \mathrm{~B}$ & 2711 & 5527 & 6471 & 22 \\
\hline H13 & 2669 & 4176 & 5897 & 16 \\
\hline H14A & 4434 & 5065 & 7579 & 24 \\
\hline H14B & 3839 & 4602 & 7939 & 24 \\
\hline H16A & 6539 & 670 & 6088 & 14 \\
\hline H16B & 5816 & 872 & 6014 & 14 \\
\hline H17 & 6028 & -337 & 5213 & 15 \\
\hline H18 & 5201 & 495 & 4644 & 16 \\
\hline H19 & 5399 & 1445 & 3498 & 17 \\
\hline $\mathrm{H} 20 \mathrm{~A}$ & 5421 & 1967 & 4954 & 17 \\
\hline $\mathrm{H} 20 \mathrm{~B}$ & 5899 & 2462 & 4368 & 17 \\
\hline $\mathrm{H} 21$ & 6438 & 1746 & 3116 & 18 \\
\hline $\mathrm{H} 22$ & 7262 & 925 & 3708 & 18 \\
\hline $\mathrm{H} 23 \mathrm{~A}$ & 7298 & 1471 & 5165 & 18 \\
\hline $\mathrm{H} 23 \mathrm{~B}$ & 7044 & 2156 & 4498 & 18 \\
\hline $\mathrm{H} 24 \mathrm{~A}$ & 5424 & -738 & 3868 & 19 \\
\hline $\mathrm{H} 24 \mathrm{~B}$ & 5166 & -60 & 3196 & 19 \\
\hline $\mathrm{H} 25$ & 6061 & -684 & 2603 & 21 \\
\hline $\mathrm{H} 26 \mathrm{~A}$ & 6557 & -1053 & 3978 & 21 \\
\hline $\mathrm{H} 26 \mathrm{~B}$ & 7035 & -565 & 3386 & 21 \\
\hline $\mathrm{H} 27$ & 7065 & -33 & 4844 & 17 \\
\hline $\mathrm{H} 28 \mathrm{~A}$ & 5917 & 724 & 2263 & 22 \\
\hline $\mathrm{H} 28 \mathrm{~B}$ & 6639 & 520 & 2329 & 22 \\
\hline
\end{tabular}




\section{(21) (4-Nitroxydiamant-1-yl)phosphonic dichloride}

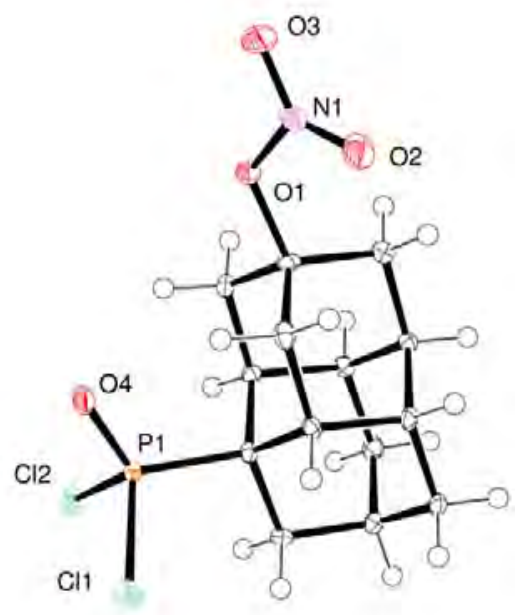

Ellipsoids are drawn at $50 \%$ probability.

Table S16. Crystal data and structure refinement for Compound 21.

CCDC No.

Empirical formula

Formula weight

Temperature

Wavelength

Crystal system

Space group

Unit cell dimensions

Volume

Z

Density (calculated)

Absorption coefficient

$\mathrm{F}(000)$

Crystal size

Theta range for data collection

Index ranges

Reflections collected

Independent reflections

Completeness to theta $=25.026^{\circ}$

Absorption correction

Max. and min. transmission

Refinement method
1053888

C14 H18 Cl2 N O4 P

366.16

100(2) K

$0.71073 \AA$

Orthorhombic

$\mathrm{Pna}_{1}$

$\mathrm{a}=21.9437(9) \AA \quad \alpha=90^{\circ}$.

$\mathrm{b}=10.5867(4) \AA \quad \beta=90^{\circ}$.

$\mathrm{c}=6.4754(3) \AA \quad \gamma=90^{\circ}$.

1504.31(11) $\AA^{3}$

4

$1.617 \mathrm{Mg} / \mathrm{m}^{3}$

$0.555 \mathrm{~mm}^{-1}$

760

$0.415 \times 0.240 \times 0.136 \mathrm{~mm}^{3}$

2.136 to $25.026^{\circ}$.

$-26<=\mathrm{h}<=26,-12<=\mathrm{k}<=12,-7<=\mathrm{l}<=7$

15603

$2651[\mathrm{R}($ int $)=0.0333]$

$100.0 \%$

Multiscan

0.7452 and 0.6631

Full-matrix least-squares on $\mathrm{F}^{2}$ 
Data / restraints / parameters

Goodness-of-fit on $\mathrm{F}^{2}$

Final $\mathrm{R}$ indices $[\mathrm{I}>2 \operatorname{sigma}(\mathrm{I})]$

$\mathrm{R}$ indices (all data)

Absolute structure parameter

Extinction coefficient

Largest diff. peak and hole
$2651 / 1 / 199$

1.087

$\mathrm{R} 1=0.0205, \mathrm{wR} 2=0.0512$

$\mathrm{R} 1=0.0214, \mathrm{wR} 2=0.0516$

$0.06(2)$

$\mathrm{n} / \mathrm{a}$

0.236 and -0.189 e. $\AA^{-3}$ 
Table S17. Bond lengths $[\AA]$ and angles $\left[{ }^{\circ}\right]$ for Compound 21.

\begin{tabular}{|c|c|}
\hline $\mathrm{Cl}(1)-\mathrm{P}(1)$ & $2.0156(9)$ \\
\hline $\mathrm{N}(1)-\mathrm{O}(2)$ & $1.205(3)$ \\
\hline $\mathrm{N}(1)-\mathrm{O}(3)$ & $1.213(3)$ \\
\hline $\mathrm{N}(1)-\mathrm{O}(1)$ & $1.399(3)$ \\
\hline $\mathrm{P}(1)-\mathrm{O}(4)$ & $1.4640(16)$ \\
\hline $\mathrm{P}(1)-\mathrm{C}(8)$ & $1.834(2)$ \\
\hline $\mathrm{P}(1)-\mathrm{Cl}(2)$ & $2.0194(9)$ \\
\hline $\mathrm{O}(1)-\mathrm{C}(13)$ & $1.490(3)$ \\
\hline $\mathrm{C}(1)-\mathrm{C}(5)$ & $1.527(3)$ \\
\hline $\mathrm{C}(1)-\mathrm{C}(2)$ & $1.532(4)$ \\
\hline $\mathrm{C}(1)-\mathrm{H}(1 \mathrm{~A})$ & 0.9900 \\
\hline $\mathrm{C}(1)-\mathrm{H}(1 \mathrm{~B})$ & 0.9900 \\
\hline$C(2)-C(4)$ & $1.522(3)$ \\
\hline$C(2)-C(3)$ & $1.525(3)$ \\
\hline $\mathrm{C}(2)-\mathrm{H}(2)$ & 1.0000 \\
\hline $\mathrm{C}(3)-\mathrm{C}(8)$ & $1.551(3)$ \\
\hline $\mathrm{C}(3)-\mathrm{H}(3 \mathrm{~A})$ & 0.9900 \\
\hline $\mathrm{C}(3)-\mathrm{H}(3 \mathrm{~B})$ & 0.9900 \\
\hline$C(4)-C(6)$ & $1.527(3)$ \\
\hline $\mathrm{C}(4)-\mathrm{H}(4 \mathrm{~A})$ & 0.9900 \\
\hline $\mathrm{C}(4)-\mathrm{H}(4 \mathrm{~B})$ & 0.9900 \\
\hline$C(5)-C(10)$ & $1.541(3)$ \\
\hline$C(5)-C(9)$ & $1.554(3)$ \\
\hline $\mathrm{C}(5)-\mathrm{H}(5)$ & 1.0000 \\
\hline$C(6)-C(10)$ & $1.535(3)$ \\
\hline$C(6)-C(7)$ & $1.548(3)$ \\
\hline $\mathrm{C}(6)-\mathrm{H}(6)$ & 1.0000 \\
\hline$C(7)-C(14)$ & $1.537(3)$ \\
\hline$C(7)-C(8)$ & $1.556(3)$ \\
\hline $\mathrm{C}(7)-\mathrm{H}(7)$ & 1.0000 \\
\hline $\mathrm{C}(8)-\mathrm{C}(9)$ & $1.564(3)$ \\
\hline $\mathrm{C}(9)-\mathrm{C}(12)$ & $1.540(3)$ \\
\hline $\mathrm{C}(9)-\mathrm{H}(9)$ & 1.0000 \\
\hline $\mathrm{C}(10)-\mathrm{C}(11)$ & $1.539(3)$ \\
\hline $\mathrm{C}(10)-\mathrm{H}(10)$ & 1.0000 \\
\hline$C(11)-C(13)$ & $1.522(3)$ \\
\hline $\mathrm{C}(11)-\mathrm{H}(11 \mathrm{~A})$ & 0.9900 \\
\hline $\mathrm{C}(11)-\mathrm{H}(11 \mathrm{~B})$ & 0.9900 \\
\hline
\end{tabular}




\begin{tabular}{|c|c|}
\hline$C(12)-C(13)$ & $1.529(3)$ \\
\hline $\mathrm{C}(12)-\mathrm{H}(12 \mathrm{~A})$ & 0.9900 \\
\hline $\mathrm{C}(12)-\mathrm{H}(12 \mathrm{~B})$ & 0.9900 \\
\hline $\mathrm{C}(13)-\mathrm{C}(14)$ & $1.530(3)$ \\
\hline C(14)-H(14A) & 0.9900 \\
\hline C(14)-H(14B) & 0.9900 \\
\hline $\mathrm{O}(2)-\mathrm{N}(1)-\mathrm{O}(3)$ & $128.7(2)$ \\
\hline $\mathrm{O}(2)-\mathrm{N}(1)-\mathrm{O}(1)$ & $119.57(19)$ \\
\hline $\mathrm{O}(3)-\mathrm{N}(1)-\mathrm{O}(1)$ & $111.7(2)$ \\
\hline $\mathrm{O}(4)-\mathrm{P}(1)-\mathrm{C}(8)$ & $119.19(10)$ \\
\hline $\mathrm{O}(4)-\mathrm{P}(1)-\mathrm{Cl}(1)$ & $110.86(8)$ \\
\hline $\mathrm{C}(8)-\mathrm{P}(1)-\mathrm{Cl}(1)$ & $106.97(8)$ \\
\hline $\mathrm{O}(4)-\mathrm{P}(1)-\mathrm{Cl}(2)$ & $111.96(9)$ \\
\hline $\mathrm{C}(8)-\mathrm{P}(1)-\mathrm{Cl}(2)$ & $104.01(8)$ \\
\hline $\mathrm{Cl}(1)-\mathrm{P}(1)-\mathrm{Cl}(2)$ & $102.35(4)$ \\
\hline $\mathrm{N}(1)-\mathrm{O}(1)-\mathrm{C}(13)$ & $118.36(18)$ \\
\hline $\mathrm{C}(5)-\mathrm{C}(1)-\mathrm{C}(2)$ & 109.12(19) \\
\hline $\mathrm{C}(5)-\mathrm{C}(1)-\mathrm{H}(1 \mathrm{~A})$ & 109.9 \\
\hline $\mathrm{C}(2)-\mathrm{C}(1)-\mathrm{H}(1 \mathrm{~A})$ & 109.9 \\
\hline $\mathrm{C}(5)-\mathrm{C}(1)-\mathrm{H}(1 \mathrm{~B})$ & 109.9 \\
\hline $\mathrm{C}(2)-\mathrm{C}(1)-\mathrm{H}(1 \mathrm{~B})$ & 109.9 \\
\hline $\mathrm{H}(1 \mathrm{~A})-\mathrm{C}(1)-\mathrm{H}(1 \mathrm{~B})$ & 108.3 \\
\hline $\mathrm{C}(4)-\mathrm{C}(2)-\mathrm{C}(3)$ & $109.5(2)$ \\
\hline $\mathrm{C}(4)-\mathrm{C}(2)-\mathrm{C}(1)$ & $110.33(19)$ \\
\hline $\mathrm{C}(3)-\mathrm{C}(2)-\mathrm{C}(1)$ & $108.9(2)$ \\
\hline $\mathrm{C}(4)-\mathrm{C}(2)-\mathrm{H}(2)$ & 109.3 \\
\hline $\mathrm{C}(3)-\mathrm{C}(2)-\mathrm{H}(2)$ & 109.3 \\
\hline $\mathrm{C}(1)-\mathrm{C}(2)-\mathrm{H}(2)$ & 109.3 \\
\hline $\mathrm{C}(2)-\mathrm{C}(3)-\mathrm{C}(8)$ & $110.21(18)$ \\
\hline $\mathrm{C}(2)-\mathrm{C}(3)-\mathrm{H}(3 \mathrm{~A})$ & 109.6 \\
\hline $\mathrm{C}(8)-\mathrm{C}(3)-\mathrm{H}(3 \mathrm{~A})$ & 109.6 \\
\hline $\mathrm{C}(2)-\mathrm{C}(3)-\mathrm{H}(3 \mathrm{~B})$ & 109.6 \\
\hline $\mathrm{C}(8)-\mathrm{C}(3)-\mathrm{H}(3 \mathrm{~B})$ & 109.6 \\
\hline $\mathrm{H}(3 \mathrm{~A})-\mathrm{C}(3)-\mathrm{H}(3 \mathrm{~B})$ & 108.1 \\
\hline$C(2)-C(4)-C(6)$ & 109.47(19) \\
\hline $\mathrm{C}(2)-\mathrm{C}(4)-\mathrm{H}(4 \mathrm{~A})$ & 109.8 \\
\hline $\mathrm{C}(6)-\mathrm{C}(4)-\mathrm{H}(4 \mathrm{~A})$ & 109.8 \\
\hline $\mathrm{C}(2)-\mathrm{C}(4)-\mathrm{H}(4 \mathrm{~B})$ & 109.8 \\
\hline $\mathrm{C}(6)-\mathrm{C}(4)-\mathrm{H}(4 \mathrm{~B})$ & 109.8 \\
\hline $\mathrm{H}(4 \mathrm{~A})-\mathrm{C}(4)-\mathrm{H}(4 \mathrm{~B})$ & 108.2 \\
\hline
\end{tabular}




\begin{tabular}{|c|c|}
\hline$C(1)-C(5)-C(10)$ & $110.42(19)$ \\
\hline $\mathrm{C}(1)-\mathrm{C}(5)-\mathrm{C}(9)$ & $111.35(18)$ \\
\hline$C(10)-C(5)-C(9)$ & $108.77(18)$ \\
\hline $\mathrm{C}(1)-\mathrm{C}(5)-\mathrm{H}(5)$ & 108.7 \\
\hline $\mathrm{C}(10)-\mathrm{C}(5)-\mathrm{H}(5)$ & 108.7 \\
\hline $\mathrm{C}(9)-\mathrm{C}(5)-\mathrm{H}(5)$ & 108.7 \\
\hline$C(4)-C(6)-C(10)$ & 109.71(19) \\
\hline$C(4)-C(6)-C(7)$ & $111.96(18)$ \\
\hline$C(10)-C(6)-C(7)$ & $108.55(19)$ \\
\hline $\mathrm{C}(4)-\mathrm{C}(6)-\mathrm{H}(6)$ & 108.9 \\
\hline $\mathrm{C}(10)-\mathrm{C}(6)-\mathrm{H}(6)$ & 108.9 \\
\hline $\mathrm{C}(7)-\mathrm{C}(6)-\mathrm{H}(6)$ & 108.9 \\
\hline$C(14)-C(7)-C(6)$ & $108.53(19)$ \\
\hline $\mathrm{C}(14)-\mathrm{C}(7)-\mathrm{C}(8)$ & $112.38(19)$ \\
\hline$C(6)-C(7)-C(8)$ & $107.95(18)$ \\
\hline $\mathrm{C}(14)-\mathrm{C}(7)-\mathrm{H}(7)$ & 109.3 \\
\hline $\mathrm{C}(6)-\mathrm{C}(7)-\mathrm{H}(7)$ & 109.3 \\
\hline $\mathrm{C}(8)-\mathrm{C}(7)-\mathrm{H}(7)$ & 109.3 \\
\hline$C(3)-C(8)-C(7)$ & $110.31(19)$ \\
\hline $\mathrm{C}(3)-\mathrm{C}(8)-\mathrm{C}(9)$ & $110.47(19)$ \\
\hline$C(7)-C(8)-C(9)$ & $107.30(17)$ \\
\hline $\mathrm{C}(3)-\mathrm{C}(8)-\mathrm{P}(1)$ & $108.35(15)$ \\
\hline $\mathrm{C}(7)-\mathrm{C}(8)-\mathrm{P}(1)$ & $109.98(15)$ \\
\hline $\mathrm{C}(9)-\mathrm{C}(8)-\mathrm{P}(1)$ & $110.44(15)$ \\
\hline$C(12)-C(9)-C(5)$ & $108.95(18)$ \\
\hline $\mathrm{C}(12)-\mathrm{C}(9)-\mathrm{C}(8)$ & $112.54(18)$ \\
\hline$C(5)-C(9)-C(8)$ & $107.35(18)$ \\
\hline $\mathrm{C}(12)-\mathrm{C}(9)-\mathrm{H}(9)$ & 109.3 \\
\hline $\mathrm{C}(5)-\mathrm{C}(9)-\mathrm{H}(9)$ & 109.3 \\
\hline $\mathrm{C}(8)-\mathrm{C}(9)-\mathrm{H}(9)$ & 109.3 \\
\hline$C(6)-C(10)-C(11)$ & $111.58(19)$ \\
\hline$C(6)-C(10)-C(5)$ & $108.51(18)$ \\
\hline$C(11)-C(10)-C(5)$ & $110.98(19)$ \\
\hline $\mathrm{C}(6)-\mathrm{C}(10)-\mathrm{H}(10)$ & 108.6 \\
\hline $\mathrm{C}(11)-\mathrm{C}(10)-\mathrm{H}(10)$ & 108.6 \\
\hline $\mathrm{C}(5)-\mathrm{C}(10)-\mathrm{H}(10)$ & 108.6 \\
\hline$C(13)-C(11)-C(10)$ & 106.91(19) \\
\hline $\mathrm{C}(13)-\mathrm{C}(11)-\mathrm{H}(11 \mathrm{~A})$ & 110.3 \\
\hline $\mathrm{C}(10)-\mathrm{C}(11)-\mathrm{H}(11 \mathrm{~A})$ & 110.3 \\
\hline $\mathrm{C}(13)-\mathrm{C}(11)-\mathrm{H}(11 \mathrm{~B})$ & 110.3 \\
\hline $\mathrm{C}(10)-\mathrm{C}(11)-\mathrm{H}(11 \mathrm{~B})$ & 110.3 \\
\hline
\end{tabular}




\begin{tabular}{ll}
$\mathrm{H}(11 \mathrm{~A})-\mathrm{C}(11)-\mathrm{H}(11 \mathrm{~B})$ & 108.6 \\
$\mathrm{C}(13)-\mathrm{C}(12)-\mathrm{C}(9)$ & $108.65(18)$ \\
$\mathrm{C}(13)-\mathrm{C}(12)-\mathrm{H}(12 \mathrm{~A})$ & 110.0 \\
$\mathrm{C}(9)-\mathrm{C}(12)-\mathrm{H}(12 \mathrm{~A})$ & 110.0 \\
$\mathrm{C}(13)-\mathrm{C}(12)-\mathrm{H}(12 \mathrm{~B})$ & 110.0 \\
$\mathrm{C}(9)-\mathrm{C}(12)-\mathrm{H}(12 \mathrm{~B})$ & 110.0 \\
$\mathrm{H}(12 \mathrm{~A})-\mathrm{C}(12)-\mathrm{H}(12 \mathrm{~B})$ & 108.3 \\
$\mathrm{O}(1)-\mathrm{C}(13)-\mathrm{C}(11)$ & $112.50(18)$ \\
$\mathrm{O}(1)-\mathrm{C}(13)-\mathrm{C}(12)$ & $112.68(19)$ \\
$\mathrm{C}(11)-\mathrm{C}(13)-\mathrm{C}(12)$ & $111.2(2)$ \\
$\mathrm{O}(1)-\mathrm{C}(13)-\mathrm{C}(14)$ & $99.32(18)$ \\
$\mathrm{C}(11)-\mathrm{C}(13)-\mathrm{C}(14)$ & $109.8(2)$ \\
$\mathrm{C}(12)-\mathrm{C}(13)-\mathrm{C}(14)$ & $110.73(19)$ \\
$\mathrm{C}(13)-\mathrm{C}(14)-\mathrm{C}(7)$ & $109.47(19)$ \\
$\mathrm{C}(13)-\mathrm{C}(14)-\mathrm{H}(14 \mathrm{~A})$ & 109.8 \\
$\mathrm{C}(7)-\mathrm{C}(14)-\mathrm{H}(14 \mathrm{~A})$ & 109.8 \\
$\mathrm{C}(13)-\mathrm{C}(14)-\mathrm{H}(14 \mathrm{~B})$ & 109.8 \\
$\mathrm{C}(7)-\mathrm{C}(14)-\mathrm{H}(14 \mathrm{~B})$ & 109.8 \\
$\mathrm{H}(14 \mathrm{~A})-\mathrm{C}(14)-\mathrm{H}(14 \mathrm{~B})$ & 108.2 \\
\hline & \\
\hline
\end{tabular}


(24) (6-Hydroxydiamant-1-yl)phosphonic dichloride

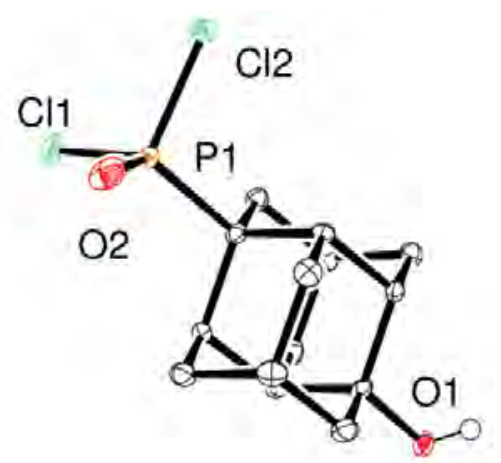

Ellipsoids are drawn at $50 \%$ probability.

Table S18. Crystal data and structure refinement for Compound 24.

CCDC No.

Empirical formula

Formula weight

Temperature

Wavelength

Crystal system

Space group

Unit cell dimensions

Volume

Z

Density (calculated)

Absorption coefficient

$\mathrm{F}(000)$

Crystal size

Theta range for data collection

Index ranges

Reflections collected

Independent reflections

Completeness to theta $=25.027^{\circ}$

Absorption correction

Max. and min. transmission

Refinement method

Data / restraints / parameters

Goodness-of-fit on $\mathrm{F}^{2}$

Final $\mathrm{R}$ indices $[\mathrm{I}>2 \operatorname{sigma}(\mathrm{I})]$

$\mathrm{R}$ indices (all data)
1053887

C14 H19 Cl2 O2 P

321.16

100(2) K

$0.71073 \AA$

Triclinic

$\mathrm{P}-1$

$\mathrm{a}=14.3021(5) \AA \quad \alpha=63.882(2)^{\circ}$.

$\mathrm{b}=14.7045(5) \AA \quad \beta=82.018(2)^{\circ}$.

$\mathrm{c}=15.0199(6) \AA \quad \gamma=89.034(2)^{\circ}$.

2805.26(18) $\AA^{3}$

8

$1.521 \mathrm{Mg} / \mathrm{m}^{3}$

$0.571 \mathrm{~mm}^{-1}$

1344

$0.479 \times 0.268 \times 0.162 \mathrm{~mm}^{3}$

2.163 to $25.027^{\circ}$.

$-17<=\mathrm{h}<=17,-17<=\mathrm{k}<=17,-17<=1<=17$

211520

$9906[\mathrm{R}($ int $)=0.0593]$

$99.9 \%$

Mulstiscan

0.7457 and 0.6737

Full-matrix least-squares on $\mathrm{F}^{2}$

9906 / 0 / 693

1.065

$\mathrm{R} 1=0.0315, \mathrm{wR} 2=0.0736$

$\mathrm{R} 1=0.0360, \mathrm{wR} 2=0.0757$ 
Extinction coefficient

Largest diff. peak and hole $\mathrm{n} / \mathrm{a}$

0.384 and -0.296 e. $\AA^{-3}$ 
Table S19. Bond lengths $[\AA]$ and angles $\left[^{\circ}\right]$ for Compound 24.

\begin{tabular}{|c|c|}
\hline $\mathrm{Cl}(1)-\mathrm{P}(1)$ & $2.0141(7)$ \\
\hline $\mathrm{O}(1)-\mathrm{C}(7)$ & $1.439(2)$ \\
\hline $\mathrm{O}(1)-\mathrm{H}(1)$ & $0.75(3)$ \\
\hline $\mathrm{P}(1)-\mathrm{O}(2)$ & $1.4548(15)$ \\
\hline$P(1)-C(10)$ & $1.8377(18)$ \\
\hline $\mathrm{P}(1)-\mathrm{Cl}(2)$ & $2.0286(7)$ \\
\hline$C(1)-C(7)$ & $1.521(3)$ \\
\hline$C(1)-C(2)$ & $1.531(3)$ \\
\hline $\mathrm{C}(1)-\mathrm{H}(1 \mathrm{~A})$ & 0.9900 \\
\hline $\mathrm{C}(1)-\mathrm{H}(1 \mathrm{~B})$ & 0.9900 \\
\hline $\mathrm{P}(2)-\mathrm{O}(3)$ & $1.4571(15)$ \\
\hline $\mathrm{P}(2)-\mathrm{C}(23)$ & $1.8337(18)$ \\
\hline $\mathrm{P}(2)-\mathrm{Cl}(3)$ & $2.0142(7)$ \\
\hline $\mathrm{P}(2)-\mathrm{Cl}(4)$ & $2.0299(7)$ \\
\hline $\mathrm{C}(2)-\mathrm{C}(3)$ & $1.532(3)$ \\
\hline $\mathrm{C}(2)-\mathrm{C}(4)$ & $1.537(3)$ \\
\hline $\mathrm{C}(2)-\mathrm{H}(2)$ & 1.0000 \\
\hline $\mathrm{P}(3)-\mathrm{O}(6)$ & $1.4574(15)$ \\
\hline $\mathrm{P}(3)-\mathrm{C}(33)$ & $1.8359(18)$ \\
\hline $\mathrm{P}(3)-\mathrm{Cl}(5)$ & $2.0137(7)$ \\
\hline $\mathrm{P}(3)-\mathrm{Cl}(6)$ & $2.0303(7)$ \\
\hline$C(3)-C(5)$ & $1.536(3)$ \\
\hline $\mathrm{C}(3)-\mathrm{H}(3 \mathrm{~A})$ & 0.9900 \\
\hline $\mathrm{C}(3)-\mathrm{H}(3 \mathrm{~B})$ & 0.9900 \\
\hline$C(4)-C(9)$ & $1.537(3)$ \\
\hline $\mathrm{C}(4)-\mathrm{H}(4 \mathrm{~A})$ & 0.9900 \\
\hline $\mathrm{C}(4)-\mathrm{H}(4 \mathrm{~B})$ & 0.9900 \\
\hline $\mathrm{P}(4)-\mathrm{O}(8)$ & $1.4558(17)$ \\
\hline $\mathrm{P}(4)-\mathrm{C}(46)$ & $1.8367(19)$ \\
\hline $\mathrm{P}(4)-\mathrm{Cl}(7)$ & $2.0106(8)$ \\
\hline $\mathrm{P}(4)-\mathrm{Cl}(8)$ & $2.0270(8)$ \\
\hline $\mathrm{O}(4)-\mathrm{C}(21)$ & $1.440(2)$ \\
\hline $\mathrm{O}(4)-\mathrm{H}(4)$ & $0.79(3)$ \\
\hline $\mathrm{O}(5)-\mathrm{C}(42)$ & $1.441(2)$ \\
\hline $\mathrm{O}(5)-\mathrm{H}(5 \mathrm{~A})$ & $0.79(3)$ \\
\hline$C(5)-C(6)$ & $1.549(2)$ \\
\hline$C(5)-C(10)$ & $1.562(2)$ \\
\hline $\mathrm{C}(5)-\mathrm{H}(5)$ & 1.0000 \\
\hline
\end{tabular}




\begin{tabular}{|c|c|}
\hline$C(6)-C(12)$ & $1.527(3)$ \\
\hline$C(6)-C(7)$ & $1.536(3)$ \\
\hline $\mathrm{C}(6)-\mathrm{H}(6)$ & 1.0000 \\
\hline $\mathrm{O}(7)-\mathrm{C}(53)$ & $1.437(2)$ \\
\hline $\mathrm{O}(7)-\mathrm{H}(7)$ & $0.79(3)$ \\
\hline$C(7)-C(8)$ & $1.543(3)$ \\
\hline$C(8)-C(13)$ & $1.528(3)$ \\
\hline $\mathrm{C}(8)-\mathrm{C}(9)$ & $1.552(2)$ \\
\hline $\mathrm{C}(8)-\mathrm{H}(8)$ & 1.0000 \\
\hline C(9)-C(10) & $1.552(3)$ \\
\hline $\mathrm{C}(9)-\mathrm{H}(9)$ & 1.0000 \\
\hline$C(10)-C(11)$ & $1.547(3)$ \\
\hline $\mathrm{C}(12)-\mathrm{C}(14)$ & $1.527(3)$ \\
\hline $\mathrm{C}(12)-\mathrm{H}(12 \mathrm{~A})$ & 0.9900 \\
\hline $\mathrm{C}(12)-\mathrm{H}(12 \mathrm{~B})$ & 0.9900 \\
\hline $\mathrm{C}(11)-\mathrm{C}(14)$ & $1.527(3)$ \\
\hline $\mathrm{C}(11)-\mathrm{H}(11 \mathrm{~A})$ & 0.9900 \\
\hline $\mathrm{C}(11)-\mathrm{H}(11 \mathrm{~B})$ & 0.9900 \\
\hline$C(13)-C(14)$ & $1.528(3)$ \\
\hline $\mathrm{C}(13)-\mathrm{H}(13 \mathrm{~A})$ & 0.9900 \\
\hline $\mathrm{C}(13)-\mathrm{H}(13 \mathrm{~B})$ & 0.9900 \\
\hline $\mathrm{C}(14)-\mathrm{H}(14)$ & 1.0000 \\
\hline$C(15)-C(16)$ & $1.526(3)$ \\
\hline$C(15)-C(20)$ & $1.529(3)$ \\
\hline $\mathrm{C}(15)-\mathrm{H}(15 \mathrm{~A})$ & 0.9900 \\
\hline $\mathrm{C}(15)-\mathrm{H}(15 \mathrm{~B})$ & 0.9900 \\
\hline$C(17)-C(18)$ & $1.528(3)$ \\
\hline$C(17)-C(16)$ & $1.528(3)$ \\
\hline $\mathrm{C}(17)-\mathrm{H}(17 \mathrm{~A})$ & 0.9900 \\
\hline $\mathrm{C}(17)-\mathrm{H}(17 \mathrm{~B})$ & 0.9900 \\
\hline$C(16)-C(19)$ & $1.526(3)$ \\
\hline $\mathrm{C}(16)-\mathrm{H}(16)$ & 1.0000 \\
\hline $\mathrm{C}(18)-\mathrm{C}(21)$ & $1.541(3)$ \\
\hline $\mathrm{C}(18)-\mathrm{C}(22)$ & $1.556(3)$ \\
\hline $\mathrm{C}(18)-\mathrm{H}(18)$ & 1.0000 \\
\hline$C(19)-C(23)$ & $1.548(2)$ \\
\hline C(19)-H(19A) & 0.9900 \\
\hline $\mathrm{C}(19)-\mathrm{H}(19 \mathrm{~B})$ & 0.9900 \\
\hline$C(20)-C(21)$ & $1.531(3)$ \\
\hline $\mathrm{C}(20)-\mathrm{C}(24)$ & $1.548(2)$ \\
\hline $\mathrm{C}(20)-\mathrm{H}(20)$ & 1.0000 \\
\hline
\end{tabular}




\begin{tabular}{|c|c|}
\hline $\mathrm{C}(21)-\mathrm{C}(25)$ & $1.521(3)$ \\
\hline$C(22)-C(26)$ & $1.537(3)$ \\
\hline$C(22)-C(23)$ & $1.557(2)$ \\
\hline $\mathrm{C}(22)-\mathrm{H}(22)$ & 1.0000 \\
\hline $\mathrm{C}(23)-\mathrm{C}(24)$ & $1.560(2)$ \\
\hline $\mathrm{C}(24)-\mathrm{C}(28)$ & $1.535(3)$ \\
\hline $\mathrm{C}(24)-\mathrm{H}(24)$ & 1.0000 \\
\hline$C(25)-C(27)$ & $1.532(3)$ \\
\hline $\mathrm{C}(25)-\mathrm{H}(25 \mathrm{~A})$ & 0.9900 \\
\hline $\mathrm{C}(25)-\mathrm{H}(25 \mathrm{~B})$ & 0.9900 \\
\hline $\mathrm{C}(26)-\mathrm{C}(27)$ & $1.536(3)$ \\
\hline $\mathrm{C}(26)-\mathrm{H}(26 \mathrm{~A})$ & 0.9900 \\
\hline $\mathrm{C}(26)-\mathrm{H}(26 \mathrm{~B})$ & 0.9900 \\
\hline$C(27)-C(28)$ & $1.533(3)$ \\
\hline $\mathrm{C}(27)-\mathrm{H}(27)$ & 1.0000 \\
\hline $\mathrm{C}(28)-\mathrm{H}(28 \mathrm{~A})$ & 0.9900 \\
\hline $\mathrm{C}(28)-\mathrm{H}(28 \mathrm{~B})$ & 0.9900 \\
\hline $\mathrm{C}(29)-\mathrm{C}(35)$ & $1.527(3)$ \\
\hline$C(29)-C(30)$ & $1.530(3)$ \\
\hline $\mathrm{C}(29)-\mathrm{H}(29 \mathrm{~A})$ & 0.9900 \\
\hline $\mathrm{C}(29)-\mathrm{H}(29 \mathrm{~B})$ & 0.9900 \\
\hline $\mathrm{C}(30)-\mathrm{C}(31)$ & $1.525(3)$ \\
\hline $\mathrm{C}(30)-\mathrm{C}(32)$ & $1.530(3)$ \\
\hline $\mathrm{C}(30)-\mathrm{H}(30)$ & 1.0000 \\
\hline $\mathrm{C}(31)-\mathrm{C}(34)$ & $1.524(3)$ \\
\hline $\mathrm{C}(31)-\mathrm{H}(31 \mathrm{~A})$ & 0.9900 \\
\hline $\mathrm{C}(31)-\mathrm{H}(31 \mathrm{~B})$ & 0.9900 \\
\hline $\mathrm{C}(32)-\mathrm{C}(33)$ & $1.547(3)$ \\
\hline $\mathrm{C}(32)-\mathrm{H}(32 \mathrm{~A})$ & 0.9900 \\
\hline $\mathrm{C}(32)-\mathrm{H}(32 \mathrm{~B})$ & 0.9900 \\
\hline $\mathrm{C}(33)-\mathrm{C}(37)$ & $1.557(2)$ \\
\hline $\mathrm{C}(33)-\mathrm{C}(36)$ & $1.559(3)$ \\
\hline$C(34)-C(42)$ & $1.538(3)$ \\
\hline $\mathrm{C}(34)-\mathrm{C}(37)$ & $1.551(2)$ \\
\hline $\mathrm{C}(34)-\mathrm{H}(34)$ & 1.0000 \\
\hline $\mathrm{C}(35)-\mathrm{C}(42)$ & $1.538(3)$ \\
\hline$C(35)-C(36)$ & $1.552(2)$ \\
\hline $\mathrm{C}(35)-\mathrm{H}(35)$ & 1.0000 \\
\hline $\mathrm{C}(36)-\mathrm{C}(38)$ & $1.534(3)$ \\
\hline $\mathrm{C}(36)-\mathrm{H}(36)$ & 1.0000 \\
\hline C(37)-C(39) & $1.533(3)$ \\
\hline
\end{tabular}




\begin{tabular}{|c|c|}
\hline $\mathrm{C}(37)-\mathrm{H}(37)$ & 1.0000 \\
\hline $\mathrm{C}(38)-\mathrm{C}(40)$ & $1.529(3)$ \\
\hline $\mathrm{C}(38)-\mathrm{H}(38 \mathrm{~A})$ & 0.9900 \\
\hline $\mathrm{C}(38)-\mathrm{H}(38 \mathrm{~B})$ & 0.9900 \\
\hline$C(39)-C(40)$ & $1.529(3)$ \\
\hline $\mathrm{C}(39)-\mathrm{H}(39 \mathrm{~A})$ & 0.9900 \\
\hline $\mathrm{C}(39)-\mathrm{H}(39 \mathrm{~B})$ & 0.9900 \\
\hline $\mathrm{C}(40)-\mathrm{C}(41)$ & $1.529(3)$ \\
\hline $\mathrm{C}(40)-\mathrm{H}(40)$ & 1.0000 \\
\hline$C(41)-C(42)$ & $1.520(3)$ \\
\hline $\mathrm{C}(41)-\mathrm{H}(41 \mathrm{~A})$ & 0.9900 \\
\hline $\mathrm{C}(41)-\mathrm{H}(41 \mathrm{~B})$ & 0.9900 \\
\hline$C(43)-C(48)$ & $1.525(3)$ \\
\hline $\mathrm{C}(43)-\mathrm{C}(44)$ & $1.528(3)$ \\
\hline $\mathrm{C}(43)-\mathrm{H}(43 \mathrm{~A})$ & 0.9900 \\
\hline $\mathrm{C}(43)-\mathrm{H}(43 \mathrm{~B})$ & 0.9900 \\
\hline$C(44)-C(45)$ & $1.525(3)$ \\
\hline$C(44)-C(50)$ & $1.525(3)$ \\
\hline $\mathrm{C}(44)-\mathrm{H}(44)$ & 1.0000 \\
\hline$C(45)-C(46)$ & $1.546(3)$ \\
\hline $\mathrm{C}(45)-\mathrm{H}(45 \mathrm{~A})$ & 0.9900 \\
\hline $\mathrm{C}(45)-\mathrm{H}(45 \mathrm{~B})$ & 0.9900 \\
\hline $\mathrm{C}(46)-\mathrm{C}(47)$ & $1.555(3)$ \\
\hline $\mathrm{C}(46)-\mathrm{C}(51)$ & $1.555(2)$ \\
\hline C(47)-C(49) & $1.535(3)$ \\
\hline $\mathrm{C}(47)-\mathrm{C}(48)$ & $1.548(3)$ \\
\hline $\mathrm{C}(47)-\mathrm{H}(47)$ & 1.0000 \\
\hline $\mathrm{C}(48)-\mathrm{C}(53)$ & $1.535(3)$ \\
\hline $\mathrm{C}(48)-\mathrm{H}(48)$ & 1.0000 \\
\hline $\mathrm{C}(49)-\mathrm{C}(55)$ & $1.533(3)$ \\
\hline $\mathrm{C}(49)-\mathrm{H}(49 \mathrm{~A})$ & 0.9900 \\
\hline $\mathrm{C}(49)-\mathrm{H}(49 \mathrm{~B})$ & 0.9900 \\
\hline$C(50)-C(52)$ & $1.522(3)$ \\
\hline $\mathrm{C}(50)-\mathrm{H}(50 \mathrm{~A})$ & 0.9900 \\
\hline $\mathrm{C}(50)-\mathrm{H}(50 \mathrm{~B})$ & 0.9900 \\
\hline$C(51)-C(56)$ & $1.532(3)$ \\
\hline $\mathrm{C}(51)-\mathrm{C}(52)$ & $1.549(3)$ \\
\hline $\mathrm{C}(51)-\mathrm{H}(51)$ & 1.0000 \\
\hline$C(52)-C(53)$ & $1.542(3)$ \\
\hline $\mathrm{C}(52)-\mathrm{H}(52)$ & 1.0000 \\
\hline$C(53)-C(54)$ & $1.521(3)$ \\
\hline
\end{tabular}




\begin{tabular}{|c|c|}
\hline$C(54)-C(55)$ & $1.529(3)$ \\
\hline $\mathrm{C}(54)-\mathrm{H}(54 \mathrm{~A})$ & 0.9900 \\
\hline $\mathrm{C}(54)-\mathrm{H}(54 \mathrm{~B})$ & 0.9900 \\
\hline$C(55)-C(56)$ & $1.531(3)$ \\
\hline $\mathrm{C}(55)-\mathrm{H}(55)$ & 1.0000 \\
\hline $\mathrm{C}(56)-\mathrm{H}(56 \mathrm{~A})$ & 0.9900 \\
\hline $\mathrm{C}(56)-\mathrm{H}(56 \mathrm{~B})$ & 0.9900 \\
\hline $\mathrm{C}(7)-\mathrm{O}(1)-\mathrm{H}(1)$ & 109.5 \\
\hline $\mathrm{O}(2)-\mathrm{P}(1)-\mathrm{C}(10)$ & $120.96(9)$ \\
\hline $\mathrm{O}(2)-\mathrm{P}(1)-\mathrm{Cl}(1)$ & $111.30(7)$ \\
\hline $\mathrm{C}(10)-\mathrm{P}(1)-\mathrm{Cl}(1)$ & $106.06(6)$ \\
\hline $\mathrm{O}(2)-\mathrm{P}(1)-\mathrm{Cl}(2)$ & $110.72(7)$ \\
\hline $\mathrm{C}(10)-\mathrm{P}(1)-\mathrm{Cl}(2)$ & $104.21(6)$ \\
\hline $\mathrm{Cl}(1)-\mathrm{P}(1)-\mathrm{Cl}(2)$ & $101.69(3)$ \\
\hline $\mathrm{C}(7)-\mathrm{C}(1)-\mathrm{C}(2)$ & $110.04(15)$ \\
\hline $\mathrm{C}(7)-\mathrm{C}(1)-\mathrm{H}(1 \mathrm{~A})$ & 109.7 \\
\hline $\mathrm{C}(2)-\mathrm{C}(1)-\mathrm{H}(1 \mathrm{~A})$ & 109.7 \\
\hline $\mathrm{C}(7)-\mathrm{C}(1)-\mathrm{H}(1 \mathrm{~B})$ & 109.7 \\
\hline $\mathrm{C}(2)-\mathrm{C}(1)-\mathrm{H}(1 \mathrm{~B})$ & 109.7 \\
\hline $\mathrm{H}(1 \mathrm{~A})-\mathrm{C}(1)-\mathrm{H}(1 \mathrm{~B})$ & 108.2 \\
\hline $\mathrm{O}(3)-\mathrm{P}(2)-\mathrm{C}(23)$ & $121.05(8)$ \\
\hline $\mathrm{O}(3)-\mathrm{P}(2)-\mathrm{Cl}(3)$ & $111.60(7)$ \\
\hline $\mathrm{C}(23)-\mathrm{P}(2)-\mathrm{Cl}(3)$ & $105.48(6)$ \\
\hline $\mathrm{O}(3)-\mathrm{P}(2)-\mathrm{Cl}(4)$ & $110.34(7)$ \\
\hline $\mathrm{C}(23)-\mathrm{P}(2)-\mathrm{Cl}(4)$ & $104.70(6)$ \\
\hline $\mathrm{Cl}(3)-\mathrm{P}(2)-\mathrm{Cl}(4)$ & $101.78(3)$ \\
\hline $\mathrm{C}(1)-\mathrm{C}(2)-\mathrm{C}(3)$ & $108.87(16)$ \\
\hline$C(1)-C(2)-C(4)$ & $108.76(16)$ \\
\hline $\mathrm{C}(3)-\mathrm{C}(2)-\mathrm{C}(4)$ & $110.32(16)$ \\
\hline $\mathrm{C}(1)-\mathrm{C}(2)-\mathrm{H}(2)$ & 109.6 \\
\hline $\mathrm{C}(3)-\mathrm{C}(2)-\mathrm{H}(2)$ & 109.6 \\
\hline $\mathrm{C}(4)-\mathrm{C}(2)-\mathrm{H}(2)$ & 109.6 \\
\hline $\mathrm{O}(6)-\mathrm{P}(3)-\mathrm{C}(33)$ & $121.33(8)$ \\
\hline $\mathrm{O}(6)-\mathrm{P}(3)-\mathrm{Cl}(5)$ & $111.08(7)$ \\
\hline $\mathrm{C}(33)-\mathrm{P}(3)-\mathrm{Cl}(5)$ & $105.77(6)$ \\
\hline $\mathrm{O}(6)-\mathrm{P}(3)-\mathrm{Cl}(6)$ & $109.83(7)$ \\
\hline $\mathrm{C}(33)-\mathrm{P}(3)-\mathrm{Cl}(6)$ & $104.77(6)$ \\
\hline $\mathrm{Cl}(5)-\mathrm{P}(3)-\mathrm{Cl}(6)$ & $102.25(3)$ \\
\hline$C(2)-C(3)-C(5)$ & $109.91(15)$ \\
\hline $\mathrm{C}(2)-\mathrm{C}(3)-\mathrm{H}(3 \mathrm{~A})$ & 109.7 \\
\hline
\end{tabular}




\begin{tabular}{|c|c|}
\hline $\mathrm{C}(5)-\mathrm{C}(3)-\mathrm{H}(3 \mathrm{~A})$ & 109.7 \\
\hline $\mathrm{C}(2)-\mathrm{C}(3)-\mathrm{H}(3 \mathrm{~B})$ & 109.7 \\
\hline $\mathrm{C}(5)-\mathrm{C}(3)-\mathrm{H}(3 \mathrm{~B})$ & 109.7 \\
\hline $\mathrm{H}(3 \mathrm{~A})-\mathrm{C}(3)-\mathrm{H}(3 \mathrm{~B})$ & 108.2 \\
\hline$C(2)-C(4)-C(9)$ & $109.74(15)$ \\
\hline $\mathrm{C}(2)-\mathrm{C}(4)-\mathrm{H}(4 \mathrm{~A})$ & 109.7 \\
\hline $\mathrm{C}(9)-\mathrm{C}(4)-\mathrm{H}(4 \mathrm{~A})$ & 109.7 \\
\hline $\mathrm{C}(2)-\mathrm{C}(4)-\mathrm{H}(4 \mathrm{~B})$ & 109.7 \\
\hline $\mathrm{C}(9)-\mathrm{C}(4)-\mathrm{H}(4 \mathrm{~B})$ & 109.7 \\
\hline $\mathrm{H}(4 \mathrm{~A})-\mathrm{C}(4)-\mathrm{H}(4 \mathrm{~B})$ & 108.2 \\
\hline $\mathrm{O}(8)-\mathrm{P}(4)-\mathrm{C}(46)$ & $120.79(9)$ \\
\hline $\mathrm{O}(8)-\mathrm{P}(4)-\mathrm{Cl}(7)$ & $111.44(8)$ \\
\hline $\mathrm{C}(46)-\mathrm{P}(4)-\mathrm{Cl}(7)$ & $105.69(6)$ \\
\hline $\mathrm{O}(8)-\mathrm{P}(4)-\mathrm{Cl}(8)$ & $110.18(8)$ \\
\hline $\mathrm{C}(46)-\mathrm{P}(4)-\mathrm{Cl}(8)$ & $104.64(7)$ \\
\hline $\mathrm{Cl}(7)-\mathrm{P}(4)-\mathrm{Cl}(8)$ & $102.36(3)$ \\
\hline $\mathrm{C}(21)-\mathrm{O}(4)-\mathrm{H}(4)$ & 109.5 \\
\hline $\mathrm{C}(42)-\mathrm{O}(5)-\mathrm{H}(5 \mathrm{~A})$ & 109.5 \\
\hline$C(3)-C(5)-C(6)$ & $109.41(15)$ \\
\hline$C(3)-C(5)-C(10)$ & $112.03(15)$ \\
\hline$C(6)-C(5)-C(10)$ & $107.23(14)$ \\
\hline $\mathrm{C}(3)-\mathrm{C}(5)-\mathrm{H}(5)$ & 109.4 \\
\hline $\mathrm{C}(6)-\mathrm{C}(5)-\mathrm{H}(5)$ & 109.4 \\
\hline $\mathrm{C}(10)-\mathrm{C}(5)-\mathrm{H}(5)$ & 109.4 \\
\hline$C(12)-C(6)-C(7)$ & $110.76(15)$ \\
\hline$C(12)-C(6)-C(5)$ & $111.45(15)$ \\
\hline$C(7)-C(6)-C(5)$ & $109.42(15)$ \\
\hline $\mathrm{C}(12)-\mathrm{C}(6)-\mathrm{H}(6)$ & 108.4 \\
\hline $\mathrm{C}(7)-\mathrm{C}(6)-\mathrm{H}(6)$ & 108.4 \\
\hline $\mathrm{C}(5)-\mathrm{C}(6)-\mathrm{H}(6)$ & 108.4 \\
\hline $\mathrm{C}(53)-\mathrm{O}(7)-\mathrm{H}(7)$ & 109.5 \\
\hline $\mathrm{O}(1)-\mathrm{C}(7)-\mathrm{C}(1)$ & $109.57(15)$ \\
\hline $\mathrm{O}(1)-\mathrm{C}(7)-\mathrm{C}(6)$ & $106.83(15)$ \\
\hline$C(1)-C(7)-C(6)$ & $110.39(16)$ \\
\hline $\mathrm{O}(1)-\mathrm{C}(7)-\mathrm{C}(8)$ & $111.42(15)$ \\
\hline $\mathrm{C}(1)-\mathrm{C}(7)-\mathrm{C}(8)$ & $111.13(15)$ \\
\hline$C(6)-C(7)-C(8)$ & $107.39(15)$ \\
\hline $\mathrm{C}(13)-\mathrm{C}(8)-\mathrm{C}(7)$ & $110.66(15)$ \\
\hline $\mathrm{C}(13)-\mathrm{C}(8)-\mathrm{C}(9)$ & $111.67(15)$ \\
\hline$C(7)-C(8)-C(9)$ & $108.67(15)$ \\
\hline $\mathrm{C}(13)-\mathrm{C}(8)-\mathrm{H}(8)$ & 108.6 \\
\hline
\end{tabular}




\begin{tabular}{|c|c|}
\hline $\mathrm{C}(7)-\mathrm{C}(8)-\mathrm{H}(8)$ & 108.6 \\
\hline $\mathrm{C}(9)-\mathrm{C}(8)-\mathrm{H}(8)$ & 108.6 \\
\hline $\mathrm{C}(4)-\mathrm{C}(9)-\mathrm{C}(10)$ & $112.44(15)$ \\
\hline $\mathrm{C}(4)-\mathrm{C}(9)-\mathrm{C}(8)$ & $109.49(15)$ \\
\hline $\mathrm{C}(10)-\mathrm{C}(9)-\mathrm{C}(8)$ & $107.60(14)$ \\
\hline $\mathrm{C}(4)-\mathrm{C}(9)-\mathrm{H}(9)$ & 109.1 \\
\hline $\mathrm{C}(10)-\mathrm{C}(9)-\mathrm{H}(9)$ & 109.1 \\
\hline $\mathrm{C}(8)-\mathrm{C}(9)-\mathrm{H}(9)$ & 109.1 \\
\hline $\mathrm{C}(11)-\mathrm{C}(10)-\mathrm{C}(9)$ & $110.92(15)$ \\
\hline $\mathrm{C}(11)-\mathrm{C}(10)-\mathrm{C}(5)$ & $109.97(15)$ \\
\hline $\mathrm{C}(9)-\mathrm{C}(10)-\mathrm{C}(5)$ & $107.35(14)$ \\
\hline $\mathrm{C}(11)-\mathrm{C}(10)-\mathrm{P}(1)$ & $107.56(12)$ \\
\hline $\mathrm{C}(9)-\mathrm{C}(10)-\mathrm{P}(1)$ & $110.65(12)$ \\
\hline$C(5)-C(10)-P(1)$ & $110.41(12)$ \\
\hline$C(14)-C(12)-C(6)$ & $109.19(15)$ \\
\hline $\mathrm{C}(14)-\mathrm{C}(12)-\mathrm{H}(12 \mathrm{~A})$ & 109.8 \\
\hline $\mathrm{C}(6)-\mathrm{C}(12)-\mathrm{H}(12 \mathrm{~A})$ & 109.8 \\
\hline $\mathrm{C}(14)-\mathrm{C}(12)-\mathrm{H}(12 \mathrm{~B})$ & 109.8 \\
\hline $\mathrm{C}(6)-\mathrm{C}(12)-\mathrm{H}(12 \mathrm{~B})$ & 109.8 \\
\hline $\mathrm{H}(12 \mathrm{~A})-\mathrm{C}(12)-\mathrm{H}(12 \mathrm{~B})$ & 108.3 \\
\hline$C(14)-C(11)-C(10)$ & $110.48(15)$ \\
\hline $\mathrm{C}(14)-\mathrm{C}(11)-\mathrm{H}(11 \mathrm{~A})$ & 109.6 \\
\hline $\mathrm{C}(10)-\mathrm{C}(11)-\mathrm{H}(11 \mathrm{~A})$ & 109.6 \\
\hline $\mathrm{C}(14)-\mathrm{C}(11)-\mathrm{H}(11 \mathrm{~B})$ & 109.6 \\
\hline $\mathrm{C}(10)-\mathrm{C}(11)-\mathrm{H}(11 \mathrm{~B})$ & 109.6 \\
\hline $\mathrm{H}(11 \mathrm{~A})-\mathrm{C}(11)-\mathrm{H}(11 \mathrm{~B})$ & 108.1 \\
\hline$C(8)-C(13)-C(14)$ & $109.23(15)$ \\
\hline $\mathrm{C}(8)-\mathrm{C}(13)-\mathrm{H}(13 \mathrm{~A})$ & 109.8 \\
\hline $\mathrm{C}(14)-\mathrm{C}(13)-\mathrm{H}(13 \mathrm{~A})$ & 109.8 \\
\hline $\mathrm{C}(8)-\mathrm{C}(13)-\mathrm{H}(13 \mathrm{~B})$ & 109.8 \\
\hline $\mathrm{C}(14)-\mathrm{C}(13)-\mathrm{H}(13 \mathrm{~B})$ & 109.8 \\
\hline $\mathrm{H}(13 \mathrm{~A})-\mathrm{C}(13)-\mathrm{H}(13 \mathrm{~B})$ & 108.3 \\
\hline$C(12)-C(14)-C(11)$ & $108.77(15)$ \\
\hline$C(12)-C(14)-C(13)$ & $110.39(16)$ \\
\hline$C(11)-C(14)-C(13)$ & 109.01(15) \\
\hline $\mathrm{C}(12)-\mathrm{C}(14)-\mathrm{H}(14)$ & 109.6 \\
\hline $\mathrm{C}(11)-\mathrm{C}(14)-\mathrm{H}(14)$ & 109.6 \\
\hline $\mathrm{C}(13)-\mathrm{C}(14)-\mathrm{H}(14)$ & 109.6 \\
\hline$C(16)-C(15)-C(20)$ & $109.34(15)$ \\
\hline $\mathrm{C}(16)-\mathrm{C}(15)-\mathrm{H}(15 \mathrm{~A})$ & 109.8 \\
\hline $\mathrm{C}(20)-\mathrm{C}(15)-\mathrm{H}(15 \mathrm{~A})$ & 109.8 \\
\hline
\end{tabular}




\begin{tabular}{|c|c|}
\hline $\mathrm{C}(16)-\mathrm{C}(15)-\mathrm{H}(15 \mathrm{~B})$ & 109.8 \\
\hline $\mathrm{C}(20)-\mathrm{C}(15)-\mathrm{H}(15 \mathrm{~B})$ & 109.8 \\
\hline $\mathrm{H}(15 \mathrm{~A})-\mathrm{C}(15)-\mathrm{H}(15 \mathrm{~B})$ & 108.3 \\
\hline $\mathrm{C}(18)-\mathrm{C}(17)-\mathrm{C}(16)$ & $109.14(15)$ \\
\hline $\mathrm{C}(18)-\mathrm{C}(17)-\mathrm{H}(17 \mathrm{~A})$ & 109.9 \\
\hline $\mathrm{C}(16)-\mathrm{C}(17)-\mathrm{H}(17 \mathrm{~A})$ & 109.9 \\
\hline C(18)-C(17)-H(17B) & 109.9 \\
\hline $\mathrm{C}(16)-\mathrm{C}(17)-\mathrm{H}(17 \mathrm{~B})$ & 109.9 \\
\hline $\mathrm{H}(17 \mathrm{~A})-\mathrm{C}(17)-\mathrm{H}(17 \mathrm{~B})$ & 108.3 \\
\hline$C(15)-C(16)-C(19)$ & $108.88(15)$ \\
\hline $\mathrm{C}(15)-\mathrm{C}(16)-\mathrm{C}(17)$ & $110.62(16)$ \\
\hline $\mathrm{C}(19)-\mathrm{C}(16)-\mathrm{C}(17)$ & $109.12(15)$ \\
\hline $\mathrm{C}(15)-\mathrm{C}(16)-\mathrm{H}(16)$ & 109.4 \\
\hline $\mathrm{C}(19)-\mathrm{C}(16)-\mathrm{H}(16)$ & 109.4 \\
\hline $\mathrm{C}(17)-\mathrm{C}(16)-\mathrm{H}(16)$ & 109.4 \\
\hline $\mathrm{C}(17)-\mathrm{C}(18)-\mathrm{C}(21)$ & $110.28(15)$ \\
\hline $\mathrm{C}(17)-\mathrm{C}(18)-\mathrm{C}(22)$ & $111.61(15)$ \\
\hline $\mathrm{C}(21)-\mathrm{C}(18)-\mathrm{C}(22)$ & $108.68(15)$ \\
\hline $\mathrm{C}(17)-\mathrm{C}(18)-\mathrm{H}(18)$ & 108.7 \\
\hline $\mathrm{C}(21)-\mathrm{C}(18)-\mathrm{H}(18)$ & 108.7 \\
\hline $\mathrm{C}(22)-\mathrm{C}(18)-\mathrm{H}(18)$ & 108.7 \\
\hline$C(16)-C(19)-C(23)$ & $110.07(15)$ \\
\hline $\mathrm{C}(16)-\mathrm{C}(19)-\mathrm{H}(19 \mathrm{~A})$ & 109.6 \\
\hline $\mathrm{C}(23)-\mathrm{C}(19)-\mathrm{H}(19 \mathrm{~A})$ & 109.6 \\
\hline C(16)-C(19)-H(19B) & 109.6 \\
\hline C(23)-C(19)-H(19B) & 109.6 \\
\hline H(19A)-C(19)-H(19B) & 108.2 \\
\hline$C(15)-C(20)-C(21)$ & $110.64(15)$ \\
\hline $\mathrm{C}(15)-\mathrm{C}(20)-\mathrm{C}(24)$ & $111.02(15)$ \\
\hline $\mathrm{C}(21)-\mathrm{C}(20)-\mathrm{C}(24)$ & $109.30(15)$ \\
\hline $\mathrm{C}(15)-\mathrm{C}(20)-\mathrm{H}(20)$ & 108.6 \\
\hline $\mathrm{C}(21)-\mathrm{C}(20)-\mathrm{H}(20)$ & 108.6 \\
\hline $\mathrm{C}(24)-\mathrm{C}(20)-\mathrm{H}(20)$ & 108.6 \\
\hline $\mathrm{O}(4)-\mathrm{C}(21)-\mathrm{C}(25)$ & $109.18(15)$ \\
\hline $\mathrm{O}(4)-\mathrm{C}(21)-\mathrm{C}(20)$ & $106.78(15)$ \\
\hline $\mathrm{C}(25)-\mathrm{C}(21)-\mathrm{C}(20)$ & $110.37(15)$ \\
\hline $\mathrm{O}(4)-\mathrm{C}(21)-\mathrm{C}(18)$ & $111.56(15)$ \\
\hline $\mathrm{C}(25)-\mathrm{C}(21)-\mathrm{C}(18)$ & $110.75(15)$ \\
\hline $\mathrm{C}(20)-\mathrm{C}(21)-\mathrm{C}(18)$ & $108.12(15)$ \\
\hline $\mathrm{C}(26)-\mathrm{C}(22)-\mathrm{C}(18)$ & $109.13(15)$ \\
\hline$C(26)-C(22)-C(23)$ & $112.47(15)$ \\
\hline
\end{tabular}




\begin{tabular}{|c|c|}
\hline $\mathrm{C}(18)-\mathrm{C}(22)-\mathrm{C}(23)$ & $107.36(14)$ \\
\hline $\mathrm{C}(26)-\mathrm{C}(22)-\mathrm{H}(22)$ & 109.3 \\
\hline $\mathrm{C}(18)-\mathrm{C}(22)-\mathrm{H}(22)$ & 109.3 \\
\hline $\mathrm{C}(23)-\mathrm{C}(22)-\mathrm{H}(22)$ & 109.3 \\
\hline$C(19)-C(23)-C(22)$ & $110.50(15)$ \\
\hline$C(19)-C(23)-C(24)$ & $110.65(15)$ \\
\hline$C(22)-C(23)-C(24)$ & $107.49(14)$ \\
\hline $\mathrm{C}(19)-\mathrm{C}(23)-\mathrm{P}(2)$ & $107.47(12)$ \\
\hline $\mathrm{C}(22)-\mathrm{C}(23)-\mathrm{P}(2)$ & $111.08(12)$ \\
\hline $\mathrm{C}(24)-\mathrm{C}(23)-\mathrm{P}(2)$ & $109.67(12)$ \\
\hline $\mathrm{C}(28)-\mathrm{C}(24)-\mathrm{C}(20)$ & $109.53(15)$ \\
\hline$C(28)-C(24)-C(23)$ & $111.93(15)$ \\
\hline$C(20)-C(24)-C(23)$ & $107.34(14)$ \\
\hline $\mathrm{C}(28)-\mathrm{C}(24)-\mathrm{H}(24)$ & 109.3 \\
\hline $\mathrm{C}(20)-\mathrm{C}(24)-\mathrm{H}(24)$ & 109.3 \\
\hline $\mathrm{C}(23)-\mathrm{C}(24)-\mathrm{H}(24)$ & 109.3 \\
\hline$C(21)-C(25)-C(27)$ & $110.19(15)$ \\
\hline $\mathrm{C}(21)-\mathrm{C}(25)-\mathrm{H}(25 \mathrm{~A})$ & 109.6 \\
\hline $\mathrm{C}(27)-\mathrm{C}(25)-\mathrm{H}(25 \mathrm{~A})$ & 109.6 \\
\hline $\mathrm{C}(21)-\mathrm{C}(25)-\mathrm{H}(25 \mathrm{~B})$ & 109.6 \\
\hline $\mathrm{C}(27)-\mathrm{C}(25)-\mathrm{H}(25 \mathrm{~B})$ & 109.6 \\
\hline $\mathrm{H}(25 \mathrm{~A})-\mathrm{C}(25)-\mathrm{H}(25 \mathrm{~B})$ & 108.1 \\
\hline$C(27)-C(26)-C(22)$ & $109.98(15)$ \\
\hline $\mathrm{C}(27)-\mathrm{C}(26)-\mathrm{H}(26 \mathrm{~A})$ & 109.7 \\
\hline $\mathrm{C}(22)-\mathrm{C}(26)-\mathrm{H}(26 \mathrm{~A})$ & 109.7 \\
\hline $\mathrm{C}(27)-\mathrm{C}(26)-\mathrm{H}(26 \mathrm{~B})$ & 109.7 \\
\hline $\mathrm{C}(22)-\mathrm{C}(26)-\mathrm{H}(26 \mathrm{~B})$ & 109.7 \\
\hline $\mathrm{H}(26 \mathrm{~A})-\mathrm{C}(26)-\mathrm{H}(26 \mathrm{~B})$ & 108.2 \\
\hline $\mathrm{C}(25)-\mathrm{C}(27)-\mathrm{C}(28)$ & $108.77(15)$ \\
\hline$C(25)-C(27)-C(26)$ & $108.53(15)$ \\
\hline $\mathrm{C}(28)-\mathrm{C}(27)-\mathrm{C}(26)$ & $110.25(15)$ \\
\hline $\mathrm{C}(25)-\mathrm{C}(27)-\mathrm{H}(27)$ & 109.8 \\
\hline $\mathrm{C}(28)-\mathrm{C}(27)-\mathrm{H}(27)$ & 109.8 \\
\hline $\mathrm{C}(26)-\mathrm{C}(27)-\mathrm{H}(27)$ & 109.8 \\
\hline $\mathrm{C}(27)-\mathrm{C}(28)-\mathrm{C}(24)$ & $110.01(15)$ \\
\hline $\mathrm{C}(27)-\mathrm{C}(28)-\mathrm{H}(28 \mathrm{~A})$ & 109.7 \\
\hline $\mathrm{C}(24)-\mathrm{C}(28)-\mathrm{H}(28 \mathrm{~A})$ & 109.7 \\
\hline $\mathrm{C}(27)-\mathrm{C}(28)-\mathrm{H}(28 \mathrm{~B})$ & 109.7 \\
\hline $\mathrm{C}(24)-\mathrm{C}(28)-\mathrm{H}(28 \mathrm{~B})$ & 109.7 \\
\hline $\mathrm{H}(28 \mathrm{~A})-\mathrm{C}(28)-\mathrm{H}(28 \mathrm{~B})$ & 108.2 \\
\hline$C(35)-C(29)-C(30)$ & $109.41(15)$ \\
\hline
\end{tabular}




\begin{tabular}{|c|c|}
\hline $\mathrm{C}(35)-\mathrm{C}(29)-\mathrm{H}(29 \mathrm{~A})$ & 109.8 \\
\hline $\mathrm{C}(30)-\mathrm{C}(29)-\mathrm{H}(29 \mathrm{~A})$ & 109.8 \\
\hline $\mathrm{C}(35)-\mathrm{C}(29)-\mathrm{H}(29 \mathrm{~B})$ & 109.8 \\
\hline $\mathrm{C}(30)-\mathrm{C}(29)-\mathrm{H}(29 \mathrm{~B})$ & 109.8 \\
\hline $\mathrm{H}(29 \mathrm{~A})-\mathrm{C}(29)-\mathrm{H}(29 \mathrm{~B})$ & 108.2 \\
\hline$C(31)-C(30)-C(29)$ & $110.28(16)$ \\
\hline$C(31)-C(30)-C(32)$ & $109.21(16)$ \\
\hline$C(29)-C(30)-C(32)$ & $108.94(16)$ \\
\hline $\mathrm{C}(31)-\mathrm{C}(30)-\mathrm{H}(30)$ & 109.5 \\
\hline $\mathrm{C}(29)-\mathrm{C}(30)-\mathrm{H}(30)$ & 109.5 \\
\hline $\mathrm{C}(32)-\mathrm{C}(30)-\mathrm{H}(30)$ & 109.5 \\
\hline $\mathrm{C}(34)-\mathrm{C}(31)-\mathrm{C}(30)$ & $109.23(15)$ \\
\hline $\mathrm{C}(34)-\mathrm{C}(31)-\mathrm{H}(31 \mathrm{~A})$ & 109.8 \\
\hline $\mathrm{C}(30)-\mathrm{C}(31)-\mathrm{H}(31 \mathrm{~A})$ & 109.8 \\
\hline $\mathrm{C}(34)-\mathrm{C}(31)-\mathrm{H}(31 \mathrm{~B})$ & 109.8 \\
\hline $\mathrm{C}(30)-\mathrm{C}(31)-\mathrm{H}(31 \mathrm{~B})$ & 109.8 \\
\hline $\mathrm{H}(31 \mathrm{~A})-\mathrm{C}(31)-\mathrm{H}(31 \mathrm{~B})$ & 108.3 \\
\hline$C(30)-C(32)-C(33)$ & $110.03(15)$ \\
\hline $\mathrm{C}(30)-\mathrm{C}(32)-\mathrm{H}(32 \mathrm{~A})$ & 109.7 \\
\hline $\mathrm{C}(33)-\mathrm{C}(32)-\mathrm{H}(32 \mathrm{~A})$ & 109.7 \\
\hline $\mathrm{C}(30)-\mathrm{C}(32)-\mathrm{H}(32 \mathrm{~B})$ & 109.7 \\
\hline $\mathrm{C}(33)-\mathrm{C}(32)-\mathrm{H}(32 \mathrm{~B})$ & 109.7 \\
\hline $\mathrm{H}(32 \mathrm{~A})-\mathrm{C}(32)-\mathrm{H}(32 \mathrm{~B})$ & 108.2 \\
\hline$C(32)-C(33)-C(37)$ & $110.65(15)$ \\
\hline$C(32)-C(33)-C(36)$ & $110.49(15)$ \\
\hline$C(37)-C(33)-C(36)$ & $107.42(14)$ \\
\hline $\mathrm{C}(32)-\mathrm{C}(33)-\mathrm{P}(3)$ & $107.65(12)$ \\
\hline $\mathrm{C}(37)-\mathrm{C}(33)-\mathrm{P}(3)$ & $110.13(12)$ \\
\hline $\mathrm{C}(36)-\mathrm{C}(33)-\mathrm{P}(3)$ & $110.53(12)$ \\
\hline$C(31)-C(34)-C(42)$ & $110.70(15)$ \\
\hline $\mathrm{C}(31)-\mathrm{C}(34)-\mathrm{C}(37)$ & $111.71(15)$ \\
\hline$C(42)-C(34)-C(37)$ & $108.91(15)$ \\
\hline $\mathrm{C}(31)-\mathrm{C}(34)-\mathrm{H}(34)$ & 108.5 \\
\hline $\mathrm{C}(42)-\mathrm{C}(34)-\mathrm{H}(34)$ & 108.5 \\
\hline $\mathrm{C}(37)-\mathrm{C}(34)-\mathrm{H}(34)$ & 108.5 \\
\hline$C(29)-C(35)-C(42)$ & $110.73(15)$ \\
\hline$C(29)-C(35)-C(36)$ & $111.18(15)$ \\
\hline$C(42)-C(35)-C(36)$ & $108.54(15)$ \\
\hline $\mathrm{C}(29)-\mathrm{C}(35)-\mathrm{H}(35)$ & 108.8 \\
\hline $\mathrm{C}(42)-\mathrm{C}(35)-\mathrm{H}(35)$ & 108.8 \\
\hline $\mathrm{C}(36)-\mathrm{C}(35)-\mathrm{H}(35)$ & 108.8 \\
\hline
\end{tabular}




\begin{tabular}{|c|c|}
\hline $\mathrm{C}(38)-\mathrm{C}(36)-\mathrm{C}(35)$ & $109.62(15)$ \\
\hline $\mathrm{C}(38)-\mathrm{C}(36)-\mathrm{C}(33)$ & $111.62(15)$ \\
\hline $\mathrm{C}(35)-\mathrm{C}(36)-\mathrm{C}(33)$ & $107.69(14)$ \\
\hline $\mathrm{C}(38)-\mathrm{C}(36)-\mathrm{H}(36)$ & 109.3 \\
\hline $\mathrm{C}(35)-\mathrm{C}(36)-\mathrm{H}(36)$ & 109.3 \\
\hline $\mathrm{C}(33)-\mathrm{C}(36)-\mathrm{H}(36)$ & 109.3 \\
\hline C(39)-C(37)-C(34) & $109.19(15)$ \\
\hline$C(39)-C(37)-C(33)$ & $112.52(15)$ \\
\hline $\mathrm{C}(34)-\mathrm{C}(37)-\mathrm{C}(33)$ & $107.24(14)$ \\
\hline C(39)-C(37)-H(37) & 109.3 \\
\hline C(34)-C(37)-H(37) & 109.3 \\
\hline $\mathrm{C}(33)-\mathrm{C}(37)-\mathrm{H}(37)$ & 109.3 \\
\hline $\mathrm{C}(40)-\mathrm{C}(38)-\mathrm{C}(36)$ & $110.27(16)$ \\
\hline $\mathrm{C}(40)-\mathrm{C}(38)-\mathrm{H}(38 \mathrm{~A})$ & 109.6 \\
\hline $\mathrm{C}(36)-\mathrm{C}(38)-\mathrm{H}(38 \mathrm{~A})$ & 109.6 \\
\hline $\mathrm{C}(40)-\mathrm{C}(38)-\mathrm{H}(38 \mathrm{~B})$ & 109.6 \\
\hline $\mathrm{C}(36)-\mathrm{C}(38)-\mathrm{H}(38 \mathrm{~B})$ & 109.6 \\
\hline $\mathrm{H}(38 \mathrm{~A})-\mathrm{C}(38)-\mathrm{H}(38 \mathrm{~B})$ & 108.1 \\
\hline C(40)-C(39)-C(37) & $109.99(15)$ \\
\hline $\mathrm{C}(40)-\mathrm{C}(39)-\mathrm{H}(39 \mathrm{~A})$ & 109.7 \\
\hline $\mathrm{C}(37)-\mathrm{C}(39)-\mathrm{H}(39 \mathrm{~A})$ & 109.7 \\
\hline $\mathrm{C}(40)-\mathrm{C}(39)-\mathrm{H}(39 \mathrm{~B})$ & 109.7 \\
\hline $\mathrm{C}(37)-\mathrm{C}(39)-\mathrm{H}(39 \mathrm{~B})$ & 109.7 \\
\hline H(39A)-C(39)-H(39B) & 108.2 \\
\hline $\mathrm{C}(38)-\mathrm{C}(40)-\mathrm{C}(39)$ & $110.46(16)$ \\
\hline $\mathrm{C}(38)-\mathrm{C}(40)-\mathrm{C}(41)$ & $108.27(16)$ \\
\hline C(39)-C(40)-C(41) & $108.92(17)$ \\
\hline $\mathrm{C}(38)-\mathrm{C}(40)-\mathrm{H}(40)$ & 109.7 \\
\hline $\mathrm{C}(39)-\mathrm{C}(40)-\mathrm{H}(40)$ & 109.7 \\
\hline $\mathrm{C}(41)-\mathrm{C}(40)-\mathrm{H}(40)$ & 109.7 \\
\hline $\mathrm{C}(42)-\mathrm{C}(41)-\mathrm{C}(40)$ & $110.05(15)$ \\
\hline $\mathrm{C}(42)-\mathrm{C}(41)-\mathrm{H}(41 \mathrm{~A})$ & 109.7 \\
\hline $\mathrm{C}(40)-\mathrm{C}(41)-\mathrm{H}(41 \mathrm{~A})$ & 109.7 \\
\hline $\mathrm{C}(42)-\mathrm{C}(41)-\mathrm{H}(41 \mathrm{~B})$ & 109.7 \\
\hline $\mathrm{C}(40)-\mathrm{C}(41)-\mathrm{H}(41 \mathrm{~B})$ & 109.7 \\
\hline $\mathrm{H}(41 \mathrm{~A})-\mathrm{C}(41)-\mathrm{H}(41 \mathrm{~B})$ & 108.2 \\
\hline $\mathrm{O}(5)-\mathrm{C}(42)-\mathrm{C}(41)$ & $109.76(15)$ \\
\hline $\mathrm{O}(5)-\mathrm{C}(42)-\mathrm{C}(34)$ & $110.11(15)$ \\
\hline $\mathrm{C}(41)-\mathrm{C}(42)-\mathrm{C}(34)$ & $111.23(15)$ \\
\hline $\mathrm{O}(5)-\mathrm{C}(42)-\mathrm{C}(35)$ & $107.42(14)$ \\
\hline$C(41)-C(42)-C(35)$ & $110.39(16)$ \\
\hline
\end{tabular}




\begin{tabular}{|c|c|}
\hline$C(34)-C(42)-C(35)$ & $107.84(15)$ \\
\hline $\mathrm{C}(48)-\mathrm{C}(43)-\mathrm{C}(44)$ & $109.03(16)$ \\
\hline $\mathrm{C}(48)-\mathrm{C}(43)-\mathrm{H}(43 \mathrm{~A})$ & 109.9 \\
\hline $\mathrm{C}(44)-\mathrm{C}(43)-\mathrm{H}(43 \mathrm{~A})$ & 109.9 \\
\hline $\mathrm{C}(48)-\mathrm{C}(43)-\mathrm{H}(43 \mathrm{~B})$ & 109.9 \\
\hline $\mathrm{C}(44)-\mathrm{C}(43)-\mathrm{H}(43 \mathrm{~B})$ & 109.9 \\
\hline $\mathrm{H}(43 \mathrm{~A})-\mathrm{C}(43)-\mathrm{H}(43 \mathrm{~B})$ & 108.3 \\
\hline$C(45)-C(44)-C(50)$ & $109.08(17)$ \\
\hline$C(45)-C(44)-C(43)$ & $109.15(17)$ \\
\hline $\mathrm{C}(50)-\mathrm{C}(44)-\mathrm{C}(43)$ & $110.31(17)$ \\
\hline $\mathrm{C}(45)-\mathrm{C}(44)-\mathrm{H}(44)$ & 109.4 \\
\hline $\mathrm{C}(50)-\mathrm{C}(44)-\mathrm{H}(44)$ & 109.4 \\
\hline $\mathrm{C}(43)-\mathrm{C}(44)-\mathrm{H}(44)$ & 109.4 \\
\hline$C(44)-C(45)-C(46)$ & $110.12(16)$ \\
\hline $\mathrm{C}(44)-\mathrm{C}(45)-\mathrm{H}(45 \mathrm{~A})$ & 109.6 \\
\hline $\mathrm{C}(46)-\mathrm{C}(45)-\mathrm{H}(45 \mathrm{~A})$ & 109.6 \\
\hline $\mathrm{C}(44)-\mathrm{C}(45)-\mathrm{H}(45 \mathrm{~B})$ & 109.6 \\
\hline $\mathrm{C}(46)-\mathrm{C}(45)-\mathrm{H}(45 \mathrm{~B})$ & 109.6 \\
\hline $\mathrm{H}(45 \mathrm{~A})-\mathrm{C}(45)-\mathrm{H}(45 \mathrm{~B})$ & 108.2 \\
\hline $\mathrm{C}(45)-\mathrm{C}(46)-\mathrm{C}(47)$ & $110.07(15)$ \\
\hline $\mathrm{C}(45)-\mathrm{C}(46)-\mathrm{C}(51)$ & $110.90(15)$ \\
\hline$C(47)-C(46)-C(51)$ & $107.00(15)$ \\
\hline $\mathrm{C}(45)-\mathrm{C}(46)-\mathrm{P}(4)$ & $108.00(13)$ \\
\hline $\mathrm{C}(47)-\mathrm{C}(46)-\mathrm{P}(4)$ & $110.80(13)$ \\
\hline $\mathrm{C}(51)-\mathrm{C}(46)-\mathrm{P}(4)$ & $110.10(12)$ \\
\hline C(49)-C(47)-C(48) & $109.51(16)$ \\
\hline C(49)-C(47)-C(46) & $111.89(16)$ \\
\hline $\mathrm{C}(48)-\mathrm{C}(47)-\mathrm{C}(46)$ & $107.92(15)$ \\
\hline $\mathrm{C}(49)-\mathrm{C}(47)-\mathrm{H}(47)$ & 109.2 \\
\hline $\mathrm{C}(48)-\mathrm{C}(47)-\mathrm{H}(47)$ & 109.2 \\
\hline $\mathrm{C}(46)-\mathrm{C}(47)-\mathrm{H}(47)$ & 109.2 \\
\hline$C(43)-C(48)-C(53)$ & $110.58(16)$ \\
\hline $\mathrm{C}(43)-\mathrm{C}(48)-\mathrm{C}(47)$ & $111.48(16)$ \\
\hline $\mathrm{C}(53)-\mathrm{C}(48)-\mathrm{C}(47)$ & $109.03(15)$ \\
\hline $\mathrm{C}(43)-\mathrm{C}(48)-\mathrm{H}(48)$ & 108.6 \\
\hline $\mathrm{C}(53)-\mathrm{C}(48)-\mathrm{H}(48)$ & 108.6 \\
\hline $\mathrm{C}(47)-\mathrm{C}(48)-\mathrm{H}(48)$ & 108.6 \\
\hline C(55)-C(49)-C(47) & $109.89(16)$ \\
\hline $\mathrm{C}(55)-\mathrm{C}(49)-\mathrm{H}(49 \mathrm{~A})$ & 109.7 \\
\hline $\mathrm{C}(47)-\mathrm{C}(49)-\mathrm{H}(49 \mathrm{~A})$ & 109.7 \\
\hline $\mathrm{C}(55)-\mathrm{C}(49)-\mathrm{H}(49 \mathrm{~B})$ & 109.7 \\
\hline
\end{tabular}




\begin{tabular}{|c|c|}
\hline $\mathrm{C}(47)-\mathrm{C}(49)-\mathrm{H}(49 \mathrm{~B})$ & 109.7 \\
\hline H(49A)-C(49)-H(49B) & 108.2 \\
\hline$C(52)-C(50)-C(44)$ & $109.53(16)$ \\
\hline $\mathrm{C}(52)-\mathrm{C}(50)-\mathrm{H}(50 \mathrm{~A})$ & 109.8 \\
\hline $\mathrm{C}(44)-\mathrm{C}(50)-\mathrm{H}(50 \mathrm{~A})$ & 109.8 \\
\hline $\mathrm{C}(52)-\mathrm{C}(50)-\mathrm{H}(50 \mathrm{~B})$ & 109.8 \\
\hline $\mathrm{C}(44)-\mathrm{C}(50)-\mathrm{H}(50 \mathrm{~B})$ & 109.8 \\
\hline $\mathrm{H}(50 \mathrm{~A})-\mathrm{C}(50)-\mathrm{H}(50 \mathrm{~B})$ & 108.2 \\
\hline$C(56)-C(51)-C(52)$ & $108.99(16)$ \\
\hline$C(56)-C(51)-C(46)$ & $112.63(16)$ \\
\hline$C(52)-C(51)-C(46)$ & $107.69(15)$ \\
\hline $\mathrm{C}(56)-\mathrm{C}(51)-\mathrm{H}(51)$ & 109.2 \\
\hline $\mathrm{C}(52)-\mathrm{C}(51)-\mathrm{H}(51)$ & 109.2 \\
\hline $\mathrm{C}(46)-\mathrm{C}(51)-\mathrm{H}(51)$ & 109.2 \\
\hline $\mathrm{C}(50)-\mathrm{C}(52)-\mathrm{C}(53)$ & $110.59(16)$ \\
\hline$C(50)-C(52)-C(51)$ & $111.07(16)$ \\
\hline$C(53)-C(52)-C(51)$ & $109.13(15)$ \\
\hline $\mathrm{C}(50)-\mathrm{C}(52)-\mathrm{H}(52)$ & 108.7 \\
\hline $\mathrm{C}(53)-\mathrm{C}(52)-\mathrm{H}(52)$ & 108.7 \\
\hline $\mathrm{C}(51)-\mathrm{C}(52)-\mathrm{H}(52)$ & 108.7 \\
\hline $\mathrm{O}(7)-\mathrm{C}(53)-\mathrm{C}(54)$ & $110.36(16)$ \\
\hline $\mathrm{O}(7)-\mathrm{C}(53)-\mathrm{C}(48)$ & $107.12(15)$ \\
\hline$C(54)-C(53)-C(48)$ & $110.70(16)$ \\
\hline $\mathrm{O}(7)-\mathrm{C}(53)-\mathrm{C}(52)$ & $110.38(15)$ \\
\hline$C(54)-C(53)-C(52)$ & $110.68(16)$ \\
\hline $\mathrm{C}(48)-\mathrm{C}(53)-\mathrm{C}(52)$ & $107.50(15)$ \\
\hline$C(53)-C(54)-C(55)$ & $109.77(16)$ \\
\hline $\mathrm{C}(53)-\mathrm{C}(54)-\mathrm{H}(54 \mathrm{~A})$ & 109.7 \\
\hline $\mathrm{C}(55)-\mathrm{C}(54)-\mathrm{H}(54 \mathrm{~A})$ & 109.7 \\
\hline $\mathrm{C}(53)-\mathrm{C}(54)-\mathrm{H}(54 \mathrm{~B})$ & 109.7 \\
\hline $\mathrm{C}(55)-\mathrm{C}(54)-\mathrm{H}(54 \mathrm{~B})$ & 109.7 \\
\hline $\mathrm{H}(54 \mathrm{~A})-\mathrm{C}(54)-\mathrm{H}(54 \mathrm{~B})$ & 108.2 \\
\hline$C(54)-C(55)-C(56)$ & $109.14(17)$ \\
\hline$C(54)-C(55)-C(49)$ & $108.93(17)$ \\
\hline$C(56)-C(55)-C(49)$ & $110.00(17)$ \\
\hline $\mathrm{C}(54)-\mathrm{C}(55)-\mathrm{H}(55)$ & 109.6 \\
\hline $\mathrm{C}(56)-\mathrm{C}(55)-\mathrm{H}(55)$ & 109.6 \\
\hline $\mathrm{C}(49)-\mathrm{C}(55)-\mathrm{H}(55)$ & 109.6 \\
\hline$C(55)-C(56)-C(51)$ & $109.84(16)$ \\
\hline $\mathrm{C}(55)-\mathrm{C}(56)-\mathrm{H}(56 \mathrm{~A})$ & 109.7 \\
\hline $\mathrm{C}(51)-\mathrm{C}(56)-\mathrm{H}(56 \mathrm{~A})$ & 109.7 \\
\hline
\end{tabular}


$\mathrm{C}(55)-\mathrm{C}(56)-\mathrm{H}(56 \mathrm{~B})$

109.7

$\mathrm{C}(51)-\mathrm{C}(56)-\mathrm{H}(56 \mathrm{~B})$

109.7

$\mathrm{H}(56 \mathrm{~A})-\mathrm{C}(56)-\mathrm{H}(56 \mathrm{~B})$

108.2 
(40) Tribenzyl(9-hydroxydiaman-4-yl) phosphonium bromide

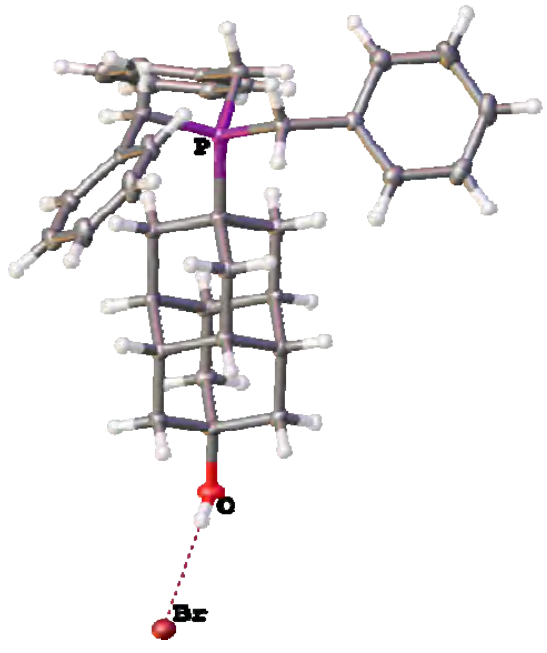

Ellipsoids are drawn at $50 \%$ probability.

Experimental. A single colourless prism-shaped crystal(Compound 40) was selected and mounted on a mylar loop oil on a Mo diffractometer. The crystal $(0.25 \times$ $0.15 \times 0.10 \mathrm{~mm}^{3}$ )was kept at $T=100 \mathrm{~K}$ during data collection. Using Olex2 (Dolomanov et al., 2009), the structure was solved with the ShelXS (Sheldrick, 2013) structure solution program, using the Direct Methods solution method. The model was refined with version 2013-3 of XL (Sheldrick, 2008) using Least Squares minimisation.

Crystal Data. $\mathrm{C}_{35} \mathrm{H}_{40} \mathrm{BrOP}, M_{r}=587.55$, monoclinic, $\mathrm{P} 2{ }_{1} / \mathrm{c}$ (No. 14), $a=10.3888(5) \AA, b=14.2107(7) \AA, c=$ 19.5603(10) $\AA, \quad \beta=100.963(2)^{\circ}, \alpha=\gamma=90^{\circ}, \quad V=$ 2835.0(2) $\AA^{3}, T=100 \mathrm{~K}, Z=4, Z^{\prime}=1, \mu\left(\mathrm{MoK}_{\alpha}\right)=1.534$, 58402 reflections measured, 6492 unique $\left(R_{\text {int }}=0.0598\right)$ which were used in all calculations. The final $w R_{2}$ was 0.0840 (all data) and $R_{1}$ was 0.0338 (I > 2(I)).

\begin{tabular}{|c|c|}
\hline $\begin{array}{l}\text { Compound } \\
\mathrm{N}^{\circ} \mathrm{CCDC}\end{array}$ & $\begin{array}{l}\text { Compound4C } \\
1054569\end{array}$ \\
\hline Formula & $\mathrm{C}_{35} \mathrm{H}_{40} \mathrm{BrOP}$ \\
\hline$D_{\text {calc. }} / \mathrm{g} \mathrm{cm}^{-3}$ & 1.377 \\
\hline$\mu / \mathrm{mm}^{-1}$ & 1.534 \\
\hline Formula Weight & 587.55 \\
\hline Colour & colourless \\
\hline Shape & prism \\
\hline Max Size/mm & 0.25 \\
\hline Mid Size/mm & 0.15 \\
\hline Min Size/mm & 0.10 \\
\hline$T / \mathrm{K}$ & 100 \\
\hline Crystal System & monoclinic \\
\hline Space Group & $\mathrm{P} 2_{1} / \mathrm{c}$ \\
\hline$a / \AA ̊$ & $10.3888(5)$ \\
\hline$b / \AA ̊$ & $14.2107(7)$ \\
\hline$c / \AA \AA$ & $19.5603(10)$ \\
\hline$\alpha /^{\circ}$ & 90 \\
\hline$\beta /^{\circ}$ & $100.963(2)$ \\
\hline$\gamma /{ }^{\circ}$ & 90 \\
\hline $\mathrm{V} / \AA^{3}$ & $2835.0(2)$ \\
\hline$Z$ & 4 \\
\hline$Z^{\prime}$ & 1 \\
\hline$\Theta_{\min } /^{\circ}$ & 2.867 \\
\hline$\Theta_{\max } /^{\circ}$ & 27.516 \\
\hline Measured Refl. & 58402 \\
\hline Independent Refl. & 6492 \\
\hline Reflections Used & 5141 \\
\hline$R_{\text {int }}$ & 0.0598 \\
\hline Parameters & 344 \\
\hline Restraints & 0 \\
\hline Largest Peak & 0.538 \\
\hline Deepest Hole & -0.455 \\
\hline GooF & 1.016 \\
\hline$w R_{2}$ (all data) & 0.0840 \\
\hline$w R_{2}$ & 0.0755 \\
\hline$R_{1}$ (all data) & 0.0534 \\
\hline$R_{1}$ & 0.0338 \\
\hline
\end{tabular}


Experimental Extended. A colourless prism-shaped crystal with dimensions $0.25 \times 0.15 \times 0.10 \mathrm{~mm}^{3}$ was mounted on a mylar loop with grease. Data were collected using $\mathrm{MoK}_{\alpha}$ radiation (X-ray tube, $50 \mathrm{kV}$, $32 \mathrm{~mA}$ ). The total number of runs and images was based on the strategy calculation from the program APEX2. Cell parameters were retrieved using the SAINT (V8.34A, 2013) software and refined using SAINT (V8.34A, 2013) on 9846 reflections, 18 of the observed reflections. Data reduction was performed using the SAINT (V8.34A, 2013) software which corrects for Lorentz polarisation. The final completeness is 99.70 out to 27.516 in $\Theta$. The absorption coefficient (MU) of this material is 1.534 and the minimum and maximum transmissions are 0.6331 and 0.7456 . The structure was solved in the space group P2 1 /c (\# 14) by Direct Methods using the ShelXS-2013 (Sheldrick, 2015) structure solution program and refined by Least Squares using version 2013-3 of XL (Sheldrick, 2008). All non-hydrogen atoms were refined anisotropically. Hydrogen atom positions were calculated geometrically and refined using the riding model.

Table S20: Fractional Atomic Coordinates $\left(\times 10^{4}\right)$ and Equivalent Isotropic Displacement Parameters $\left(\AA^{2} \times 10^{3}\right)$ for Compound 40. $U_{e q}$ is defined as $1 / 3$ of of the trace of the orthogonalised $U_{i j}$.

\begin{tabular}{|c|c|c|c|c|}
\hline Atom & $\mathrm{x}$ & $\mathrm{y}$ & $\mathrm{z}$ & $U_{e q}$ \\
\hline $\mathrm{Br}$ & $4721.4(2)$ & $8473.1(2)$ & $6307.5(2)$ & $20.87(7)$ \\
\hline $\mathrm{P}$ & $3800.3(5)$ & $1294.2(4)$ & $6089.6(3)$ & $11.69(11)$ \\
\hline 0 & $2400.2(15)$ & $6764.4(10)$ & $5831.9(8)$ & $19.8(3)$ \\
\hline C29 & $5153(2)$ & 973.7(14) & 5663.3(10) & $13.9(4)$ \\
\hline C15 & $4250(2)$ & $872.7(15)$ & $6982(1)$ & $14.2(4)$ \\
\hline C7 & $3566(2)$ & $2927.2(14)$ & 5308.3(10) & $13.0(4)$ \\
\hline C30 & $6484(2)$ & $1386.2(14)$ & 5965.3(10) & $14.0(4)$ \\
\hline C1 & $3492.1(19)$ & $2566.5(14)$ & $6046(1)$ & $11.6(4)$ \\
\hline C6 & $4528.6(19)$ & $3086.7(14)$ & $6593.5(10)$ & $13.1(4)$ \\
\hline C23 & $1598(2)$ & 892.3(14) & 4996.2(11) & $15.7(4)$ \\
\hline C16 & 3191.4(19) & 789.7(14) & $7416.4(10)$ & $13.4(4)$ \\
\hline $\mathrm{C} 24$ & $300(2)$ & $1169.9(15)$ & $4959.9(12)$ & $19.3(5)$ \\
\hline C22 & 2392(2) & $588.0(15)$ & 5693.6(11) & $16.3(4)$ \\
\hline C8 & $3314(2)$ & $3989.4(14)$ & $5275.3(10)$ & $12.4(4)$ \\
\hline C5 & $4258.4(19)$ & $4148.3(14)$ & $6543(1)$ & $11.8(4)$ \\
\hline $\mathrm{C} 2$ & 2118.0(19) & $2778.0(14)$ & $6202.8(11)$ & $13.8(4)$ \\
\hline $\mathrm{C} 25$ & $-454(2)$ & $1471.9(15)$ & $4336.0(13)$ & $24.2(5)$ \\
\hline $\mathrm{C} 4$ & $2885(2)$ & $4370.8(14)$ & $6691.2(10)$ & $13.6(4)$ \\
\hline C17 & 2998(2) & $1498.0(16)$ & $7880.2(11)$ & $18.8(4)$ \\
\hline C13 & 4339.3(19) & $4514.4(14)$ & $5812.7(10)$ & $12.4(4)$ \\
\hline C12 & $4076(2)$ & $5577.8(14)$ & $5765.0(11)$ & $14.9(4)$ \\
\hline C9 & 1939(2) & $4206.2(14)$ & $5420.3(10)$ & $14.0(4)$ \\
\hline $\mathrm{C} 21$ & $2468(2)$ & $-39.1(16)$ & 7398.6(11) & $20.6(5)$ \\
\hline C3 & $1866.7(19)$ & $3839.5(14)$ & $6154.7(10)$ & $13.3(4)$ \\
\hline C14 & $2620(2)$ & $5434.1(14)$ & $6629.9(10)$ & $14.4(4)$ \\
\hline C35 & $7017(2)$ & $2098.6(15)$ & $5615.6(11)$ & $17.4(4)$ \\
\hline C18 & 2094(2) & $1378.8(17)$ & 8314.7(11) & $23.9(5)$ \\
\hline C26 & $82(2)$ & $1494.2(16)$ & $3740.3(12)$ & $26.7(5)$ \\
\hline C31 & $7221(2)$ & $1043.6(16)$ & $6584.5(11)$ & $19.8(5)$ \\
\hline C19 & $1387(2)$ & 550.8(19) & $8294.4(12)$ & $26.5(5)$ \\
\hline C10 & 1691(2) & $5267.0(14)$ & $5371.3(11)$ & $15.7(4)$ \\
\hline C33 & $8914(2)$ & 2174.7(19) & $6529.3(12)$ & $26.5(5)$ \\
\hline C11 & $2710(2)$ & $5784.9(14)$ & 5903.5(11) & $14.8(4)$ \\
\hline C28 & $2116(2)$ & 896.5(16) & $4390.1(11)$ & $20.9(5)$ \\
\hline C34 & $8219(2)$ & 2491.3(17) & $5897.4(12)$ & $22.8(5)$ \\
\hline $\mathrm{C} 27$ & $1356(3)$ & $1197.0(17)$ & $3763.8(12)$ & $26.6(5)$ \\
\hline C32 & $8423(2)$ & $1441.0(19)$ & $6865.8(12)$ & $26.2(5)$ \\
\hline C20 & $1570(2)$ & $-153.9(19)$ & $7835.6(12)$ & $28.2(5)$ \\
\hline
\end{tabular}


Table S21: Anisotropic Displacement Parameters $\left(\times 10^{4}\right)$ Compound 40. The anisotropic displacement factor exponent takes the form: $-2 \pi^{2}\left[a^{* 2} \times U_{11}+\ldots 2 h k a^{*} \times b^{*} \times U_{12}\right]$

\begin{tabular}{|c|c|c|c|c|c|c|}
\hline Atom & $U_{11}$ & $U_{22}$ & $U_{33}$ & $U_{23}$ & $U_{13}$ & $U_{12}$ \\
\hline $\mathrm{Br}$ & $24.94(12)$ & $16.47(11)$ & $20.63(12)$ & $0.40(9)$ & $2.91(8)$ & $1.49(9)$ \\
\hline$P$ & $11.0(2)$ & $11.7(2)$ & $11.9(2)$ & $0.37(18)$ & $1.01(19)$ & $0.79(19)$ \\
\hline 0 & 21.8(8) & $11.4(7)$ & $24.3(8)$ & $-0.6(6)$ & $-0.8(7)$ & $1.9(6)$ \\
\hline C29 & $15.4(10)$ & $12.8(10)$ & $13.6(10)$ & $-1.1(8)$ & $3.6(8)$ & $2.6(8)$ \\
\hline C15 & $12(1)$ & $16(1)$ & $13.9(10)$ & $2.3(8)$ & $1.2(8)$ & $1.6(8)$ \\
\hline C7 & $14.4(10)$ & $13.4(10)$ & $11.2(9)$ & $-0.5(7)$ & $2.1(8)$ & $2.7(8)$ \\
\hline C30 & $13.2(10)$ & $15.3(10)$ & $14.3(9)$ & $-2.6(8)$ & $4.6(8)$ & $4.2(8)$ \\
\hline C1 & $10.7(10)$ & $12.1(9)$ & $11.8(9)$ & $0.5(7)$ & $1.3(7)$ & $1.7(7)$ \\
\hline C6 & $11.8(10)$ & $15.6(10)$ & $11.6(9)$ & $0.0(8)$ & $1.3(8)$ & $0.1(8)$ \\
\hline $\mathrm{C} 23$ & $15.2(10)$ & $10.7(9)$ & $19.3(10)$ & $-3.0(8)$ & $-1.1(8)$ & $-1.2(8)$ \\
\hline C16 & $11.4(9)$ & $16.3(10)$ & $11.5(9)$ & $3.3(8)$ & $-0.3(8)$ & $1.9(8)$ \\
\hline C24 & $17.0(11)$ & 14.1(10) & $25.3(11)$ & $-4.6(9)$ & $0.8(9)$ & $-1.9(8)$ \\
\hline C22 & $14.9(10)$ & $12.8(10)$ & $20(1)$ & $0.5(8)$ & $0.2(8)$ & $-1.5(8)$ \\
\hline C8 & $15.8(10)$ & $11.7(9)$ & $9.7(9)$ & $0.6(7)$ & $2.5(8)$ & $2.0(8)$ \\
\hline C5 & $10.7(9)$ & $13.2(9)$ & $10.7(9)$ & $-1.3(7)$ & $0.2(7)$ & $0.1(7)$ \\
\hline C2 & $10.7(10)$ & $14.2(10)$ & $16.5(10)$ & $-0.4(8)$ & $2.9(8)$ & $-0.9(8)$ \\
\hline C25 & $17.9(11)$ & $15.0(11)$ & $35.6(13)$ & $-4.1(9)$ & $-5.3(9)$ & $2.4(9)$ \\
\hline C4 & $15.2(10)$ & $14.3(10)$ & $11.8(9)$ & $-1.8(8)$ & $4.0(8)$ & $0.2(8)$ \\
\hline C17 & $22.5(11)$ & 18.4(11) & $14.5(10)$ & $0.7(8)$ & $0.8(8)$ & $1.9(9)$ \\
\hline C13 & $10.8(9)$ & $12.7(9)$ & $14.2(10)$ & $0.3(8)$ & $3.8(8)$ & 1.1(7) \\
\hline C12 & $15.5(10)$ & $12.3(10)$ & 17.1(10) & $0.4(8)$ & $3.2(8)$ & $-1.5(8)$ \\
\hline C9 & 13.1(10) & 13.1(10) & $14(1)$ & $-2.2(8)$ & $-1.7(8)$ & $1.6(8)$ \\
\hline C21 & $23.5(11)$ & $20.8(11)$ & 17.1(10) & $-1.2(9)$ & $3.3(9)$ & $-4.0(9)$ \\
\hline C3 & $8.6(9)$ & $14.8(10)$ & 17.1(10) & $-0.4(8)$ & $4.0(8)$ & $1.3(8)$ \\
\hline C14 & $13(1)$ & $15.4(10)$ & $14.7(10)$ & $-3.8(8)$ & $2.4(8)$ & $1.2(8)$ \\
\hline C35 & $17.9(11)$ & $17.9(11)$ & $17.9(10)$ & $4.0(8)$ & 7.4(8) & $5.5(8)$ \\
\hline C18 & $25.1(12)$ & $32.4(13)$ & $14.2(10)$ & $-1.0(9)$ & $3.9(9)$ & $9.6(10)$ \\
\hline C26 & $31.4(13)$ & 17.8(11) & $24.9(12)$ & $-0.4(9)$ & $-10.1(10)$ & $-0.9(10)$ \\
\hline C31 & $17.0(11)$ & 26.1(12) & $17.5(11)$ & $6.2(9)$ & $6.4(8)$ & $1.6(9)$ \\
\hline C19 & 17.1(11) & 47.1(15) & $15.7(11)$ & $3.5(10)$ & $4.5(9)$ & $-0.2(10)$ \\
\hline C10 & $15.3(10)$ & $13.6(10)$ & $16.7(10)$ & $-0.2(8)$ & $-0.9(8)$ & $3.2(8)$ \\
\hline C33 & $15.4(11)$ & $40.7(14)$ & $24.8(12)$ & $-3.7(10)$ & 7.1(9) & $-4.2(10)$ \\
\hline C11 & $16.5(10)$ & $10.4(9)$ & $16.8(10)$ & $-0.5(8)$ & $1.9(8)$ & $2.9(8)$ \\
\hline C28 & $18.2(11)$ & $21.5(11)$ & $21.3(11)$ & $-6.4(9)$ & $-0.6(9)$ & $0.5(9)$ \\
\hline C34 & $19.3(11)$ & $23.0(12)$ & $29.4(12)$ & $3.5(10)$ & $12.7(9)$ & $-0.3(9)$ \\
\hline C27 & $33.8(14)$ & $26.6(12)$ & $17.3(11)$ & $-4.8(9)$ & $-0.4(10)$ & $-2.6(10)$ \\
\hline C32 & $14.8(11)$ & $46.8(15)$ & $17.5(11)$ & $5.6(10)$ & $3.9(9)$ & $0.5(10)$ \\
\hline C20 & $25.6(12)$ & $37.0(14)$ & 22.1(12) & $1(1)$ & $4.9(10)$ & $-13.5(11)$ \\
\hline
\end{tabular}

Table S22: Bond Lengths in Å for Compound 40.

\begin{tabular}{|c|c|c|}
\hline Atom & Atom & Length/Å \\
\hline $\mathrm{P}$ & C29 & $1.823(2)$ \\
\hline$P$ & C15 & $1.820(2)$ \\
\hline $\mathrm{P}$ & $\mathrm{C} 1$ & $1.835(2)$ \\
\hline $\mathrm{P}$ & $\mathrm{C} 22$ & $1.821(2)$ \\
\hline 0 & C11 & $1.429(2)$ \\
\hline $\mathrm{C} 29$ & $\mathrm{C} 30$ & $1.514(3)$ \\
\hline C15 & C16 & $1.517(3)$ \\
\hline $\mathrm{C} 7$ & $\mathrm{C} 1$ & $1.547(3)$ \\
\hline C7 & C8 & $1.531(3)$ \\
\hline C30 & C35 & $1.394(3)$ \\
\hline C30 & C31 & $1.392(3)$ \\
\hline $\mathrm{C} 1$ & C6 & $1.554(3)$ \\
\hline $\mathrm{C} 1$ & $\mathrm{C} 2$ & $1.546(3)$ \\
\hline C6 & $\mathrm{C} 5$ & $1.534(3)$ \\
\hline $\mathrm{C} 23$ & $\mathrm{C} 24$ & $1.393(3)$ \\
\hline $\mathrm{C} 23$ & $\mathrm{C} 22$ & $1.516(3)$ \\
\hline
\end{tabular}

\begin{tabular}{lll}
\hline Atom & Atom & Length/§ \\
\hline C23 & C28 & $1.392(3)$ \\
C16 & C17 & $1.395(3)$ \\
C16 & C21 & $1.394(3)$ \\
C24 & C25 & $1.387(3)$ \\
C8 & C13 & $1.539(3)$ \\
C8 & C9 & $1.539(3)$ \\
C5 & C4 & $1.542(3)$ \\
C5 & C13 & $1.537(3)$ \\
C2 & C3 & $1.531(3)$ \\
C25 & C26 & $1.384(4)$ \\
C4 & C3 & $1.539(3)$ \\
C4 & C14 & $1.537(3)$ \\
C17 & C18 & $1.392(3)$ \\
C13 & C12 & $1.535(3)$ \\
C12 & C11 & $1.524(3)$ \\
C9 & C3 & $1.543(3)$
\end{tabular}




\begin{tabular}{|c|c|c|}
\hline Atom & Atom & Length/Å \\
\hline $\mathrm{C} 9$ & C10 & $1.529(3)$ \\
\hline C21 & $\mathrm{C} 20$ & $1.390(3)$ \\
\hline C14 & C11 & $1.525(3)$ \\
\hline C35 & C34 & $1.383(3)$ \\
\hline C18 & C19 & $1.384(4)$ \\
\hline C26 & $\mathrm{C} 27$ & $1.382(4)$ \\
\hline
\end{tabular}

\begin{tabular}{|c|c|c|}
\hline Atom & Atom & Length/Å \\
\hline$\overline{\mathrm{C} 31}$ & C32 & $1.386(3)$ \\
\hline C19 & $\mathrm{C} 20$ & $1.382(4)$ \\
\hline C10 & C11 & $1.525(3)$ \\
\hline C33 & C34 & $1.383(3)$ \\
\hline C33 & $\mathrm{C} 32$ & $1.381(3)$ \\
\hline C28 & $\mathrm{C} 27$ & $1.392(3)$ \\
\hline
\end{tabular}

Table S23: Bond Angles in ${ }^{\circ}$ for Compound 40.

\begin{tabular}{|c|c|c|c|}
\hline Atom & Atom & Atom & Angle/ ${ }^{\circ}$ \\
\hline $\mathrm{C} 29$ & $\mathrm{P}$ & C1 & $111.58(9)$ \\
\hline C15 & $\mathrm{P}$ & $\mathrm{C} 29$ & $106.09(9)$ \\
\hline C15 & $\mathrm{P}$ & $\mathrm{C} 1$ & $112.29(9)$ \\
\hline C15 & $\mathrm{P}$ & $\mathrm{C} 22$ & $105.38(10)$ \\
\hline $\mathrm{C} 22$ & $\mathrm{P}$ & $\mathrm{C} 29$ & $107.15(10)$ \\
\hline $\mathrm{C} 22$ & $\mathrm{P}$ & $\mathrm{C} 1$ & $113.83(9)$ \\
\hline C30 & $\mathrm{C} 29$ & $\mathrm{P}$ & $116.80(14)$ \\
\hline C16 & C15 & $\mathrm{P}$ & $118.81(14)$ \\
\hline C8 & $\mathrm{C} 7$ & $\mathrm{C} 1$ & $109.12(16)$ \\
\hline C35 & C30 & $\mathrm{C} 29$ & $120.55(18)$ \\
\hline C31 & C30 & $\mathrm{C} 29$ & $120.95(19)$ \\
\hline C31 & C30 & C35 & $118.5(2)$ \\
\hline $\mathrm{C} 7$ & $\mathrm{C} 1$ & $\mathrm{P}$ & $109.30(13)$ \\
\hline $\mathrm{C} 7$ & $\mathrm{C} 1$ & C6 & $109.53(16)$ \\
\hline C6 & $\mathrm{C} 1$ & $\mathrm{P}$ & $110.17(13)$ \\
\hline $\mathrm{C} 2$ & $\mathrm{C} 1$ & $\mathrm{P}$ & $110.00(13)$ \\
\hline $\mathrm{C} 2$ & $\mathrm{C} 1$ & $\mathrm{C} 7$ & $109.41(16)$ \\
\hline $\mathrm{C} 2$ & $\mathrm{C} 1$ & $\mathrm{C} 6$ & $108.42(16)$ \\
\hline $\mathrm{C} 5$ & C6 & $\mathrm{C} 1$ & $109.21(16)$ \\
\hline $\mathrm{C} 24$ & $\mathrm{C} 23$ & $\mathrm{C} 22$ & $118.79(19)$ \\
\hline C28 & $\mathrm{C} 23$ & $\mathrm{C} 24$ & 118.8(2) \\
\hline C28 & $\mathrm{C} 23$ & $\mathrm{C} 22$ & $122.38(19)$ \\
\hline C17 & C16 & C15 & $121.17(19)$ \\
\hline $\mathrm{C} 21$ & C16 & C15 & $119.77(19)$ \\
\hline C21 & C16 & $\mathrm{C} 17$ & $118.84(19)$ \\
\hline $\mathrm{C} 25$ & $\mathrm{C} 24$ & $\mathrm{C} 23$ & $120.8(2)$ \\
\hline C23 & $\mathrm{C} 22$ & $\mathrm{P}$ & $118.73(15)$ \\
\hline C7 & $\mathrm{C} 8$ & C13 & $111.01(16)$ \\
\hline $\mathrm{C} 7$ & $\mathrm{C} 8$ & $\mathrm{C} 9$ & $110.28(16)$ \\
\hline C9 & $\mathrm{C} 8$ & C13 & $108.83(16)$ \\
\hline C6 & $\mathrm{C} 5$ & $\mathrm{C} 4$ & $110.74(16)$ \\
\hline C6 & $\mathrm{C} 5$ & C13 & $110.54(16)$ \\
\hline C13 & $\mathrm{C} 5$ & $\mathrm{C} 4$ & $108.75(16)$ \\
\hline C3 & $\mathrm{C} 2$ & $\mathrm{C} 1$ & $109.43(16)$ \\
\hline C26 & $\mathrm{C} 25$ & $\mathrm{C} 24$ & $119.8(2)$ \\
\hline C3 & $\mathrm{C} 4$ & $\mathrm{C} 5$ & $108.17(16)$ \\
\hline C14 & $\mathrm{C} 4$ & $\mathrm{C} 5$ & $110.23(17)$ \\
\hline C14 & $\mathrm{C} 4$ & $\mathrm{C} 3$ & $109.83(16)$ \\
\hline C18 & $\mathrm{C} 17$ & C16 & $120.4(2)$ \\
\hline C5 & C13 & C8 & $108.51(16)$ \\
\hline C12 & C13 & C8 & $110.09(16)$ \\
\hline C12 & C13 & $\mathrm{C} 5$ & $110.43(16)$ \\
\hline C11 & $\mathrm{C} 12$ & C13 & $109.90(17)$ \\
\hline C8 & $\mathrm{C} 9$ & $\mathrm{C} 3$ & $108.38(16)$ \\
\hline C10 & C9 & C8 & $109.71(17)$ \\
\hline C10 & C9 & $\mathrm{C} 3$ & $110.71(16)$ \\
\hline $\mathrm{C} 20$ & $\mathrm{C} 21$ & C16 & $120.4(2)$ \\
\hline $\mathrm{C} 2$ & $\mathrm{C} 3$ & $\mathrm{C} 4$ & $110.76(16)$ \\
\hline $\mathrm{C} 2$ & $\mathrm{C} 3$ & $\mathrm{C} 9$ & $110.61(16)$ \\
\hline $\mathrm{C} 4$ & $\mathrm{C} 3$ & $\mathrm{C} 9$ & $108.66(16)$ \\
\hline
\end{tabular}

\begin{tabular}{llll}
\hline Atom & Atom & Atom & \multicolumn{1}{c}{ Angle $^{\circ}$} \\
\hline C11 & C14 & C4 & $110.62(16)$ \\
C34 & C35 & C30 & $120.7(2)$ \\
C19 & C18 & C17 & $120.2(2)$ \\
C27 & C26 & C25 & $120.1(2)$ \\
C32 & C31 & C30 & $120.5(2)$ \\
C20 & C19 & C18 & $119.8(2)$ \\
C11 & C10 & C9 & $110.22(16)$ \\
C32 & C33 & C34 & $119.6(2)$ \\
O & C11 & C12 & $111.76(17)$ \\
O & C11 & C14 & $110.91(16)$ \\
O & C11 & C10 & $106.79(16)$ \\
C12 & C11 & C14 & $109.08(17)$ \\
C12 & C11 & C10 & $109.55(17)$ \\
C14 & C11 & C10 & $108.68(17)$ \\
C23 & C28 & C27 & $120.3(2)$ \\
C33 & C34 & C35 & $120.2(2)$ \\
C26 & C27 & C28 & $120.1(2)$ \\
C33 & C32 & C31 & $120.3(2)$ \\
C19 & C20 & C21 & $120.4(2)$
\end{tabular}


Table S24: Hydrogen Fractional Atomic Coordinates $\left(\times 10^{4}\right)$ and Equivalent Isotropic Displacement Parameters $\left(\AA^{2} \times 10^{3}\right)$ for Compound 40. $U_{e q}$ is defined as $1 / 3$ of of the trace of the orthogonalised $U_{i j \text {. }}$

\begin{tabular}{|c|c|c|c|c|}
\hline Atom & & & & $U_{e q}$ \\
\hline$\overline{\mathrm{H}}$ & 3065 & 7083 & 6002 & 30 \\
\hline H29A & 5234 & 279 & 5673 & 17 \\
\hline H29B & 4919 & 1166 & 5169 & 17 \\
\hline H15A & 4654 & 244 & 6968 & 17 \\
\hline H15B & 4937 & 1297 & 7232 & 17 \\
\hline H7A & 4443 & 2792 & 5203 & 16 \\
\hline H7B & 2901 & 2602 & 4958 & 16 \\
\hline H6A & 4483 & 2860 & 7067 & 16 \\
\hline H6B & 5419 & 2956 & 6506 & 16 \\
\hline H24 & -72 & 1152 & 5367 & 23 \\
\hline $\mathrm{H} 22 \mathrm{~A}$ & 2707 & -60 & 5639 & 20 \\
\hline $\mathrm{H} 22 \mathrm{~B}$ & 1787 & 557 & 6028 & 20 \\
\hline H8 & 3368 & 4221 & 4799 & 15 \\
\hline H5 & 4931 & 4480 & 6894 & 14 \\
\hline $\mathrm{H} 2 \mathrm{~A}$ & 1442 & 2445 & 5864 & 17 \\
\hline $\mathrm{H} 2 \mathrm{~B}$ & 2066 & 2552 & 6676 & 17 \\
\hline $\mathrm{H} 25$ & -1336 & 1663 & 4318 & 29 \\
\hline $\mathrm{H} 4$ & 2829 & 4154 & 7171 & 16 \\
\hline H17 & 3487 & 2066 & 7900 & 23 \\
\hline H13 & 5234 & 4386 & 5716 & 15 \\
\hline H12A & 4740 & 5911 & 6111 & 18 \\
\hline H12B & 4147 & 5807 & 5296 & 18 \\
\hline H9 & 1266 & 3876 & 5068 & 17 \\
\hline $\mathrm{H} 21$ & 2590 & -528 & 7086 & 25 \\
\hline H3 & 972 & 3969 & 6251 & 16 \\
\hline H14A & 1735 & 5568 & 6724 & 17 \\
\hline H14B & 3268 & 5772 & 6981 & 17 \\
\hline H35 & 6550 & 2316 & 5179 & 21 \\
\hline H18 & 1962 & 1867 & 8626 & 29 \\
\hline H26 & -427 & 1714 & 3315 & 32 \\
\hline H31 & 6897 & 533 & 6816 & 24 \\
\hline H19 & 777 & 467 & 8595 & 32 \\
\hline $\mathrm{H} 10 \mathrm{~A}$ & 1736 & 5489 & 4897 & 19 \\
\hline H10B & 802 & 5405 & 5459 & 19 \\
\hline H33 & 9725 & 2460 & 6731 & 32 \\
\hline H28 & 2991 & 693 & 4404 & 25 \\
\hline H34 & 8569 & 2980 & 5656 & 27 \\
\hline $\mathrm{H} 27$ & 1714 & 1198 & 3352 & 32 \\
\hline H32 & 8913 & 1208 & 7292 & 31 \\
\hline $\mathrm{H} 20$ & 1079 & -720 & 7819 & 34 \\
\hline
\end{tabular}




\section{(41) 1-Adamantyldiphenylphosphine oxide}

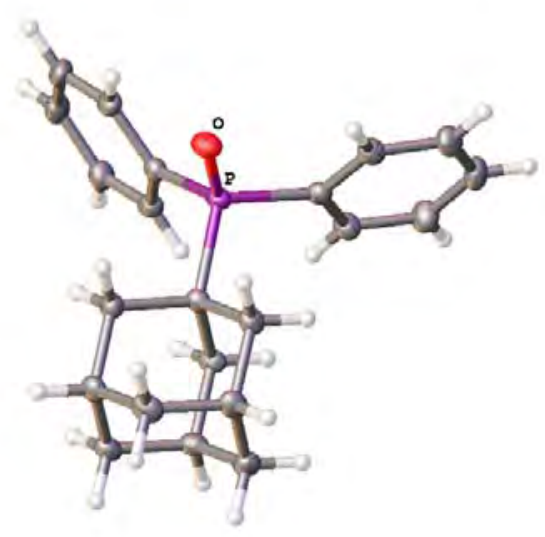

Ellipsoids are drawn at $50 \%$ probability.

Experimental. A single colourless prism-shaped crystals suitable crystal (Compound 41) was selected and mounted on a diffractometer. The crystal $(0.38 \times 0.20 \times 0.10)$ was kept at $T=115 \mathrm{~K}$ during data collection. Using Olex2 (Dolomanov et al., 2009), the structure was solved with the ShelXS-97 (Sheldrick, 2008) structure solution program, using the direct methods solution method. The model was refined with version of XL (Sheldrick, 2008) using Least Squares minimisation.

Crystal Data. $\mathrm{C}_{22} \mathrm{H}_{25} \mathrm{OP}, M_{r}=336.39$, monoclinic, $\mathrm{P} 2_{1} / \mathrm{n}$ (No. 14$), \quad \mathrm{a}=8.9308(2) \AA, \quad \mathrm{b}=12.0815(5) \AA, \quad \mathrm{c}=$ 16.2049(6) $\AA, \alpha=90.00^{\circ}, \beta=90.127(2)^{\circ}, \gamma=90.00^{\circ}, V=$ $1748.46(10) \AA^{3}, T=115 \mathrm{~K}, Z=4, Z^{\prime}=1, \mu\left(\mathrm{Mo} \mathrm{K}_{\alpha} \sim 1 \sim\right)=$ $0.163,9232$ reflections measured, 3931 unique $\left(R_{\text {int }}=\right.$ $0.0224)$ which were used in all calculations. The final $w R_{2}$ was 0.1010 (all data) and $R_{1}$ was $0.0409(>2 \sigma(\mathrm{I}))$.

\begin{tabular}{|c|c|}
\hline Compound & Compound 4 \\
\hline $\mathrm{N}^{\circ} \mathrm{CCDC}$ & 1054566 \\
\hline Formula & $\mathrm{C}_{22} \mathrm{H}_{25} \mathrm{OP}$ \\
\hline$D_{\text {calc. }} / \mathrm{g} \mathrm{cm}^{-3}$ & 1.278 \\
\hline$\mu / \mathrm{mm}^{-1}$ & 0.163 \\
\hline Formula Weight & 336.39 \\
\hline Colour & colourless \\
\hline Shape & prism \\
\hline Max Size/mm & 0.38 \\
\hline Mid Size/mm & 0.20 \\
\hline Min Size/mm & 0.10 \\
\hline$T / \mathrm{K}$ & 115 \\
\hline Crystal System & monoclinic \\
\hline Space Group & $\mathrm{P} 2_{1} / \mathrm{n}$ \\
\hline$a / \AA$ & $8.9308(2)$ \\
\hline$b / \AA ̊$ & $12.0815(5)$ \\
\hline$c / \AA \AA$ & $16.2049(6)$ \\
\hline$\alpha l^{\circ}$ & 90.00 \\
\hline$\left.\beta\right|^{\circ}$ & $90.127(2)$ \\
\hline$\gamma 1^{\circ}$ & 90.00 \\
\hline $\mathrm{V} / \AA^{3}$ & $1748.46(10)$ \\
\hline Z & 4 \\
\hline$Z^{\prime}$ & 1 \\
\hline$\left.\Theta_{\min }\right|^{\circ}$ & 2.84 \\
\hline$\Theta_{\max } /^{\circ}$ & 27.52 \\
\hline Measured Refl. & 9232 \\
\hline Independent Refl. & 3931 \\
\hline Reflections Used & 3433 \\
\hline$R_{\text {int }}$ & 0.0224 \\
\hline Parameters & 217 \\
\hline Restraints & 0 \\
\hline Largest Peak & 0.517 \\
\hline Deepest Hole & -0.330 \\
\hline GooF & 1.100 \\
\hline$w R_{2}$ (all data) & 0.1010 \\
\hline$w R_{2}$ & 0.0947 \\
\hline$R_{1}$ (all data) & 0.0498 \\
\hline$R_{1}$ & 0.0409 \\
\hline
\end{tabular}


Experimental Extended. A colourless prism-shaped crystal with dimensions $0.38 \times 0.20 \times 0.10 \mathrm{~mm}^{3}$ was mounted on a mylar loop with grease. Cell parameters were retrieved using the SCALEPACK (Otwinowski, 1997) software and refined using DENZO (Otwinowski, 1997). Data reduction was performed using the DENZO software (Otwinowski, 1997) which corrects for Lorentz polarization. The final completeness is 97.50 out to 27.52 in $Q$. No absoprtion correction was performed. The absorption coefficient $(\mathrm{m})$ of this material is 0.163 and the minimum and maximum transmissions are 0.9408 and 0.9839. The structure was solved by Direct Methods using the ShelXS-97 (Sheldrick, 2008) structure solution program and refined by Least Squares using version 2013-3 of XL (Sheldrick, 2008). The structure was solved in the space group $\mathrm{P} 2{ }_{1} / \mathrm{n}$ (\# 14). All non-hydrogen atoms were refined anisotropically. Hydrogen atom positions were calculated geometrically and refined using the riding model.

Table S25: Fractional Atomic Coordinates $\left(\times 10^{4}\right)$ and Equivalent Isotropic Displacement Parameters $\left(\AA^{2} \times 10^{3}\right)$ for Compound 41. $U_{e q}$ is defined as $1 / 3$ of the trace of the orthogonalised $U_{i j}$.

\begin{tabular}{lccll}
\hline Atom & $\mathbf{x}$ & $\mathbf{y}$ & $\mathbf{z}$ & $\boldsymbol{U}_{\text {eq }}$ \\
\hline C1 & $4356.5(16)$ & $6135.9(13)$ & $8189.7(9)$ & $17.4(3)$ \\
C2 & $5002.4(17)$ & $5819.6(14)$ & $9045(1)$ & $21.6(3)$ \\
C3 & $6720.0(18)$ & $5939.8(15)$ & $9039.4(10)$ & $24.7(3)$ \\
C4 & $7140.1(18)$ & $7140.9(15)$ & $8840.5(11)$ & $26.0(4)$ \\
C5 & $6508.4(17)$ & $7459.8(14)$ & $7996.1(10)$ & $22.4(3)$ \\
C6 & $4792.6(17)$ & $7340.6(13)$ & $7998.9(10)$ & $20.7(3)$ \\
C7 & $7380.6(18)$ & $5171.8(15)$ & $8379.8(11)$ & $26.6(4)$ \\
C8 & $6757.8(17)$ & $5497.0(14)$ & $7528.6(10)$ & $23.2(3)$ \\
C9 & $7176.5(18)$ & $6700.4(14)$ & $7337.8(10)$ & $23.7(3)$ \\
C10 & $5051.2(17)$ & $5365.3(13)$ & $7531(1)$ & $20.9(3)$ \\
C11 & $1515.6(16)$ & $6805.1(13)$ & $8976.3(9)$ & $18.9(3)$ \\
C12 & $948.4(18)$ & $6310.3(14)$ & $9689.8(10)$ & $22.6(3)$ \\
C13 & $244(2)$ & $6939.6(15)$ & $10295.4(10)$ & $27.3(4)$ \\
C14 & $126(2)$ & $8074.4(16)$ & $10194.4(10)$ & $29.8(4)$ \\
C15 & $708(2)$ & $8581.1(15)$ & $9494.4(11)$ & $28.9(4)$ \\
C16 & $1393.8(18)$ & $7951.8(14)$ & $8884.2(10)$ & $23.7(3)$ \\
C17 & $1506.9(16)$ & $6190.6(13)$ & $7224.4(9)$ & $18.5(3)$ \\
C18 & $1563.1(17)$ & $7212.4(13)$ & $6817.4(10)$ & $20.8(3)$ \\
C19 & $826.8(19)$ & $7353.8(15)$ & $6066.9(10)$ & $25.0(3)$ \\
C20 & $23(2)$ & $6487.1(16)$ & $5719.1(10)$ & $29.0(4)$ \\
C21 & $-25(2)$ & $5470.6(15)$ & $6112.5(10)$ & $28.4(4)$ \\
C22 & $709.5(18)$ & $5323.5(13)$ & $6859.3(10)$ & $23.2(3)$ \\
O & $2026.8(12)$ & $4695.0(9)$ & $8446.7(7)$ & $23.8(3)$ \\
P & $2336.3(4)$ & $5872.7(3)$ & $8222.1(2)$ & $17.67(11)$ \\
& & & &
\end{tabular}

Table S26: Anisotropic Displacement Parameters $\left(\times 10^{4}\right)$ Compound 41. The anisotropic displacement factor exponent takes the form: $-2 \pi^{2}\left[h^{2} a^{* 2} \times U_{11}+\ldots+2 h k a * \times b^{*} \times U_{12}\right]$

\begin{tabular}{lllrrrr}
\hline Atom & $\boldsymbol{U}_{\mathbf{1 1}}$ & $\boldsymbol{U}_{\mathbf{2 2}}$ & $\boldsymbol{U}_{\mathbf{3}}$ & $\boldsymbol{U}_{\mathbf{2 3}}$ & $\boldsymbol{U}_{\mathbf{1 3}}$ & $\boldsymbol{U}_{\mathbf{1 2}}$ \\
\hline $\mathrm{C} 1$ & $16.4(7)$ & $16.3(7)$ & $19.6(7)$ & $1.9(6)$ & $0.7(5)$ & $2.2(5)$ \\
$\mathrm{C} 2$ & $21.7(8)$ & $23.4(8)$ & $19.6(7)$ & $3.2(6)$ & $0.3(6)$ & $2.0(6)$ \\
C3 & $20.4(8)$ & $31.8(9)$ & $22.1(8)$ & $4.1(7)$ & $-2.7(6)$ & $3.4(6)$ \\
C4 & $19.8(8)$ & $30.2(9)$ & $27.9(8)$ & $-4.3(7)$ & $-1.4(6)$ & $-1.2(6)$ \\
C5 & $18.8(7)$ & $20.2(8)$ & $28.2(8)$ & $2.5(6)$ & $1.2(6)$ & $-2.1(6)$ \\
C6 & $18.3(7)$ & $17.0(8)$ & $26.7(8)$ & $3.0(6)$ & $1.4(6)$ & $1.3(6)$ \\
C7 & $20.8(8)$ & $25.4(9)$ & $33.7(9)$ & $5.6(7)$ & $0.9(7)$ & $6.8(6)$ \\
C8 & $20.0(7)$ & $24.2(8)$ & $25.3(8)$ & $-2.4(6)$ & $3.3(6)$ & $4.0(6)$ \\
C9 & $19.2(8)$ & $28.1(9)$ & $23.9(8)$ & $3.5(7)$ & $3.2(6)$ & $-1.4(6)$ \\
C10 & $21.6(8)$ & $19.6(8)$ & $21.5(7)$ & $-1.1(6)$ & $1.6(6)$ & $1.4(6)$ \\
C11 & $16.2(7)$ & $18.8(8)$ & $21.8(7)$ & $0.4(6)$ & $-0.8(5)$ & $0.7(6)$ \\
C12 & $22.2(8)$ & $23.5(8)$ & $22.0(8)$ & $4.5(6)$ & $-0.6(6)$ & $1.6(6)$ \\
C13 & $31.3(9)$ & $31.7(9)$ & $19.1(7)$ & $2.3(7)$ & $1.2(6)$ & $0.8(7)$ \\
C14 & $36.3(10)$ & $31.3(10)$ & $21.8(8)$ & $-6.0(7)$ & $1.9(7)$ & $3.8(7)$
\end{tabular}




\begin{tabular}{lllrrrr}
\hline Atom & $\boldsymbol{U}_{\mathbf{1 1}}$ & $\boldsymbol{U}_{\mathbf{2 2}}$ & $\boldsymbol{U}_{\mathbf{3 3}}$ & $\boldsymbol{U}_{23}$ & $\boldsymbol{U}_{13}$ & $\boldsymbol{U}_{\mathbf{1 2}}$ \\
\hline $\mathrm{C} 15$ & $36.1(9)$ & $20.9(8)$ & $29.8(9)$ & $-2.0(7)$ & $2.4(7)$ & $3.5(7)$ \\
$\mathrm{C} 16$ & $25.5(8)$ & $21.1(8)$ & $24.6(8)$ & $3.6(6)$ & $2.9(6)$ & $1.0(6)$ \\
$\mathrm{C} 17$ & $17.6(7)$ & $18.6(7)$ & $19.3(7)$ & $-0.8(6)$ & $2.5(5)$ & $1.7(6)$ \\
$\mathrm{C} 18$ & $19.2(7)$ & $19.6(8)$ & $23.7(8)$ & $-0.3(6)$ & $1.7(6)$ & $-0.5(6)$ \\
$\mathrm{C} 19$ & $28.3(8)$ & $25.9(9)$ & $20.8(8)$ & $1.8(6)$ & $2.4(6)$ & $0.9(7)$ \\
$\mathrm{C} 20$ & $31.1(9)$ & $37.5(10)$ & $18.5(8)$ & $-4.7(7)$ & $-0.8(6)$ & $-1.7(7)$ \\
$\mathrm{C} 21$ & $31.2(9)$ & $30.1(9)$ & $23.9(8)$ & $-10.3(7)$ & $3.0(7)$ & $-7.4(7)$ \\
$\mathrm{C} 22$ & $25.7(8)$ & $17.7(8)$ & $26.2(8)$ & $-3.3(6)$ & $5.4(6)$ & $-1.8(6)$ \\
O & $23.2(6)$ & $16.4(6)$ & $32.0(6)$ & $4.9(5)$ & $3.4(5)$ & $-0.1(4)$ \\
P & $16.93(19)$ & $14.3(2)$ & $21.7(2)$ & $2.24(15)$ & $1.67(14)$ & $0.16(14)$
\end{tabular}

Table S27: Bond Lengths in $\AA$ for Compound 41.

\begin{tabular}{lll}
\hline Atom & Atom & Length/A \\
\hline C1 & C2 & $1.548(2)$ \\
C1 & C6 & $1.538(2)$ \\
C1 & C10 & $1.547(2)$ \\
C1 & P & $1.8329(15)$ \\
C2 & C3 & $1.541(2)$ \\
C3 & C4 & $1.533(2)$ \\
C3 & C7 & $1.534(2)$ \\
C4 & C5 & $1.528(2)$ \\
C5 & C6 & $1.539(2)$ \\
C5 & C9 & $1.529(2)$ \\
C7 & C8 & $1.537(2)$ \\
C8 & C9 & $1.533(2)$ \\
C8 & C10 & $1.532(2)$ \\
C11 & C12 & $1.398(2)$
\end{tabular}

\begin{tabular}{lll}
\hline Atom & Atom & Length/ $\AA$ \\
\hline $\mathrm{C} 11$ & $\mathrm{C} 16$ & $1.398(2)$ \\
$\mathrm{C} 11$ & $\mathrm{P}$ & $1.8177(16)$ \\
$\mathrm{C} 12$ & $\mathrm{C} 13$ & $1.392(2)$ \\
$\mathrm{C} 13$ & $\mathrm{C} 14$ & $1.385(3)$ \\
$\mathrm{C} 14$ & $\mathrm{C} 15$ & $1.391(2)$ \\
$\mathrm{C} 15$ & $\mathrm{C} 16$ & $1.391(2)$ \\
$\mathrm{C} 17$ & $\mathrm{C} 18$ & $1.401(2)$ \\
$\mathrm{C} 17$ & $\mathrm{C} 22$ & $1.397(2)$ \\
$\mathrm{C} 17$ & $\mathrm{P}$ & $1.8178(16)$ \\
$\mathrm{C} 18$ & $\mathrm{C} 19$ & $1.392(2)$ \\
$\mathrm{C} 19$ & $\mathrm{C} 20$ & $1.388(2)$ \\
$\mathrm{C} 20$ & $\mathrm{C} 21$ & $1.384(3)$ \\
$\mathrm{C} 21$ & $\mathrm{C} 22$ & $1.386(2)$ \\
$\mathrm{O}$ & $\mathrm{P}$ & $1.4946(11)$
\end{tabular}

Table S28: Bond Angles in ${ }^{\circ}$ for Compound 41.

\begin{tabular}{lllc}
\hline Atom & Atom & Atom & Angle $^{\circ}$ \\
\hline C2 & C1 & P & $107.24(10)$ \\
C6 & C1 & C2 & $108.63(13)$ \\
C6 & C1 & C10 & $109.18(12)$ \\
C6 & C1 & P & $114.81(10)$ \\
C10 & C1 & C2 & $108.66(12)$ \\
C10 & C1 & P & $108.16(10)$ \\
C3 & C2 & C1 & $109.91(12)$ \\
C4 & C3 & C2 & $109.54(13)$ \\
C4 & C3 & C7 & $109.35(13)$ \\
C7 & C3 & C2 & $109.35(14)$ \\
C5 & C4 & C3 & $109.68(14)$ \\
C4 & C5 & C6 & $109.84(13)$ \\
C4 & C5 & C9 & $109.24(13)$ \\
C9 & C5 & C6 & $109.63(13)$ \\
C1 & C6 & C5 & $109.98(12)$ \\
C3 & C7 & C8 & $109.36(13)$ \\
C9 & C8 & C7 & $109.61(14)$ \\
C10 & C8 & C7 & $109.21(13)$ \\
C10 & C8 & C9 & $110.00(13)$ \\
C5 & C9 & C8 & $109.42(13)$ \\
C8 & C10 & C1 & $109.85(13)$ \\
C12 & C11 & P & $116.02(12)$ \\
C16 & C11 & C12 & $118.95(15)$ \\
C16 & C11 & P & $125.02(12)$ \\
C13 & C12 & C11 & $120.97(16)$ \\
C14 & C13 & C12 & $119.46(16)$ \\
C13 & C14 & C15 & $120.23(16)$ \\
C16 & C15 & C14 & $120.35(16)$ \\
C15 & C16 & C11 & $120.02(15)$ \\
& & &
\end{tabular}

\begin{tabular}{lllc}
\hline Atom & Atom & Atom & \multicolumn{1}{c}{ Angle $^{\circ}$} \\
\hline C18 & C17 & P & $126.16(12)$ \\
C22 & C17 & C18 & $118.69(15)$ \\
C22 & C17 & P & $115.12(12)$ \\
C19 & C18 & C17 & $120.16(15)$ \\
C20 & C19 & C18 & $120.33(16)$ \\
C21 & C20 & C19 & $119.90(16)$ \\
C20 & C21 & C22 & $120.05(16)$ \\
C21 & C22 & C17 & $120.87(16)$ \\
C11 & P & C1 & $108.07(7)$ \\
C11 & P & C17 & $107.64(7)$ \\
C17 & P & C1 & $109.70(7)$ \\
O & P & C1 & $110.77(7)$ \\
O & P & C11 & $110.56(7)$ \\
O & P & C17 & $110.03(7)$
\end{tabular}


Table S29: Hydrogen Fractional Atomic Coordinates $\left(\times 10^{4}\right)$ and Equivalent Isotropic Displacement Parameters $\left(\AA^{2} \times 10^{3}\right)$ for Compound 41. $U_{e q}$ is defined as $1 / 3$ of the trace of the orthogonalised $U_{i j}$.

\begin{tabular}{|c|c|c|c|c|}
\hline Atom & & & & $U_{e q}$ \\
\hline$\overline{\mathrm{H} 2 \mathrm{~A}}$ & 4576 & 6303 & 9470 & 26 \\
\hline $\mathrm{H} 2 \mathrm{~B}$ & 4731 & 5054 & 9177 & 26 \\
\hline H3 & 7126 & 5736 & 9588 & 30 \\
\hline $\mathrm{H} 4 \mathrm{~A}$ & 6734 & 7635 & 9264 & 31 \\
\hline H4B & 8232 & 7221 & 8838 & 31 \\
\hline H5 & 6778 & 8237 & 7872 & 27 \\
\hline H6A & 4363 & 7834 & 8416 & 25 \\
\hline H6B & 4389 & 7555 & 7459 & 25 \\
\hline H7A & 7117 & 4402 & 8503 & 32 \\
\hline H7B & 8475 & 5234 & 8380 & 32 \\
\hline H8 & 7192 & 5006 & 7103 & 28 \\
\hline H9A & 8268 & 6780 & 7331 & 28 \\
\hline H9B & 6791 & 6908 & 6793 & 28 \\
\hline $\mathrm{H} 10 \mathrm{~A}$ & 4789 & 4595 & 7652 & 25 \\
\hline $\mathrm{H} 10 \mathrm{~B}$ & 4648 & 5552 & 6985 & 25 \\
\hline H12 & 1044 & 5541 & 9762 & 27 \\
\hline H13 & -147 & 6597 & 10769 & 33 \\
\hline H14 & -350 & 8504 & 10600 & 36 \\
\hline H15 & 637 & 9353 & 9433 & 35 \\
\hline H16 & 1776 & 8298 & 8410 & 28 \\
\hline H18 & 2099 & 7804 & 7052 & 25 \\
\hline H19 & 874 & 8040 & 5794 & 30 \\
\hline $\mathrm{H} 20$ & -488 & 6590 & 5217 & 35 \\
\hline H21 & -555 & 4880 & 5873 & 34 \\
\hline H22 & 670 & 4631 & 7123 & 28 \\
\hline
\end{tabular}


(42) (3-Hydroxyadamant-1-yl)diphenylphosphine oxide

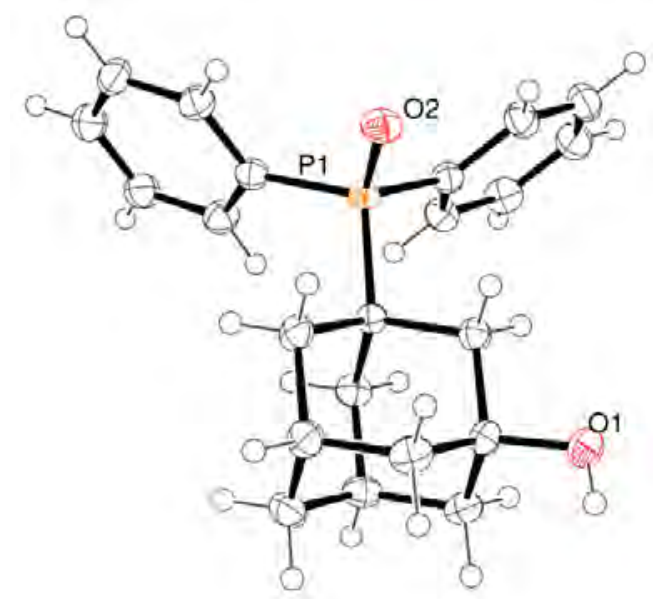

Ellipsoids are drawn at $50 \%$ probability.

Table S30. Crystal data and structure refinement for Compound 42.

CCDC No.

Empirical formula

Formula weight

Temperature

Wavelength

Crystal system

Space group

Unit cell dimensions

Volume

Z

Density (calculated)

Absorption coefficient

$\mathrm{F}(000)$

Crystal size

Theta range for data collection

Index ranges

Reflections collected

Independent reflections

Completeness to theta $=25.242^{\circ}$

Absorption correction

Max. and min. transmission

Refinement method

Data / restraints / parameters
1053892

$\mathrm{C} 22 \mathrm{H} 25 \mathrm{O} 2 \mathrm{P}$

352.39

150(2) K

$0.71073 \AA$

Monoclinic

$\mathrm{P} 2 / 1 / \mathrm{n}$

$\mathrm{a}=8.6126(17) \AA$

$\alpha=90^{\circ}$.

$\mathrm{b}=12.987(3) \AA$

$\beta=99.46(3)^{\circ}$.

$\mathrm{c}=16.000(3) \AA$

$\gamma=90^{\circ}$.

1765.4(6) $\AA^{3}$

4

$1.326 \mathrm{Mg} / \mathrm{m}^{3}$

$0.168 \mathrm{~mm}^{-1}$

752

$0.500 \times 0.250 \times 0.200 \mathrm{~mm}^{3}$

2.031 to $30.070^{\circ}$.

$-12<=\mathrm{h}<=12,-18<=\mathrm{k}<=18,-21<=\mathrm{l}<=22$

47166

$5146[\mathrm{R}(\mathrm{int})=0.0654]$

$99.9 \%$

Empirical

0.7460 and 0.5991

Full-matrix least-squares on $\mathrm{F}^{2}$

$5146 / 0 / 228$ 
Goodness-of-fit on $\mathrm{F}^{2}$

Final $\mathrm{R}$ indices [I $>2 \operatorname{sigma}(\mathrm{I})]$

$\mathrm{R}$ indices (all data)

Extinction coefficient

Largest diff. peak and hole
1.094

$\mathrm{R} 1=0.0397, \mathrm{wR} 2=0.1078$

$\mathrm{R} 1=0.0547, \mathrm{wR} 2=0.1133$

$\mathrm{n} / \mathrm{a}$

0.338 and -0.321 e. $\AA^{-3}$ 
Table S31. Bond lengths $[\AA]$ and angles $\left[{ }^{\circ}\right]$ for Compound 42.

\begin{tabular}{|c|c|}
\hline $\mathrm{P}(1)-\mathrm{O}(2)$ & $1.4936(10)$ \\
\hline $\mathrm{P}(1)-\mathrm{C}(11)$ & $1.8050(13)$ \\
\hline $\mathrm{P}(1)-\mathrm{C}(17)$ & $1.8107(14)$ \\
\hline $\mathrm{P}(1)-\mathrm{C}(1)$ & $1.8317(13)$ \\
\hline $\mathrm{O}(1)-\mathrm{C}(5)$ & $1.4268(15)$ \\
\hline $\mathrm{O}(1)-\mathrm{H}(1)$ & $0.81(2)$ \\
\hline$C(1)-C(2)$ & $1.5414(17)$ \\
\hline$C(1)-C(10)$ & $1.5459(16)$ \\
\hline$C(1)-C(6)$ & $1.5483(17)$ \\
\hline$C(2)-C(3)$ & $1.5421(18)$ \\
\hline $\mathrm{C}(2)-\mathrm{H}(2 \mathrm{~A})$ & 0.9900 \\
\hline $\mathrm{C}(2)-\mathrm{H}(2 \mathrm{~B})$ & 0.9900 \\
\hline$C(3)-C(9)$ & $1.5317(19)$ \\
\hline$C(3)-C(4)$ & $1.5325(19)$ \\
\hline $\mathrm{C}(3)-\mathrm{H}(3)$ & 1.0000 \\
\hline$C(4)-C(5)$ & $1.5278(19)$ \\
\hline $\mathrm{C}(4)-\mathrm{H}(4 \mathrm{~A})$ & 0.9900 \\
\hline $\mathrm{C}(4)-\mathrm{H}(4 \mathrm{~B})$ & 0.9900 \\
\hline$C(5)-C(6)$ & $1.5255(18)$ \\
\hline$C(5)-C(7)$ & $1.5272(18)$ \\
\hline $\mathrm{C}(6)-\mathrm{H}(6 \mathrm{~A})$ & 0.9900 \\
\hline $\mathrm{C}(6)-\mathrm{H}(6 \mathrm{~B})$ & 0.9900 \\
\hline $\mathrm{C}(8)-\mathrm{C}(9)$ & $1.527(2)$ \\
\hline $\mathrm{C}(8)-\mathrm{C}(7)$ & $1.5312(19)$ \\
\hline$C(8)-C(10)$ & $1.5395(19)$ \\
\hline $\mathrm{C}(8)-\mathrm{H}(8)$ & 1.0000 \\
\hline $\mathrm{C}(7)-\mathrm{H}(7 \mathrm{~A})$ & 0.9900 \\
\hline $\mathrm{C}(7)-\mathrm{H}(7 \mathrm{~B})$ & 0.9900 \\
\hline $\mathrm{C}(9)-\mathrm{H}(9 \mathrm{~A})$ & 0.9900 \\
\hline C(9)-H(9B) & 0.9900 \\
\hline $\mathrm{C}(10)-\mathrm{H}(10 \mathrm{~A})$ & 0.9900 \\
\hline $\mathrm{C}(10)-\mathrm{H}(10 \mathrm{~B})$ & 0.9900 \\
\hline$C(11)-C(12)$ & $1.3958(19)$ \\
\hline$C(11)-C(16)$ & $1.3985(18)$ \\
\hline$C(12)-C(13)$ & $1.3883(18)$ \\
\hline $\mathrm{C}(12)-\mathrm{H}(12)$ & 0.9500 \\
\hline$C(13)-C(14)$ & $1.388(2)$ \\
\hline C(13)-H(13) & 0.9500 \\
\hline$C(14)-C(15)$ & $1.373(2)$ \\
\hline
\end{tabular}




\begin{tabular}{|c|c|}
\hline $\mathrm{C}(14)-\mathrm{H}(14)$ & 0.9500 \\
\hline$C(15)-C(16)$ & $1.3904(19)$ \\
\hline $\mathrm{C}(15)-\mathrm{H}(15)$ & 0.9500 \\
\hline $\mathrm{C}(16)-\mathrm{H}(16)$ & 0.9500 \\
\hline $\mathrm{C}(17)-\mathrm{C}(18)$ & $1.3942(18)$ \\
\hline $\mathrm{C}(17)-\mathrm{C}(22)$ & $1.3975(18)$ \\
\hline $\mathrm{C}(18)-\mathrm{C}(19)$ & $1.3984(19)$ \\
\hline $\mathrm{C}(18)-\mathrm{H}(18)$ & 0.9500 \\
\hline$C(19)-C(20)$ & $1.384(2)$ \\
\hline C(19)-H(19) & 0.9500 \\
\hline$C(20)-C(21)$ & $1.390(2)$ \\
\hline $\mathrm{C}(20)-\mathrm{H}(20)$ & 0.9500 \\
\hline $\mathrm{C}(21)-\mathrm{C}(22)$ & $1.3958(19)$ \\
\hline $\mathrm{C}(21)-\mathrm{H}(21)$ & 0.9500 \\
\hline $\mathrm{C}(22)-\mathrm{H}(22)$ & 0.9500 \\
\hline $\mathrm{O}(2)-\mathrm{P}(1)-\mathrm{C}(11)$ & $109.70(6)$ \\
\hline $\mathrm{O}(2)-\mathrm{P}(1)-\mathrm{C}(17)$ & $110.04(6)$ \\
\hline $\mathrm{C}(11)-\mathrm{P}(1)-\mathrm{C}(17)$ & $106.76(6)$ \\
\hline $\mathrm{O}(2)-\mathrm{P}(1)-\mathrm{C}(1)$ & $110.48(6)$ \\
\hline $\mathrm{C}(11)-\mathrm{P}(1)-\mathrm{C}(1)$ & $109.23(6)$ \\
\hline $\mathrm{C}(17)-\mathrm{P}(1)-\mathrm{C}(1)$ & $110.55(6)$ \\
\hline $\mathrm{C}(5)-\mathrm{O}(1)-\mathrm{H}(1)$ & 109.5 \\
\hline $\mathrm{C}(2)-\mathrm{C}(1)-\mathrm{C}(10)$ & $109.09(10)$ \\
\hline$C(2)-C(1)-C(6)$ & $108.79(10)$ \\
\hline$C(10)-C(1)-C(6)$ & $108.89(10)$ \\
\hline $\mathrm{C}(2)-\mathrm{C}(1)-\mathrm{P}(1)$ & $115.54(8)$ \\
\hline $\mathrm{C}(10)-\mathrm{C}(1)-\mathrm{P}(1)$ & $107.01(8)$ \\
\hline$C(6)-C(1)-P(1)$ & $107.36(8)$ \\
\hline$C(1)-C(2)-C(3)$ & $109.50(10)$ \\
\hline $\mathrm{C}(1)-\mathrm{C}(2)-\mathrm{H}(2 \mathrm{~A})$ & 109.8 \\
\hline $\mathrm{C}(3)-\mathrm{C}(2)-\mathrm{H}(2 \mathrm{~A})$ & 109.8 \\
\hline $\mathrm{C}(1)-\mathrm{C}(2)-\mathrm{H}(2 \mathrm{~B})$ & 109.8 \\
\hline $\mathrm{C}(3)-\mathrm{C}(2)-\mathrm{H}(2 \mathrm{~B})$ & 109.8 \\
\hline $\mathrm{H}(2 \mathrm{~A})-\mathrm{C}(2)-\mathrm{H}(2 \mathrm{~B})$ & 108.2 \\
\hline$C(9)-C(3)-C(4)$ & $109.64(11)$ \\
\hline $\mathrm{C}(9)-\mathrm{C}(3)-\mathrm{C}(2)$ & $109.47(11)$ \\
\hline$C(4)-C(3)-C(2)$ & $109.54(10)$ \\
\hline $\mathrm{C}(9)-\mathrm{C}(3)-\mathrm{H}(3)$ & 109.4 \\
\hline $\mathrm{C}(4)-\mathrm{C}(3)-\mathrm{H}(3)$ & 109.4 \\
\hline $\mathrm{C}(2)-\mathrm{C}(3)-\mathrm{H}(3)$ & 109.4 \\
\hline
\end{tabular}




\begin{tabular}{|c|c|}
\hline$C(5)-C(4)-C(3)$ & $109.72(10)$ \\
\hline $\mathrm{C}(5)-\mathrm{C}(4)-\mathrm{H}(4 \mathrm{~A})$ & 109.7 \\
\hline $\mathrm{C}(3)-\mathrm{C}(4)-\mathrm{H}(4 \mathrm{~A})$ & 109.7 \\
\hline $\mathrm{C}(5)-\mathrm{C}(4)-\mathrm{H}(4 \mathrm{~B})$ & 109.7 \\
\hline $\mathrm{C}(3)-\mathrm{C}(4)-\mathrm{H}(4 \mathrm{~B})$ & 109.7 \\
\hline $\mathrm{H}(4 \mathrm{~A})-\mathrm{C}(4)-\mathrm{H}(4 \mathrm{~B})$ & 108.2 \\
\hline $\mathrm{O}(1)-\mathrm{C}(5)-\mathrm{C}(6)$ & $106.34(10)$ \\
\hline $\mathrm{O}(1)-\mathrm{C}(5)-\mathrm{C}(7)$ & $111.01(11)$ \\
\hline$C(6)-C(5)-C(7)$ & $109.33(11)$ \\
\hline $\mathrm{O}(1)-\mathrm{C}(5)-\mathrm{C}(4)$ & $111.24(11)$ \\
\hline$C(6)-C(5)-C(4)$ & $109.34(10)$ \\
\hline$C(7)-C(5)-C(4)$ & $109.52(10)$ \\
\hline$C(5)-C(6)-C(1)$ & $110.20(10)$ \\
\hline $\mathrm{C}(5)-\mathrm{C}(6)-\mathrm{H}(6 \mathrm{~A})$ & 109.6 \\
\hline $\mathrm{C}(1)-\mathrm{C}(6)-\mathrm{H}(6 \mathrm{~A})$ & 109.6 \\
\hline $\mathrm{C}(5)-\mathrm{C}(6)-\mathrm{H}(6 \mathrm{~B})$ & 109.6 \\
\hline $\mathrm{C}(1)-\mathrm{C}(6)-\mathrm{H}(6 \mathrm{~B})$ & 109.6 \\
\hline $\mathrm{H}(6 \mathrm{~A})-\mathrm{C}(6)-\mathrm{H}(6 \mathrm{~B})$ & 108.1 \\
\hline$C(9)-C(8)-C(7)$ & $109.87(11)$ \\
\hline $\mathrm{C}(9)-\mathrm{C}(8)-\mathrm{C}(10)$ & $109.82(11)$ \\
\hline $\mathrm{C}(7)-\mathrm{C}(8)-\mathrm{C}(10)$ & $109.06(11)$ \\
\hline $\mathrm{C}(9)-\mathrm{C}(8)-\mathrm{H}(8)$ & 109.4 \\
\hline $\mathrm{C}(7)-\mathrm{C}(8)-\mathrm{H}(8)$ & 109.4 \\
\hline $\mathrm{C}(10)-\mathrm{C}(8)-\mathrm{H}(8)$ & 109.4 \\
\hline$C(5)-C(7)-C(8)$ & $109.91(10)$ \\
\hline $\mathrm{C}(5)-\mathrm{C}(7)-\mathrm{H}(7 \mathrm{~A})$ & 109.7 \\
\hline $\mathrm{C}(8)-\mathrm{C}(7)-\mathrm{H}(7 \mathrm{~A})$ & 109.7 \\
\hline $\mathrm{C}(5)-\mathrm{C}(7)-\mathrm{H}(7 \mathrm{~B})$ & 109.7 \\
\hline $\mathrm{C}(8)-\mathrm{C}(7)-\mathrm{H}(7 \mathrm{~B})$ & 109.7 \\
\hline $\mathrm{H}(7 \mathrm{~A})-\mathrm{C}(7)-\mathrm{H}(7 \mathrm{~B})$ & 108.2 \\
\hline $\mathrm{C}(8)-\mathrm{C}(9)-\mathrm{C}(3)$ & $109.35(10)$ \\
\hline $\mathrm{C}(8)-\mathrm{C}(9)-\mathrm{H}(9 \mathrm{~A})$ & 109.8 \\
\hline $\mathrm{C}(3)-\mathrm{C}(9)-\mathrm{H}(9 \mathrm{~A})$ & 109.8 \\
\hline $\mathrm{C}(8)-\mathrm{C}(9)-\mathrm{H}(9 \mathrm{~B})$ & 109.8 \\
\hline $\mathrm{C}(3)-\mathrm{C}(9)-\mathrm{H}(9 \mathrm{~B})$ & 109.8 \\
\hline $\mathrm{H}(9 \mathrm{~A})-\mathrm{C}(9)-\mathrm{H}(9 \mathrm{~B})$ & 108.3 \\
\hline $\mathrm{C}(8)-\mathrm{C}(10)-\mathrm{C}(1)$ & $109.44(10)$ \\
\hline $\mathrm{C}(8)-\mathrm{C}(10)-\mathrm{H}(10 \mathrm{~A})$ & 109.8 \\
\hline$C(1)-C(10)-H(10 A)$ & 109.8 \\
\hline $\mathrm{C}(8)-\mathrm{C}(10)-\mathrm{H}(10 \mathrm{~B})$ & 109.8 \\
\hline $\mathrm{C}(1)-\mathrm{C}(10)-\mathrm{H}(10 \mathrm{~B})$ & 109.8 \\
\hline
\end{tabular}




\begin{tabular}{|c|c|}
\hline $\mathrm{H}(10 \mathrm{~A})-\mathrm{C}(10)-\mathrm{H}(10 \mathrm{~B})$ & 108.2 \\
\hline $\mathrm{C}(12)-\mathrm{C}(11)-\mathrm{C}(16)$ & $118.66(12)$ \\
\hline $\mathrm{C}(12)-\mathrm{C}(11)-\mathrm{P}(1)$ & $125.45(10)$ \\
\hline $\mathrm{C}(16)-\mathrm{C}(11)-\mathrm{P}(1)$ & $115.87(10)$ \\
\hline$C(13)-C(12)-C(11)$ & $120.87(12)$ \\
\hline $\mathrm{C}(13)-\mathrm{C}(12)-\mathrm{H}(12)$ & 119.6 \\
\hline $\mathrm{C}(11)-\mathrm{C}(12)-\mathrm{H}(12)$ & 119.6 \\
\hline $\mathrm{C}(12)-\mathrm{C}(13)-\mathrm{C}(14)$ & $119.43(13)$ \\
\hline $\mathrm{C}(12)-\mathrm{C}(13)-\mathrm{H}(13)$ & 120.3 \\
\hline $\mathrm{C}(14)-\mathrm{C}(13)-\mathrm{H}(13)$ & 120.3 \\
\hline$C(15)-C(14)-C(13)$ & $120.53(13)$ \\
\hline $\mathrm{C}(15)-\mathrm{C}(14)-\mathrm{H}(14)$ & 119.7 \\
\hline $\mathrm{C}(13)-\mathrm{C}(14)-\mathrm{H}(14)$ & 119.7 \\
\hline$C(14)-C(15)-C(16)$ & $120.27(13)$ \\
\hline $\mathrm{C}(14)-\mathrm{C}(15)-\mathrm{H}(15)$ & 119.9 \\
\hline $\mathrm{C}(16)-\mathrm{C}(15)-\mathrm{H}(15)$ & 119.9 \\
\hline$C(15)-C(16)-C(11)$ & $120.24(13)$ \\
\hline $\mathrm{C}(15)-\mathrm{C}(16)-\mathrm{H}(16)$ & 119.9 \\
\hline $\mathrm{C}(11)-\mathrm{C}(16)-\mathrm{H}(16)$ & 119.9 \\
\hline $\mathrm{C}(18)-\mathrm{C}(17)-\mathrm{C}(22)$ & $118.29(12)$ \\
\hline $\mathrm{C}(18)-\mathrm{C}(17)-\mathrm{P}(1)$ & $116.22(10)$ \\
\hline $\mathrm{C}(22)-\mathrm{C}(17)-\mathrm{P}(1)$ & $125.38(10)$ \\
\hline$C(17)-C(18)-C(19)$ & $120.93(13)$ \\
\hline $\mathrm{C}(17)-\mathrm{C}(18)-\mathrm{H}(18)$ & 119.5 \\
\hline C(19)-C(18)-H(18) & 119.5 \\
\hline $\mathrm{C}(20)-\mathrm{C}(19)-\mathrm{C}(18)$ & $119.59(13)$ \\
\hline $\mathrm{C}(20)-\mathrm{C}(19)-\mathrm{H}(19)$ & 120.2 \\
\hline $\mathrm{C}(18)-\mathrm{C}(19)-\mathrm{H}(19)$ & 120.2 \\
\hline$C(19)-C(20)-C(21)$ & $120.75(13)$ \\
\hline $\mathrm{C}(19)-\mathrm{C}(20)-\mathrm{H}(20)$ & 119.6 \\
\hline $\mathrm{C}(21)-\mathrm{C}(20)-\mathrm{H}(20)$ & 119.6 \\
\hline $\mathrm{C}(20)-\mathrm{C}(21)-\mathrm{C}(22)$ & $119.04(13)$ \\
\hline $\mathrm{C}(20)-\mathrm{C}(21)-\mathrm{H}(21)$ & 120.5 \\
\hline $\mathrm{C}(22)-\mathrm{C}(21)-\mathrm{H}(21)$ & 120.5 \\
\hline $\mathrm{C}(21)-\mathrm{C}(22)-\mathrm{C}(17)$ & $121.39(13)$ \\
\hline $\mathrm{C}(21)-\mathrm{C}(22)-\mathrm{H}(22)$ & 119.3 \\
\hline $\mathrm{C}(17)-\mathrm{C}(22)-\mathrm{H}(22)$ & 119.3 \\
\hline
\end{tabular}




\section{(43) 4-Diamantyldiphenylphosphine oxide}

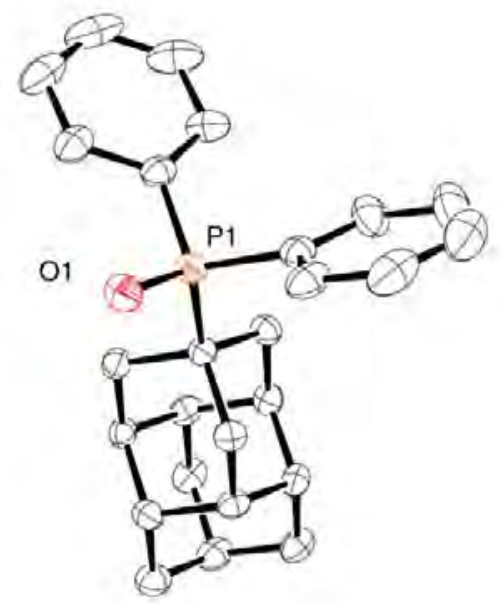

Ellipsoids are drawn at $50 \%$ probability.

Table S32. Crystal data and structure refinement for Compound 43.

CCDC No.

Empirical formula

Formula weight

Temperature

Wavelength

Crystal system

Space group

Unit cell dimensions

Volume

Z

Density (calculated)

Absorption coefficient

$\mathrm{F}(000)$

Crystal size

Theta range for data collection

Index ranges

Reflections collected

Independent reflections

Completeness to theta $=25.020^{\circ}$

Absorption correction

Max. and min. transmission

Refinement method

Data / restraints / parameters
1053890

C27 H33 O2 P

420.50

150(2) K

$0.71073 \AA$

Orthorhombic

Pnma

$\mathrm{a}=11.550(2) \AA$

$\alpha=90^{\circ}$.

$\mathrm{b}=12.931(3) \AA$

$\beta=90^{\circ}$.

$\mathrm{c}=14.982(3) \AA$

$\gamma=90^{\circ}$.

2237.6(8) $\AA^{3}$

4

$1.248 \mathrm{Mg} / \mathrm{m}^{3}$

$0.144 \mathrm{~mm}^{-1}$

904

$0.700 \times 0.300 \times 0.250 \mathrm{~mm}^{3}$

2.226 to $25.020^{\circ}$.

$-13<=\mathrm{h}<=13,-15<=\mathrm{k}<=15,-17<=1<=17$

22179

$2074[\mathrm{R}(\mathrm{int})=0.0674]$

$100.0 \%$

Empirical

0.7456 and 0.5841

Full-matrix least-squares on $\mathrm{F}^{2}$

2074 / 0 / 155 
Goodness-of-fit on $\mathrm{F}^{2}$

Final $\mathrm{R}$ indices [I $>2 \operatorname{sigma}(\mathrm{I})]$

$\mathrm{R}$ indices (all data)

Extinction coefficient

Largest diff. peak and hole
1.042

$\mathrm{R} 1=0.0382, \mathrm{wR} 2=0.0985$

$\mathrm{R} 1=0.0514, \mathrm{wR} 2=0.1072$

$\mathrm{n} / \mathrm{a}$

0.322 and -0.338 e. $\AA^{-3}$ 
Table S33. Bond lengths $[\AA]$ and angles $\left[{ }^{\circ}\right]$ for Compound 43

\begin{tabular}{|c|c|}
\hline $\mathrm{P}(1)-\mathrm{O}(1)$ & $1.5001(17)$ \\
\hline $\mathrm{P}(1)-\mathrm{C}(11) \# 1$ & $1.8076(18)$ \\
\hline $\mathrm{P}(1)-\mathrm{C}(11)$ & $1.8076(18)$ \\
\hline $\mathrm{P}(1)-\mathrm{C}(1)$ & $1.833(2)$ \\
\hline$C(1)-C(2)$ & $1.543(3)$ \\
\hline $\mathrm{C}(1)-\mathrm{C}(5) \# 1$ & $1.546(2)$ \\
\hline$C(1)-C(5)$ & $1.546(2)$ \\
\hline$C(2)-C(3)$ & $1.539(3)$ \\
\hline $\mathrm{C}(2)-\mathrm{H}(2 \mathrm{~A})$ & 0.9900 \\
\hline $\mathrm{C}(2)-\mathrm{H}(2 \mathrm{~B})$ & 0.9900 \\
\hline $\mathrm{O}(2)-\mathrm{C}(17)$ & $1.367(4)$ \\
\hline $\mathrm{O}(2)-\mathrm{H}(1)$ & $0.97(6)$ \\
\hline$C(3)-C(4) \# 1$ & $1.534(2)$ \\
\hline$C(3)-C(4)$ & $1.534(2)$ \\
\hline $\mathrm{C}(3)-\mathrm{H}(3)$ & 1.0000 \\
\hline$C(5)-C(6)$ & $1.532(2)$ \\
\hline $\mathrm{C}(5)-\mathrm{H}(5 \mathrm{~A})$ & 0.9900 \\
\hline $\mathrm{C}(5)-\mathrm{H}(5 \mathrm{~B})$ & 0.9900 \\
\hline$C(4)-C(10)$ & $1.534(2)$ \\
\hline $\mathrm{C}(4)-\mathrm{C}(6) \# 1$ & $1.542(2)$ \\
\hline $\mathrm{C}(4)-\mathrm{H}(4)$ & 1.0000 \\
\hline$C(6)-C(7)$ & $1.541(2)$ \\
\hline$C(6)-C(4) \# 1$ & $1.542(2)$ \\
\hline $\mathrm{C}(6)-\mathrm{H}(6)$ & 1.0000 \\
\hline$C(7)-C(8)$ & $1.535(3)$ \\
\hline$C(7)-C(6) \# 1$ & $1.541(2)$ \\
\hline $\mathrm{C}(7)-\mathrm{H}(7)$ & 1.0000 \\
\hline $\mathrm{C}(9)-\mathrm{C}(8)$ & $1.525(4)$ \\
\hline $\mathrm{C}(9)-\mathrm{C}(10) \# 1$ & $1.533(2)$ \\
\hline $\mathrm{C}(9)-\mathrm{C}(10)$ & $1.533(2)$ \\
\hline $\mathrm{C}(9)-\mathrm{H}(9)$ & 1.0000 \\
\hline $\mathrm{C}(8)-\mathrm{H}(8 \mathrm{~A})$ & 0.9900 \\
\hline $\mathrm{C}(8)-\mathrm{H}(8 \mathrm{~B})$ & 0.9900 \\
\hline $\mathrm{C}(10)-\mathrm{H}(10 \mathrm{~A})$ & 0.9900 \\
\hline $\mathrm{C}(10)-\mathrm{H}(10 \mathrm{~B})$ & 0.9900 \\
\hline$C(11)-C(12)$ & $1.397(2)$ \\
\hline $\mathrm{C}(11)-\mathrm{C}(16)$ & $1.400(3)$ \\
\hline $\mathrm{C}(12)-\mathrm{C}(13)$ & $1.388(3)$ \\
\hline
\end{tabular}




\begin{tabular}{|c|c|}
\hline $\mathrm{C}(12)-\mathrm{H}(12)$ & 0.9500 \\
\hline C(13)-C(14) & $1.373(3)$ \\
\hline $\mathrm{C}(13)-\mathrm{H}(13)$ & 0.9500 \\
\hline$C(14)-C(15)$ & $1.380(3)$ \\
\hline $\mathrm{C}(14)-\mathrm{H}(14)$ & 0.9500 \\
\hline$C(15)-C(16)$ & $1.391(3)$ \\
\hline $\mathrm{C}(15)-\mathrm{H}(15)$ & 0.9500 \\
\hline $\mathrm{C}(16)-\mathrm{H}(16)$ & 0.9500 \\
\hline $\mathrm{C}(17)-\mathrm{H}(17 \mathrm{~A})$ & 0.9800 \\
\hline C(17)-H(17B) & 0.9800 \\
\hline C(17)-H(17C) & 0.9800 \\
\hline $\mathrm{O}(1)-\mathrm{P}(1)-\mathrm{C}(11) \# 1$ & 109.30(7) \\
\hline $\mathrm{O}(1)-\mathrm{P}(1)-\mathrm{C}(11)$ & $109.30(7)$ \\
\hline $\mathrm{C}(11) \# 1-\mathrm{P}(1)-\mathrm{C}(11)$ & $110.03(12)$ \\
\hline $\mathrm{O}(1)-\mathrm{P}(1)-\mathrm{C}(1)$ & $111.41(10)$ \\
\hline $\mathrm{C}(11) \# 1-\mathrm{P}(1)-\mathrm{C}(1)$ & $108.39(7)$ \\
\hline $\mathrm{C}(11)-\mathrm{P}(1)-\mathrm{C}(1)$ & $108.39(7)$ \\
\hline$C(2)-C(1)-C(5) \# 1$ & $108.70(12)$ \\
\hline $\mathrm{C}(2)-\mathrm{C}(1)-\mathrm{C}(5)$ & $108.70(12)$ \\
\hline C(5)\#1-C(1)-C(5) & $109.13(18)$ \\
\hline $\mathrm{C}(2)-\mathrm{C}(1)-\mathrm{P}(1)$ & $114.14(16)$ \\
\hline $\mathrm{C}(5) \# 1-\mathrm{C}(1)-\mathrm{P}(1)$ & $108.04(10)$ \\
\hline $\mathrm{C}(5)-\mathrm{C}(1)-\mathrm{P}(1)$ & $108.05(10)$ \\
\hline$C(3)-C(2)-C(1)$ & 109.63(19) \\
\hline $\mathrm{C}(3)-\mathrm{C}(2)-\mathrm{H}(2 \mathrm{~A})$ & 109.7 \\
\hline $\mathrm{C}(1)-\mathrm{C}(2)-\mathrm{H}(2 \mathrm{~A})$ & 109.7 \\
\hline $\mathrm{C}(3)-\mathrm{C}(2)-\mathrm{H}(2 \mathrm{~B})$ & 109.7 \\
\hline $\mathrm{C}(1)-\mathrm{C}(2)-\mathrm{H}(2 \mathrm{~B})$ & 109.7 \\
\hline $\mathrm{H}(2 \mathrm{~A})-\mathrm{C}(2)-\mathrm{H}(2 \mathrm{~B})$ & 108.2 \\
\hline $\mathrm{C}(17)-\mathrm{O}(2)-\mathrm{H}(1)$ & $116(3)$ \\
\hline $\mathrm{C}(4) \# 1-\mathrm{C}(3)-\mathrm{C}(4)$ & 109.13(19) \\
\hline $\mathrm{C}(4) \# 1-\mathrm{C}(3)-\mathrm{C}(2)$ & $110.57(12)$ \\
\hline $\mathrm{C}(4)-\mathrm{C}(3)-\mathrm{C}(2)$ & $110.57(12)$ \\
\hline $\mathrm{C}(4) \# 1-\mathrm{C}(3)-\mathrm{H}(3)$ & 108.8 \\
\hline $\mathrm{C}(4)-\mathrm{C}(3)-\mathrm{H}(3)$ & 108.8 \\
\hline $\mathrm{C}(2)-\mathrm{C}(3)-\mathrm{H}(3)$ & 108.8 \\
\hline$C(6)-C(5)-C(1)$ & $109.76(13)$ \\
\hline $\mathrm{C}(6)-\mathrm{C}(5)-\mathrm{H}(5 \mathrm{~A})$ & 109.7 \\
\hline $\mathrm{C}(1)-\mathrm{C}(5)-\mathrm{H}(5 \mathrm{~A})$ & 109.7 \\
\hline $\mathrm{C}(6)-\mathrm{C}(5)-\mathrm{H}(5 \mathrm{~B})$ & 109.7 \\
\hline
\end{tabular}




\begin{tabular}{|c|c|}
\hline $\mathrm{C}(1)-\mathrm{C}(5)-\mathrm{H}(5 \mathrm{~B})$ & 109.7 \\
\hline $\mathrm{H}(5 \mathrm{~A})-\mathrm{C}(5)-\mathrm{H}(5 \mathrm{~B})$ & 108.2 \\
\hline $\mathrm{C}(10)-\mathrm{C}(4)-\mathrm{C}(3)$ & $110.62(14)$ \\
\hline $\mathrm{C}(10)-\mathrm{C}(4)-\mathrm{C}(6) \# 1$ & $110.05(14)$ \\
\hline$C(3)-C(4)-C(6) \# 1$ & $108.26(13)$ \\
\hline $\mathrm{C}(10)-\mathrm{C}(4)-\mathrm{H}(4)$ & 109.3 \\
\hline $\mathrm{C}(3)-\mathrm{C}(4)-\mathrm{H}(4)$ & 109.3 \\
\hline $\mathrm{C}(6) \# 1-\mathrm{C}(4)-\mathrm{H}(4)$ & 109.3 \\
\hline$C(5)-C(6)-C(7)$ & $110.60(14)$ \\
\hline$C(5)-C(6)-C(4) \# 1$ & $110.59(14)$ \\
\hline$C(7)-C(6)-C(4) \# 1$ & $108.48(14)$ \\
\hline $\mathrm{C}(5)-\mathrm{C}(6)-\mathrm{H}(6)$ & 109.0 \\
\hline $\mathrm{C}(7)-\mathrm{C}(6)-\mathrm{H}(6)$ & 109.0 \\
\hline $\mathrm{C}(4) \# 1-\mathrm{C}(6)-\mathrm{H}(6)$ & 109.0 \\
\hline$C(8)-C(7)-C(6)$ & $110.39(13)$ \\
\hline $\mathrm{C}(8)-\mathrm{C}(7)-\mathrm{C}(6) \# 1$ & $110.38(13)$ \\
\hline$C(6)-C(7)-C(6) \# 1$ & $108.56(19)$ \\
\hline $\mathrm{C}(8)-\mathrm{C}(7)-\mathrm{H}(7)$ & 109.2 \\
\hline $\mathrm{C}(6)-\mathrm{C}(7)-\mathrm{H}(7)$ & 109.2 \\
\hline $\mathrm{C}(6) \# 1-\mathrm{C}(7)-\mathrm{H}(7)$ & 109.2 \\
\hline $\mathrm{C}(8)-\mathrm{C}(9)-\mathrm{C}(10) \# 1$ & $109.20(13)$ \\
\hline C(8)-C(9)-C(10) & $109.20(13)$ \\
\hline $\mathrm{C}(10) \# 1-\mathrm{C}(9)-\mathrm{C}(10)$ & $109.5(2)$ \\
\hline $\mathrm{C}(8)-\mathrm{C}(9)-\mathrm{H}(9)$ & 109.6 \\
\hline $\mathrm{C}(10) \# 1-\mathrm{C}(9)-\mathrm{H}(9)$ & 109.6 \\
\hline $\mathrm{C}(10)-\mathrm{C}(9)-\mathrm{H}(9)$ & 109.6 \\
\hline $\mathrm{C}(9)-\mathrm{C}(8)-\mathrm{C}(7)$ & $109.7(2)$ \\
\hline $\mathrm{C}(9)-\mathrm{C}(8)-\mathrm{H}(8 \mathrm{~A})$ & 109.7 \\
\hline $\mathrm{C}(7)-\mathrm{C}(8)-\mathrm{H}(8 \mathrm{~A})$ & 109.7 \\
\hline $\mathrm{C}(9)-\mathrm{C}(8)-\mathrm{H}(8 \mathrm{~B})$ & 109.7 \\
\hline $\mathrm{C}(7)-\mathrm{C}(8)-\mathrm{H}(8 \mathrm{~B})$ & 109.7 \\
\hline $\mathrm{H}(8 \mathrm{~A})-\mathrm{C}(8)-\mathrm{H}(8 \mathrm{~B})$ & 108.2 \\
\hline$C(9)-C(10)-C(4)$ & $109.72(15)$ \\
\hline $\mathrm{C}(9)-\mathrm{C}(10)-\mathrm{H}(10 \mathrm{~A})$ & 109.7 \\
\hline $\mathrm{C}(4)-\mathrm{C}(10)-\mathrm{H}(10 \mathrm{~A})$ & 109.7 \\
\hline $\mathrm{C}(9)-\mathrm{C}(10)-\mathrm{H}(10 \mathrm{~B})$ & 109.7 \\
\hline $\mathrm{C}(4)-\mathrm{C}(10)-\mathrm{H}(10 \mathrm{~B})$ & 109.7 \\
\hline $\mathrm{H}(10 \mathrm{~A})-\mathrm{C}(10)-\mathrm{H}(10 \mathrm{~B})$ & 108.2 \\
\hline$C(12)-C(11)-C(16)$ & $118.54(17)$ \\
\hline $\mathrm{C}(12)-\mathrm{C}(11)-\mathrm{P}(1)$ & $115.90(14)$ \\
\hline $\mathrm{C}(16)-\mathrm{C}(11)-\mathrm{P}(1)$ & $125.54(14)$ \\
\hline
\end{tabular}




$\begin{array}{ll}\mathrm{C}(13)-\mathrm{C}(12)-\mathrm{C}(11) & 120.69(19) \\ \mathrm{C}(13)-\mathrm{C}(12)-\mathrm{H}(12) & 119.7 \\ \mathrm{C}(11)-\mathrm{C}(12)-\mathrm{H}(12) & 119.7 \\ \mathrm{C}(14)-\mathrm{C}(13)-\mathrm{C}(12) & 120.2(2) \\ \mathrm{C}(14)-\mathrm{C}(13)-\mathrm{H}(13) & 119.9 \\ \mathrm{C}(12)-\mathrm{C}(13)-\mathrm{H}(13) & 119.9 \\ \mathrm{C}(13)-\mathrm{C}(14)-\mathrm{C}(15) & 120.0(2) \\ \mathrm{C}(13)-\mathrm{C}(14)-\mathrm{H}(14) & 120.0 \\ \mathrm{C}(15)-\mathrm{C}(14)-\mathrm{H}(14) & 120.0 \\ \mathrm{C}(14)-\mathrm{C}(15)-\mathrm{C}(16) & 120.7(2) \\ \mathrm{C}(14)-\mathrm{C}(15)-\mathrm{H}(15) & 119.7 \\ \mathrm{C}(16)-\mathrm{C}(15)-\mathrm{H}(15) & 119.7 \\ \mathrm{C}(15)-\mathrm{C}(16)-\mathrm{C}(11) & 119.9(2) \\ \mathrm{C}(15)-\mathrm{C}(16)-\mathrm{H}(16) & 120.1 \\ \mathrm{C}(11)-\mathrm{C}(16)-\mathrm{H}(16) & 120.1 \\ \mathrm{O}(2)-\mathrm{C}(17)-\mathrm{H}(17 \mathrm{~A}) & 109.5 \\ \mathrm{O}(2)-\mathrm{C}(17)-\mathrm{H}(17 \mathrm{~B}) & 109.5 \\ \mathrm{H}(17 \mathrm{~A})-\mathrm{C}(17)-\mathrm{H}(17 \mathrm{~B}) & 109.5 \\ \mathrm{O}(2)-\mathrm{C}(17)-\mathrm{H}(17 \mathrm{C}) & 109.5 \\ \mathrm{H}(17 \mathrm{~A})-\mathrm{C}(17)-\mathrm{H}(17 \mathrm{C}) & 109.5 \\ \mathrm{H}(17 \mathrm{~B})-\mathrm{C}(17)-\mathrm{H}(17 \mathrm{C}) & 109.5 \\ & \\ & \end{array}$

Symmetry transformations used to generate equivalent atoms:

\#1 x,-y+3/2,z 


\section{(44) (9-hydroxydiamant-4-yl)diphenylphosphineoxide}

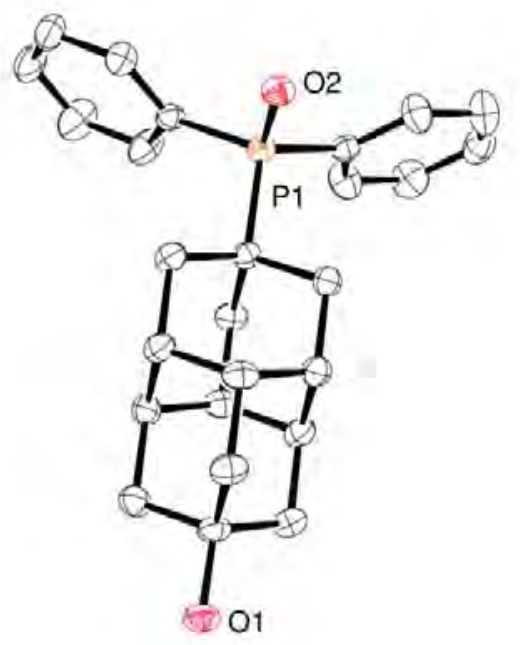

Ellipsoids are drawn at $50 \%$ probability.

Table S34. Crystal data and structure refinement for Compound 44 .

CCDC No.

Empirical formula

Formula weight

Temperature

Wavelength

Crystal system

Space group

Unit cell dimensions

Volume

Z

Density (calculated)

Absorption coefficient

$\mathrm{F}(000)$

Crystal size

Theta range for data collection

Index ranges

Reflections collected

Independent reflections

Completeness to theta $=25.242^{\circ}$

Absorption correction

Refinement method

Data / restraints / parameters
1053889

C26 H29 O2 P

404.46

$150(2) \mathrm{K}$

$0.71073 \AA$

Monoclinic

P 21/n

$\mathrm{a}=9.2389(18) \AA \quad \alpha=90^{\circ}$.

$\mathrm{b}=13.692(3) \AA \quad \beta=104.46(3)^{\circ}$.

$\mathrm{c}=16.878(3) \AA \quad \gamma=90^{\circ}$.

2067.3(8) $\AA^{3}$

4

$1.299 \mathrm{Mg} / \mathrm{m}^{3}$

$0.153 \mathrm{~mm}^{-1}$

864

$0.300 \times 0.200 \times 0.050 \mathrm{~mm}^{3}$

1.940 to $27.527^{\circ}$.

$-11<=\mathrm{h}<=11,-17<=\mathrm{k}<=17,-21<=1<=21$

46347

$4742[\mathrm{R}($ int $)=0.1347]$

$99.9 \%$

Empirical

Full-matrix least-squares on $\mathrm{F}^{2}$

4742 / 0 / 266 
Goodness-of-fit on $\mathrm{F}^{2}$

Final R indices [I $>2 \operatorname{sigma}(\mathrm{I})]$

$\mathrm{R}$ indices (all data)

Extinction coefficient

Largest diff. peak and hole
1.028

$\mathrm{R} 1=0.0461, \mathrm{wR} 2=0.1114$

$\mathrm{R} 1=0.0828, \mathrm{wR} 2=0.1252$

$\mathrm{n} / \mathrm{a}$

0.236 and -0.325 e. $\AA^{-3}$ 
Table S35. Bond lengths $[\AA]$ and angles $\left[{ }^{\circ}\right]$ for Compound 44.

\begin{tabular}{|c|c|}
\hline $\mathrm{P}(1)-\mathrm{O}(2)$ & $1.4963(15)$ \\
\hline$P(1)-C(21)$ & $1.808(2)$ \\
\hline $\mathrm{P}(1)-\mathrm{C}(15)$ & $1.814(2)$ \\
\hline $\mathrm{P}(1)-\mathrm{C}(8)$ & $1.832(2)$ \\
\hline $\mathrm{O}(1)-\mathrm{C}(12)$ & $1.437(2)$ \\
\hline $\mathrm{C}(2)-\mathrm{C}(1)$ & $1.535(3)$ \\
\hline$C(2)-C(11)$ & $1.538(3)$ \\
\hline $\mathrm{C}(2)-\mathrm{C}(3)$ & $1.542(3)$ \\
\hline $\mathrm{C}(3)-\mathrm{C}(9)$ & $1.534(3)$ \\
\hline$C(3)-C(4)$ & $1.536(3)$ \\
\hline$C(4)-C(5)$ & $1.537(3)$ \\
\hline $\mathrm{C}(4)-\mathrm{C}(13)$ & $1.539(3)$ \\
\hline$C(5)-C(10)$ & $1.536(3)$ \\
\hline$C(5)-C(6)$ & $1.546(3)$ \\
\hline$C(6)-C(14)$ & $1.531(3)$ \\
\hline $\mathrm{C}(6)-\mathrm{C}(1)$ & $1.533(3)$ \\
\hline$C(7)-C(1)$ & $1.537(3)$ \\
\hline$C(7)-C(8)$ & $1.547(3)$ \\
\hline $\mathrm{C}(8)-\mathrm{C}(9)$ & $1.542(3)$ \\
\hline $\mathrm{C}(8)-\mathrm{C}(10)$ & $1.544(3)$ \\
\hline $\mathrm{C}(11)-\mathrm{C}(12)$ & $1.531(3)$ \\
\hline$C(12)-C(13)$ & $1.526(3)$ \\
\hline$C(12)-C(14)$ & $1.528(3)$ \\
\hline$C(15)-C(20)$ & $1.385(3)$ \\
\hline$C(15)-C(16)$ & $1.396(3)$ \\
\hline$C(16)-C(17)$ & $1.381(3)$ \\
\hline$C(17)-C(18)$ & $1.388(4)$ \\
\hline $\mathrm{C}(18)-\mathrm{C}(19)$ & $1.383(4)$ \\
\hline$C(19)-C(20)$ & $1.384(3)$ \\
\hline$C(21)-C(22)$ & $1.393(3)$ \\
\hline$C(21)-C(26)$ & $1.399(3)$ \\
\hline $\mathrm{C}(22)-\mathrm{C}(23)$ & $1.386(3)$ \\
\hline $\mathrm{C}(23)-\mathrm{C}(24)$ & $1.383(4)$ \\
\hline$C(24)-C(25)$ & $1.376(3)$ \\
\hline$C(25)-C(26)$ & $1.395(3)$ \\
\hline $\mathrm{O}(2)-\mathrm{P}(1)-\mathrm{C}(21)$ & $109.59(9)$ \\
\hline $\mathrm{O}(2)-\mathrm{P}(1)-\mathrm{C}(15)$ & $110.04(9)$ \\
\hline
\end{tabular}




\begin{tabular}{|c|c|}
\hline $\mathrm{C}(21)-\mathrm{P}(1)-\mathrm{C}(15)$ & $110.27(10)$ \\
\hline $\mathrm{O}(2)-\mathrm{P}(1)-\mathrm{C}(8)$ & $110.45(9)$ \\
\hline $\mathrm{C}(21)-\mathrm{P}(1)-\mathrm{C}(8)$ & $108.39(9)$ \\
\hline $\mathrm{C}(15)-\mathrm{P}(1)-\mathrm{C}(8)$ & $108.06(9)$ \\
\hline$C(1)-C(2)-C(11)$ & $110.33(17)$ \\
\hline $\mathrm{C}(1)-\mathrm{C}(2)-\mathrm{C}(3)$ & $108.30(16)$ \\
\hline $\mathrm{C}(11)-\mathrm{C}(2)-\mathrm{C}(3)$ & $109.92(17)$ \\
\hline $\mathrm{C}(9)-\mathrm{C}(3)-\mathrm{C}(4)$ & $111.20(17)$ \\
\hline$C(9)-C(3)-C(2)$ & $110.19(17)$ \\
\hline $\mathrm{C}(4)-\mathrm{C}(3)-\mathrm{C}(2)$ & $108.45(16)$ \\
\hline$C(3)-C(4)-C(5)$ & $108.36(16)$ \\
\hline$C(3)-C(4)-C(13)$ & $110.93(18)$ \\
\hline$C(5)-C(4)-C(13)$ & $110.97(17)$ \\
\hline$C(10)-C(5)-C(4)$ & $111.15(17)$ \\
\hline $\mathrm{C}(10)-\mathrm{C}(5)-\mathrm{C}(6)$ & $110.91(17)$ \\
\hline$C(4)-C(5)-C(6)$ & $107.76(17)$ \\
\hline$C(14)-C(6)-C(1)$ & $110.21(17)$ \\
\hline$C(14)-C(6)-C(5)$ & $110.78(17)$ \\
\hline$C(1)-C(6)-C(5)$ & $108.24(16)$ \\
\hline$C(1)-C(7)-C(8)$ & $109.70(16)$ \\
\hline$C(9)-C(8)-C(10)$ & $108.67(17)$ \\
\hline $\mathrm{C}(9)-\mathrm{C}(8)-\mathrm{C}(7)$ & $109.04(17)$ \\
\hline $\mathrm{C}(10)-\mathrm{C}(8)-\mathrm{C}(7)$ & $108.74(16)$ \\
\hline $\mathrm{C}(9)-\mathrm{C}(8)-\mathrm{P}(1)$ & $107.74(13)$ \\
\hline $\mathrm{C}(10)-\mathrm{C}(8)-\mathrm{P}(1)$ & $115.54(14)$ \\
\hline$C(7)-C(8)-P(1)$ & $106.96(13)$ \\
\hline $\mathrm{C}(3)-\mathrm{C}(9)-\mathrm{C}(8)$ & $109.84(16)$ \\
\hline$C(5)-C(10)-C(8)$ & $109.64(17)$ \\
\hline$C(12)-C(11)-C(2)$ & $109.76(17)$ \\
\hline $\mathrm{O}(1)-\mathrm{C}(12)-\mathrm{C}(13)$ & $107.94(16)$ \\
\hline $\mathrm{O}(1)-\mathrm{C}(12)-\mathrm{C}(14)$ & $110.86(16)$ \\
\hline$C(13)-C(12)-C(14)$ & $108.96(18)$ \\
\hline $\mathrm{O}(1)-\mathrm{C}(12)-\mathrm{C}(11)$ & $110.02(17)$ \\
\hline$C(13)-C(12)-C(11)$ & $109.43(17)$ \\
\hline$C(14)-C(12)-C(11)$ & $109.59(17)$ \\
\hline$C(12)-C(13)-C(4)$ & $109.29(17)$ \\
\hline$C(12)-C(14)-C(6)$ & $109.94(16)$ \\
\hline$C(20)-C(15)-C(16)$ & $118.1(2)$ \\
\hline$C(20)-C(15)-P(1)$ & $115.68(17)$ \\
\hline$C(16)-C(15)-P(1)$ & $126.13(16)$ \\
\hline$C(17)-C(16)-C(15)$ & $121.1(2)$ \\
\hline
\end{tabular}




$\begin{array}{ll}\mathrm{C}(16)-\mathrm{C}(17)-\mathrm{C}(18) & 120.0(2) \\ \mathrm{C}(19)-\mathrm{C}(18)-\mathrm{C}(17) & 119.6(2) \\ \mathrm{C}(18)-\mathrm{C}(19)-\mathrm{C}(20) & 120.1(2) \\ \mathrm{C}(19)-\mathrm{C}(20)-\mathrm{C}(15) & 121.2(2) \\ \mathrm{C}(22)-\mathrm{C}(21)-\mathrm{C}(26) & 118.42(19) \\ \mathrm{C}(22)-\mathrm{C}(21)-\mathrm{P}(1) & 125.85(16) \\ \mathrm{C}(26)-\mathrm{C}(21)-\mathrm{P}(1) & 115.70(16) \\ \mathrm{C}(23)-\mathrm{C}(22)-\mathrm{C}(21) & 120.5(2) \\ \mathrm{C}(24)-\mathrm{C}(23)-\mathrm{C}(22) & 120.5(2) \\ \mathrm{C}(25)-\mathrm{C}(24)-\mathrm{C}(23) & 120.0(2) \\ \mathrm{C}(24)-\mathrm{C}(25)-\mathrm{C}(26) & 119.9(2) \\ \mathrm{C}(25)-\mathrm{C}(26)-\mathrm{C}(21) & 120.7(2) \\ \mathrm{C}(6)-\mathrm{C}(1)-\mathrm{C}(2) & 109.12(16) \\ \mathrm{C}(6)-\mathrm{C}(1)-\mathrm{C}(7) & 110.62(17) \\ \mathrm{C}(2)-\mathrm{C}(1)-\mathrm{C}(7) & 110.38(17) \\ \end{array}$




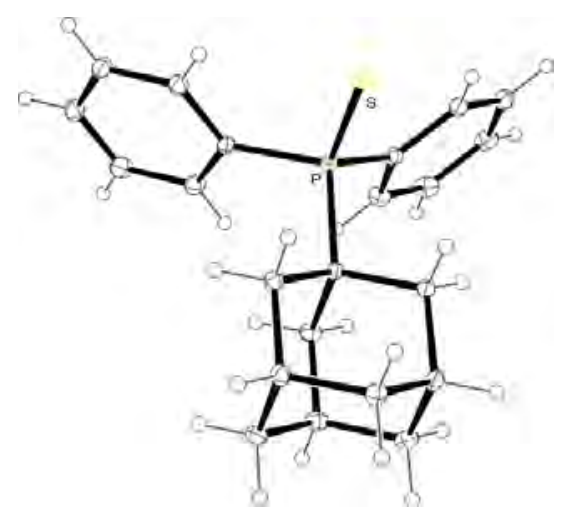

Ellipsoids are drawn at $50 \%$ probability.

Table S36. Crystal data and structure refinement for Compound 46.

$\mathrm{N}^{\circ} \mathrm{CCDC}$

Empirical formula

Formula weight

Temperature

Wavelength

Crystal system

Space group

Unit cell dimensions

Volume

Z

Density (calculated)

Absorption coefficient

$\mathrm{F}(000)$

Crystal size

Theta range for data collection

Index ranges

Reflections collected

Independent reflections

Completeness to theta $=27.48^{\circ}$

Max. and min. transmission

Refinement method

Data / restraints / parameters

Goodness-of-fit on $\mathrm{F}^{2}$

Final $\mathrm{R}$ indices $[\mathrm{I}>2 \operatorname{sigma}(\mathrm{I})]$

$\mathrm{R}$ indices (all data)

Largest diff. peak and hole
1054568

$\mathrm{C}_{22} \mathrm{H}_{25} \mathrm{PS}$

352.45

115(2) K

$0.71073 \AA$

Orthorhombic

P n m a

$\mathrm{a}=11.2136(3) \AA$ $\alpha=90^{\circ}$.

$\mathrm{b}=12.6017(4) \AA$ $\beta=90^{\circ}$.

$\mathrm{c}=13.2583(5) \AA$ $\gamma=90^{\circ}$.

1873.54(11) $\AA^{3}$

4

$1.250 \mathrm{Mg} / \mathrm{m}^{3}$

$0.258 \mathrm{~mm}^{-1}$

752

$0.25 \times 0.20 \times 0.15 \mathrm{~mm}^{3}$

3.57 to $27.48^{\circ}$.

$-11<=\mathrm{h}<=14,-16<=\mathrm{k}<=16,-17<=\mathrm{l}<=17$

6866

$2231[\mathrm{R}($ int $)=0.0321]$

$99.3 \%$

0.9623 and 0.9382

Full-matrix least-squares on $\mathrm{F}^{2}$

$2231 / 0 / 118$

1.075

$\mathrm{R} 1=0.0357, \mathrm{wR} 2=0.0770$

$\mathrm{R} 1=0.0484, \mathrm{wR} 2=0.0840$

0.399 and -0.257 e. $\AA^{-3}$ 
Table S37. Atomic coordinates ( $\left.\times 10^{4}\right)$ and equivalent isotropic displacement parameters $\left(\AA^{2} \times 10^{3}\right)$ for Compound 46. U(eq) is defined as one third of the trace of the orthogonalized $\mathrm{U}^{\mathrm{ij}}$ tensor.

\begin{tabular}{lrlcc}
\hline & $\mathrm{x}$ & $\mathrm{y}$ & $\mathrm{z}$ & $\mathrm{U}(\mathrm{eq})$ \\
\hline $\mathrm{C}(1)$ & & & & $14(1)$ \\
$\mathrm{C}(2)$ & $406(2)$ & 2500 & $5586(2)$ & $19(1)$ \\
$\mathrm{C}(3)$ & $-795(2)$ & 2500 & $6150(2)$ & $21(1)$ \\
$\mathrm{C}(4)$ & $-1825(2)$ & 2500 & $5378(2)$ & $22(1)$ \\
$\mathrm{C}(5)$ & $-1745(1)$ & $1506(1)$ & $4715(1)$ & $18(1)$ \\
$\mathrm{C}(6)$ & $-553(1)$ & $1506(1)$ & $4146(1)$ & $17(1)$ \\
$\mathrm{C}(8)$ & $477(1)$ & $1500(1)$ & $4916(1)$ & $19(1)$ \\
$\mathrm{C}(9)$ & $-467(2)$ & 2500 & $3485(2)$ & $16(1)$ \\
$\mathrm{C}(10)$ & $1644(1)$ & $3676(1)$ & $7243(1)$ & $22(1)$ \\
$\mathrm{C}(11)$ & $751(1)$ & $3834(1)$ & $7964(1)$ & $26(1)$ \\
$\mathrm{C}(12)$ & $757(2)$ & $4745(2)$ & $8562(1)$ & $24(1)$ \\
$\mathrm{C}(13)$ & $1647(2)$ & $5499(1)$ & $8456(1)$ & $19(1)$ \\
$\mathrm{C}(14)$ & $2531(2)$ & $5346(1)$ & $7740(1)$ & $14(1)$ \\
$\mathrm{P}$ & $2527(1)$ & $4448(1)$ & $7136(1)$ & $18(1)$ \\
$\mathrm{S}$ & $1727(1)$ & 2500 & $6433(1)$ & \\
\hline
\end{tabular}


Table S38. Bond lengths $[\AA]$ and angles $\left[^{\circ}\right]$ for Compound 46.

\begin{tabular}{|c|c|}
\hline $\mathrm{C}(1)-\mathrm{C}(2)$ & $1.540(3)$ \\
\hline$C(1)-C(6)$ & $1.5435(18)$ \\
\hline $\mathrm{C}(1)-\mathrm{C}(6) \# 1$ & $1.5435(18)$ \\
\hline $\mathrm{C}(1)-\mathrm{P}$ & $1.859(2)$ \\
\hline $\mathrm{C}(2)-\mathrm{C}(3)$ & $1.544(3)$ \\
\hline $\mathrm{C}(2)-\mathrm{H}(2 \mathrm{~A})$ & 0.9900 \\
\hline $\mathrm{C}(2)-\mathrm{H}(2 \mathrm{~B})$ & 0.9900 \\
\hline$C(3)-C(4)$ & $1.532(2)$ \\
\hline $\mathrm{C}(3)-\mathrm{C}(4) \# 1$ & $1.532(2)$ \\
\hline $\mathrm{C}(3)-\mathrm{H}(3)$ & 1.0000 \\
\hline$C(4)-C(5)$ & $1.534(2)$ \\
\hline $\mathrm{C}(4)-\mathrm{H}(4 \mathrm{~A})$ & 0.9900 \\
\hline $\mathrm{C}(4)-\mathrm{H}(4 \mathrm{~B})$ & 0.9900 \\
\hline$C(5)-C(8)$ & $1.5317(19)$ \\
\hline$C(5)-C(6)$ & $1.542(2)$ \\
\hline $\mathrm{C}(5)-\mathrm{H}(5)$ & 1.0000 \\
\hline $\mathrm{C}(6)-\mathrm{H}(6 \mathrm{~A})$ & 0.9900 \\
\hline $\mathrm{C}(6)-\mathrm{H}(6 \mathrm{~B})$ & 0.9900 \\
\hline $\mathrm{C}(8)-\mathrm{C}(5) \# 1$ & $1.5317(19)$ \\
\hline $\mathrm{C}(8)-\mathrm{H}(8 \mathrm{~A})$ & 0.9900 \\
\hline $\mathrm{C}(8)-\mathrm{H}(8 \mathrm{~B})$ & 0.9900 \\
\hline C(9)-C(14) & $1.395(2)$ \\
\hline C(9)-C(10) & $1.399(2)$ \\
\hline $\mathrm{C}(9)-\mathrm{P}$ & $1.8322(15)$ \\
\hline $\mathrm{C}(10)-\mathrm{C}(11)$ & $1.394(2)$ \\
\hline $\mathrm{C}(10)-\mathrm{H}(10)$ & 0.9500 \\
\hline $\mathrm{C}(11)-\mathrm{C}(12)$ & $1.386(2)$ \\
\hline $\mathrm{C}(11)-\mathrm{H}(11)$ & 0.9500 \\
\hline$C(12)-C(13)$ & $1.386(2)$ \\
\hline $\mathrm{C}(12)-\mathrm{H}(12)$ & 0.9500 \\
\hline $\mathrm{C}(13)-\mathrm{C}(14)$ & $1.387(2)$ \\
\hline $\mathrm{C}(13)-\mathrm{H}(13)$ & 0.9500 \\
\hline $\mathrm{C}(14)-\mathrm{H}(14)$ & 0.9500 \\
\hline $\mathrm{P}-\mathrm{C}(9) \# 1$ & $1.8322(15)$ \\
\hline P-S & $1.9717(7)$ \\
\hline$C(2)-C(1)-C(6)$ & $108.94(11)$ \\
\hline $\mathrm{C}(2)-\mathrm{C}(1)-\mathrm{C}(6) \# 1$ & $108.94(11)$ \\
\hline$C(6)-C(1)-C(6) \# 1$ & $109.42(17)$ \\
\hline$C(2)-C(1)-P$ & $113.80(14)$ \\
\hline$C(6)-C(1)-P$ & $107.83(10)$ \\
\hline$C(6) \# 1-C(1)-P$ & $107.83(10)$ \\
\hline$C(1)-C(2)-C(3)$ & $109.42(17)$ \\
\hline $\mathrm{C}(1)-\mathrm{C}(2)-\mathrm{H}(2 \mathrm{~A})$ & 109.8 \\
\hline $\mathrm{C}(3)-\mathrm{C}(2)-\mathrm{H}(2 \mathrm{~A})$ & 109.8 \\
\hline $\mathrm{C}(1)-\mathrm{C}(2)-\mathrm{H}(2 \mathrm{~B})$ & 109.8 \\
\hline $\mathrm{C}(3)-\mathrm{C}(2)-\mathrm{H}(2 \mathrm{~B})$ & 109.8 \\
\hline $\mathrm{H}(2 \mathrm{~A})-\mathrm{C}(2)-\mathrm{H}(2 \mathrm{~B})$ & 108.2 \\
\hline$C(4)-C(3)-C(4) \# 1$ & $109.59(18)$ \\
\hline $\mathrm{C}(4)-\mathrm{C}(3)-\mathrm{C}(2)$ & $109.64(12)$ \\
\hline $\mathrm{C}(4) \# 1-\mathrm{C}(3)-\mathrm{C}(2)$ & $109.64(12)$ \\
\hline $\mathrm{C}(4)-\mathrm{C}(3)-\mathrm{H}(3)$ & 109.3 \\
\hline $\mathrm{C}(4) \# 1-\mathrm{C}(3)-\mathrm{H}(3)$ & 109.3 \\
\hline $\mathrm{C}(2)-\mathrm{C}(3)-\mathrm{H}(3)$ & 109.3 \\
\hline$C(3)-C(4)-C(5)$ & $109.50(14)$ \\
\hline $\mathrm{C}(3)-\mathrm{C}(4)-\mathrm{H}(4 \mathrm{~A})$ & 109.8 \\
\hline $\mathrm{C}(5)-\mathrm{C}(4)-\mathrm{H}(4 \mathrm{~A})$ & 109.8 \\
\hline $\mathrm{C}(3)-\mathrm{C}(4)-\mathrm{H}(4 \mathrm{~B})$ & 109.8 \\
\hline $\mathrm{C}(5)-\mathrm{C}(4)-\mathrm{H}(4 \mathrm{~B})$ & 109.8 \\
\hline $\mathrm{H}(4 \mathrm{~A})-\mathrm{C}(4)-\mathrm{H}(4 \mathrm{~B})$ & 108.2 \\
\hline $\mathrm{C}(8)-\mathrm{C}(5)-\mathrm{C}(4)$ & $109.62(14)$ \\
\hline $\mathrm{C}(8)-\mathrm{C}(5)-\mathrm{C}(6)$ & $109.58(14)$ \\
\hline
\end{tabular}




\begin{tabular}{|c|c|}
\hline $\mathrm{C}(4)-\mathrm{C}(5)-\mathrm{C}(6)$ & $109.06(12)$ \\
\hline $\mathrm{C}(8)-\mathrm{C}(5)-\mathrm{H}(5)$ & 109.5 \\
\hline $\mathrm{C}(4)-\mathrm{C}(5)-\mathrm{H}(5)$ & 109.5 \\
\hline $\mathrm{C}(6)-\mathrm{C}(5)-\mathrm{H}(5)$ & 109.5 \\
\hline$C(5)-C(6)-C(1)$ & $109.79(13)$ \\
\hline $\mathrm{C}(5)-\mathrm{C}(6)-\mathrm{H}(6 \mathrm{~A})$ & 109.7 \\
\hline $\mathrm{C}(1)-\mathrm{C}(6)-\mathrm{H}(6 \mathrm{~A})$ & 109.7 \\
\hline $\mathrm{C}(5)-\mathrm{C}(6)-\mathrm{H}(6 \mathrm{~B})$ & 109.7 \\
\hline $\mathrm{C}(1)-\mathrm{C}(6)-\mathrm{H}(6 \mathrm{~B})$ & 109.7 \\
\hline $\mathrm{H}(6 \mathrm{~A})-\mathrm{C}(6)-\mathrm{H}(6 \mathrm{~B})$ & 108.2 \\
\hline $\mathrm{C}(5)-\mathrm{C}(8)-\mathrm{C}(5) \# 1$ & $109.77(17)$ \\
\hline $\mathrm{C}(5)-\mathrm{C}(8)-\mathrm{H}(8 \mathrm{~A})$ & 109.7 \\
\hline $\mathrm{C}(5) \# 1-\mathrm{C}(8)-\mathrm{H}(8 \mathrm{~A})$ & 109.7 \\
\hline $\mathrm{C}(5)-\mathrm{C}(8)-\mathrm{H}(8 \mathrm{~B})$ & 109.7 \\
\hline $\mathrm{C}(5) \# 1-\mathrm{C}(8)-\mathrm{H}(8 \mathrm{~B})$ & 109.7 \\
\hline $\mathrm{H}(8 \mathrm{~A})-\mathrm{C}(8)-\mathrm{H}(8 \mathrm{~B})$ & 108.2 \\
\hline $\mathrm{C}(14)-\mathrm{C}(9)-\mathrm{C}(10)$ & $118.53(14)$ \\
\hline $\mathrm{C}(14)-\mathrm{C}(9)-\mathrm{P}$ & $117.94(11)$ \\
\hline$C(10)-C(9)-P$ & $123.53(12)$ \\
\hline $\mathrm{C}(11)-\mathrm{C}(10)-\mathrm{C}(9)$ & $120.17(15)$ \\
\hline $\mathrm{C}(11)-\mathrm{C}(10)-\mathrm{H}(10)$ & 119.9 \\
\hline $\mathrm{C}(9)-\mathrm{C}(10)-\mathrm{H}(10)$ & 119.9 \\
\hline$C(12)-C(11)-C(10)$ & $120.67(15)$ \\
\hline $\mathrm{C}(12)-\mathrm{C}(11)-\mathrm{H}(11)$ & 119.7 \\
\hline $\mathrm{C}(10)-\mathrm{C}(11)-\mathrm{H}(11)$ & 119.7 \\
\hline$C(11)-C(12)-C(13)$ & $119.34(15)$ \\
\hline $\mathrm{C}(11)-\mathrm{C}(12)-\mathrm{H}(12)$ & 120.3 \\
\hline $\mathrm{C}(13)-\mathrm{C}(12)-\mathrm{H}(12)$ & 120.3 \\
\hline $\mathrm{C}(12)-\mathrm{C}(13)-\mathrm{C}(14)$ & $120.37(15)$ \\
\hline $\mathrm{C}(12)-\mathrm{C}(13)-\mathrm{H}(13)$ & 119.8 \\
\hline $\mathrm{C}(14)-\mathrm{C}(13)-\mathrm{H}(13)$ & 119.8 \\
\hline $\mathrm{C}(13)-\mathrm{C}(14)-\mathrm{C}(9)$ & $120.92(14)$ \\
\hline $\mathrm{C}(13)-\mathrm{C}(14)-\mathrm{H}(14)$ & 119.5 \\
\hline $\mathrm{C}(9)-\mathrm{C}(14)-\mathrm{H}(14)$ & 119.5 \\
\hline C(9)\#1-P-C(9) & $107.94(9)$ \\
\hline C(9)\#1-P-C(1) & $108.26(6)$ \\
\hline $\mathrm{C}(9)-\mathrm{P}-\mathrm{C}(1)$ & $108.26(6)$ \\
\hline C(9)\#1-P-S & $110.65(5)$ \\
\hline C(9)-P-S & $110.65(5)$ \\
\hline C(1)-P-S & $110.98(7)$ \\
\hline
\end{tabular}

Symmetry transformations used to generate equivalent atoms:

\#1 x,-y+1/2,z 
Table S39. Anisotropic displacement parameters $\left(\AA^{2} \times 10^{3}\right)$ for Compound 46. The anisotropic displacement factor exponent takes the form: $-2 \pi^{2}\left[h^{2} a^{* 2} U^{11}+\ldots+2 h k a^{*} b^{*} U^{12}\right]$

\begin{tabular}{lcccccc}
\hline & $\mathrm{U}^{11}$ & $\mathrm{U}^{22}$ & $\mathrm{U}^{33}$ & $\mathrm{U}^{23}$ & $\mathrm{U}^{13}$ & $\mathrm{U}^{12}$ \\
\hline $\mathrm{C}(1)$ & $15(1)$ & $13(1)$ & $13(1)$ & 0 & $-1(1)$ & 0 \\
$\mathrm{C}(2)$ & $15(1)$ & $25(1)$ & $15(1)$ & 0 & $1(1)$ & 0 \\
$\mathrm{C}(3)$ & $15(1)$ & $29(1)$ & $18(1)$ & 0 & $1(1)$ & 0 \\
$\mathrm{C}(4)$ & $20(1)$ & $24(1)$ & $22(1)$ & $2(1)$ & $-4(1)$ & $-5(1)$ \\
$\mathrm{C}(5)$ & $23(1)$ & $15(1)$ & $18(1)$ & $-2(1)$ & $-3(1)$ & $-1(1)$ \\
$\mathrm{C}(6)$ & $19(1)$ & $15(1)$ & $18(1)$ & $-1(1)$ & $-2(1)$ & $1(1)$ \\
$\mathrm{C}(8)$ & $22(1)$ & $20(1)$ & $16(1)$ & 0 & $-1(1)$ & 0 \\
$\mathrm{C}(9)$ & $18(1)$ & $16(1)$ & $15(1)$ & $0(1)$ & $-4(1)$ & $1(1)$ \\
$\mathrm{C}(10)$ & $21(1)$ & $25(1)$ & $19(1)$ & $-4(1)$ & $0(1)$ & $-1(1)$ \\
$\mathrm{C}(11)$ & $25(1)$ & $33(1)$ & $21(1)$ & $-8(1)$ & $-2(1)$ & $4(1)$ \\
$\mathrm{C}(12)$ & $31(1)$ & $23(1)$ & $25(1)$ & $-9(1)$ & $-10(1)$ & $5(1)$ \\
$\mathrm{C}(13)$ & $26(1)$ & $17(1)$ & $29(1)$ & $-1(1)$ & $-9(1)$ & $-2(1)$ \\
$\mathrm{C}(14)$ & $21(1)$ & $18(1)$ & $20(1)$ & $1(1)$ & $-2(1)$ & $1(1)$ \\
$\mathrm{P}$ & $14(1)$ & $14(1)$ & $13(1)$ & 0 & $0(1)$ & 0 \\
$\mathrm{~S}$ & $15(1)$ & $21(1)$ & $17(1)$ & 0 & $2(1)$ & 0 \\
& & & & & & \\
\hline
\end{tabular}

(1) Grimme, S.; Ehrlich, S.; Goerigk, L. J. Comput. Chem. 2011, 32, 1456.

(2) Baechler, R. D.; Mislow, K. J. Am. Chem. Soc. 1970, 92, 3090. 